\title{
Investigation of Transition Scenarios in Boundary-Layer Flows
}

\author{
Dissertation \\ zur Erlangung des Doktorgrades \\ der Mathematisch-Naturwissenschaftlichen Fakultäten \\ der Georg-August-Universität zu Göttingen
}

vorgelegt von

Andreas Stolte

aus Harderberg

Göttingen 1999 


\section{7}

Referent:

Korreferent:

Tag der mündlichen Prüfung: 4. November 1999
Prof. Dr. H. Eckelmann

Prof. Dr. D. Ronneberger 


\section{Contents}

List of Symbols $\quad$ v

1 Introduction 1

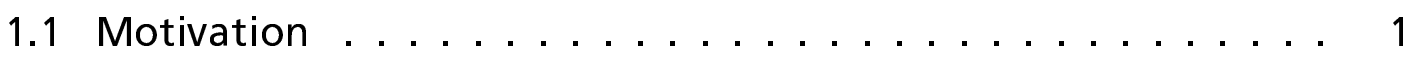

1.2 Previous Research . . . . . . . . . . . . . . . . . 2 2

1.3 Goals of this Work . . . . . . . . . . . . . . . . . . . . . . 5

2 The DLR Swept Plate Experiment 7

2.1 Experimental Setup . . . . . . . . . . . . . . 7

2.2 Definition of a Model Flow . . . . . . . . . . . . . . 8

2.3 The Laminar Base Flow . . . . . . . . . . . . . . . . . . . . 10

3 Linear Nonlocal Instability Analysis $\quad 15$

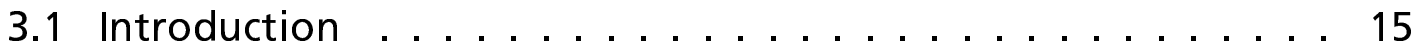

3.2 The Parabolized Stability Equations . . . . . . . . . . . . . . . 16

3.3 Numerical Aspects . . . . . . . . . . . . . . . . . . . . . . 19

3.4 Initial Conditions . . . . . . . . . . . . . . . . . . 21

3.5 Parametric Investigations . . . . . . . . . . . . . . 27

4 Nonlinear Instability Analysis and Disturbance Interaction 33

4.1 Theoretical Background . . . . . . . . . . . . . . . . . . . . 34

4.2 Nonlinear Growth in a Parallel Base Flow . . . . . . . . . . . . 37

4.3 Nonlinear Amplification of Cross-Flow Vortices . . . . . . . . . . 40

4.4 Wave-Vortex Interaction . . . . . . . . . . . . . . 51

4.5 The "Saturated" State . . . . . . . . . . . . . . . . . . 60 
5 Secondary Instability Analysis and Laminar-Turbulent Transition 71

5.1 Introduction . . . . . . . . . . . . . . 71

5.2 Secondary Instability Theory . . . . . . . . . . . . . . . 72

5.3 Numerical Solution Procedure . . . . . . . . . . . . . . 76

5.4 High Frequency Secondary Instability . . . . . . . . . . 77

5.4.1 Limit of Low Primary Vortex Amplitude . . . . . . . . 78

5.4.2 Propagation of Secondary Disturbances . . . . . . . 82

5.4.3 Onset of High Frequency Secondary Instability . . . . . 83

5.4.4 Secondary Instability of a Strongly Distorted Base Flow . 97

6 Concluding Remarks $\quad 117$

6.1 Summary . . . . . . . . . . . . . . . . . . . 117

6.2 Outlook . . . . . . . . . . . . . . . . . . 119

$\begin{array}{ll}\text { Acknowledgements } & 129\end{array}$ 


\section{List of Symbols}

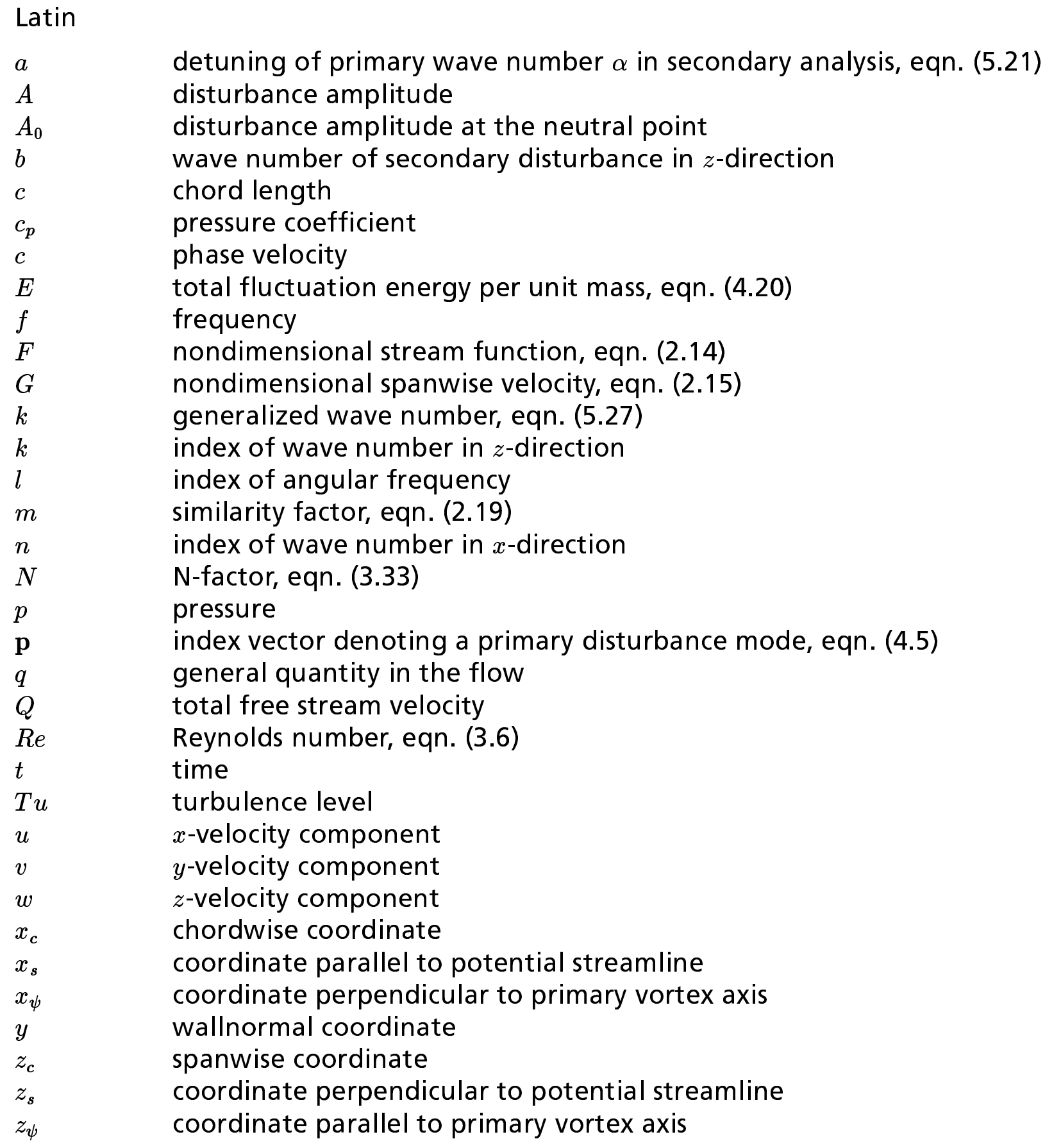

$a$

A

$A_{0}$

$b$

$c$

$c_{p}$

$c$

E

$f$

F

G

$k$

$k$

$l$

$m$

$n$

$N$

$p$

p

$q$

$Q$

$R e$

$t$

Tu

$u$

$v$

$w$

$x_{c}$

$x_{s}$

$x_{\psi}$

$y$

$z_{c}$

$z_{s}$

$z_{\psi}$

detuning of primary wave number $\alpha$ in secondary analysis, eqn. (5.21) disturbance amplitude disturbance amplitude at the neutral point wave number of secondary disturbance in $z$-direction chord length pressure coefficient phase velocity total fluctuation energy per unit mass, eqn. (4.20) frequency nondimensional stream function, eqn. (2.14) nondimensional spanwise velocity, eqn. (2.15) generalized wave number, eqn. (5.27) index of wave number in $z$-direction index of angular frequency similarity factor, eqn. (2.19) index of wave number in $x$-direction $\mathrm{N}$-factor, eqn. (3.33) pressure index vector denoting a primary disturbance mode, eqn. (4.5) general quantity in the flow total free stream velocity Reynolds number, eqn. (3.6)

time

turbulence level $x$-velocity component $y$-velocity component $z$-velocity component chordwise coordinate coordinate parallel to potential streamline coordinate perpendicular to primary vortex axis wallnormal coordinate spanwise coordinate coordinate perpendicular to potential streamline coordinate parallel to primary vortex axis 


\section{Greek}

$\begin{array}{ll}\alpha & \text { wave number of primary disturbance in } x \text {-direction } \\ \beta & \text { wave number of primary disturbance in } z \text {-direction } \\ \gamma & \text { growth rate of primary disturbance in } x \text {-direction } \\ \delta & \text { detuning parameter, eqn. (5.26) } \\ \zeta & z \text {-vorticity component } \\ \eta & y \text {-vorticity component } \\ \theta & \text { phase function, eqn. (3.17) } \\ \lambda & \text { wave length } \\ \mu & \text { dynamic viscosity } \\ \nu & \text { kinematic viscosity } \\ \xi & \text { x-vorticity component } \\ \rho & \text { density } \\ \sigma & \text { weight in generalized auxiliary condition, eqn. (4.10) } \\ \sigma & \text { temporal growth rate and frequency shift of secondary disturbance } \\ \phi & \text { orientation of potential streamline w.r.t. } x_{c} \text {-axis } \\ \phi & \text { effective sweep angle } \\ \chi & \text { local similarity variable, eqn. (2.13) } \\ \chi & \text { wave function, eqn. (3.16) } \\ \psi & \text { orientation of primary disturbance w.r.t. } x_{c} \text {-axis, eqn. (3.31) } \\ \Psi & \text { stream function, eqn.(2.10) } \\ \omega & \text { angular frequency }\end{array}$

Superscripts and marks

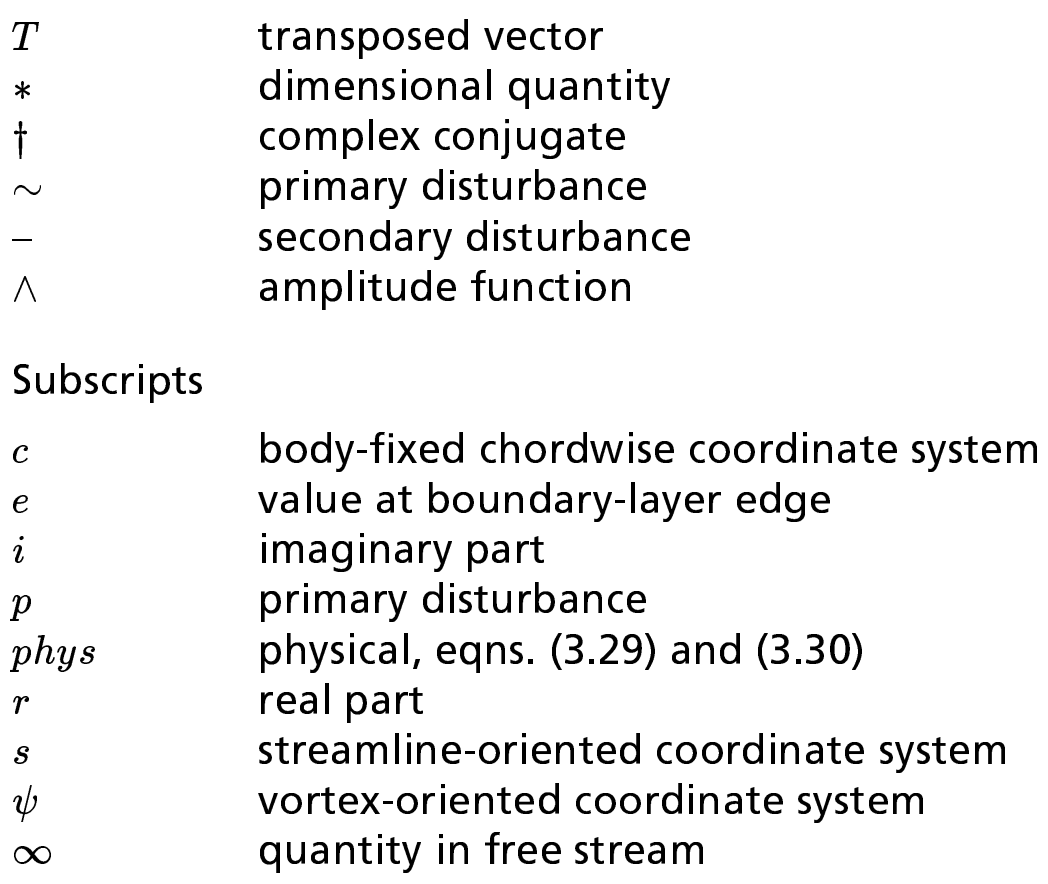




\section{Chapter 1}

\section{Introduction}

\subsection{Motivation}

The transition of regular, laminar flow into irregular, turbulent fluid motion is a fascinating process of fundamental importance in fluid mechanics. Because of the significant effect of transition in shear flows on skin friction, heat transfer and flow separation, the prediction and control of laminar-turbulent transition is also a central task in a great variety of engineering applications. Therefore, it is not astonishing that, for a long time, researchers, driven by both scientific and technological interest, are striving hard for insight into the mechanisms associated with the transition process. After more than a century of intensive research, answers to many questions have been found, but a general understanding of laminar-turbulent transition is still missing. On account of the extreme difficulty of the transition problem, two-dimensional flows have chiefly been investigated in the past, even though many flows in nature and in technological applications clearly are three-dimensional.

Three-dimensionality is an essential attribute of boundary-layer flows over wings and fins of commercial airplanes, since these components are swept in order to avoid shocks at higher cruising speed. In such boundary layers, a certain type of instability, called crossflow instability, leads to strong amplification of disturbances in the region of accelerated flow and, hence, plays a key role in the transition process. The improvement of methods for transition prediction and control requires a profound understanding of disturbance generation, i.e. receptivity, disturbance amplification, and of the later stages of transition leading to turbulent breakdown. The present work focuses on the mechanisms relevant to the nonlinear later stages of transition scenarios dominated by crossflow instability. 


\subsection{Previous Research}

The analytical study of the relationship between instability and transition starts in the last third of the 19th century with the work of Helmholtz [37], Lord Kelvin [49], and Lord Rayleigh [77], who studied the inertial instability of inviscid, incompressible flows. The theoretical work of Lord Rayleigh is based on the conception, that laminar flow is unstable with respect to small disturbances, which are amplified and finally lead the flow into turbulence. He showed that the presence of an inflection point in the local velocity profile is necessary for the occurrence of unstable waves in inviscid flows. In 1883, Reynolds [81] performed his famous pipe experiments. He studied the variation of the flow rate of fluids through transparent circular pipes of varying sizes at different velocities and observed both smooth and sinuous or turbulent motion of injected streams of dye. By using dimensional analysis, he found that these different types of motion can be characterized by one dimensionless parameter, the Reynolds number.

However, the eigenvalues of the linearized equations - these are all stable for the pipe flow - do not completely describe amplification and damping of small disturbances. As a consequence of the non-normality of the linearized equations of motion, the corresponding eigenvectors are not orthogonal. Therefore, small disturbances can grow transitorily even in the case of global stability. Nonlinear terms can then redistribute the disturbance energy. This mechanism of transient growth has been studied in detail during the last decades (see e. g. Großmann [30]), but it is not considered here.

After Prandtl [76] introduced the concept of the boundary layer in 1904, Orr [71] and Sommerfeld [90] independently derived an equation capturing the destabilizing effect of viscosity in two-dimensional flows. Considering plane Poiseuille flow, Heisenberg [36] showed that inviscidly stable flows can be unstable for finite Reynolds numbers. The Orr-Sommerfeld equation was solved by Tollmien [93] for the incompressible flow over a flat plate, the Blasius boundary-layer flow. Tollmien calculated a curve enclosing the region of instability and a critical value for the Reynolds number, below which the laminar flow is stable. Schlichting [83] computed growth rates of unstable disturbance waves. The first experimental verification of the existence of such waves by Schubauer \& Skramstad [86] led to the acceptance of the relevance of linear instability theory to transition in the scientific community. The first transition criterion for practical applications was developed by Liepmann [58] from linear instability results. Such criteria, correlating the transition location with a certain degree of disturbance amplification, are still widely used for transition prediction in aerodynamic design. An extensive survey of the possibilities of transition prediction by using the eigenvalues of the classical linear stability equations is given by Arnal [2]. Gaster [26] examined the relationship between temporally and and spatially grow- 
ing disturbances and gave a relationship between these growth concepts. A detailed summary of classical linear instability theory for boundary layers is given by Mack [59].

The effect of base flow nonparallelism on the growth rates of twodimensional waves has been examined by Bouthier [14] and Gaster [27]. While Bouthier found significant destabilizing influence of nonparallelism, Gaster observed only a small effect on the curve of neutral amplification. $\mathrm{His}$ results have been confirmed by Herbert and Bertolotti [41]. Bertolotti [6] discusses these studies and shows that the effect of nonparallelism is strongly dependent on the definition of disturbance growth. Using the parabolized stability equations (PSE), which exploit the convective nature of the disturbances to convert the elliptic disturbance equations into a system of parabolic differential equations, he also found rather small influence of nonparallelism on neutral curves and growth rates of two-dimensional waves. Independently of the development of the PSE-method by Herbert and Bertolotti (cf. Bertolotti [6]), Dallmann and Simen (cf. Simen [87]) developed a nonlocal instability theory, which leads to equations identical to the PSE. One important strength of these methods is their ability to incorporate nonlinear effects. In the few years since the derivation of the PSE, this method has already been applied by a number of researchers to a large variety of flows (see, for example, Chang et al. [16], Hein et al. [33], Wang et al. [95], Malik et al. [61], Airiau \& Casalis [1], Schrauf et al. [85], Haynes \& Reed [32], Janke \& Balakumar [45]). The review by Herbert [40] discusses in detail the PSE-method and its applications.

That transition on a swept wing occurs much further upstream than on a corresponding unswept wing, was first observed by Gray [28], who evaluated flight tests at the British Royal Aircraft Establishment. Flow visualizations displayed stationary, regularly spaced rows in the streamwise direction prior to transition. Such patterns are generated by the action of stationary vortices. The instability leading to the amplification of those vortices is caused by inflection points in crossflow profiles of the boundary layer (cf. Owen \& Randall [73]). For the model problem of a rotating disk, Gregory et al. [29], performed experimental and theoretical studies and found, that both traveling waves and stationary vortices, approximately aligned with the potential streamline, can be amplified in three-dimensional boundary-layer flow.

Experimental studies of instability and transition in the three-dimensional boundary layer on a swept cylinder have been performed by Poll [75], who investigated the attachment-line instability near the leading edge, the effect of convex surface curvature, and the dependence of disturbance growth on the sweep angle. Arnal et al. [3] considered the flow on a swept wing, Saric \& Yeates [82] investigated transition on a swept plate, where crossflow was generated by a pressure gradient imposed on the plate. A review of these experiments, which approximate quasi two-dimensional flow conditions in the sense, that the laminar base flow is constant in spanwise direction, is 
given by Reed \& Saric [80]. At present, transition in swept wing flows is studied experimentally by Saric and co-workers (cf. Dagenhart et al. [17]), by Kachanov and co-workers (cf. Kachanov \& Tararykin [47]), by Bippes and co-workers (cf. Deyhle \& Bippes [18]), and by Itoh and co-workers (cf. Takagi $\&$ Itoh [92]). Recent results of these experiments are discussed in the review by Bippes [11].

In the experiments on transition in three-dimensional boundary layers, high-frequency disturbances have been observed in the region of high disturbance amplitude shortly before breakdown (see, for example, Poll [75], Kohama et al. [54], Lerche [55], Kawakami et al. [48]). Secondary high-frequency disturbances have also been studied experimentally in twodimensional flows by Klebanoff et al. [50], Bake et al. [4], Wiegel [96] and many others. With their work on stability and transition in plane Poiseuille flow, Nishioka et al. [68] contributed to a sound experimental basis for theoretical and numerical studies without the additional complication of boundary-layer growth.

Herbert [38] gives a comprehensive review on the theoretical efforts and findings concerned with secondary instability in two-dimensional boundarylayer flows. In his analysis based on Floquet theory, Herbert [38] neglects the nonlinear distortion of the primary vortex (shape assumption) and the base flow nonparallelism (parallel flow assumption). These simplifications have also been used in secondary instability analyses of three-dimensional flows by Reed [78], [79] and by Fischer \& Dallmann [22], [23]. Even though the use of the shape assumption is questionable at the rather high amplitudes necessary to observe high-frequency secondary disturbances in three-dimensional boundary layers, Fischer et al. [24] and Hein et al. [34] succeeded in showing the existence of this type of disturbances, if the primary crossflow vortices have reached high amplitudes. Balachandar et al. [5] performed theoretical studies on secondary instability of crossflow vortices on a rotating disk.

The importance of inflection points for the occurrence of high-frequency secondary disturbances in three-dimensional boundary-layers has been demonstrated using temporal direct numerical simulations by Malik [60] for the rotating disk, and by Meyer \& Kleiser [64] for the swept plate. Secondary instability of the boundary layer on the DLR swept plate has also been studied numerically by Wintergerste \& Kleiser [97] using temporal DNS, by Bonfigli \& Kloker [12] using spatial DNS, and for a Falkner-Skan-Cooke model flow by Högberg \& Henningson [42] also using spatial DNS. Apart from DNS results, nonlinear solutions of the parabolized stability equations can be used as primarily distorted base flow for secondary instability analysis. This approach has been chosen by Malik et al. [62] to generate base flows for secondary instability analyses of boundary layers on a swept wing and on swept cylinders. Using a two-dimensional eigenvalue approach, they identified two types of high-frequency modes, which correlate with spanwise and wallnormal shear of the primarily distorted base flow, respectively. Similar results 
have been found in secondary instability analyses of Görtler vortices by Li \& Malik [56], by Park \& Huerre [74], and by Bottaro \& Klingmann [13]. Also on the basis of a primarily distorted base flow computed by using the nonlinear PSE, Wang et al. [95] and Janke \& Balakumar [45] performed secondary instability analyses for swept Hiemenz flow. Koch et al. [53] used nonlinear equilibrium solutions of stationary crossflow vortices as starting point for their secondary instability analysis of the boundary layer on a swept plate. These solutions have the great advantage, that, in contrast to PSE solutions, they do not depend on initial amplitudes of the primary disturbances, i.e. on the mechanisms, that create disturbances in the boundary layer (receptivity).

\subsection{Goals of this Work}

Object of this work is a deeper understanding of instability and transition mechanisms in three-dimensional boundary-layer flows, which is a necessary prerequisite for improvements of methods for transition prediction and control. For that purpose, a model flow based on the DLR swept plate experiment is investigated for primary and secondary instability by utilizing the parabolized stability equations and Floquet theory, respectively. The definition of this model flow and the computation of the laminar base flow are discussed in chapter 2.

The primary and secondary instability analysis presented in chapters $3-5$ are focused mainly on the role of crossflow vortices in the transition process. In chapter 3, the linear PSE are derived, and some numerical aspects are discussed. The physical investigations presented in this chapter are guided by the following questions:

- To which amount do the linear PSE results depend on the initial conditions of the disturbance modes?

- Which disturbances, i.e. disturbances of which frequency and which wave numbers, exhibit the highest growth rates at different chordwise locations on the test plate?

After a brief discussion of the central aspects of the nonlinear stability equations and some introductory remarks on nonlinear disturbance growth in plane Poiseuille flow, the following questions are considered in chapter 4 for the boundary layer on the swept plate:

- What is the effect of the initial disturbance amplitude on the nonlinear evolution of these disturbances?

- How does nonlinear amplitude saturation depend on the initial conditions of disturbances? 
- What is the effect of the crossflow vortices on the steady mean flow?

- How do different disturbance modes interact? Does the interaction of disturbance modes lead to resonances, i.e. to strong mutual amplification?

- How do the nonlinear PSE results compare with nonlinear equilibrium solutions and with results from spatial DNS?

Secondary instability analysis based on Floquet theory is presented in chapter 5 . First, the underlying equations are derived, and the numerical solution procedure is sketched. The main part of this chapter is occupied by a discussion of the following questions:

- What is the threshold amplitude of the primary crossflow vortices for the occurrence of high-frequency secondary instability?

- Which properties of the primarily distorted flow field cause secondary instability?

- What is the structure of the high-frequency modes, and how do these disturbances propagate in the flow field?

Chapter 6 contains a summary of the results and some open questions. 


\section{Chapter 2}

\section{The DLR Swept Plate Experiment}

As basis for the numerical investigations, the DLR swept plate experiment is chosen. This experiment mimics quasi three-dimensional, infinite swept base flow, which significantly simplifies the theoretical treatment. A generic pressure distribution is used to define a model flow, which captures the characteristic properties of experimental data. The laminar base flow is computed using first-order boundary-layer theory. The base flow exhibits inflectional crossflow profiles and is unstable with respect to stationary and traveling disturbances.

\subsection{Experimental Setup}

The DLR swept plate experiment is specifically designed to allow detailed investigations of the transition process dominated by crossflow instability. It is conducted in Göttingen by Bippes and his group (cf. for example NitschkeKowsky \& Bippes [70], Deyhle \& Bippes [18], Lerche [55], Bippes [11]) as a basic experiment. It aims at creating a data base for theoretical and numerical analyses of the disturbance development up to the final breakdown of laminar flow as well as for transition prediction.

The experiment is performed at subsonic speeds in order to exclude compressibility effects. A three-dimensional boundary-layer is generated by means of a pressure gradient imposed by a displacement body above the plate as shown in figure 2.1. This configuration allows the study of crossflow instabilities without the influence of body curvature. Special end-plates contoured with the potential-flow streamlines help to approximate infinitespan conditions over a large region of the flow field. These conditions significantly simplify the theoretical treatment.

Due to the strong favorable, essentially constant negative pressure gradient in the direction normal to the leading edge, the flow is stable with respect to Tollmien-Schlichting instability over the whole plate. The experiments 


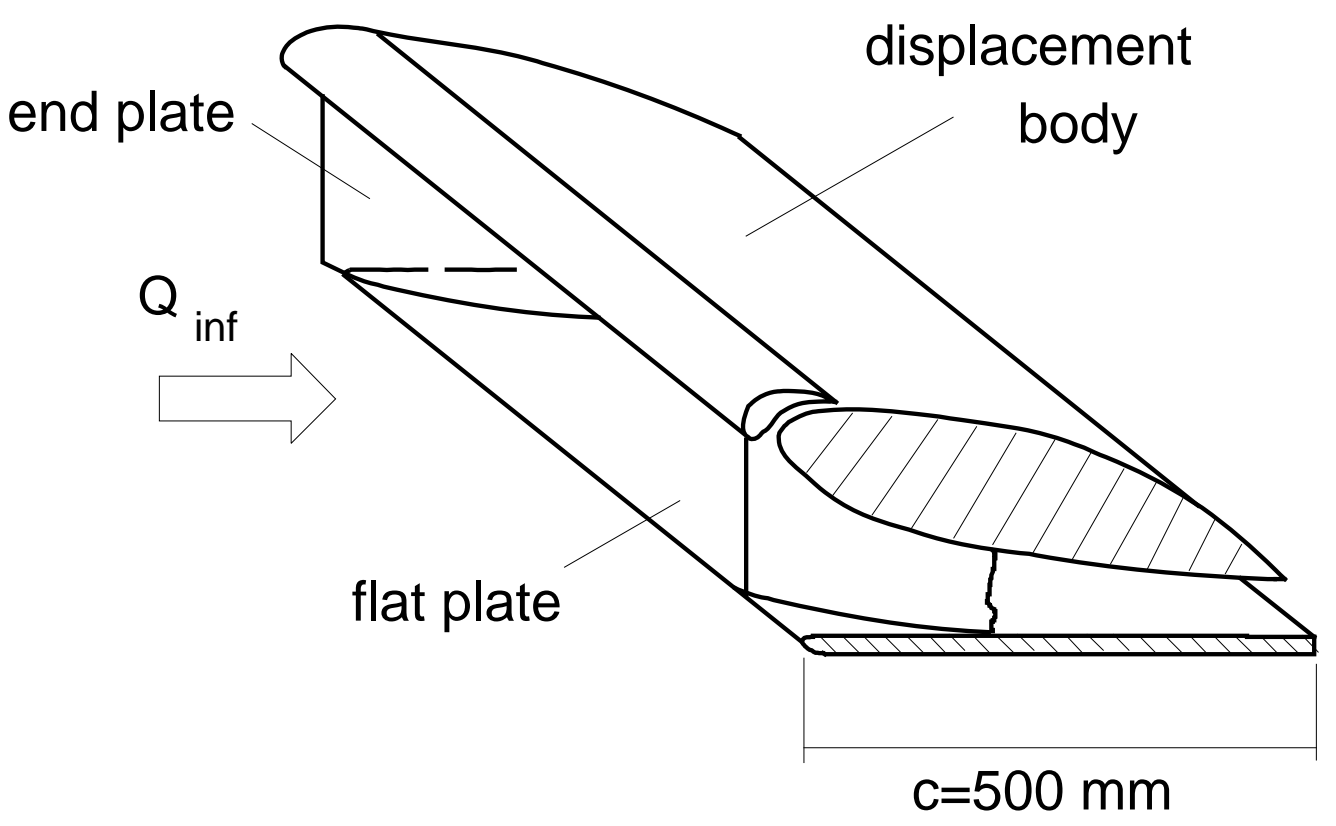

Figure 2.1: Experimental setup of DLR swept plate experiment.

are performed both under natural and controlled conditions. Parallel to the DLR swept plate experiment, main basic experimental work on crossflow instability is performed at the Arizona State University in Tempe by Saric and co-workers (cf. Dagenhart et al. [17]), at the Institute of Theoretical and Applied Mechanics in Novosibirsk by Kachanov and co-workers (cf. Kachanov \& Tararykin [47]), and at the National Aerospace Laboratory in Tokyo by Itoh and co-workers (cf. Takagi \& Itoh [92]). A detailed comparison of these experiments is given in the review by Bippes [11].

\subsection{Definition of a Model Flow}

On account of its design to support major simplifications in the flow conditions for numerical and theoretical approaches, the DLR swept plate experiment has frequently been used as data base for temporal DNS (cf. Meyer \& Kleiser [64], Meyer [63]), spatial DNS (cf. Joslin \& Streett [46], Müller [67]), calculation of nonlinear equilibrium solutions (cf. Koch et al. [53]), and PSE computations (cf. Bertolotti [8], Janke [44]). In order to specify a laminar base flow, which does not depend on small differences between different experimental tests and allows for quantitative comparisons between experimental and various theoretical and numerical results it is reasonable to define a model flow on the basis of experimental data and corresponding direct numerical simulations. Using a least-squares approximation to the experimentally measured pressure coefficient $c_{p}\left(x_{c}\right)$ from Müller \& Bippes [66], Meyer [63] analytically describes the pressure distribution for $x_{c}>0.04$ by 
the linear function

$$
c_{p}\left(x_{c}\right)=0.941-0.845 x_{c},
$$

where $x_{c}$ denotes the nondimensional chordwise coordinate (see section 2.3). This definition is also used in the spatial simulations by Joslin \& Streett [46] and Müller [67]. For the nonsimilar boundary-layer computation used in our analysis it is necessary to include the attachment line, where $c_{p}$ is one by definition. In the vicinity of the attachment line the pressure coefficient is approximated by a parabola. In agreement with Müller \& Bippes [66] and Lerche [55] we assume the attachment line to be at $x_{c}=0.02$ on the top side of the plate. In order to obtain a pressure distribution which is continuous up to second order, we supplement the approximation (2.1) as follows (see figure 2.2):

$$
\begin{aligned}
\text { Region I }\left(x_{c} \leq 0.04\right): & & c_{p}\left(x_{c}\right)=a\left(x_{c}-0.02\right)^{2}+1 \\
\text { Region II }\left(0.04 \leq x_{c} \leq 0.1\right): & & c_{p}\left(x_{c}\right)=b x_{c}^{4}+c x_{c}^{3}+d x_{c}^{2}+e x_{c}+f(2.2) \\
\text { Region III } \quad\left(x_{c} \geq 0.1\right): & & c_{p}\left(x_{c}\right)=0.941-0.845 x_{c}
\end{aligned}
$$

The numerical values for the coefficients are obtained from the requirement of $c_{p}$ and its first two derivatives to be continuous at $x_{c}=0.04$ and $x_{c}=0.1$ :

$$
\begin{array}{r}
a=-53.7045, \quad b=-10475.6, \quad c=3231.52, \quad d=-340.922, \\
e=12.296, \quad f=0.852153
\end{array}
$$
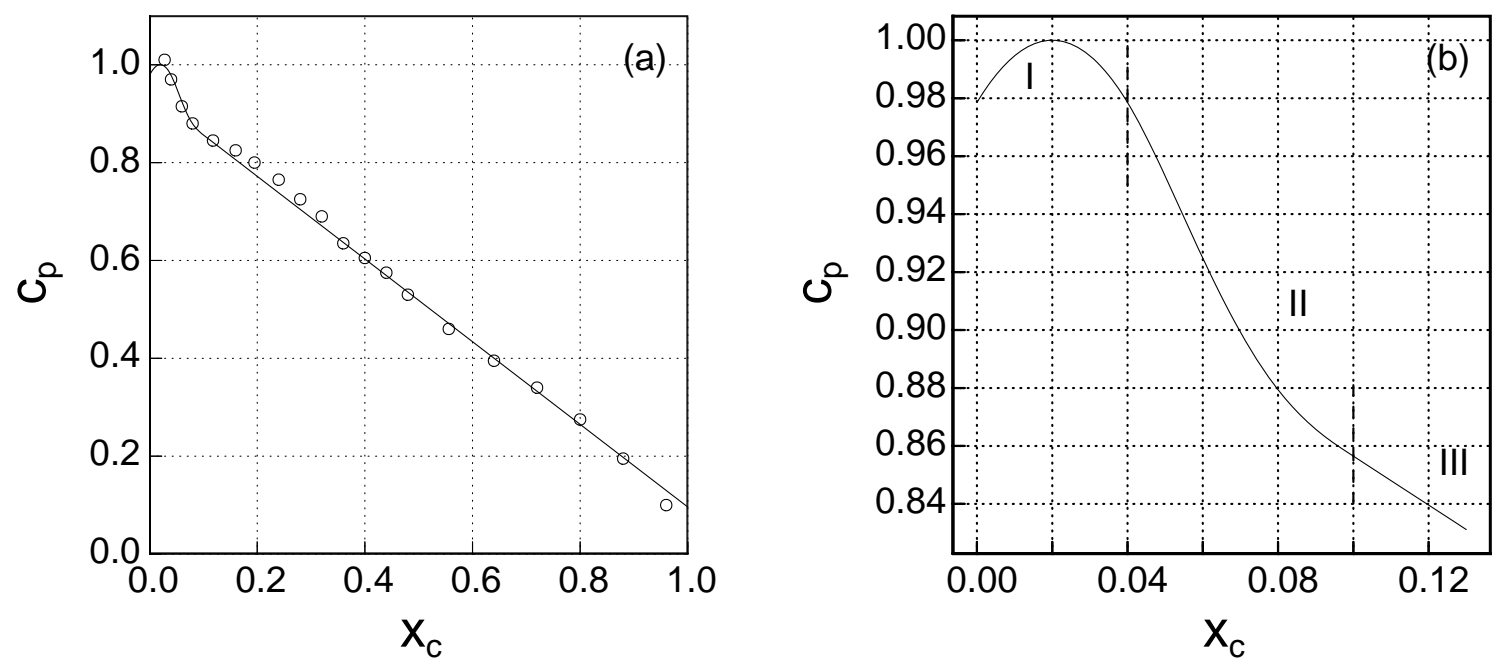

Figure 2.2: (a) Analytical approximation (2.2) of pressure distribution (o) on the DLR swept plate experiment (Müller \& Bippes [66]). (b) Analytical pressure distribution (2.2) near the attachment line. 


\subsection{The Laminar Base Flow}

In this section the parameters of the investigated flow field are given, and the calculation of the laminar base flow is outlined. The boundary-layer calculation is performed in the body-fixed chordwise coordinate system $\left(x_{c}^{*}, y^{*}, z_{c}^{*}\right)$ which is shown in figure 2.3 together with the vortex-oriented coordinate system $\left(x_{\psi}^{*}, y^{*}, z_{\psi}^{*}\right)$ used in the secondary instability analysis.

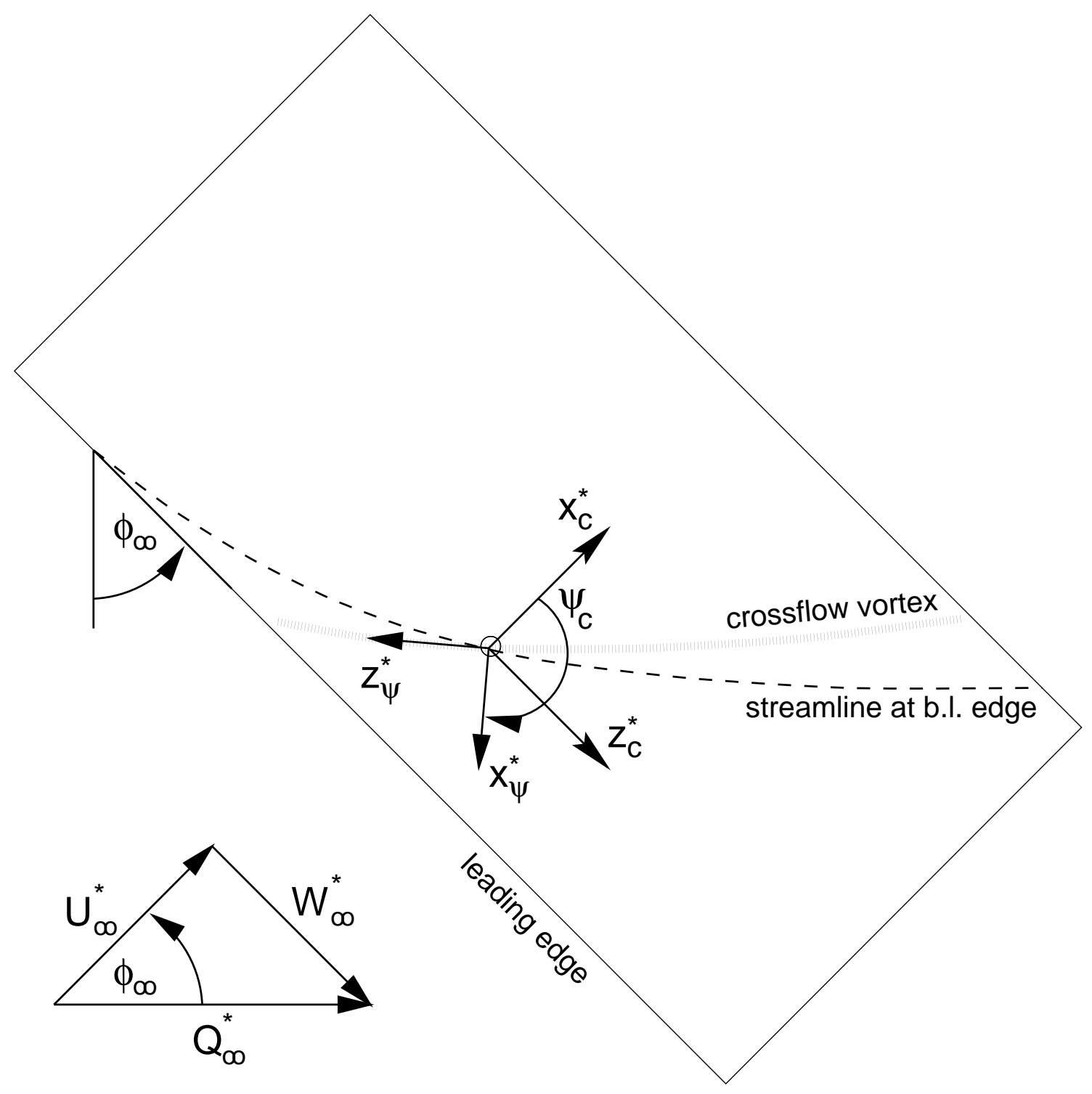

Figure 2.3: Body fixed and vortex-oriented coordinate system.

The chord length of the plate is $c^{*}=0.5 \mathrm{~m}$, and the free stream velocity is $Q_{\infty}^{*}=19 \frac{\mathrm{m}}{\mathrm{s}}$. The geometric sweep angle of the plate is $45^{\circ}$, but on account of the displacement effect of the wind-tunnel model an effective sweep angle $\phi_{\infty}=42.5^{\circ}$ is used in our calculations. The properties of air at standard conditions are taken from Dubbel [20] and summarized in table 2.1. 


\begin{tabular}{||c|c||}
\hline \hline$\rho_{\infty}^{*}$ & $1.21 \frac{\mathrm{kg}}{\mathrm{m}^{3}}$ \\
\hline$\nu_{\infty}^{*}=\frac{\mu_{\infty}^{*}}{\rho_{\infty}^{*}}$ & $1.51 \cdot 10^{-5} \frac{\mathrm{m}^{2}}{\mathrm{~s}}$ \\
\hline$\mu_{\infty}^{*}$ & $1.83 \cdot 10^{-5} \frac{\mathrm{kg}}{\mathrm{ms}}$ \\
\hline$c^{*}$ & $0.5 \mathrm{~m}$ \\
\hline$Q_{\infty}^{*}$ & $19.0 \frac{\mathrm{m}}{\mathrm{s}}$ \\
\hline$\phi_{\infty}$ & $42.5^{\circ}$ \\
\hline \hline
\end{tabular}

Table 2.1: Free-stream quantities of DLR swept plate experiment.

Due to the infinite swept wing assumption the spanwise velocity component at the boundary-layer edge

$$
W_{c, e}^{*}=Q_{\infty}^{*} \sin \phi_{\infty}
$$

is constant, and the chordwise component

$$
U_{c, e}^{*}=Q_{\infty}^{*} \cos \phi_{\infty} \sqrt{1-c_{p}}
$$

depends on $x_{c}^{*}$ only.

Since the base flow velocity components do not depend on the spanwise coordinate $z_{c}^{*}$, the steady, incompressible boundary-layer equations (cf. e.g. Schlichting [84], chapter 7.2) can be written as follows:

$$
\begin{aligned}
U_{c}^{*} \frac{\partial U_{c}^{*}}{\partial x_{c}^{*}}+V^{*} \frac{\partial U_{c}^{*}}{\partial y^{*}} & =U_{c, e}^{*} \frac{d U_{c, e}^{*}}{d x_{c}^{*}}+\nu^{*} \frac{\partial^{2} U_{c}^{*}}{\partial y^{* 2}} \\
U_{c}^{*} \frac{\partial W_{c}^{*}}{\partial x_{c}^{*}}+V^{*} \frac{\partial W_{c}^{*}}{\partial y^{*}} & =\nu^{*} \frac{\partial^{2} W_{c}^{*}}{\partial y^{* 2}} \\
\frac{\partial U_{c}^{*}}{\partial x_{c}^{*}}+\frac{\partial V^{*}}{\partial y^{*}} & =0
\end{aligned}
$$

with boundary conditions

$$
y^{*}=0: \quad U_{c}^{*}=V^{*}=W_{c}^{*}=0 ; \quad y^{*} \rightarrow \infty: \quad U_{c}^{*}=U_{c, e}^{*}, \quad W_{c}^{*}=W_{c, e}^{*} .
$$

The continuity equation (2.8) is integrated by introducing a stream function $\Psi^{*}\left(x_{c}^{*}, y^{*}\right)$ such that

$$
U_{c}^{*}=\frac{\partial \Psi^{*}}{\partial y^{*}}, \quad V^{*}=-\frac{\partial \Psi^{*}}{\partial x_{c}^{*}} .
$$

Hence, (2.6) and (2.7) read

$$
\begin{aligned}
\frac{\partial \Psi^{*}}{\partial y^{*}} \frac{\partial^{2} \Psi^{*}}{\partial x_{c}^{*} \partial y^{*}}-\frac{\partial \Psi^{*}}{\partial x_{c}^{*}} \frac{\partial^{2} \Psi^{*}}{\partial y^{* 2}} & =U_{c, e}^{*} \frac{d U_{c, e}^{*}}{d x_{c}^{*}}+\nu^{*} \frac{\partial^{3} \Psi^{*}}{\partial y^{* 3}} \\
\frac{\partial \Psi^{*}}{\partial y^{*}} \frac{\partial W_{c}^{*}}{\partial x_{c}^{*}}-\frac{\partial \Psi^{*}}{\partial x_{c}^{*}} \frac{\partial W_{c}^{*}}{\partial y^{*}} & =\nu^{*} \frac{\partial^{2} W_{c}^{*}}{\partial y^{* 2}} .
\end{aligned}
$$


We now transform $x_{c}^{*}, y^{*}$, and $z_{c}^{*}$ into nondimensional form by introducing a local similarity variable $\chi$ (cf. Koch et al. [53]) for the wallnormal coordinate, while the chordwise and spanwise coordinates are nondimensionalized with the chord length $c^{*}$ of the plate:

$$
x_{c}=\frac{1}{c^{*}} x_{c}^{*}, \quad \chi=\sqrt{\frac{U_{c, e}^{*}\left(x_{c}^{*}\right)}{\nu^{*} x_{c}^{*}}} y^{*}, \quad z_{c}=\frac{1}{c^{*}} z_{c}^{*} .
$$

The stream function and the spanwise velocity component are then made dimensionless by the substitutions

$$
\Psi^{*}\left(x_{c}, \chi\right)=\sqrt{c^{*} x_{c} \nu^{*} U_{c, e}^{*}\left(x_{c}\right)} F\left(x_{c}, \chi\right)
$$

and

$$
W_{c}^{*}\left(x_{c}, \chi\right)=W_{c, e}^{*} G\left(x_{c}, \chi\right) .
$$

Introducing (2.14) and (2.15) into (2.11) and (2.12) yields

$$
F^{\prime \prime \prime}+\frac{1+m}{2} F F^{\prime \prime}+m\left(1-F^{2}\right)=x_{c}\left(F^{\prime} \frac{\partial F^{\prime}}{\partial x_{c}}-F^{\prime \prime} \frac{\partial F}{\partial x_{c}}\right)
$$

and

$$
G^{\prime \prime}+\frac{1+m}{2} F G^{\prime}=x_{c}\left(F^{\prime} \frac{\partial G}{\partial x_{c}}-G^{\prime} \frac{\partial F}{\partial x_{c}}\right)
$$

where

$$
\begin{aligned}
& U_{c, e}=\frac{1}{Q_{\infty}^{*}} U_{c, e}^{*}, \\
& m=\frac{x_{c}}{U_{c, e}} \frac{\partial U_{c, e}}{\partial x_{c}},
\end{aligned}
$$

and primes denote differentiation with respect to $\chi$.

The boundary conditions are:

$$
\chi=0: \quad F=\frac{\partial F}{\partial \chi}=G=0 ; \quad \chi \rightarrow \infty: \quad \frac{\partial F}{\partial \chi}=G=1 .
$$

The starting solution for the boundary-layer calculation is obtained by solving the swept Hiemenz equations

$$
\begin{gathered}
F^{\prime \prime \prime}+F F^{\prime \prime}+\left(1-F^{2}\right)=0, \\
G^{\prime \prime}+F G^{\prime}=0
\end{gathered}
$$

at the attachment line. The nonsimilar equations (2.16) and (2.17) are then solved by a downstream marching procedure. For that, a spectral multidomain boundary-layer code by Bertolotti [7] employing a first-order Euler 

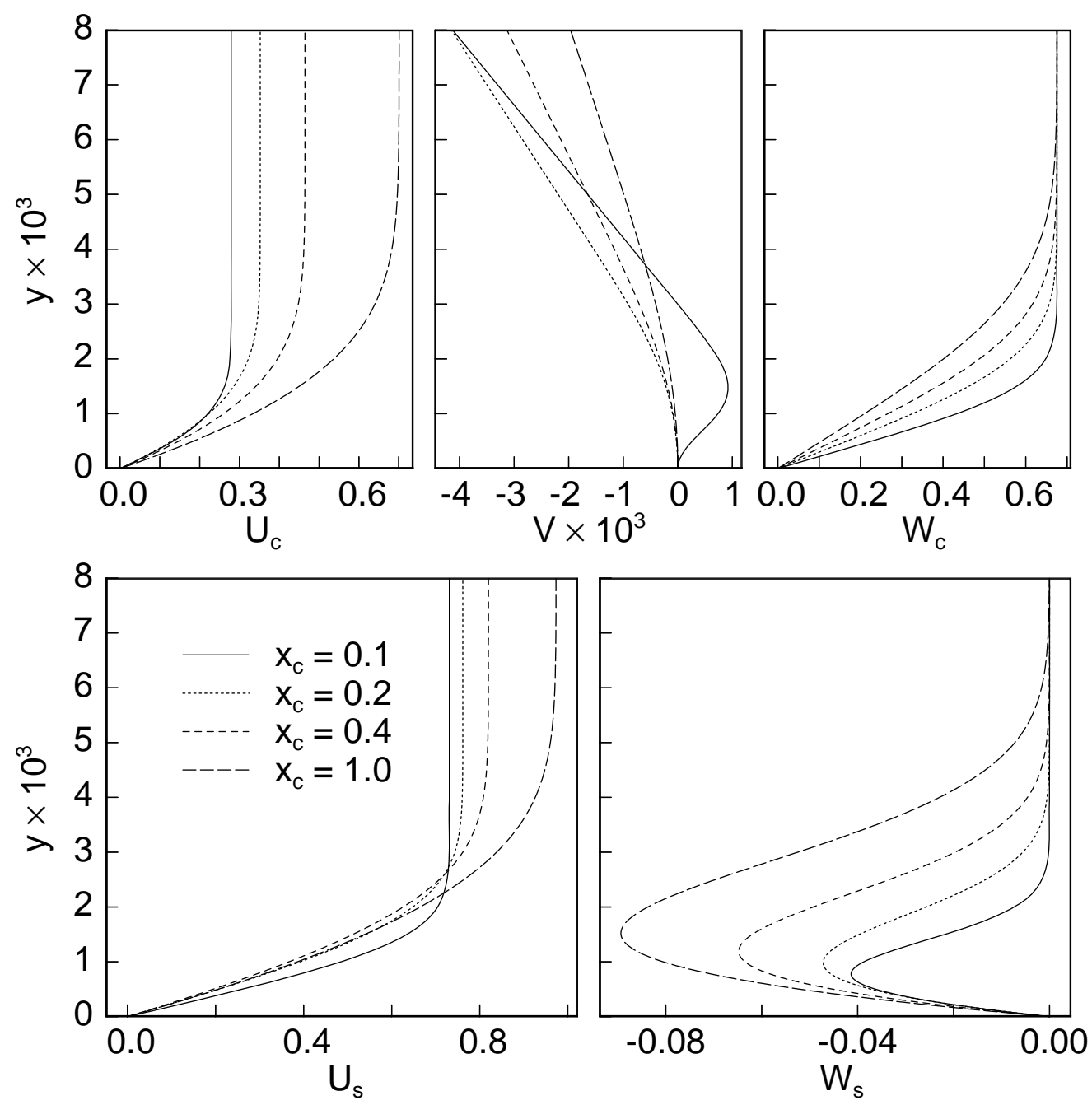

Figure 2.4: Nonsimilar boundary-layer profiles for various $x_{c}$-positions in chordwise coordinates (top row) and in a coordinate system $\left(x_{s}, y, z_{s}\right)$, where $x_{s}$ is in the direction of the local free-stream velocity at the boundary-layer edge (bottom row).

discretization of the $x_{c}$-derivatives is used. Boundary-layer profiles at different chordwise positions are shown in figure 2.4. Here and in the following chapters the wallnormal $y$-coordinate is nondimensionalized w.r.t. the chord length $c^{*}$ for the presentation of numerical results.

As can be seen from the continuity equation (2.8), the velocity $V$ normal to the wall is not constant outside the boundary layer if $\frac{d U_{c, e}}{d x_{c}} \neq 0$. However, numerical experiments (cf. Stolte et al. [91]) have shown that the behavior of $V$ far away from the wall does not have noticeable influence on the instability properties of the flow field.

While the spanwise velocity component at the boundary-layer edge $W_{c, e}$ is independent of $x_{c}$, the chordwise component $U_{c, e}$ increases with $x_{c}$. This leads to curved potential streamlines with an equilibrium between centrifu- 
gal forces and pressure forces due to the pressure gradient component normal to the potential streamline. Inside the boundary layer, the flow velocity is reduced by viscosity, while the pressure gradient is constant within the boundary-layer approximation. In order to maintain the balance of forces, stronger curvature of streamlines inside the boundary layer arises. This leads to a flow perpendicular to the potential streamline, the crossflow $W_{s}$. Due to the inflection point, which is naturally present in crossflow profiles, such flows are inviscidly unstable with respect to stationary and traveling disturbances (cf. Drazin \& Reid [19]). The evolution of these disturbances in the boundary layer is investigated in detail in the following chapters. 


\section{Chapter 3}

\section{Linear Nonlocal Instability Analysis}

The linear parabolized stability equations (PSE) capture the spatial evolution of convectively unstable disturbances in weakly nonparallel flows. Residual ellipticity of these equations leads to a lower limit for the step size used for the downstream marching. By neglecting the pressure term from the $u$-momentum equation, the main part of the ellipticity (and, thus, the step size limit) can be removed. Different approaches to specify initial conditions for the PSE are compared in section 3.4. The most complete nonparallel approach is then used for linear, parametric instability investigations. The frequencies and wave numbers of the most unstable modes agree well with experimental observations and previous numerical studies.

\subsection{Introduction}

Nonlocal instability analysis based on the parabolized stability equations (PSE) is an approach to analyze the streamwise evolution of convectively unstable disturbances in weekly nonparallel flows. The method has been developed by Herbert and Bertolotti (cf. Bertolotti [6]) and independently by Dallmann and Simen (cf. Simen [87]) and can be positioned between strongly simplified, but efficient, classical local instability analysis (cf. Mack [59]) and the complete, but computationally very expensive, flow-field analysis via direct numerical simulation. Nonlocal instability means that the growth rates of disturbances are not only influenced by the local flow conditions, but also by their local gradient in the direction of spatial amplification, i.e. nonlocal refers to quantitative effects on disturbance growth rather than new or different nonlocal mechanisms causing any type of otherwise absent disturbance amplification. The concept rests on the decomposition of disturbance modes into amplitude function and wave function, where both amplitude function and wave number vary only slowly in downstream direction. Hence, second derivatives of the slowly varying amplitude functions with respect to 
the streamwise variable can be neglected leading to an initial-boundaryvalue problem, which can be solved efficiently by numerical marching procedures.

\subsection{The Parabolized Stability Equations}

Based on the exposition in Herbert [39] the derivation of the PSE is outlined in this section for incompressible boundary-layer flows, where density $\rho$ and viscosity $\mu$ of the fluid are constants. For the flow over the infinite swept plate described in chapter 2, the conservation equations for momentum and mass can be written as follows:

$$
\begin{aligned}
\frac{\partial u_{c}}{\partial t}+u_{c} \frac{\partial u_{c}}{\partial x_{c}}+v \frac{\partial u_{c}}{\partial y}+w_{c} \frac{\partial u_{c}}{\partial z_{c}} & =-\frac{\partial p}{\partial x_{c}}+\frac{1}{R e} \Delta_{c} u_{c} \\
\frac{\partial v}{\partial t}+u_{c} \frac{\partial v}{\partial x_{c}}+v \frac{\partial v}{\partial y}+w_{c} \frac{\partial v}{\partial z_{c}} & =-\frac{\partial p}{\partial y}+\frac{1}{R e} \Delta_{c} v \\
\frac{\partial w_{c}}{\partial t}+u_{c} \frac{\partial w_{c}}{\partial x_{c}}+v \frac{\partial w_{c}}{\partial y}+w_{c} \frac{\partial w_{c}}{\partial z_{c}} & =-\frac{\partial p}{\partial z_{c}}+\frac{1}{R e} \Delta_{c} w_{c} \\
\frac{\partial u_{c}}{\partial x_{c}}+\frac{\partial v}{\partial y}+\frac{\partial w_{c}}{\partial z_{c}} & =0
\end{aligned}
$$

where

$$
\Delta_{c}=\frac{\partial^{2}}{\partial x_{c}^{2}}+\frac{\partial^{2}}{\partial y^{2}}+\frac{\partial^{2}}{\partial z_{c}^{2}}
$$

and

$$
R e=\frac{Q_{\infty}^{*} c^{*}}{\nu^{*}}
$$

The total flow field $\mathbf{q}=\left(u_{c}, v, w_{c}, p\right)^{T}$ is decomposed into the steady laminar base flow $\mathbf{Q}=\left(U_{c}, V, W_{c}, P\right)^{T}$ and the disturbance $\tilde{\mathbf{q}}=\left(\tilde{u}_{c}, \tilde{v}, \tilde{w}_{c}, \tilde{p}\right)^{T}$ :

$$
\mathbf{q}\left(x_{c}, y, z_{c}, t\right)=\mathbf{Q}\left(x_{c}, y\right)+\tilde{\mathbf{q}}\left(x_{c}, y, z_{c}, t\right) .
$$

The decomposition (3.7) is now introduced into (3.1) - (3.4) and the equations satisfied by the laminar basic flow are subtracted:

$$
\begin{gathered}
\frac{\partial \tilde{u}_{c}}{\partial t}+U_{c} \frac{\partial \tilde{u}_{c}}{\partial x_{c}}+\tilde{u}_{c} \frac{\partial U_{c}}{\partial x_{c}}+V \frac{\partial \tilde{u}_{c}}{\partial y}+\tilde{v} \frac{\partial U_{c}}{\partial y}+W_{c} \frac{\partial \tilde{u}_{c}}{\partial z_{c}}+\frac{\partial \tilde{p}}{\partial x_{c}}-\frac{1}{R e} \Delta_{c} \tilde{u}_{c} \\
=-\left(\tilde{u}_{c} \frac{\partial \tilde{u}_{c}}{\partial x_{c}}+\tilde{v} \frac{\partial \tilde{u}_{c}}{\partial y}+\tilde{w}_{c} \frac{\partial \tilde{u}_{c}}{\partial z_{c}}\right) \\
\frac{\partial \tilde{v}}{\partial t}+U_{c} \frac{\partial \tilde{v}}{\partial x_{c}}+\tilde{u}_{c} \frac{\partial V}{\partial x_{c}}+V \frac{\partial \tilde{v}}{\partial y}+\tilde{v} \frac{\partial V}{\partial y}+W_{c} \frac{\partial \tilde{v}}{\partial z_{c}}+\frac{\partial \tilde{p}}{\partial y}-\frac{1}{R e} \Delta_{c} \tilde{v} \\
=-\left(\tilde{u}_{c} \frac{\partial \tilde{v}}{\partial x_{c}}+\tilde{v} \frac{\partial \tilde{v}}{\partial y}+\tilde{w}_{c} \frac{\partial \tilde{v}}{\partial z_{c}}\right)
\end{gathered}
$$




$$
\begin{aligned}
\frac{\partial \tilde{w}_{c}}{\partial t}+U_{c} \frac{\partial \tilde{w}_{c}}{\partial x_{c}}+\tilde{u}_{c} \frac{\partial W_{c}}{\partial x_{c}}+ & V \frac{\partial \tilde{w}_{c}}{\partial y}+\tilde{v} \frac{\partial W_{c}}{\partial y}+W_{c} \frac{\partial \tilde{w}_{c}}{\partial z_{c}}+\frac{\partial \tilde{p}}{\partial z_{c}}-\frac{1}{R e} \Delta_{c} \tilde{w}_{c} \\
=- & \left(\tilde{u}_{c} \frac{\partial \tilde{w}_{c}}{\partial x_{c}}+\tilde{v} \frac{\partial \tilde{w}_{c}}{\partial y}+\tilde{w}_{c} \frac{\partial \tilde{w}_{c}}{\partial z_{c}}\right) \\
& \frac{\partial \tilde{u}_{c}}{\partial x_{c}}+\frac{\partial \tilde{v}}{\partial y}+\frac{\partial \tilde{w}_{c}}{\partial z_{c}}=0
\end{aligned}
$$

For a linear nonlocal instability analysis the nonlinear terms

$$
\begin{aligned}
& N_{1}=\tilde{u}_{c} \frac{\partial \tilde{u}_{c}}{\partial x_{c}}+\tilde{v} \frac{\partial \tilde{u}_{c}}{\partial y}+\tilde{w}_{c} \frac{\partial \tilde{u}_{c}}{\partial z_{c}} \\
& N_{2}=\tilde{u}_{c} \frac{\partial \tilde{v}}{\partial x_{c}}+\tilde{v} \frac{\partial \tilde{v}}{\partial y}+\tilde{w}_{c} \frac{\partial \tilde{v}}{\partial z_{c}} \\
& N_{3}=\tilde{u}_{c} \frac{\partial \tilde{w}_{c}}{\partial x_{c}}+\tilde{v} \frac{\partial \tilde{w}_{c}}{\partial y}+\tilde{w}_{c} \frac{\partial \tilde{w}_{c}}{\partial z_{c}}
\end{aligned}
$$

are neglected in (3.8) - (3.10) and the disturbance $\tilde{\mathbf{q}}$ is decomposed into an amplitude function $\hat{\mathbf{q}}$ and a wave function $\chi$ :

$$
\tilde{\mathbf{q}}\left(x_{c}, y, z_{c}, t\right)=\hat{\mathbf{q}}\left(x_{c}, y\right) \chi\left(x_{c}, z_{c}, t\right)+c . c .,
$$

where c.c. denotes the complex conjugate, and

$$
\chi\left(x_{c}, z_{c}, t\right)=e^{i\left[\theta_{c}\left(x_{c}\right)+\beta_{c} z_{c}-\omega t\right]}
$$

with

$$
\frac{d \theta_{c}\left(x_{c}\right)}{d x_{c}}=\alpha_{c}\left(x_{c}\right) .
$$

Here $\alpha_{c}$ denotes the chordwise, $\beta_{c}$ the spanwise wavenumber of the disturbance, $\omega$ denotes the angular frequency.

The substitution of (3.15) into the linearized form of (3.8) - (3.11) yields

$$
\begin{gathered}
\left\{-i \omega+i \alpha_{c} U_{c}+\frac{\partial U_{c}}{\partial x_{c}}+V \frac{\partial}{\partial y}+i \beta_{c} W_{c}-\frac{1}{R e}\left[\frac{\partial^{2}}{\partial y^{2}}-\left(\alpha_{c}^{2}+\beta_{c}^{2}\right)\right]\right\} \hat{u}_{c} \\
+\frac{\partial U_{c}}{\partial y} \hat{v}+i \alpha_{c} \hat{p}+\left\{U_{c}-\frac{2 i \alpha_{c}}{R e}\right\} \frac{\partial \hat{u}_{c}}{\partial x_{c}}+\frac{\partial \hat{p}}{\partial x_{c}}-\frac{i}{R e} \frac{d \alpha_{c}}{d x_{c}} \hat{u}_{c}=\frac{1}{R e} \frac{\partial^{2} \hat{u}_{c}}{\partial x_{c}^{2}} \\
\left\{-i \omega+i \alpha_{c} U_{c}+V \frac{\partial}{\partial y}+\frac{\partial V}{\partial y}+i \beta_{c} W_{c}-\frac{1}{R e}\left[\frac{\partial^{2}}{\partial y^{2}}-\left(\alpha_{c}^{2}+\beta_{c}^{2}\right)\right]\right\} \hat{v} \\
+\frac{\partial V}{\partial x_{c}} \hat{u}_{c}+\frac{\partial \hat{p}}{\partial y}+\left\{U_{c}-\frac{2 i \alpha_{c}}{R e}\right\} \frac{\partial \hat{v}}{\partial x_{c}}-\frac{i}{R e} \frac{d \alpha_{c}}{d x_{c}} \hat{v}=\frac{1}{R e} \frac{\partial^{2} \hat{v}}{\partial x_{c}^{2}} \\
\left\{-i \omega+i \alpha_{c} U_{c}+V \frac{\partial}{\partial y}+i \beta_{c} W_{c}-\frac{1}{R e}\left[\frac{\partial^{2}}{\partial y^{2}}-\left(\alpha_{c}^{2}+\beta_{c}^{2}\right)\right]\right\} \hat{w}_{c} \\
+\frac{\partial W_{c}}{\partial x_{c}} \hat{u}_{c}+\frac{\partial W_{c}}{\partial y} \hat{v}+i \beta_{c} \hat{p}+\left\{U_{c}-\frac{2 i \alpha_{c}}{R e}\right\} \frac{\partial \hat{w}_{c}}{\partial x_{c}}-\frac{i}{R e} \frac{d \alpha_{c}}{d x_{c}} \hat{w}_{c}=\frac{1}{R e} \frac{\partial^{2} \hat{w}_{c}}{\partial x_{c}^{2}} \\
i \alpha_{c} \hat{u}_{c}+\frac{\partial \hat{v}}{\partial y}+i \beta_{c} \hat{w}_{c}+\frac{\partial \hat{u}_{c}}{\partial x_{c}}=0
\end{gathered}
$$


If the wave number $\alpha_{c}\left(x_{c}\right)$ properly captures the oscillatory behavior of the disturbance in chordwise direction, both the amplitude function $\hat{\mathbf{q}}$ and the wave number $\alpha_{c}$ change only slowly in $x_{c}$-direction. The central assumption in the PSE-concept is, that second $x_{c}$-derivatives of $\hat{\mathbf{q}}$ are sufficiently small to be neglected. In (3.18) - (3.20) these are the terms on the right hand side of the equations. The structure of the remaining system can be surveyed more easily if it is written in the form

$$
L \hat{\mathbf{q}}+M \frac{\partial \hat{\mathbf{q}}}{\partial x_{c}}+\frac{d \alpha_{c}}{d x_{c}} N \hat{\mathbf{q}}=0,
$$

where the operators $L, M$, and $N$ act only in y.

The boundary conditions for disturbances are homogeneous at the wall and at the boundary-layer edge:

$$
y=0: \quad \hat{u}_{c}=\hat{v}=\hat{w}_{c}=0 ; \quad y \rightarrow \infty: \quad \hat{u}_{c} \rightarrow 0, \quad \hat{w}_{c} \rightarrow 0, \quad \hat{p} \rightarrow 0
$$

To start the integration of the PSE (3.22), initial conditions are needed. These are obtained using a procedure provided by Bertolotti [6], which is briefly described in section 3.4. Disturbance growth is calculated using spatial theory, i.e.

$$
\Im(\omega)=: \omega_{i}=0 .
$$

Due to the steady base flow and the infinite swept wing assumption, the angular frequency $\omega_{r}$ and the spanwise wave number $\beta_{c, r}$ do not depend on $x_{c}$ :

$$
\begin{gathered}
\Re(\omega)=: \omega_{r}=\text { const. } \\
\Re\left(\beta_{c}\right)=: \beta_{c, r}=\text { const. }
\end{gathered}
$$

Since the disturbance is not allowed to grow indefinitely in spanwise direction, it is necessary to require

$$
\Im\left(\beta_{c}\right)=: \beta_{c, i}=0 .
$$

In order to obtain a solution to (3.22), it is necessary to remove the ambiguity in the partition (3.15) - (3.17), where both unknowns $\alpha_{c}$ and $\hat{\mathbf{q}}$ depend on $x_{c}$. This is done by means of an additional equation, the so-called "normalization condition" or "auxiliary condition"

$$
\int_{0}^{y_{e}} \hat{u}_{c}^{\dagger} \frac{\partial \hat{u}_{c}}{\partial x_{c}} d y=0,
$$

where the dagger denotes the complex conjugate and $y_{e}$ the $y$-coordinate at the upper end of the discretized domain. Equation (3.28) minimizes the change of $\hat{u}_{c}$ in $x_{c}$-direction in a weighted sense across the discretized $y$ domain and, hence, keeps the error due to the PSE-assumption small (see Herbert [39]). 
Another consequence of the $x_{c}$-dependence of the amplitude function $\hat{\mathbf{q}}$ is, that - in contrast to classical local theory - the amplitude function contributes to physical wave number $\alpha_{c, p h y s}$ and growth rate $\gamma_{c, \text { phys }}$ :

$$
\alpha_{c, p h y s}=\alpha_{c, r}+\Im\left(\frac{1}{\hat{q}} \frac{\partial \hat{q}}{\partial x_{c}}\right)
$$

and

$$
\gamma_{c, p h y s}=-\alpha_{c, i}+\Re\left(\frac{1}{\hat{q}} \frac{\partial \hat{q}}{\partial x_{c}}\right),
$$

where $\hat{q}$ denotes any component of $\hat{\mathbf{q}}$. Thus, physical growth rate and wave number of a disturbance depend on the choice of $\hat{q}$ and on the considered $y$-location in the boundary layer (cf. Bertolotti [6], Stolte et al. [91]). In the present work, the calculation of $\alpha_{c, \text { phys }}$ and $\gamma_{c, p h y s}$ is based on the maximum (in $y$-direction) of the chordwise velocity component $\hat{u}_{c, \text { max }}$, unless explicitly mentioned.

From (3.27) it is obvious, that disturbances are permitted only to grow (or decay) in chordwise, i. e. in $x_{c}$-direction. The local direction of wave propagation or orientation of vortices $\psi_{c}$ is given by

$$
\psi_{c}=\arctan \left(\frac{\beta_{c}}{\alpha_{c, p h y s}}\right) .
$$

\subsection{Numerical Aspects}

For the linear and nonlinear PSE calculations and for the generation of initial conditions, the XPSE toolkit by Bertolotti [7] is used. An important aspect of this toolkit is the strict separation of the code into user-defined subroutines containing the physics of the flow and a library of numerical methods. In the wallnormal $y$-direction, a spectral collocation method with multiple sub-domains is used, while for the $x_{c}$-discretization a backward-Euler finite difference formulation is employed.

The PSE (3.22) are parabolic in the sense that they do not contain second or higher derivatives in $x_{c}$-direction. However, numerical instabilities occur for small marching steps and indicate a residual ellipticity of the equations (cf. Chang et al. [16], Haj-Hariri [31], Li \& Malik [57]). The minimal possible marching step size depends on the $x_{c}$-discretization scheme and on the choice of the auxiliary condition (cf. Hein et al. [35]). Nevertheless, the criterion given by Li \& Malik [57],

$$
\Delta x_{c}>\frac{1}{\left|\alpha_{c, p h y s}\right|},
$$

gives good orientation for the step size limit. For the laminar base flow of the DLR swept plate experiment as described in chapter 2 and a crossflow 
vortex with spanwise wave length $\lambda_{z, c}^{*}=12 \mathrm{~mm}$ (i.e. $\beta_{c}=261.8$ ), which is investigated in detail in the following sections, linear PSE calculations with different step sizes are shown in figures 3.1 and 3.2.

As can be seen from figure 3.1, the smallest possible step size, where no numerical instability occurs, is $\Delta x_{c}=0.004$. Since the smallest $\left|\alpha_{c, p h y s}\right|$ for the considered disturbance is 220 at the end of the plate, criterion (3.32) is confirmed. Further upstream, $\left|\alpha_{c, \text { phys }}\right|$ is greater, and smaller steps can be used. The wave numbers at breakdown for different step sizes are summarized in table 3.1.

One possibility to remove the (main part of the) residual ellipticity from the PSE is to neglect the $\partial \hat{p} / \partial x_{c}$-term from the $u$-momentum equation. This approximation practically removes the lower step size limit. Figure 3.2 shows the influence of the $\partial \hat{p} / \partial x_{c}$-term on the growth rate of the considered crossflow vortex. Neglecting this term leads to slightly stronger disturbance amplification. Downstream of $x_{c}=0.3$ the influence of the $\partial \hat{p} / \partial x_{c}$-term on the growth rate of the vortex is below $1 \%$. Thus, it will be neglected for convenience in the following.

For very small step sizes strong oscillations of the growth rate can be observed in the front part of the plate (figure 3.2). However, these oscillations are not caused by the ill-posedness of the problem due to residual ellipticity in the equations, but rather a transient phenomenon. Different step sizes lead to significantly different results. The validity of the PSE assumption is questionable in this region, where the basic flow changes rather rapidly. In addition to this, transient behavior is caused by the initial conditions, which do not satisfy the PSE. It is possible to reduce these transients by means of small modifications of the pressure distribution and, thus, the laminar base flow, but there is no physical justification to do so. Fortunately, these transients disappear further downstream and do not render the discussion of nonlinear and secondary instability more difficult. The generation of initial conditions is outlined in the next section.

\begin{tabular}{||c|c|c||}
\hline \hline$\Delta x_{c}$ & $\left|\alpha_{c, \text { phys }}\right|$ at breakdown & $\Delta x_{c} \cdot\left|\alpha_{c, \text { phys }}\right|$ \\
\hline \hline 0.001 & 640 & 0.64 \\
\hline 0.002 & 410 & 0.82 \\
\hline 0.003 & 270 & 0.81 \\
\hline 0.004 & $<220$ & $<0.88$ \\
\hline \hline
\end{tabular}

Table 3.1: Lower step size limits for PSE marching procedure (see criterion 3.32). Considered is a crossflow vortex with $\beta_{c}=261.8$. 


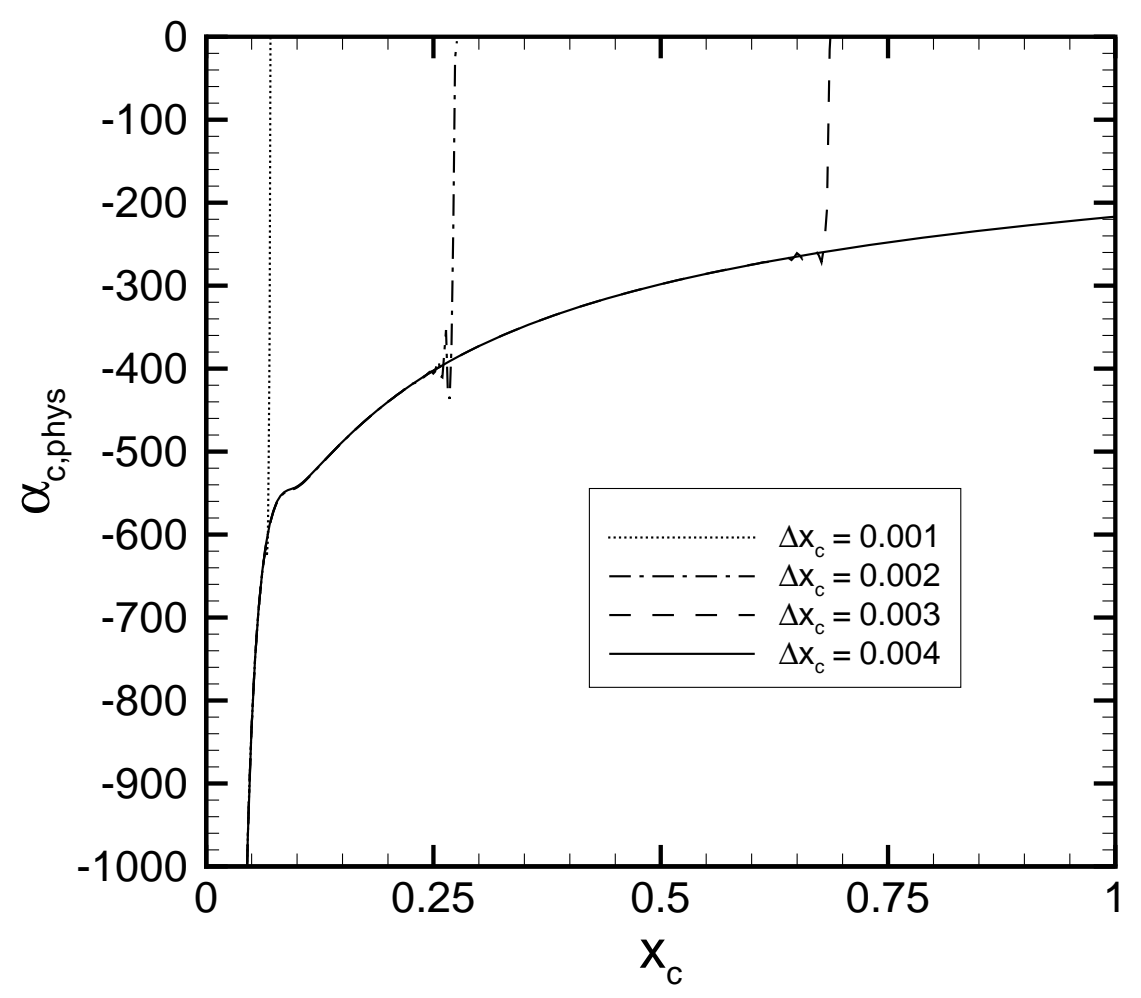

Figure 3.1: Physical $x_{c}$-wave number $\alpha_{c, \text { phys }}$ of crossflow vortex $\left(\beta_{c}=261.8\right)$ according to linear instability theory. With small step sizes in the PSE marching numerical instabilities occur and the solution blows up.

\subsection{Initial Conditions}

The initial conditions necessary to solve the PSE (3.22) as an initial-boundaryvalue problem can be specified in different ways. Both the choice of initial conditions for a given set of parameters $\left(\beta_{c}, \omega\right)$ and the $x_{c}$-location, where they are specified, influence the transients of the PSE-solution and hence the predicted amplitude evolution of disturbances. In order to keep the influence of possible transients in the physically interesting region, where the disturbance is amplified, as small as possible, it is advisable to start PSEcalculations well before the neutral point. However, since the growth rates of crossflow instabilities decrease very rapidly from the neutral point towards the attachment line, convergence problems restrict the choice of starting points for PSE-calculations .

One possibility to generate initial conditions for the PSE is to solve the classical local parallel instability equations (Orr-Sommerfeld-Squire formulation) at some starting point $x_{0}$. More consistent with the PSE formulation is the so-called local procedure developed by Bertolotti and Herbert (cf. Bertolotti [6]), which considers the wallnormal velocity component and the chordwise changes of the mean flow for the generation of initial conditions. In this approach, a Taylor-series expansion of $\hat{\mathbf{q}}$ and $\alpha_{c}$ around $x_{0}$ is introduced into 

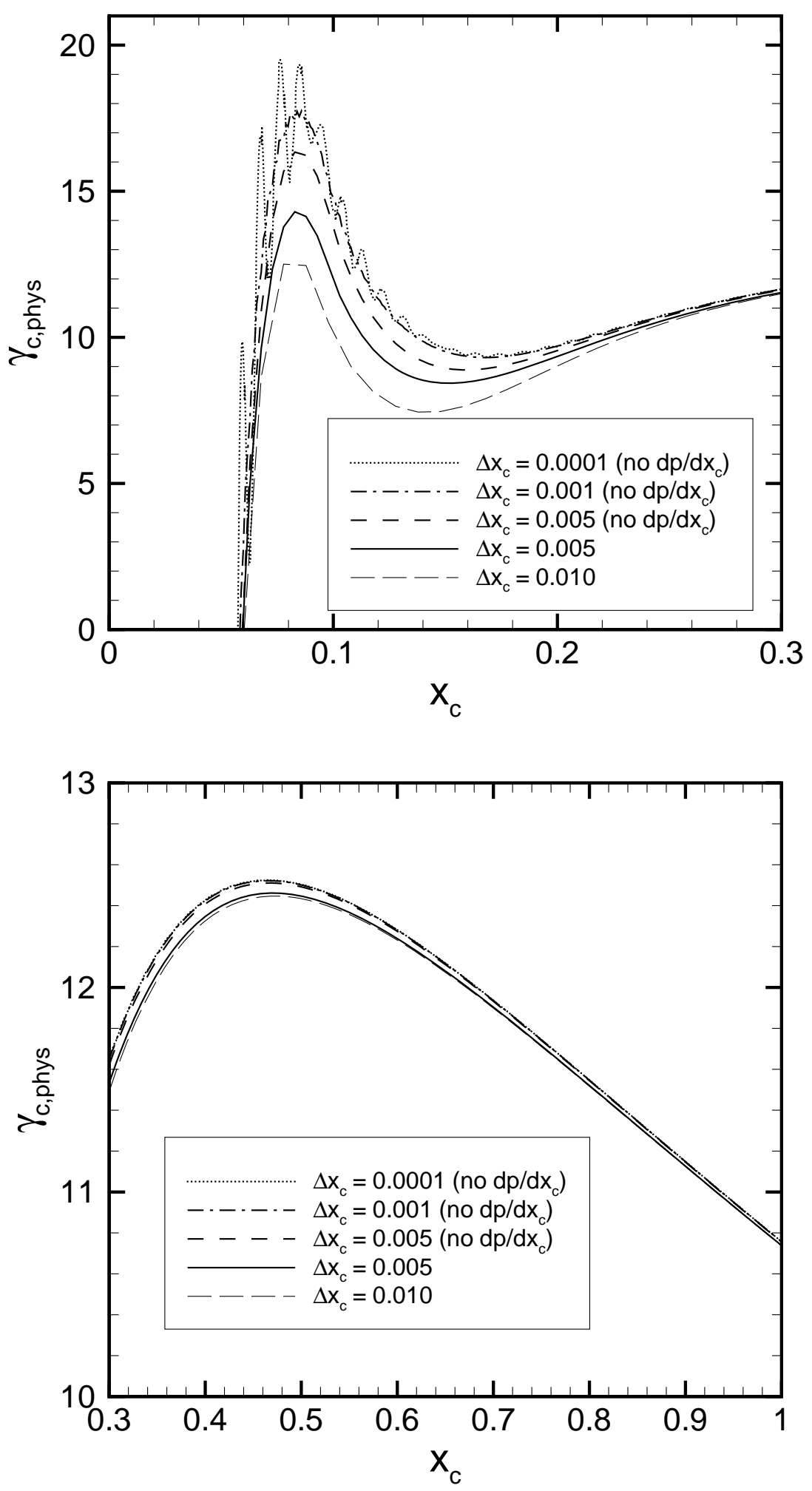

Figure 3.2: Growth rate $\gamma_{c, \text { phys }}$ of crossflow vortex $\left(\beta_{c}=261.8\right)$ for different step sizes with and without neglecting the $\partial \hat{p} / \partial x_{c}$-term in the PSE analysis (top: front part of the plate; bottom: rear part of the plate). 
the decomposition (3.15) - (3.17) of the disturbance function. Within the PSE-assumption, second and higher $x_{c}$-derivatives are neglected in the series, and the resulting equation is introduced into the auxiliary condition (3.28) and - together with a similar expansion of the base flow - into the PSE (3.22). Requiring the validity of the resulting equations both at $x_{0}$ and in the vicinity of $x_{0}$ leads to a system of equations for $\alpha_{c}\left(x_{0}\right), \partial \alpha_{c}\left(x_{0}\right) / \partial x_{c}, \hat{\mathbf{q}}\left(x_{c}, y\right)$, and $\partial \hat{\mathbf{q}}\left(x_{c}, y\right) / \partial x_{c}$. Together with homogeneous boundary conditions this system can be solved as an eigenvalue problem. As a mean between these two approaches one can keep the nonparallel base flow terms in the operator $L$ of the PSE (3.22), but neglect the chordwise changes of the disturbance flow (semi-nonparallel approach). A schematic comparison between these approaches is given in table 3.2 .

\begin{tabular}{||c||c|c|c||c|c|c||c|c|c|c||}
\hline \hline nonparallel & $U_{c}$ & $V$ & $W_{c}$ & $\frac{\partial U_{c}}{\partial x_{c}}$ & $\frac{\partial V}{\partial x_{c}}$ & $\frac{\partial W_{c}}{\partial x_{c}}$ & $\frac{\partial \tilde{c}_{c}}{\partial x_{c}}$ & $\frac{\partial \tilde{v}}{\partial x_{c}}$ & $\frac{\partial \tilde{w}_{c}}{\partial x_{c}}$ & $\frac{\partial \tilde{p}}{\partial x_{c}}$ \\
\hline semi-nonparallel & $U_{c}$ & $V$ & $W_{c}$ & $\frac{\partial U_{c}}{\partial x_{c}}$ & $\frac{\partial V}{\partial x_{c}}$ & $\frac{\partial W_{c}}{\partial x_{c}}$ & 0 & 0 & 0 & 0 \\
\hline parallel & $U_{c}$ & 0 & $W_{c}$ & 0 & 0 & 0 & 0 & 0 & 0 & 0 \\
\hline \hline
\end{tabular}

Table 3.2: Schematic comparison between nonparallel, semi-nonparallel, and parallel approach to generate initial conditions for the PSE.

For $\omega=0$ and $\beta_{c}=261.8$ spectra calculated using the semi-nonparallel approach are shown for different $x_{c}$-locations in figure 3.3. Circles mark the eigenvalues corresponding to the crossflow vortex considered in the previous section. It can be seen that this eigenvalue (and the whole spectrum) changes rather rapidly near the neutral point of the disturbance.

Figure 3.4 compares the growth rates $-\alpha_{c, i}$ of the crossflow vortex as predicted by the three approaches to generate initial conditions and shows that the nonparallel terms have a destabilizing effect. Disturbance profiles of this vortex calculated at $x_{c}=0.05$ using the three different approaches are compared in figure 3.5. The profiles are scaled such that the maximum of $\left|\hat{u}_{c}\right|$ is equal to one. In agreement with previous investigations of transonic boundary layers (cf. Stolte et al. [91]), the disturbance maximum is found to be closer to the wall if a nonparallel approach is used. For the $\hat{u}_{c}$ - and $\hat{w}_{c}{ }^{-}$ profiles nonparallel and semi-nonparallel results differ much less between each other than from parallel results. The wallnormal component of the disturbance velocity increases from the classical parallel prediction via seminonparallel theory to the local procedure.

Growth rates from PSE-calculations initialized with different initial conditions are compared in figure 3.6. The approach used to calculate initial conditions influences the position of the neutral point and disturbance amplification in a region of about $10 \%$ chord length. Previous investigations (see e.g. Simen et al. [88], Stolte et al. [91]) have shown a destabilizing effect of 

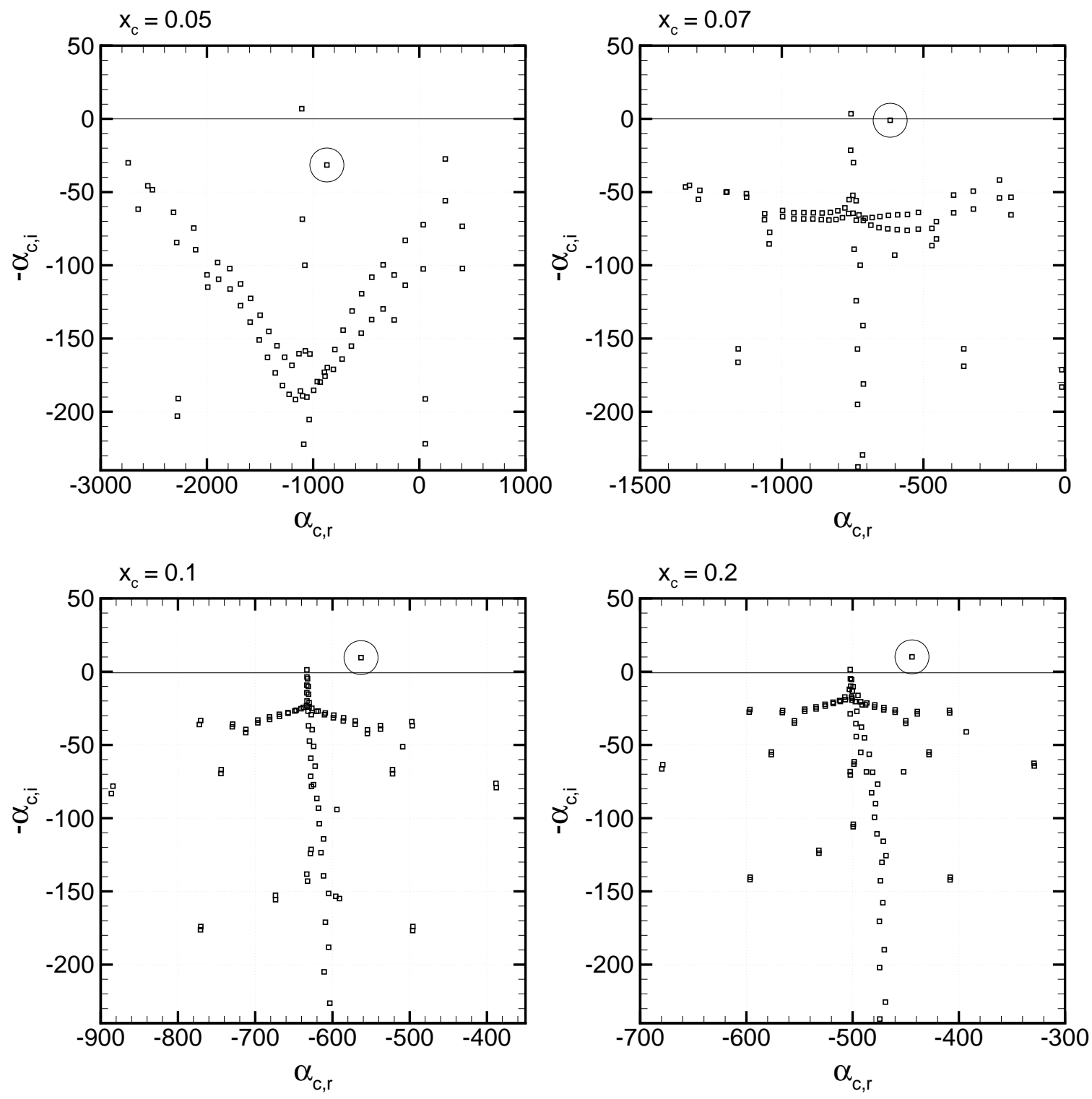

Figure 3.3: Spatial semi-nonparallel eigenvalue spectra for $\omega=0$ and $\beta_{c}=261.8$ at different $x_{c}$-locations. Circles mark the eigenvalues corresponding to the crossflow vortex. 


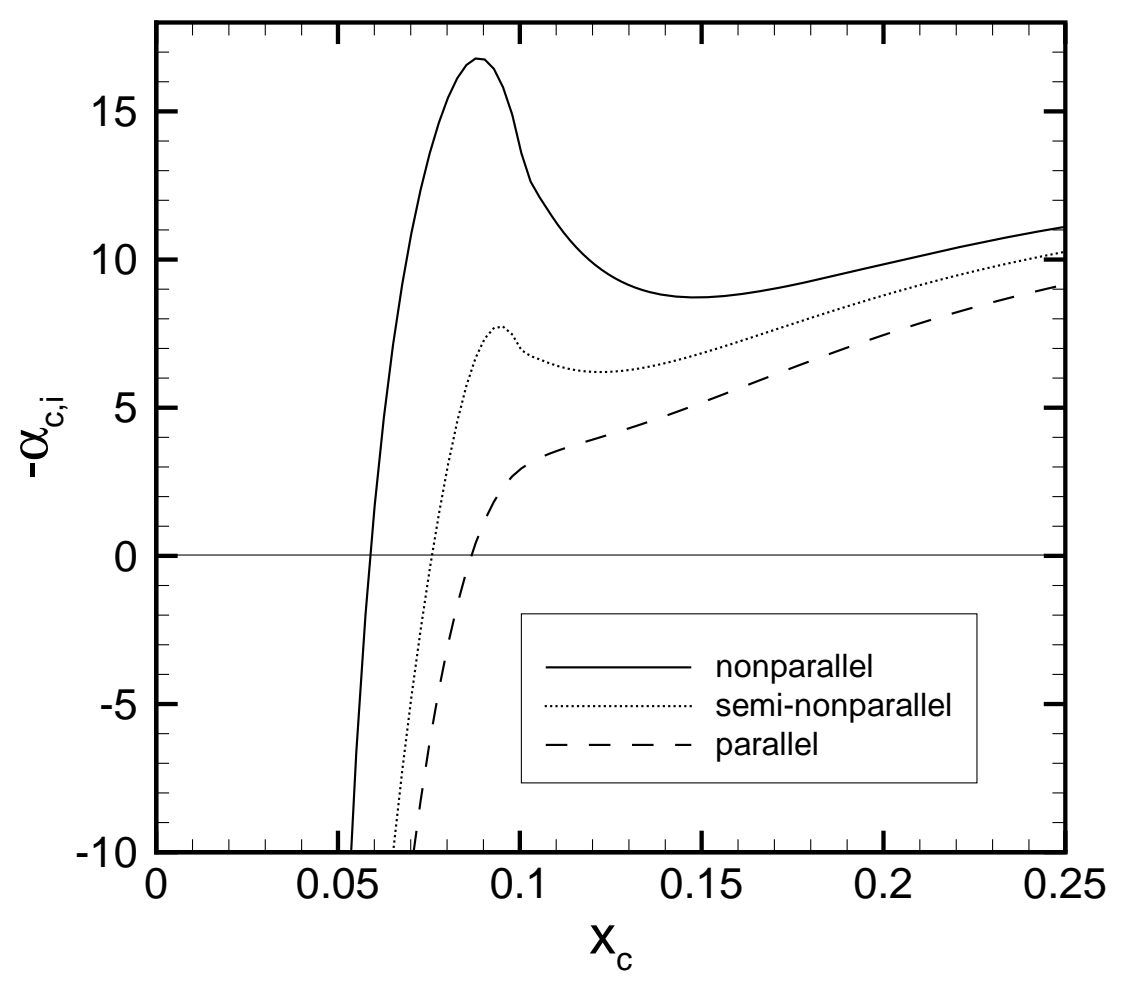

Figure 3.4: Growth rate $-\alpha_{c, i}$ of crossflow vortex $\left(\beta_{c}=261.8\right)$ as predicted by different approaches to generate initial conditions.
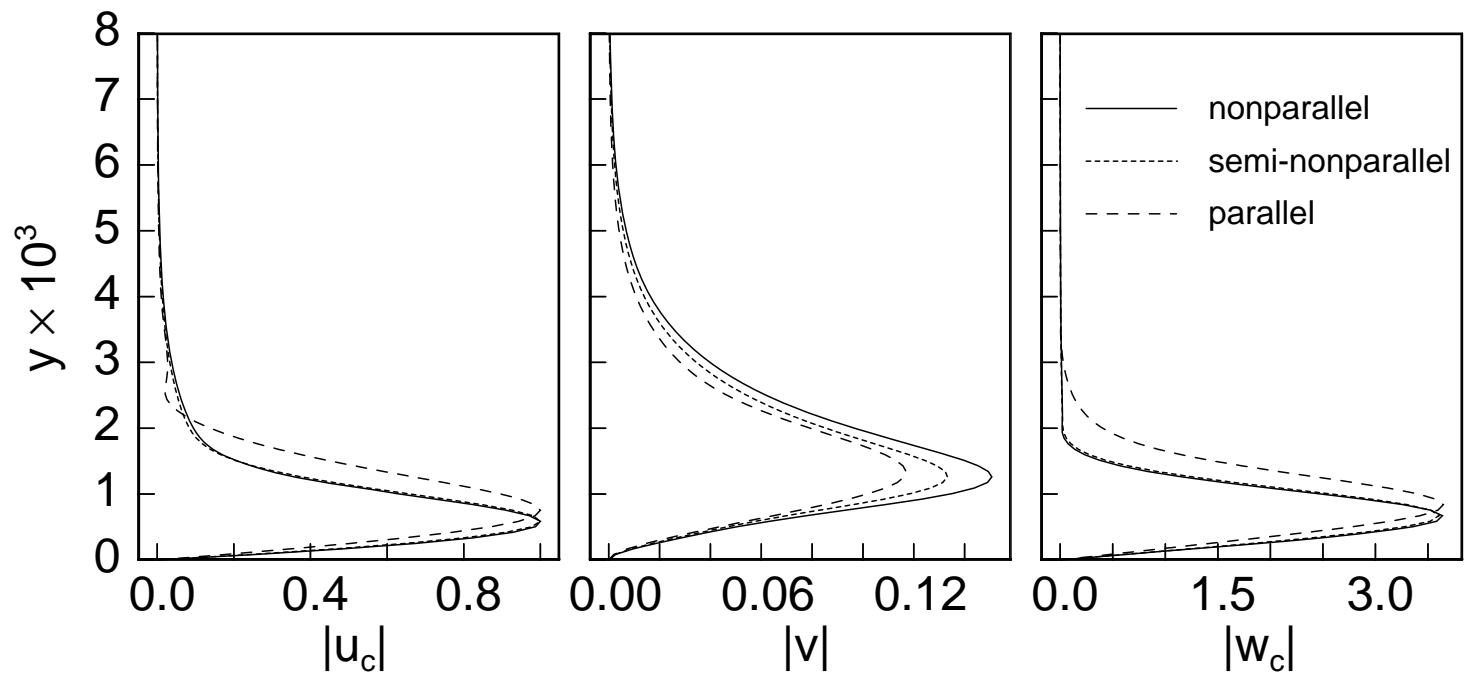

Figure 3.5: Eigenfunctions of disturbance velocity components as predicted by different approaches for a crossflow vortex with spanwise wave number $\beta_{c}=261.8$ at $x_{c}=0.05$. 
nonparallel and nonlocal terms in the instability equations. Figure 3.6 indicates that this effect is also present in an extended region of the flow, if the nonparallel and nonlocal terms are considered or neglected only in the equations used to calculate initial conditions, while the PSE remain unchanged. Most consistent with the PSE formulation are the nonparallel initial conditions, which will therefore be used in the following sections.

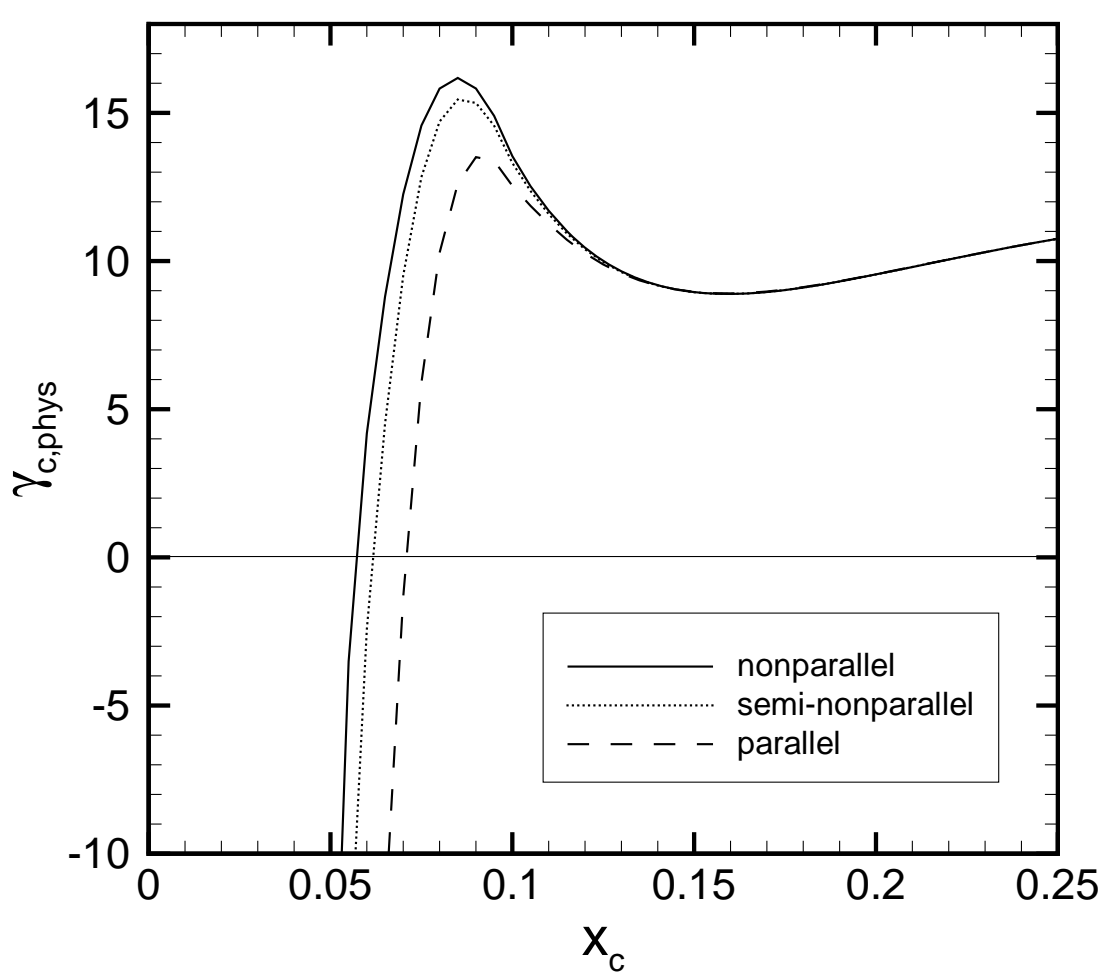

Figure 3.6: Growth rate $\gamma_{c, p h y s}$ of crossflow vortex $\left(\beta_{c}=261.8\right)$ with different initial conditions specified for the PSE at $x_{c}=0.05$.

In comparison to the differences due to different approaches to generate initial conditions, the choice of the chordwise location, where the PSEcalculation is started from, has rather small influence on disturbance amplification. Figure 3.7 shows this effect for nonparallel initial conditions specified at three different $x_{c}$-positions. Even though the third step of a calculation started at $x_{c}=0.05$ already gives a positive growth rate of the crossflow vortex, neutral point and growth rate do not change very much, if the PSE are initialized further upstream. However, initializing the PSE downstream of the neutral point leads to noticeable transients from the nonparallel initial conditions to the PSE results. If possible, initial conditions for the PSE should, hence, be specified at a chordwise location, where the disturbance of interest is still damped. 


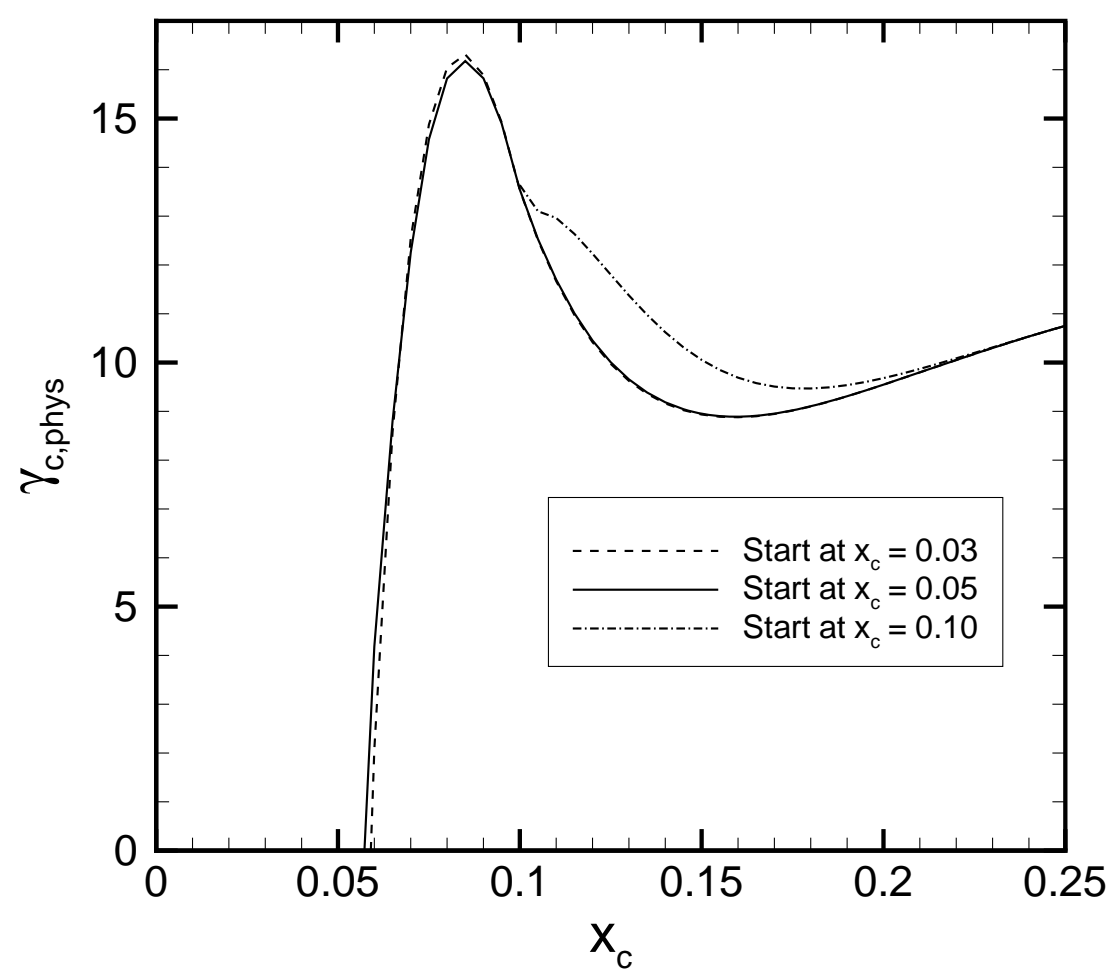

Figure 3.7: Growth rate $\gamma_{c, \text { phys }}$ of crossflow vortex $\left(\beta_{c}=261.8\right)$ with nonparallel initial conditions specified for the PSE at different chordwise positions.

\subsection{Parametric Investigations}

If the method of constant frequency and constant spanwise wave number as described in section 3.2 is used to calculate growth rates, the evolution of single disturbances is traced in a physically consistent way in the frame of linear nonlocal instability theory. However, it is not known a priori, which disturbances - i.e. disturbances of which frequency and spanwise wave number - exhibit the highest growth rates at different positions on the plate. Linear PSE-calculations for different parameters give an overview over the instability properties of the flow and are a prerequisite for the selection of disturbance modes for nonlinear instability analysis.

The model configuration of the DLR swept plate experiment has been investigated in detail for a variety of free stream conditions both experimentally and numerically. Bieler [9] showed that the experimentally observed (cf. Nitschke-Kowsky [69]) wave numbers of crossflow vortices and the frequencies of traveling disturbances agree with the parameters of strongly amplified modes according to temporal linear instability theory. His investigations, where the measured mean flow is approximated by a Falkner-SkanCooke flow, further confirmed that the spanwise wave length of steady vortices with strongest amplification is almost constant in chordwise direction. Janke [44] used a measured velocity distribution in the potential flow as 


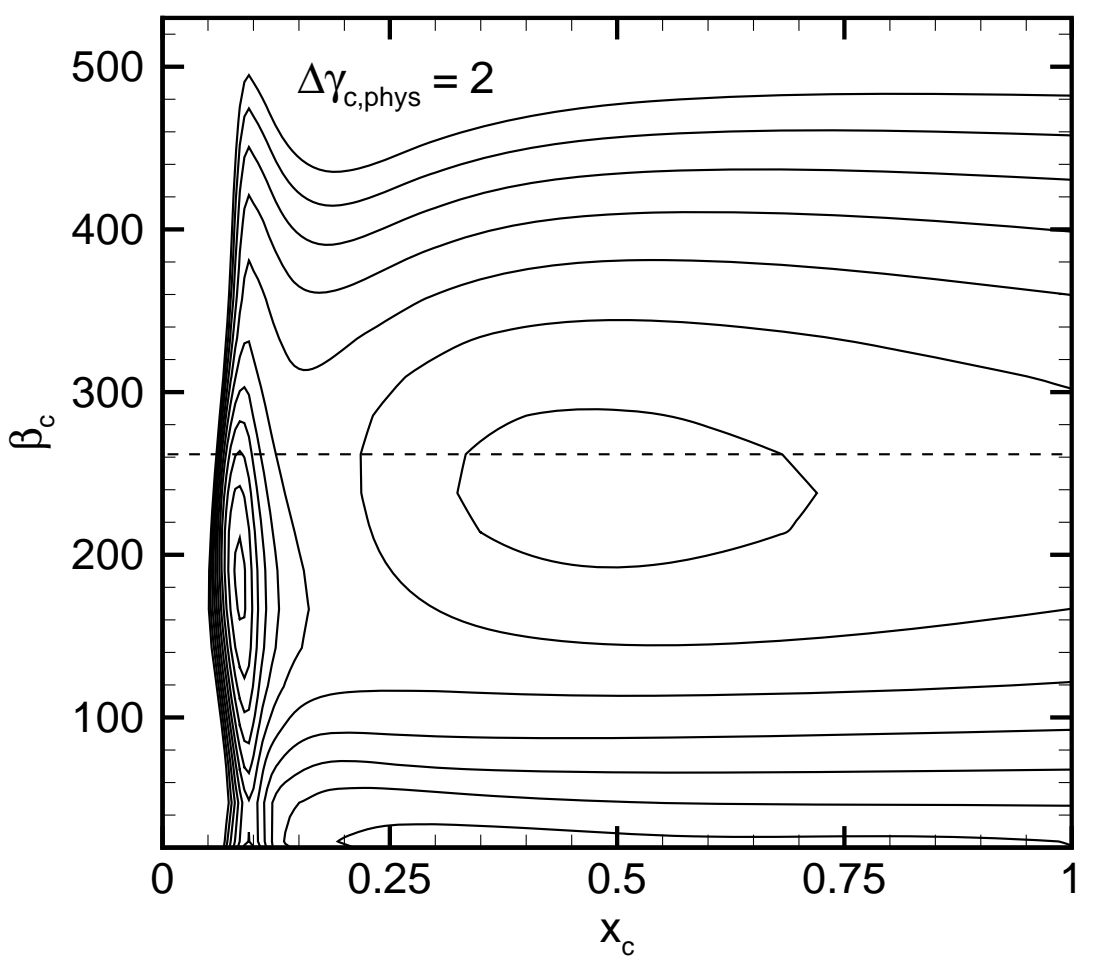

Figure 3.8: Growth rates $\gamma_{c, p h y s}$ of crossflow vortices $(f=0)$ in dependence of their spanwise wave number $\beta_{c}$. The outer contour line indicates the neutral curve $\left(\gamma_{c, \text { phys }}=0\right)$. The vortex with $\beta_{c}=261.8$ (marked by the dashed line) is used as fundamental primary disturbance in chapters 4 and 5 .

boundary condition for the calculation of a laminar base flow which he investigated using both spatial local instability theory and PSE computations. For free stream conditions similar to those specified in chapter 2, he found spanwise wave lengths $\lambda_{z, c}^{*}$ of vortices with strongest amplification according to spatial local instability theory varying between $10.43 \mathrm{~mm}\left(\beta_{c}=301.2\right)$ at $x_{c}=0.07$ and $13.32 \mathrm{~mm}\left(\beta_{c}=235.9\right)$ at $x_{c}=0.8$.

Growth rates of steady crossflow vortices are shown in figure 3.8 for PSE calculations with different spanwise wave numbers $\beta_{c}$. The peak between $x_{c}=0.05$ and $x_{c}=0.15$ must be interpreted with some care, since the growth rates predicted in this region are very sensitive to small changes in the pressure distribution. Downstream of $x_{c}=0.15$, the growth rates of disturbances do not change very much in chordwise direction. Vortices with spanwise wave numbers $\beta_{c}$ between 225 and 275 exhibit the highest growth rates downstream of $x_{c}=0.15$.

The evolution of crossflow vortices has been studied in detail for the free stream conditions specified in chapter 2 using nonlinear equilibrium solutions (cf. Koch et al. [53]) and spatial DNS (cf. Müller [67]). These investigations are guided by the measurements of Müller [65], who observed crossflow vortices with spanwise wave length $\lambda_{z, c}^{*}=12 \mathrm{~mm}$. As in Müller [67] 
and in Koch et al. [53] this disturbance will be considered as fundamental primary disturbance for the nonlinear PSE calculations in chapter 4.

Integral disturbance amplification as predicted by linear instability analysis is usually given in terms of $N$-factors

$$
N\left(x_{c}\right)=\ln \left(\frac{A\left(x_{c}\right)}{A_{0}}\right),
$$

where $A_{0}$ denotes the initial amplitude of the disturbance at the neutral point. It has to be kept in mind, however, that the initial disturbance amplitude $A_{0}$ does not enter the linear instability equations, which assume the disturbance to be infinitesimally small. Figure 3.9 (a) shows $N$-factors at the end of the test plate $\left(x_{c}=1.0\right)$ for crossflow vortices with different spanwise wave numbers. Maximal integral amplification can be observed for vortices with $\beta_{c} \approx 225$. This is partly due to the very strong amplification of vortices with spanwise wave numbers between $\beta_{c}=150$ and $\beta_{c}=250$ in the region near the attachment line.

Integral amplification of traveling disturbances with $\beta_{c}=261.8$ is shown in figure 3.9 (b), where the frequency is nondimensionalized by

$$
f=f^{*} \frac{c^{*}}{Q_{\infty}^{*}} .
$$

Here, positive frequencies denote waves traveling towards the leading edge, while negative frequencies denote waves traveling away from the leading edge. The "tail" for $f<-4$ is again due to disturbance amplification in the region near the attachment line. It can be seen that traveling crossflow modes with frequencies around $f=4$ experience significantly stronger amplification than steady crossflow vortices with the same spanwise wave number. Figure 3.10 summarizes the growth rates of these waves.

The angle between the propagation direction

$$
\psi_{c}=\arctan \left(\frac{\beta_{c}}{\alpha_{c, p h y s}}\right)
$$

of different crossflow modes with spanwise wave number $\beta_{c}=261.8$ and the orientation $\phi_{c}$ of the potential streamline with respect to the $x_{c}$-axis is shown in figure 3.11. Downstream of $x_{c}=0.2$ this relative orientation is almost constant in $x_{c}$-direction. The axis of the vortex $(f=0)$ is oriented at about $4^{\circ}$ from the potential streamline; with increasing frequency this angle increases to about $20^{\circ}$ for $f=6$ and even further for waves with higher frequencies (and rather low growth rates). 

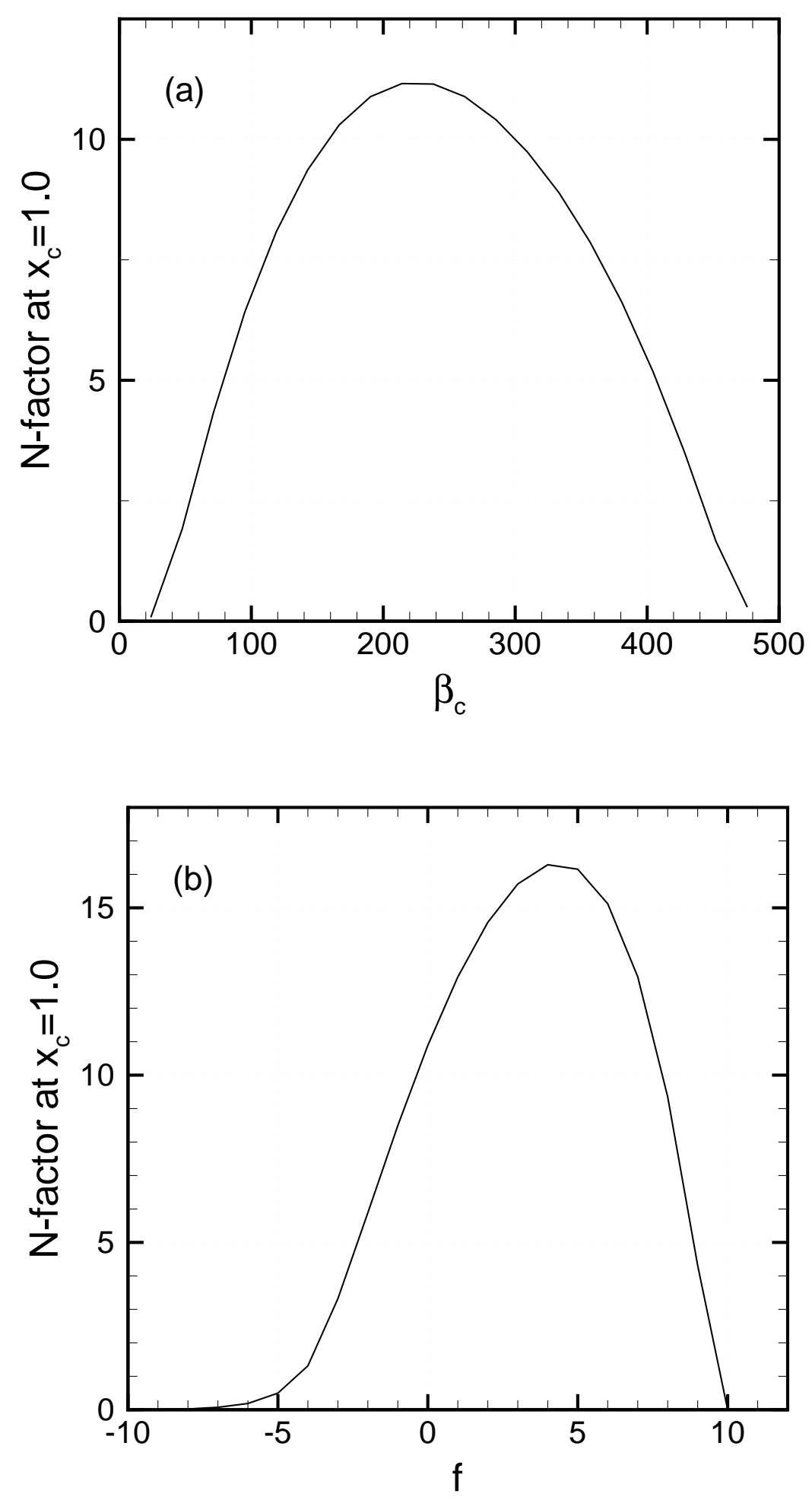

Figure 3.9: $N$-factors at $x_{c}=1.0$ from PSE calculations for crossflow vortices $(f=0)$ with different spanwise wave numbers $\beta_{c}$ (a) and for waves with different frequencies $f$ and spanwise wave number $\beta_{c}=261.8$ (b). 


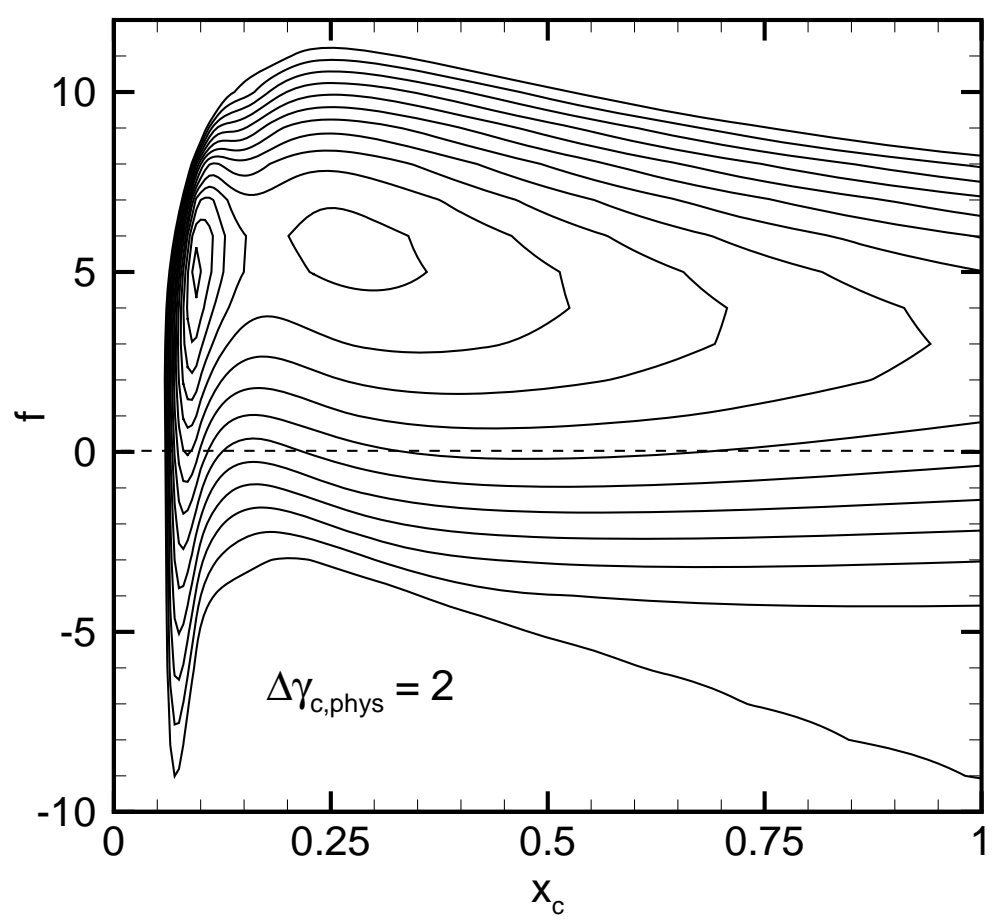

Figure 3.10: Growth rates $\gamma_{c, \text { phys }}$ of crossflow modes with spanwise wave number $\beta_{c}=261.8$ in dependence of their frequency $f$. The outer contour line indicates the neutral curve $\left(\gamma_{c, p h y s}=0\right)$. The steady vortex $(f=0)$ is marked by a dashed line.

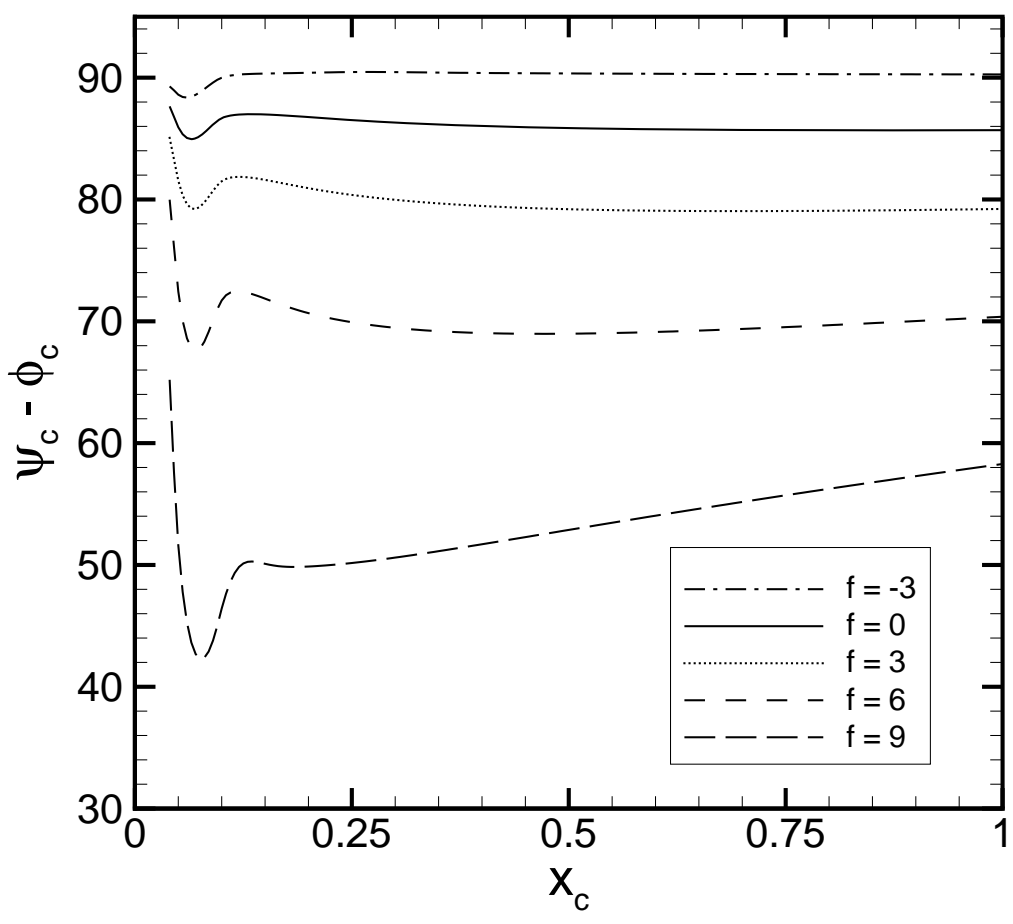

Figure 3.11: Angle between potential streamline and propagation direction $\psi_{c}$ of crossflow modes with spanwise wave number $\beta_{c}=261.8$ and different frequencies. 



\section{Chapter 4}

\section{Nonlinear Instability Analysis and Disturbance Interaction}

Interactions between disturbance modes, amplitude saturation, and meanflow distortion can only be captured by a nonlinear instability analysis. These features of nonlinear PSE computations are illustrated for the plane Poiseuille flow at $R e=6000$. For the base flow of the DLR swept plate experiment, stationary crossflow vortices, which can be expected to dominate the transition process in low-turbulence environments, are studied in detail. The spanwise wave length of the considered vortex is $12 \mathrm{~mm}$. By means of nonlinear interaction, the presence of the vortex generates additional inflection points in the distorted mean-flow profiles. The typical half-mushroom structure develops in the total flow field. In addition, small vortices very near to the wall are found in the distorted flow.

The presence of traveling crossflow waves (here, a wave with spanwise wave length $12 \mathrm{~mm}$ and frequency $135 \mathrm{~Hz}$ is considered) significantly reduces the amplification of stationary vortices, if the wave reaches high amplitudes earlier than the vortex. However, traveling waves do not strongly affect the evolution of stationary vortices, once the vortices have reached high amplitudes.

One central result from the nonlinear PSE analysis is that unique saturation amplitudes do neither exist for stationary crossflow vortices nor for traveling crossflow waves. This phenomenon is illustrated by artificially freezing the laminar base flow at various chordwise locations, and it is explained by means of a spatial bifurcation model. 


\subsection{Theoretical Background}

In the derivation of the linear PSE (3.22), product terms of disturbance quantities are neglected. As a consequence, the evolution of single disturbances can be studied independently for each mode. Interactions between different disturbances are not captured by any linear instability theory. Furthermore, linear theory is based on the concept of infinitesimally small disturbances. When the velocity fluctuations of disturbances reach the level of about $1 \%$ of the free stream velocity, the linear equations are no longer valid. A big advantage of the PSE is their ability to consistently incorporate nonlinear effects like amplitude saturation, nonlinear mean flow distortion and disturbance interaction. However, retaining the nonlinear terms (3.12) - (3.14) in the disturbance equations requires the consideration of some additional aspects of the PSE, which - taking pattern from Bertolotti [6] and Herbert [39] - are outlined in this section.

As in the linear formulation, the disturbance flow field $\tilde{\mathbf{q}}$ is assumed to be periodic in time $t$ and spanwise direction $z_{c}$, while the $x_{c}$-dependence is expanded in terms of slowly varying functions of $x_{c}$. For numerical studies only a finite number of modes in the resulting flow field can be considered and the physical disturbance flow field takes the form

$$
\tilde{\mathbf{q}}\left(x_{c}, y, z_{c}, t\right)=\sum_{l=-L}^{L} \sum_{n=-N}^{N} \sum_{k=-K}^{K} \hat{\mathbf{q}}_{(l, n, k)}\left(x_{c}, y\right) e^{i\left[\theta_{c,(l, n, k)}\left(x_{c}\right)+k \beta_{c}, 0 z_{c}-l \omega_{0} t\right]}
$$

where

$$
\frac{d \theta_{c,(l, n, k)}\left(x_{c}\right)}{d x_{c}}=\alpha_{c,(l, n, k)}\left(x_{c}\right)
$$

The choice of $\omega_{0}$ and $\beta_{c, 0}$ corresponds to choosing the computational domain in a Navier-Stokes simulation. The physical solutions of the PSE have to be real. Thus,

$$
\hat{\mathbf{q}}_{(-l,-n,-k)}\left(x_{c}, y\right)=\hat{\mathbf{q}}_{(l, n, k)}^{\dagger}\left(x_{c}, y\right), \quad \alpha_{c,(-l,-n,-k)}\left(x_{c}\right)=\alpha_{c,(l, n, k)}^{\dagger}\left(x_{c}\right) .
$$

Disturbance amplification and chordwise wave number $\alpha_{c}$ depend on the chordwise coordinate $x_{c}$ as in the linear ansatz (3.15) - (3.17). In addition, formulation (4.1) - (4.2) allows for the existence of several modes with the same spanwise wave number $\beta_{c}$, angular frequency $\omega$ and different chordwise wave number $\alpha_{c}$, which thus have different phase velocity $c_{0}=\omega / \alpha_{c, r}$. This additional degree of freedom is connected with a difficulty in the analysis of disturbance interaction. A single disturbance mode takes the form

$$
\tilde{\mathbf{q}}_{(l, n, k)}\left(x_{c}, y, z_{c}, t\right)=\hat{\mathbf{q}}_{(l, n, k)}\left(x_{c}, y\right) e^{i\left[\theta_{c,(l, n, k)}\left(x_{c}\right)+k \beta_{c, 0} z_{c}-l \omega_{0} t\right]}+\text { c.c. }
$$

and can be denoted by an index vector

$$
\mathbf{p}=(l, n, k),
$$


where $\mathbf{p}$ and $-\mathbf{p}$ refer to the same physical disturbance. The interaction of two modes $\mathbf{p}_{\mathbf{1}}$ and $\mathbf{p}_{\mathbf{2}}$ generates product terms with indices $\mathbf{p}_{\mathbf{1}}+\mathbf{p}_{\mathbf{2}}$ and $\mathbf{p}_{\mathbf{1}}-\mathbf{p}_{\mathbf{2}}$. Unless they are neglected, those terms have to be compensated by disturbance modes with corresponding wave numbers and frequencies. If such a disturbance is not present in the considered flow field, it is generated and added to the spectrum of disturbance modes.

As far as spanwise wave number $\beta_{c}$ and angular frequency $\omega$ are concerned, the relevant procedure is obvious, since these quantities are constants and can take on only values that are integer multiples of $\beta_{c, 0}$ and $\omega_{0}$. If these values are inside the discretized spectra $-K \beta_{c, 0}, \ldots, K \beta_{c, 0}$ and $-L \omega_{0}, \ldots, L \omega_{0}$, respectively, the corresponding terms can be ranged in the group of considered disturbances, otherwise they are neglected. The complex exponent $\alpha_{c,}$ however, is unknown a priori and depends on $x_{c}$. Hence, the chordwise wave number $\alpha_{c, r}$ can not be integrated into a discrete wave number spectrum without further ado. A discretization of the chordwise wave number spectrum and, hence, a compensation of the above mentioned product terms by suitable disturbance modes can be obtained by employing a modified form of the auxiliary condition (3.28) for every disturbance mode.

In order to derive this condition, let $\alpha_{c, r, 0}$ be the smallest positive chordwise wave number to be resolved by the PSE calculation. The product term with indices $l=l_{0}$ and $k=k_{0}$ is then expanded into a series of exponential functions in $x_{c}$ with exponents $i \alpha_{c,\left(l_{0}, n, k_{0}\right)}, n \in \mathcal{Z}$. The real parts of $\alpha_{c,\left(l_{0}, n, k_{0}\right)}, n \in \mathcal{Z}$, are required to fall into intervals with length $2 d$ around integer multiples of $\alpha_{c, r, 0}$ :

$$
\left|\alpha_{c, r,\left(l_{0}, n, k_{0}\right)}-n \alpha_{c, r, 0}\right|<d \quad \forall n \in\{-N, \ldots, N\} .
$$

Thus, if a disturbance $\left(l_{1}, n_{1}, k_{1}\right)$ interacts with a disturbance $\left(l_{2}, n_{2}, k_{2}\right)$, two classes of product terms with chordwise wave numbers $\alpha_{c, r,\left(l_{1}+l_{2}, n_{1}+n_{2}, k_{1}+k_{2}\right)}$ and $\alpha_{c, r,\left(l_{1}-l_{2}, n_{1}-n_{2}, k_{1}-k_{2}\right)}$ are generated. These wave numbers satisfy the conditions

$$
\left|\alpha_{c, r,\left(l_{1}+l_{2}, n_{1}+n_{2}, k_{1}+k_{2}\right)}-\left(n_{1}+n_{2}\right) \alpha_{c, r, 0}\right|<2 d,
$$

and

$$
\left|\alpha_{c, r,\left(l_{1}-l_{2}, n_{1}-n_{2}, k_{1}-k_{2}\right)}-\left(n_{1}-n_{2}\right) \alpha_{c, r, 0}\right|<2 d,
$$

respectively. Hence, in the set of possible disturbances with respect to the specific discretization, for each product term exists a disturbance $\mathbf{p}$ with a spanwise wave number $\alpha_{c, r, \mathbf{p}}$ differing less than $d$ from $\left(n_{1}+n_{2}\right) \alpha_{c, r, 0}$ or $\left(n_{1}-\right.$ $\left.n_{2}\right) \alpha_{c, r, 0}$ and, consequently, less than $3 d$ from the chordwise wave number of the corresponding product term. If wave numbers and angular frequency of $\mathbf{p}$ are within the bounds of the corresponding spectra, $\mathbf{p}$ can compensate the contribution of the product term in the nonlinear PSE. The additional slow $x_{c}$-variation due to the difference between $\alpha_{c, r, \mathbf{p}}$ and $\alpha_{c, r, \mathbf{p}_{\mathbf{1}}+\mathbf{p}_{2}}$ or $\alpha_{c, r, \mathbf{p}_{1}-\mathbf{p}_{\mathbf{2}}}$ is transferred to the amplitude function $\hat{u}_{\mathbf{p}}$ by means of a generalized auxiliary condition

$$
\int_{0}^{y_{e}} \hat{u}_{c, \mathbf{p}}^{\dagger}\left\{\frac{\partial \hat{u}_{c, \mathbf{p}}}{\partial x_{c}}-f\left(\alpha_{c, r, \mathbf{p}}\right) \hat{u}_{c, \mathbf{p}}\right\} d y=0,
$$


where

$$
f\left(\alpha_{c, r, \mathbf{p}}\right)=\sigma\left(\alpha_{c, r, \mathbf{p}}-n \alpha_{c, r, 0}\right) .
$$

For sufficiently high values of the weight $\sigma$, the chordwise wave number $\alpha_{c, r, \mathbf{p}}$ is constrained to stay in the vicinity of $n \alpha_{c, r, 0}$. This leads to an increasing $x_{c}$-variation of the amplitude function $\hat{\mathbf{q}}_{\mathbf{p}}$ and, hence, of the error due to the PSE approximation. Often, the chordwise wave numbers of disturbances naturally are integer multiples of each other. In this case, small values of $\sigma$ (e.g. $\sigma \leq 0.1$ ) are appropriate. For $\sigma=0$, the auxiliary condition (4.9) passes into form (3.28).

Introducing ansatz (4.1) - (4.2) into the nonlinear disturbance equations (3.8) - (3.11) and applying the PSE approximation yields the nonlinear PSE

$$
L_{\mathbf{p}} \hat{\mathbf{q}}_{\mathbf{p}}+M_{\mathbf{p}} \frac{\partial \hat{\mathbf{q}}_{\mathbf{p}}}{\partial x_{c}}+\frac{d \alpha_{c, \mathbf{p}}}{d x_{c}} N_{\mathbf{p}} \hat{\mathbf{q}}_{\mathbf{p}}=\hat{\mathbf{r}}_{\mathbf{p}}
$$

where - as in (3.22) - the operators $L_{\mathbf{p}}, M_{\mathbf{p}}$, and $N_{\mathbf{p}}$ act only in y. The PSE 4.11 are solved together with the auxiliary condition 4.9. The right-hand side $\hat{\mathbf{r}}_{\mathbf{p}}=\hat{\mathbf{r}}_{\mathbf{p}}\left(x_{c}, y\right)$ is the sum over all terms contributing to mode $\mathbf{p}$ :

$$
\hat{\mathbf{r}}_{\mathbf{p}}=\sum_{\mathbf{s} \in[-L, L] \times[-N, N] \times[-K, K]} R_{\mathbf{p}}\left(\hat{\mathbf{q}}_{\mathbf{s}}, \hat{\mathbf{q}}_{\mathbf{p}-\mathbf{s}}\right) e^{i \int_{x_{c, 0}}^{x_{c}}\left(\alpha_{c, \mathbf{s}}+\alpha_{c, \mathbf{p}-\mathbf{s}}-\alpha_{c, \mathbf{p}}\right) d x_{c}^{\prime}} .
$$

Initial conditions for each disturbance are generated as in linear studies. Usually, only a small number of disturbances is initialized at $x_{0}$. Due to nonlinear forcing, additional modes are generated in the course of the computation. Together with appropriate boundary conditions equations (4.11) and (4.9) can be integrated downstream. In the iterative solution at each $x_{c}$-position, the evaluation of the right-hand side $\hat{\mathbf{r}}_{\mathbf{p}}$ is retarded by one iterate in the Newton procedure, so that $\hat{\mathbf{r}}_{\mathbf{p}}$ contains no unknowns. As in linear PSE analysis, homogeneous boundary conditions (3.23) at the wall and at the boundary layer edge can be specified for all modes except the nonlinear mean flow distortion $(\mathbf{p}=(0,0,0))$. The wallnormal velocity component $v$ of this mode does not go to zero at the boundary layer edge due to the changes in displacement thickness of the boundary layer caused by the growth of disturbances. Thus,

$$
y \rightarrow \infty: \quad \frac{\partial \hat{v}_{(0,0,0)}}{\partial y} \rightarrow 0
$$

is the appropriate boundary condition for the mean flow distortion. 


\subsection{Nonlinear Growth in a Parallel Base Flow}

In order to illustrate main features of nonlinear PSE calculations and to set a starting point for the discussion of nonlinear amplitude saturation, a parallel base flow is investigated in this section.

As a prototype for shear flows and to illustrate fundamentals of theories, the plane Poiseuille flow has been examined by many investors because it is an exact solution of the Navier-Stokes equations with a finite critical Reynolds number. Details of linear and nonlinear instability properties of this flow are given in the textbook by Drazin \& Reid [19]. The plane Poiseuille flow, an incompressible flow between two parallel plates at rest as depicted in figure 4.1 satisfies the momentum equation

$$
\nu^{*} \frac{d^{2} U^{*}\left(y^{*}\right)}{d y^{* 2}}=\frac{1}{\rho^{*}} \frac{d P^{*}\left(x^{*}\right)}{d x^{*}} .
$$

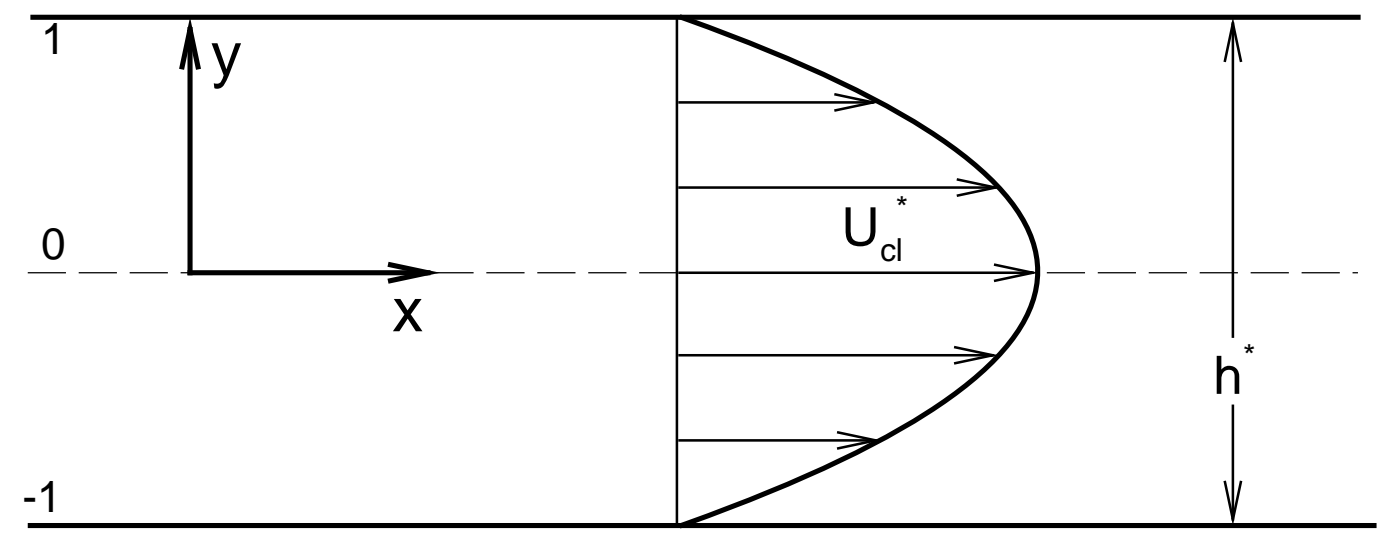

Figure 4.1: Plane Poiseuille flow. Laminar base flow and coordinate system.

In nondimensional form and together with homogeneous boundary conditions at the plates

$$
U(-1)=U(1)=0
$$

the laminar base flow can be written as

$$
U(y)=1-y^{2} ; \quad \frac{d P}{d x}=\text { const. },
$$

where the reference length $L^{*}=h^{*} / 2$ is half the channel width and the reference velocity $U_{c l}^{*}$ is the velocity at the center of the channel. Defining the Reynolds number as

$$
R e=\frac{U_{c l}^{*} L^{*}}{\nu^{*}}
$$

the linear and nonlinear instability properties of this flow can be examined. A numerical study of the corresponding Orr-Sommerfeld equation has 

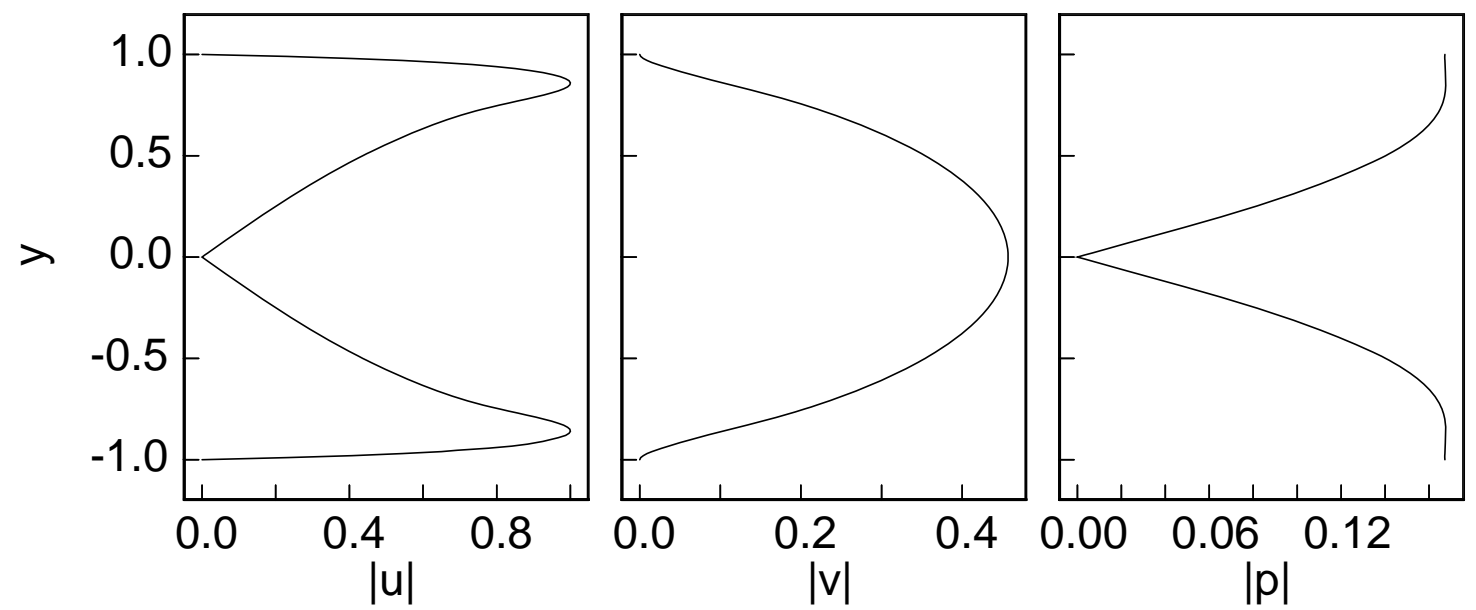

Figure 4.2: Modulus of disturbance profiles of wave with $\omega=0.2598$ and $\alpha_{r}=1$ in plane Poiseuille flow at $R e=6000$ (spatial linear theory).

been performed by Orszag [72], who obtained a critical Reynolds number $R e_{\text {crit }}=5772.22$. The disturbance with neutral stability at $R e_{\text {crit }}$ has a streamwise wave number $\alpha_{\text {crit }}=1.02056$ and a phase velocity $c=\omega / \alpha_{\text {crit }}=0.264002$. As starting point for the nonlinear calculations, the unstable eigenmode has been computed for the supercritical Reynolds number $R e=6000$ and the streamwise wave number $\alpha=1.0$. This mode has the temporal growth rate $\omega_{i}=3.231 \cdot 10^{-4}$ and the angular frequency $\omega_{r}=0.2598$.

According to spatial theory, this mode with $\omega=0.2598$ has a growth rate $-\alpha_{i}=8.526 \cdot 10^{-4}$. The disturbance profiles of this wave, which is denoted by $(1,1,0)$ according to the nomenclature introduced in section 4.1 , are shown in figure 4.2. If this mode is initialized at some low amplitude and traced downstream using the nonlinear PSE, its amplitude first increases with a growth rate similar to that predicted by linear theory. The forcing due to the nonlinear terms results in a nonlinear distortion of the mean flow absorbed into mode $(0,0,0)$ and the generation of higher modes $(n, n, 0)$. Due to its nonlinear deformation the stability properties of the mean flow change as can be seen from figure 4.3. In comparison with the amplification predicted by linear theory the growth rate of mode $(1,1,0)$ first increases slightly. Further downstream all disturbances including the mean flow distortion stop growing; a saturated state is attained. The nonlinear deformation of the mean flow in the saturated state is illustrated in figure 4.4. As can be seen from figure 4.5, the saturation amplitude does not depend on the initial amplitude of the disturbance. If the wave is initialized at higher amplitude, the same nonlinear saturated state is achieved. 


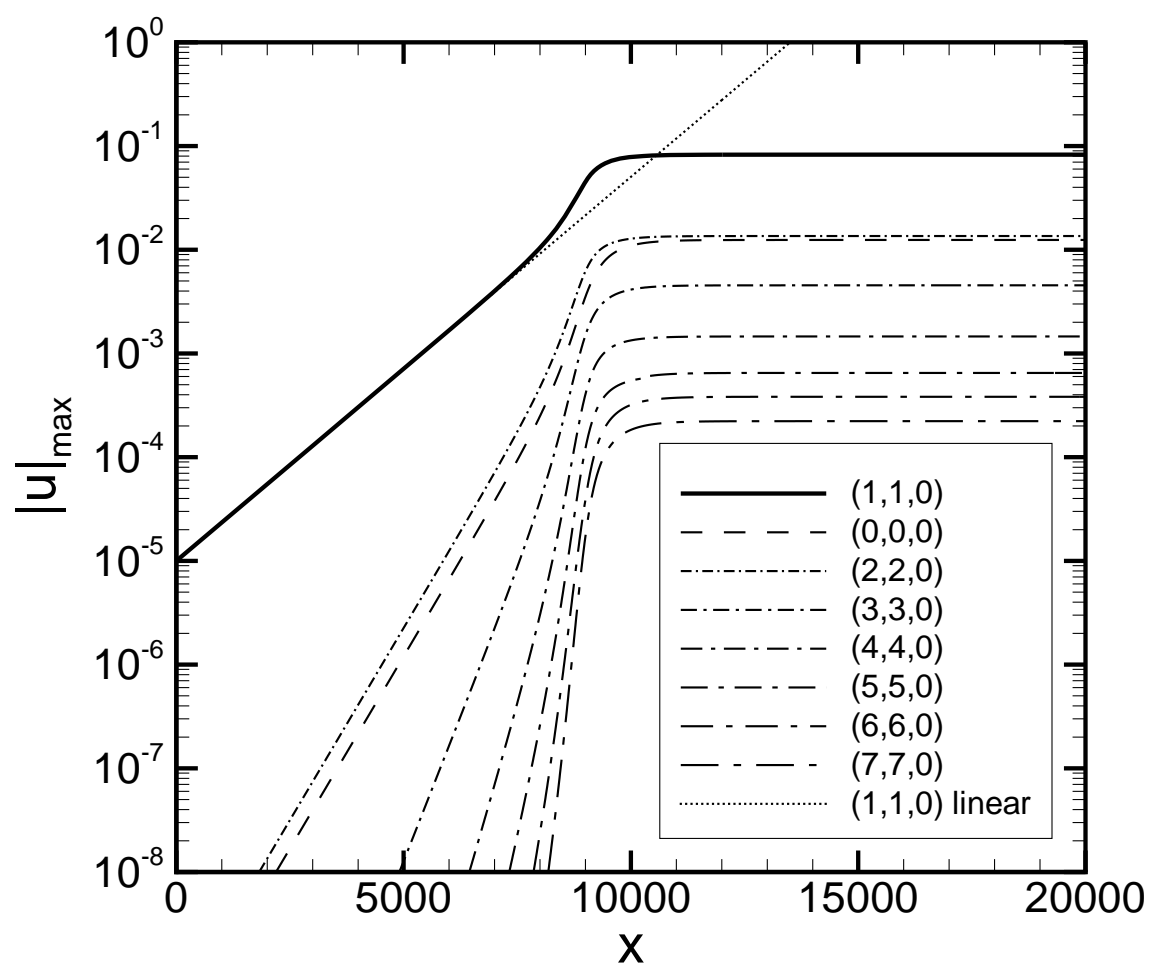

Figure 4.3: Nonlinear amplification of disturbance with $\omega=0.2598$ in plane Poiseuille flow at $R e=6000$.

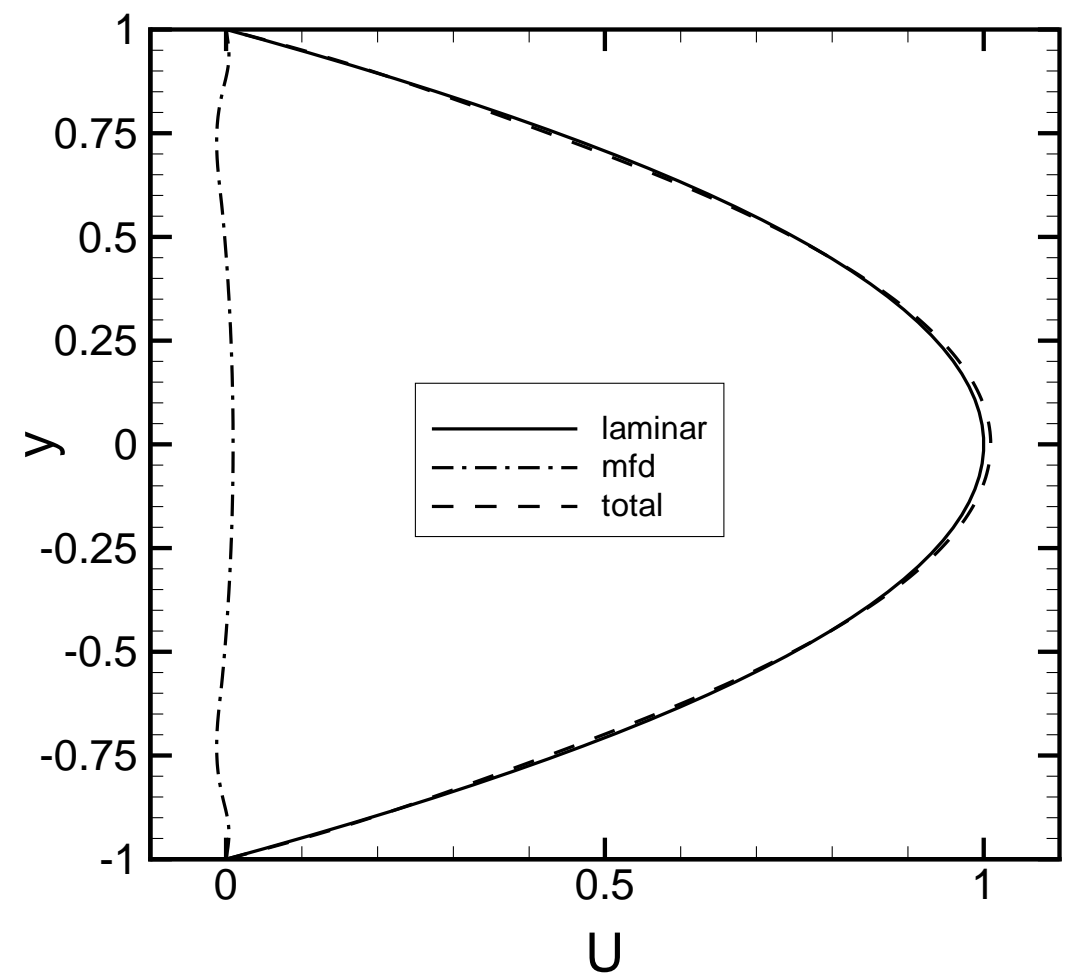

Figure 4.4: Distorted mean flow profile in the saturated nonlinear state. 


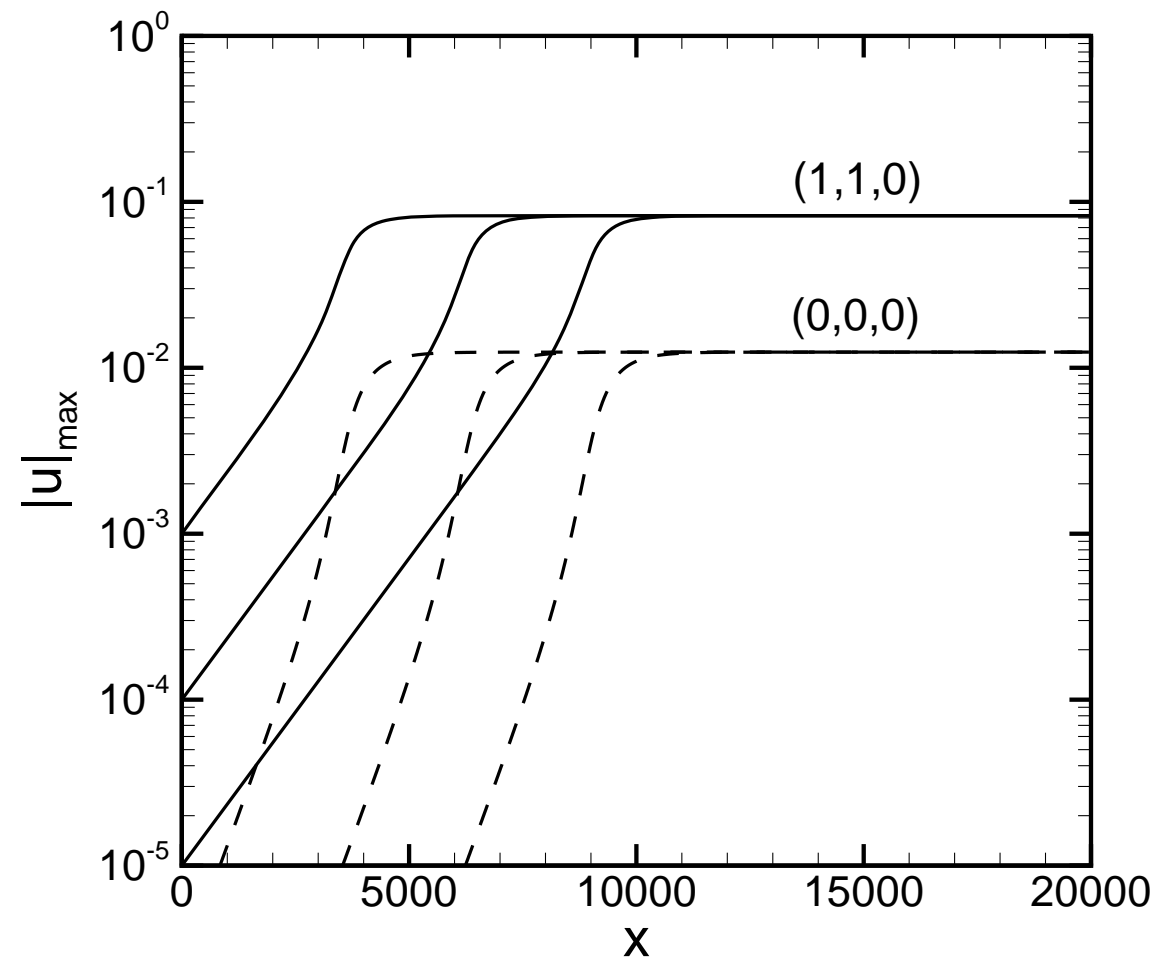

Figure 4.5: Nonlinear amplification of disturbance with $\omega=0.2598$ and various initial amplitudes in plane Poiseuille flow at $R e=6000$. Modes up to $(7,7,0)$ are considered in the computation.

\subsection{Nonlinear Amplification of Cross-Flow Vortices}

According to linear instability theory, traveling crossflow modes have higher growth rates than stationary vortices (see section 3.5). While vortices are generated by surface roughness, traveling waves are produced by the interaction of free stream turbulence with the boundary layer. In spite of their lower growth rates crossflow vortices can therefore be expected to dominate the transition process in low-turbulence environments as found in free flight. For that reason, the nonlinear evolution of a crossflow vortex with spanwise wave length $\lambda_{z, c}^{*}=12 \mathrm{~mm}\left(\beta_{c}=261.8\right)$ without any traveling disturbances present in the flow field is investigated in this section. The interaction of stationary and traveling crossflow modes is studied in section 4.4. Unless explicitly mentioned, the disturbance amplitudes are presented in terms of the maximum of the disturbance velocity component $\left|\tilde{u}_{c}\right|$ in $x_{c}$-direction (not in direction of the potential streamline). For stationary vortices, the maximum is determined both in $x_{c}$ - and $z_{c}$-direction. For the spectral collocation method in $y$-direction, four sub-domains with 15 Chebyshev polynomials are used in the computations considering up to four harmonics of the fundamental mode. If more harmonics are considered, seven sub-domains with 15 polynomials are used. For the comparison between the amplitudes 
presented by different authors, it should be mentioned, that the total free stream velocity $Q_{\infty}^{*}=19 \mathrm{~m} / \mathrm{s}$ is used as reference velocity in this work, while B. Müller [65] and W. Müller [67] use the component normal to the leading edge of the plate $U_{\infty}^{*}=14 \mathrm{~m} / \mathrm{s}$ and Koch et al. [53] use the local reference velocity $Q_{e}^{*}=Q_{\infty}^{*}\left[\sin ^{2} \phi_{\infty}+\left(1-c_{p}\right) \cos ^{2} \phi_{\infty}\right]^{1 / 2}$.

For the PSE computations presented in this section only the mode $(0,-1,1)$ is initialized by a solution of the linear nonparallel local procedure (see section $3.4)$ at $x_{c}=0.04$. The nonlinear mean flow distortion $(0,0,0)$ and the higher modes $(0,-n, n)$ are generated in the course of the downstream marching by forcing due to the nonlinear terms. Receptivity, i.e. the generation of vortices by surface roughness is not considered in the formulation. Thus, the initial disturbance amplitude, which plays an important role in the transition process, is an additional parameter. Since the disturbance is initialized upstream of the neutral point, the amplitude at the neutral point is given as initial disturbance amplitude. This amplitude is $\left|\tilde{u}_{c}\right|_{\max }=7.2 \cdot 10^{-5}$ in the computation presented in figure 4.6. In the first half of the plate the amplification of the vortex $(0,-1,1)$ agrees with that predicted by linear theory. Forced by nonlinear terms, the mean flow distortion $(0,0,0)$ and the harmonic $(0,-2,2)$ grow with the fundamental vortex. The amplitudes of the forced modes $(0,0,0)$ and $(0,-2,2)$ are of the order of the square of the amplitude of the forcing mode $(0,-1,1)$. From figure 3.8 can be seen that the harmonics of mode $(0,-1,1)$ are all stable according to linear instability theory. At $x_{c}=0.58$, the fundamental vortex $(0,-1,1)$ has reached an amplitude of $3 \%$ and starts to deviate from the linear prediction. Nonlinear saturation sets in. The amplitude of the vortex reaches its maximum of $23 \%$ at $x_{c}=0.95$. There, the mean flow distortion has an amplitude of $14.5 \%$, and mode $(0,-2,2)$ has an amplitude of $11.5 \%$. Further downstream, the amplitude of mode $(0,-1,1)$ (in spite of large positive growth rates according to linear instability theory) even decays slightly to $22.5 \%$ at $x_{c}=1.0$, where the mean flow distortion has an amplitude of $17.2 \%$.

If the vortex $(0,-1,1)$ is initialized with a ten times higher amplitude of 7.2 . $10^{-4}$ at the neutral point $x_{c}=0.06$, it exceeds $3 \%$ amplitude already at $x_{c}=0.4$ and consequently deviates earlier from the linear prediction as can be seen in figure 4.7. It reaches an amplitude of $21 \%$ at $x_{c}=0.8$, where the mean flow distortion has an amplitude of $12.2 \%$, and continues to grow slowly up to an amplitude of $22 \%$ at $x_{c}=1.0$. There, the mean flow distortion has an amplitude of $12.8 \%$ - significantly lower than in the case, where the vortex $(0,-1,1)$ is initialized with an amplitude of $7.2 \cdot 10^{-5}$. Even though the amplitude of the fundamental vortex $(0,-1,1)$ increases monotonically downstream of the neutral point if the mode is initialized with amplitude $7.2 \cdot 10^{-4}$, the higher modes $(0,-3,3)$ and $(0,-4,4)$ do not reach their maximal amplitude at the end of the plate. Mode $(0,-3,3)$ reaches an amplitude of $6.3 \%$ at $x_{c}=0.88$ and then decays to $5.6 \%$ at $x_{c}=1.0$, mode $(0,-4,4)$ reaches its maximum of $4.3 \%$ at $x_{c}=0.84$, before it decays to $3.5 \%$ at the end of the 


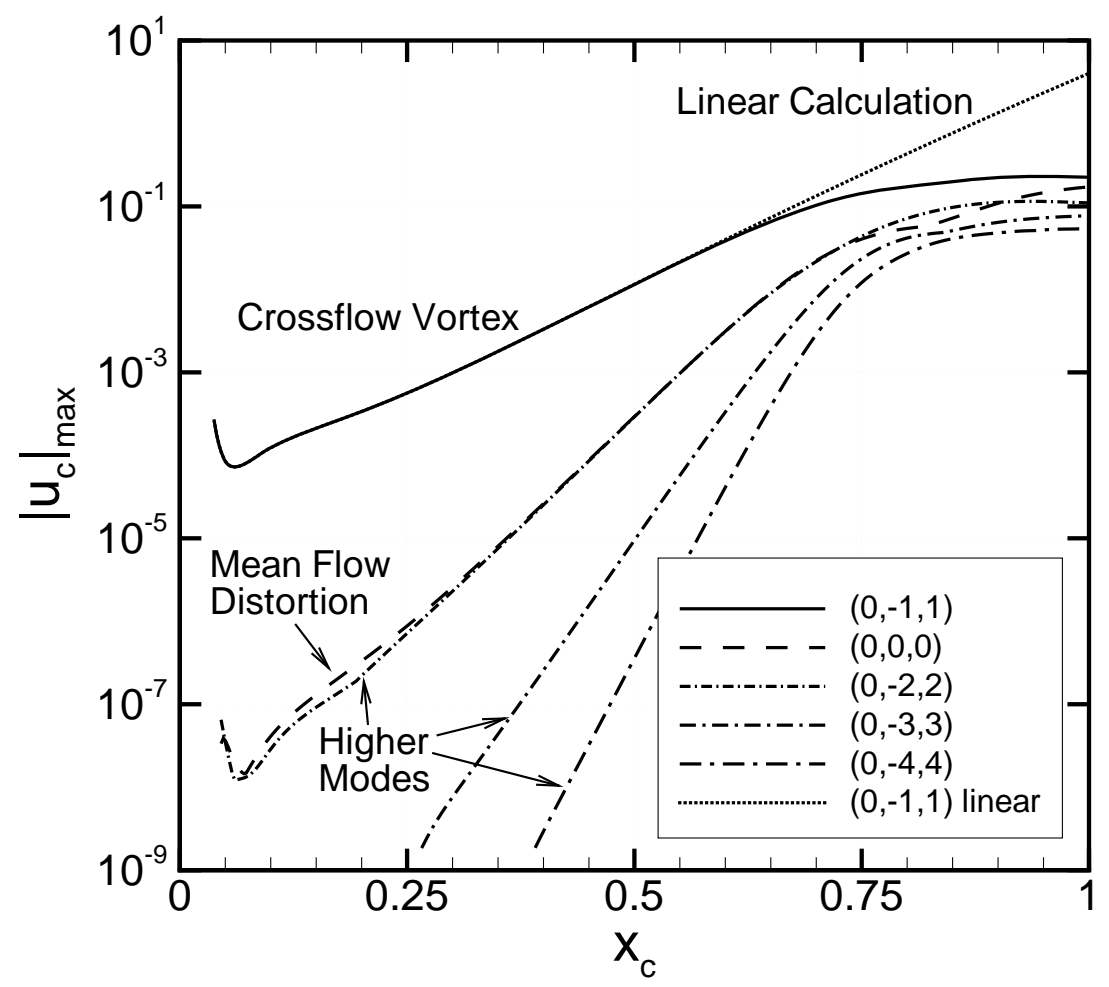

Figure 4.6: Nonlinear amplification of crossflow vortex with spanwise wave number $\beta_{c}=261.8$ and initial amplitude $\left|\tilde{u}_{c}\right|_{\max }=7.2 \cdot 10^{-5}$ at $x_{c}=0.06$. While explicit initial conditions are specified for mode $(0,-1,1)$ only, modes up to $(0,-4,4)$ are considered in the computation.

plate.

Increasing the initial amplitude of mode $(0,-1,1)$ even further to $7.2 \cdot 10^{-3}$ leads to a deviation of the vortex amplitude from the linear prediction not far downstream of the neutral point (see figure 4.8). The vortex $(0,-1,1)$ reaches an amplitude of $3 \%$ at $x_{c}=0.2$ and its maximal amplitude of $18.8 \%$ at $x_{c}=0.78$, where the amplitude of the mean flow distortion $(0,0,0)$ is $8.5 \%$. At the end of the plate the amplitude of mode $(0,-1,1)$ is decreased to $13.1 \%$, that of the mean flow distortion to $6.7 \%$. This somewhat peculiar observation - if the vortex is initialized with rather low amplitude it reaches significantly higher maximal amplitudes than if it is initialized with high amplitude - is discussed in detail in section 4.5.

For the case shown in figure 4.6 disturbance profiles of the fundamental vortex and its three harmonics are depicted in figure 4.9 at different chordwise positions. $u_{s}$ denotes the velocity component parallel to the potential streamline, $w_{s}$ the wallparallel velocity component perpendicular to the potential streamline. While the higher modes do not play an important role at $x_{c}=0.5$, at $x_{c}=0.7$ the amplitude of mode $(0,-1,1)$ is only about four times as large as that of mode $(0,-2,2)$. At $x_{c}=0.9$ the $u_{s}$-profile of mode $(0,-1,1)$ 


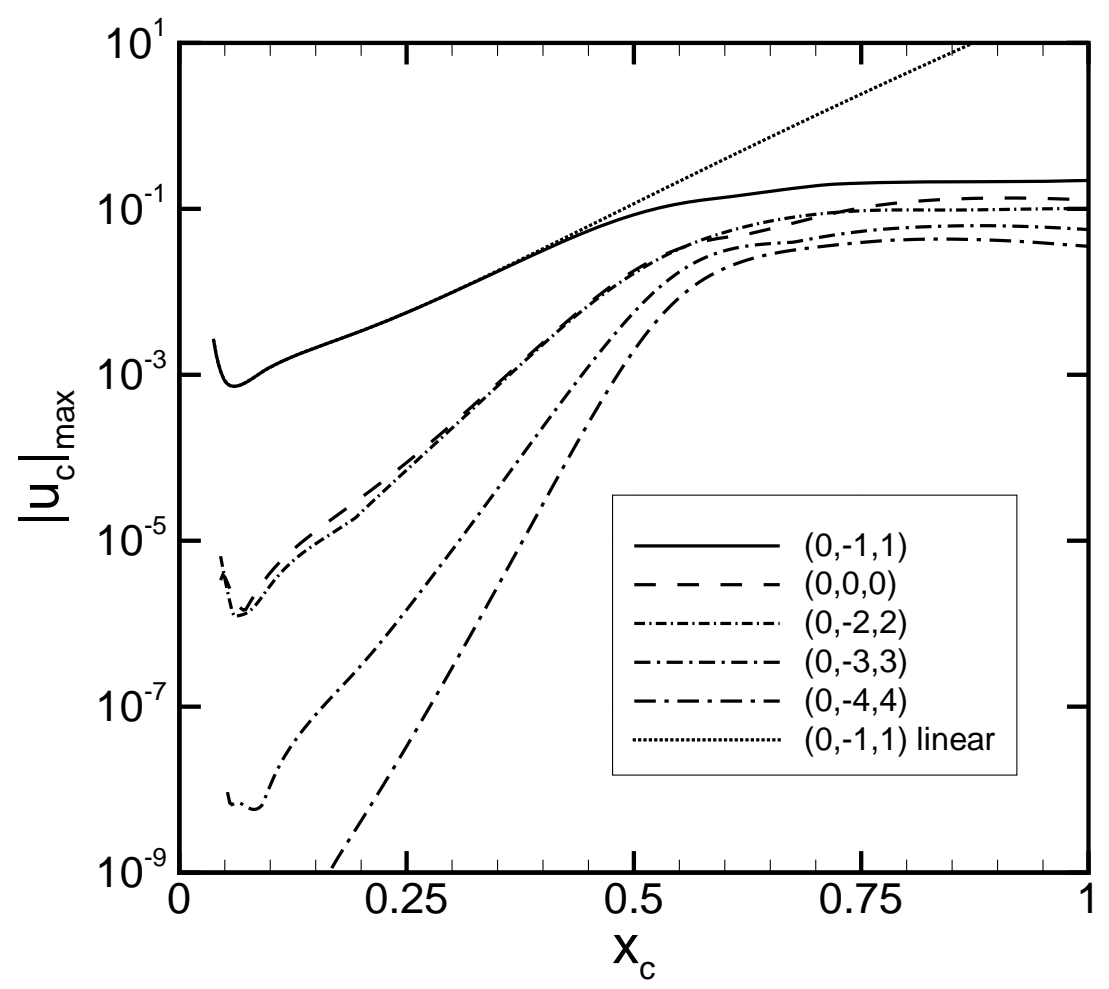

Figure 4.7: Nonlinear amplification of crossflow vortex with spanwise wave number $\beta_{c}=261.8$ and initial amplitude $\left|\tilde{u}_{c}\right|_{\max }=7.2 \cdot 10^{-4}$ at $x_{c}=0.06$. While explicit initial conditions are specified for mode $(0,-1,1)$ only, modes up to $(0,-4,4)$ are considered in the computation.

is no longer similar to profiles computed using linear instability theory. It has developed a second maximum near the edge of the boundary layer. The evolution of the nonlinear mean flow distortion and the total mean flow field is shown in figure 4.10. The $u_{s}$-component of the distorted mean flow profiles at $x_{c}=0.7$ and $x_{c}=0.9$ exhibits inflection points.

For the generation of the primarily distorted base flow for the secondary instability analysis presented in chapter 5 , the number of harmonics of the fundamental vortex is increased to 16 in the PSE-computation in order to resolve the details of the distorted base flow. In comparison with the computation for identical initial conditions and four harmonics (see figure 4.6), the amplitude of mode $(0,-1,1)$ at the end of the plate changes from $22.5 \%$ to $22.8 \%$, that of the mean flow distortion reduces from $17.2 \%$ to $16.3 \%$. As can be seen from figure 4.11, the harmonics with large spanwise wave number are generated rather far downstream in the highly nonlinear region. These modes then grow very rapidly before they saturate or even decay again. Mode $(0,-16,16)$, the highest harmonic considered in the computation, is generated at $x_{c}=0.69$, where mode $(0,-1,1)$ has reached an amplitude of $10 \%$ already and the mean flow distortion $(0,0,0)$ has an amplitude of $2 \%$. Mode $(0,-16,16)$ reaches its maximal amplitude of $6.8 \cdot 10^{-4}$ at $x_{c}=0.89$; this 


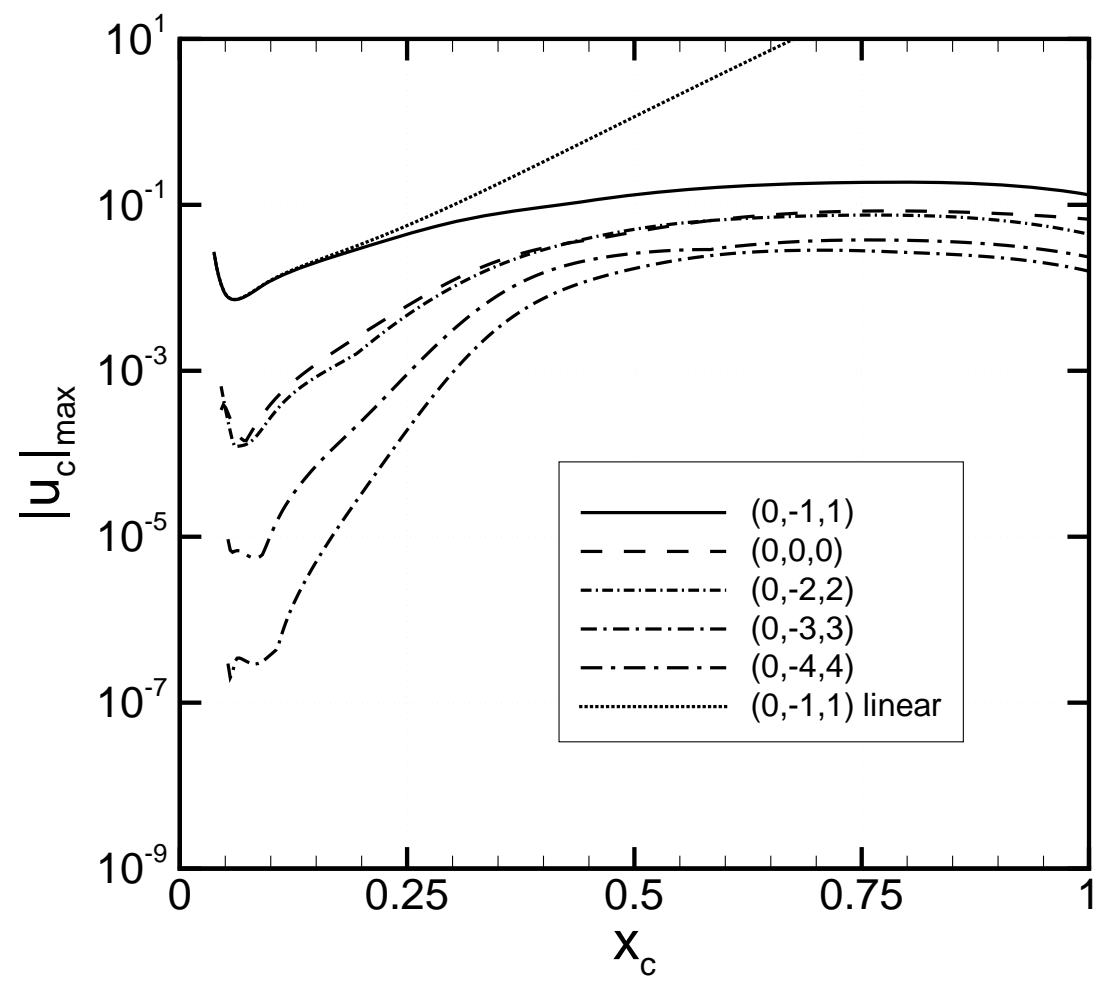

Figure 4.8: Nonlinear amplification of crossflow vortex with spanwise wave number $\beta_{c}=261.8$ and initial amplitude $\left|\tilde{u}_{c}\right|_{\max }=7.2 \cdot 10^{-3}$ at $x_{c}=0.06$. While explicit initial conditions are specified for mode $(0,-1,1)$ only, modes up to $(0,-4,4)$ are considered in the computation.

is about $0.3 \%$ of the maximal amplitude of mode $(0,-1,1)$. Further downstream the amplitude of mode $(0,-16,16)$ decreases to $3.8 \cdot 10^{-4}$ at the end of the plate.

In figures $4.12-4.14$, iso-contours of velocity components in vortex oriented coordinates are shown for different chordwise positions. The wallparallel component $u_{\psi}$ normal to the vortex axis is depicted in figure 4.12 for both the disturbance flow field (modes $(0,-1,1)-(0,-16,16)$ ) and the total flow field including the undisturbed base flow and the mean flow distortion. At $x_{c}=0.6$, the disturbance $\tilde{u}_{\psi}$ is small in comparison with the corresponding mean flow component, and the total flow field exhibits only small spanwise variation. Further downstream the disturbance amplitude and, thus, the spanwise variation of $U_{\psi}$ increase significantly.

The wallnormal velocity component $\tilde{v}$ of the disturbance is significantly larger than that of the laminar base flow at $x_{c}=0.6$ already, and it dominates the structure of the total wallnormal velocity $V$, as can be seen from figure 4.13.

By far the strongest velocity component in vortex oriented coordinates is the $w_{\psi}$-component in direction of the vortex axis, which encloses an angle 

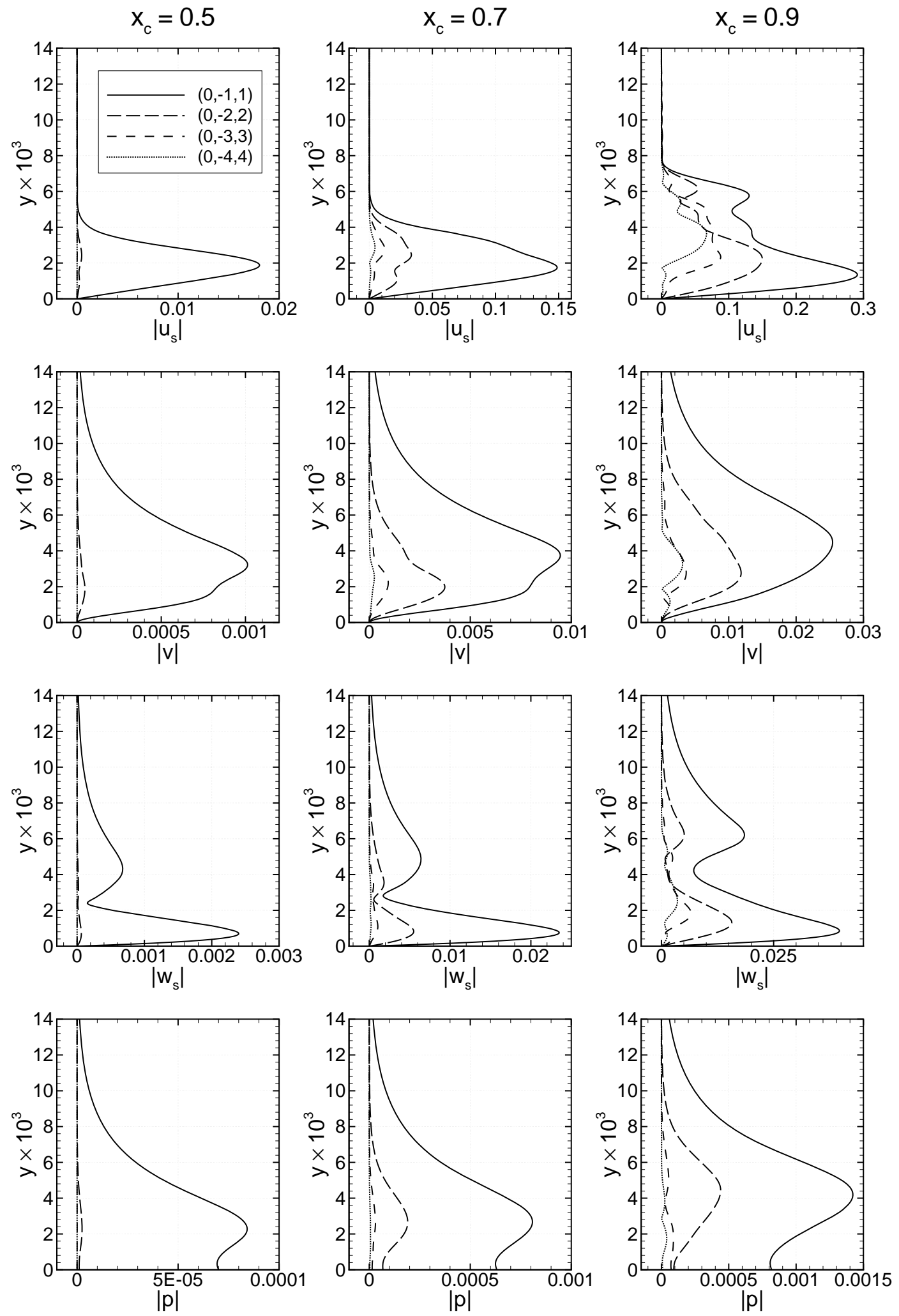

Figure 4.9: Disturbance profiles of crossflow vortex with spanwise wave number $\beta_{c}=261.8$ and harmonics at different chordwise locations. The initial amplitude of the fundamental mode is $\left|\tilde{u}_{c}\right|_{\max }=7.2 \cdot 10^{-5}$ at $x_{c}=0.06$ as in figure 4.6. 

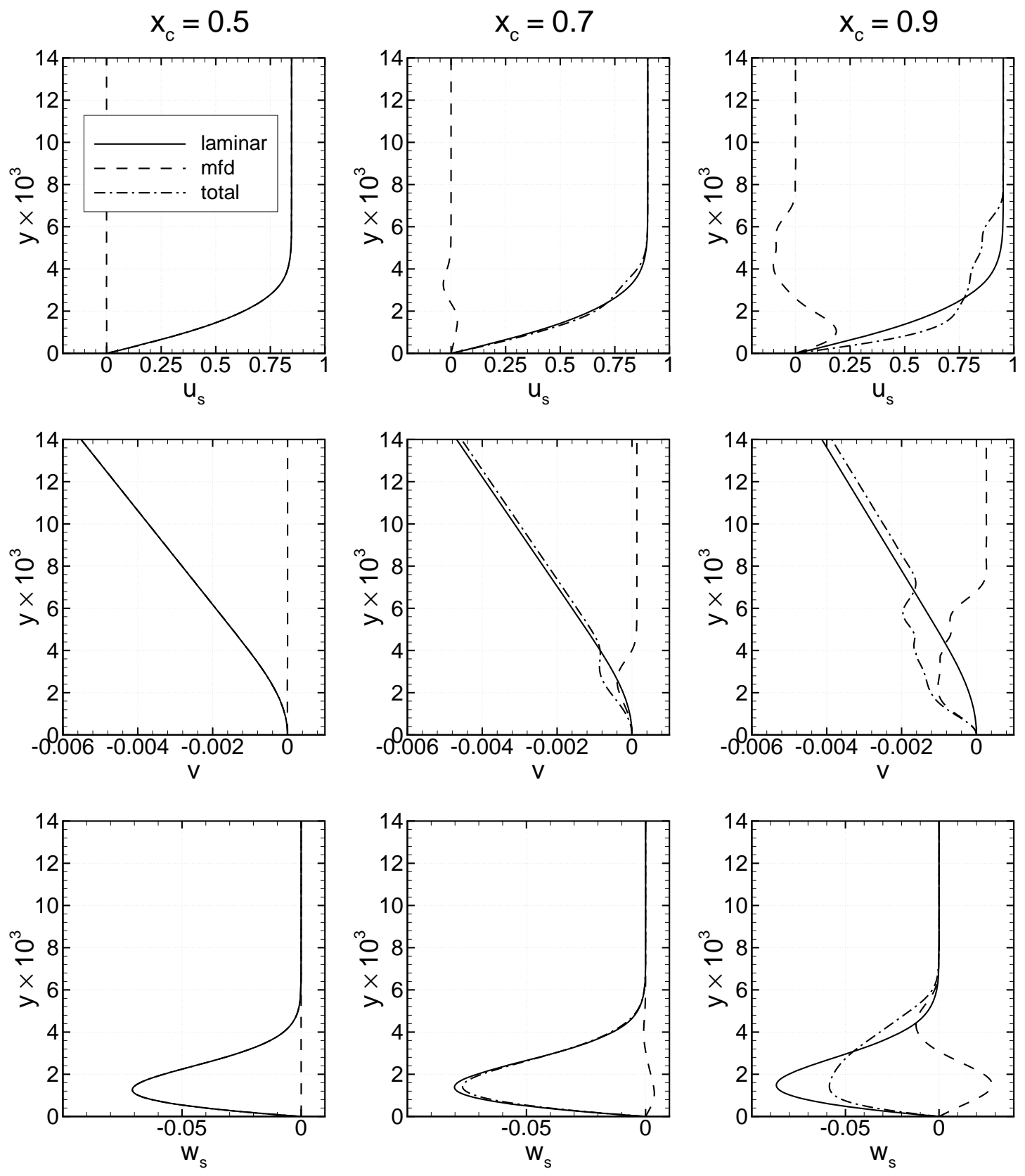

Figure 4.10: Laminar base flow, nonlinear mean flow distortion and total mean flow field as generated by a crossflow vortex with spanwise wave number $\beta_{c}=261.8$ and its first three harmonics. The initial amplitude of the vortex is $\left|\tilde{u}_{c}\right|_{\max }=7.2 \cdot 10^{-5}$ at $x_{c}=0.06$ as in figure 4.6. 


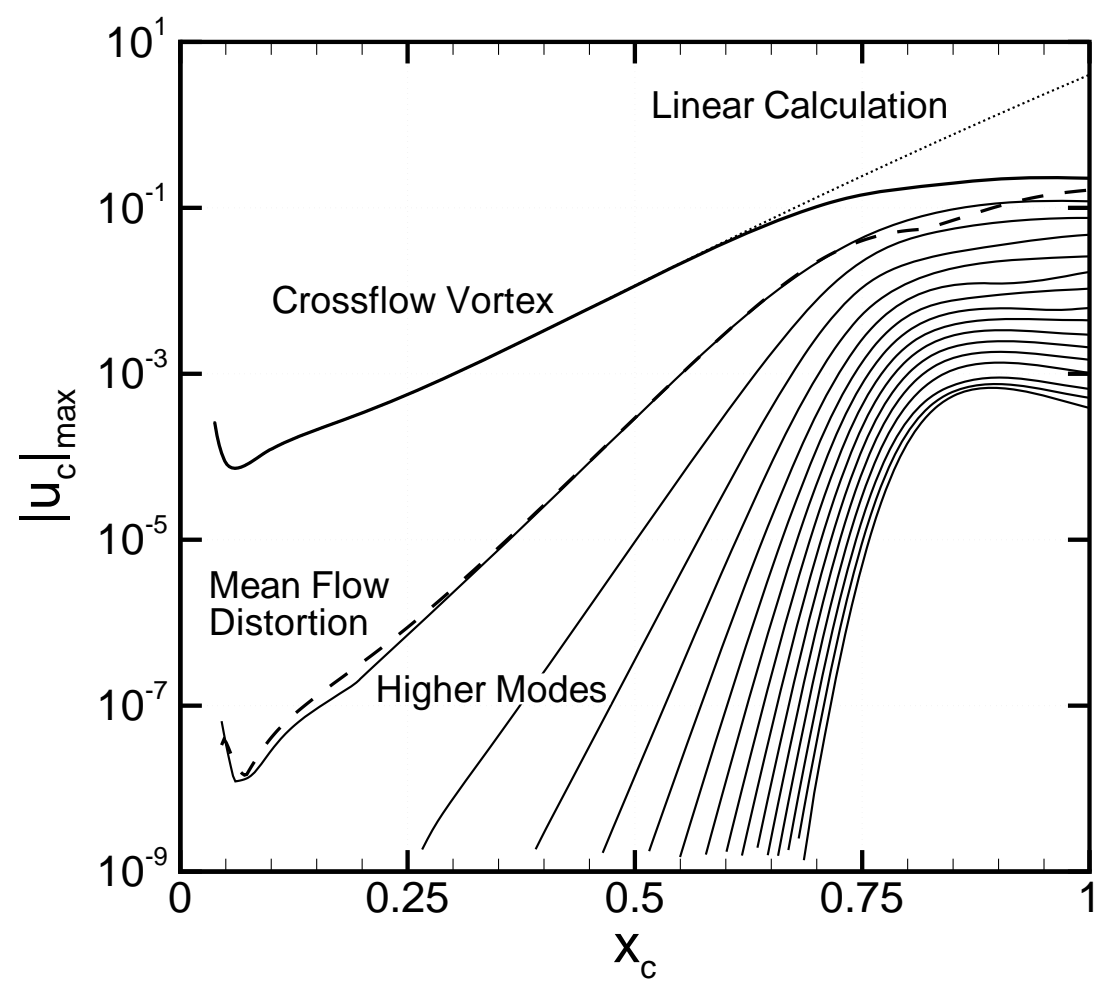

Figure 4.11: Nonlinear amplification of crossflow vortex with spanwise wave number $\beta_{c}=261.8$ and initial amplitude $\left|\tilde{u}_{c}\right|_{\max }=7.2 \cdot 10^{-5}$ at $x_{c}=0.06$. While explicit initial conditions are specified for mode $(0,-1,1)$ only, modes up to $(0,-4,4)$ are considered in the computation.

of $4.25^{\circ}$ with the potential streamline at $x_{c}=0.6$, of $3.13^{\circ}$ at $x_{c}=0.8$, and of $2.19^{\circ}$ at $x_{c}=1.0$. At $x_{c}=0.6$, the presence of the vortices leads to small spanwise variations of the velocity component $W_{\psi}$ of the total flow field (see figure 4.14). As the disturbance amplitude increases further downstream, the typical half-mushroom structure develops in the total flow field.

Due to the rotation of the crossflow vortices, low-speed fluid is moved from the near-wall region into the outer part of the boundary layer, and faster fluid is transported closer to the wall. This leads to layers of high shear $\partial W_{\psi} / \partial x_{\psi}$ and $\partial W_{\psi} / \partial y$ in the outer part of the boundary layer, which are the cause of high-frequency secondary instability near the boundary layer edge (see chapter 5).

For $x_{c}=0.8$, the velocity components perpendicular to the vortex axis are summarized in a $U_{\psi}-V$-vector plot shown in figure 4.15. Selected streamlines projected onto the $x_{\psi}-y$-plane illustrate the action of the crossflow vortices rotating in clockwise direction. In addition, they reveal the existence of small counter-clockwise rotating vortices very near to the wall. Such vortices have also been found by Malik et al. [61] in a PSE analysis of swept Hiemenz flow and by Wintergerste \& Kleiser [97] in a temporal DNS of the DLR swept plate experiment. 

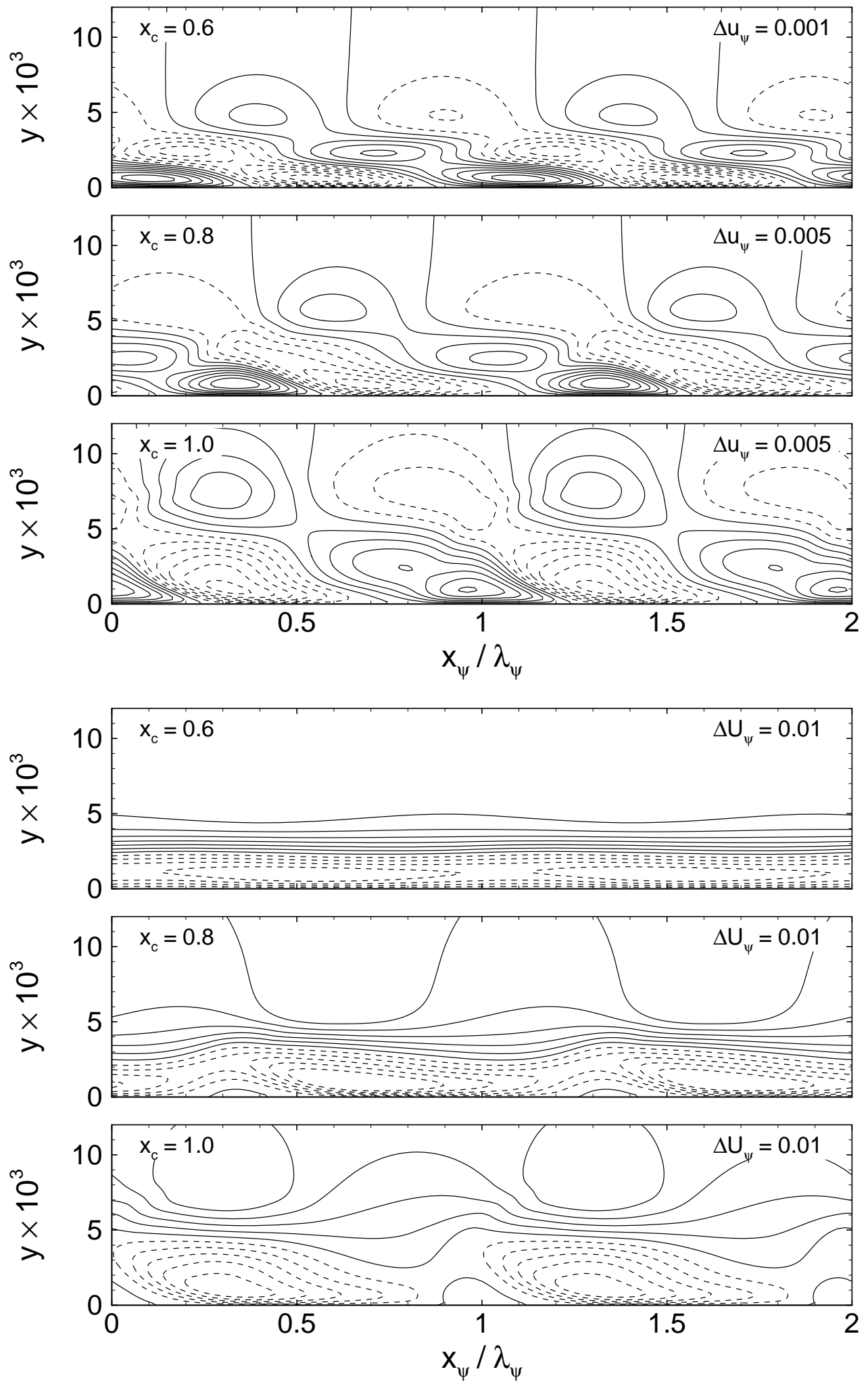

Figure 4.12: Disturbance velocity $\tilde{u}_{\psi}$ (top) and total velocity $U_{\psi}$ (bottom) in $x_{\psi}-y$-plane from PSE computation for a crossflow vortex with spanwise wave number $\beta_{c}=261.8$ and 15 harmonics (see figure 4.11). The initial amplitude of the vortex $(0,-1,1)$ is $\left|\tilde{u}_{c}\right|_{\text {max }}=7.2 \cdot 10^{-5}$ at $x_{c}=0.06$. Dashed lines indicate negative values. 

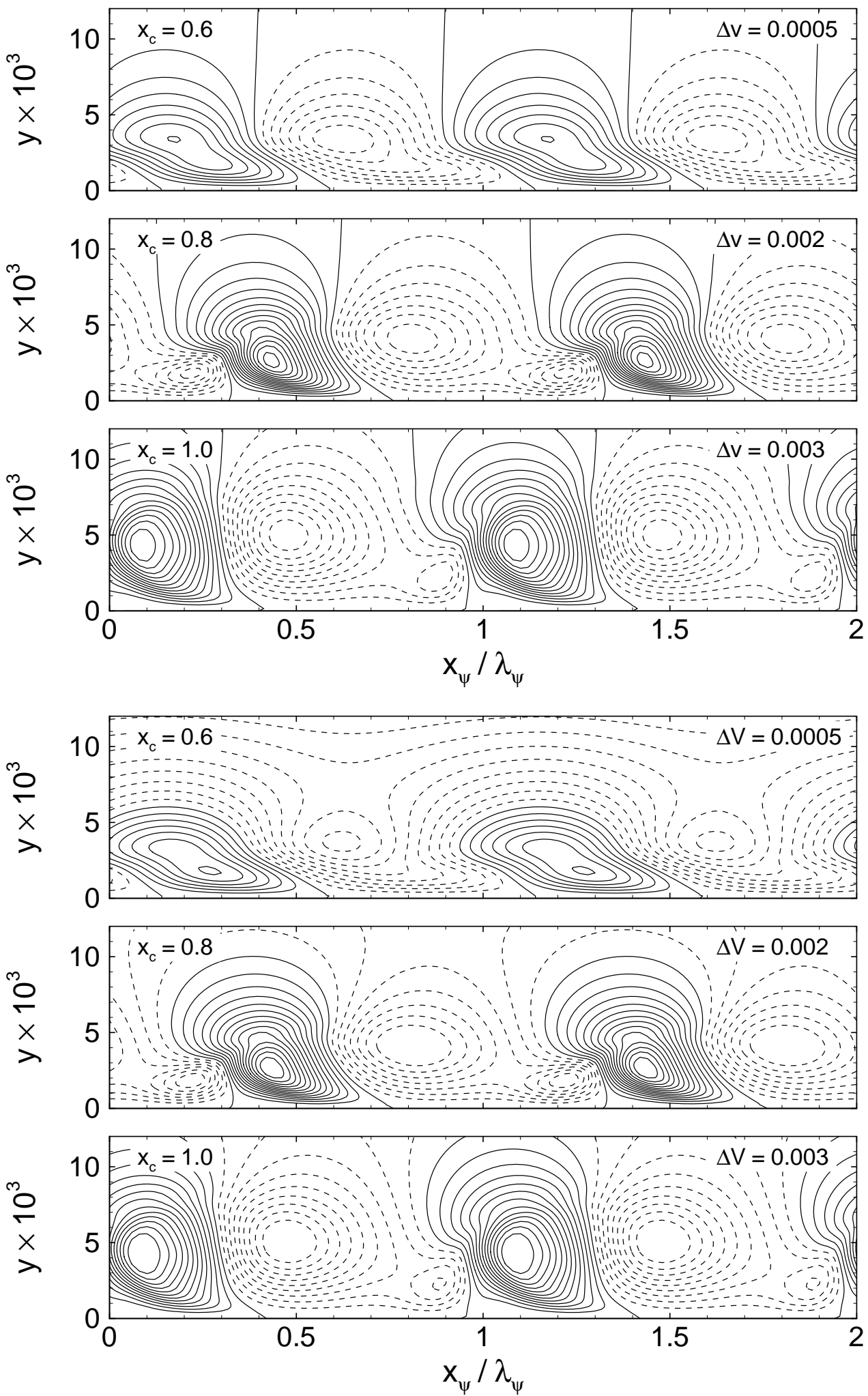

Figure 4.13: Disturbance velocity $\tilde{v}$ (top) and total velocity $V$ (bottom) in $x_{\psi}-y$-plane from PSE computation for a crossflow vortex with spanwise wave number $\beta_{c}=261.8$ and 15 harmonics (see figure 4.11). The initial amplitude of the vortex $(0,-1,1)$ is $\left|\tilde{u}_{c}\right|_{\text {max }}=7.2 \cdot 10^{-5}$ at $x_{c}=0.06$. Dashed lines indicate negative values. 

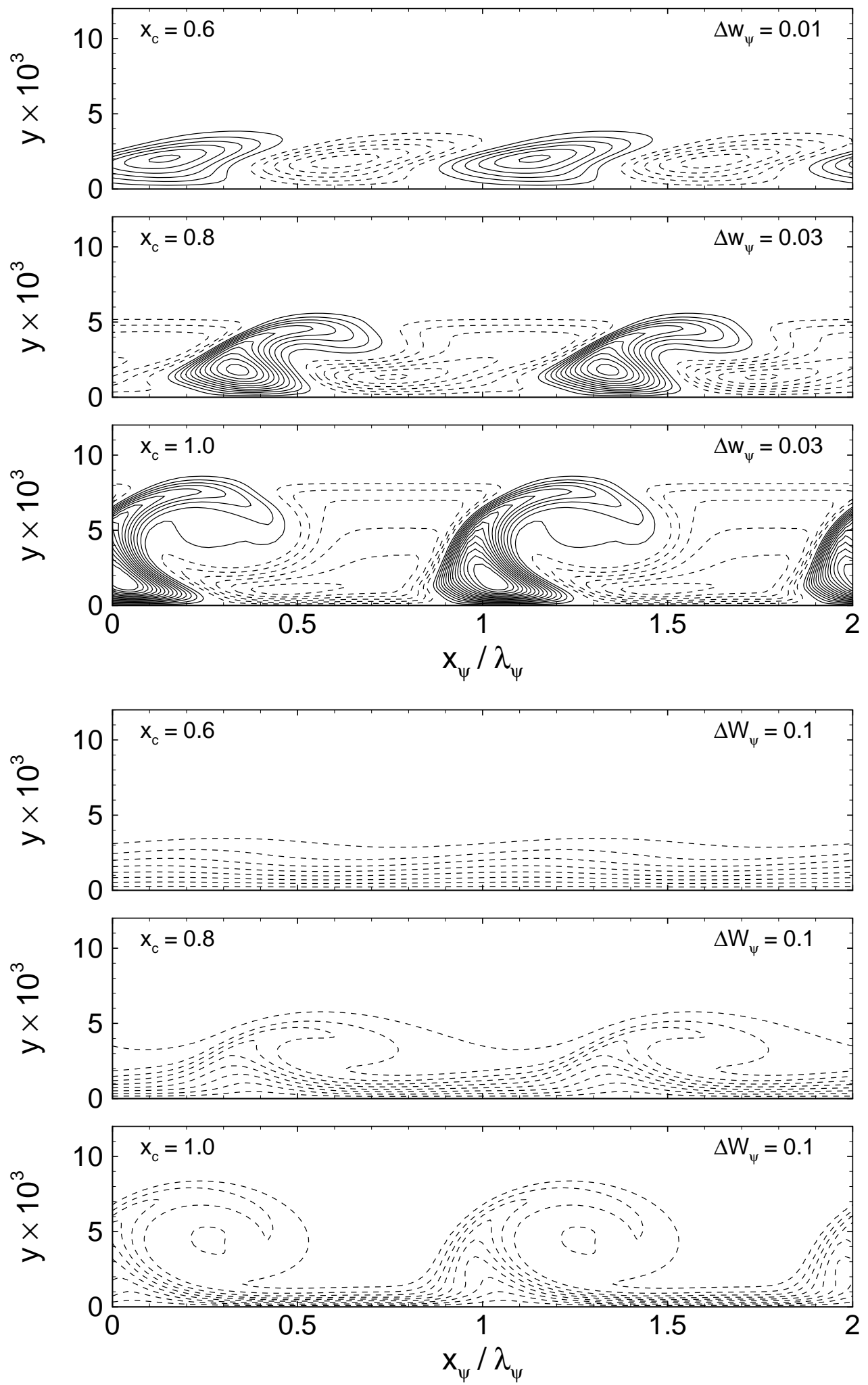

Figure 4.14: Disturbance velocity $\tilde{w}_{\psi}$ (top) and total velocity $W_{\psi}$ (bottom) in $x_{\psi}-y$-plane from PSE computation for a crossflow vortex with spanwise wave number $\beta_{c}=261.8$ and 15 harmonics (see figure 4.11). The initial amplitude of the vortex $(0,-1,1)$ is $\left|\tilde{u}_{c}\right|_{\text {max }}=7.2 \cdot 10^{-5}$ at $x_{c}=0.06$. Dashed lines indicate negative values. 


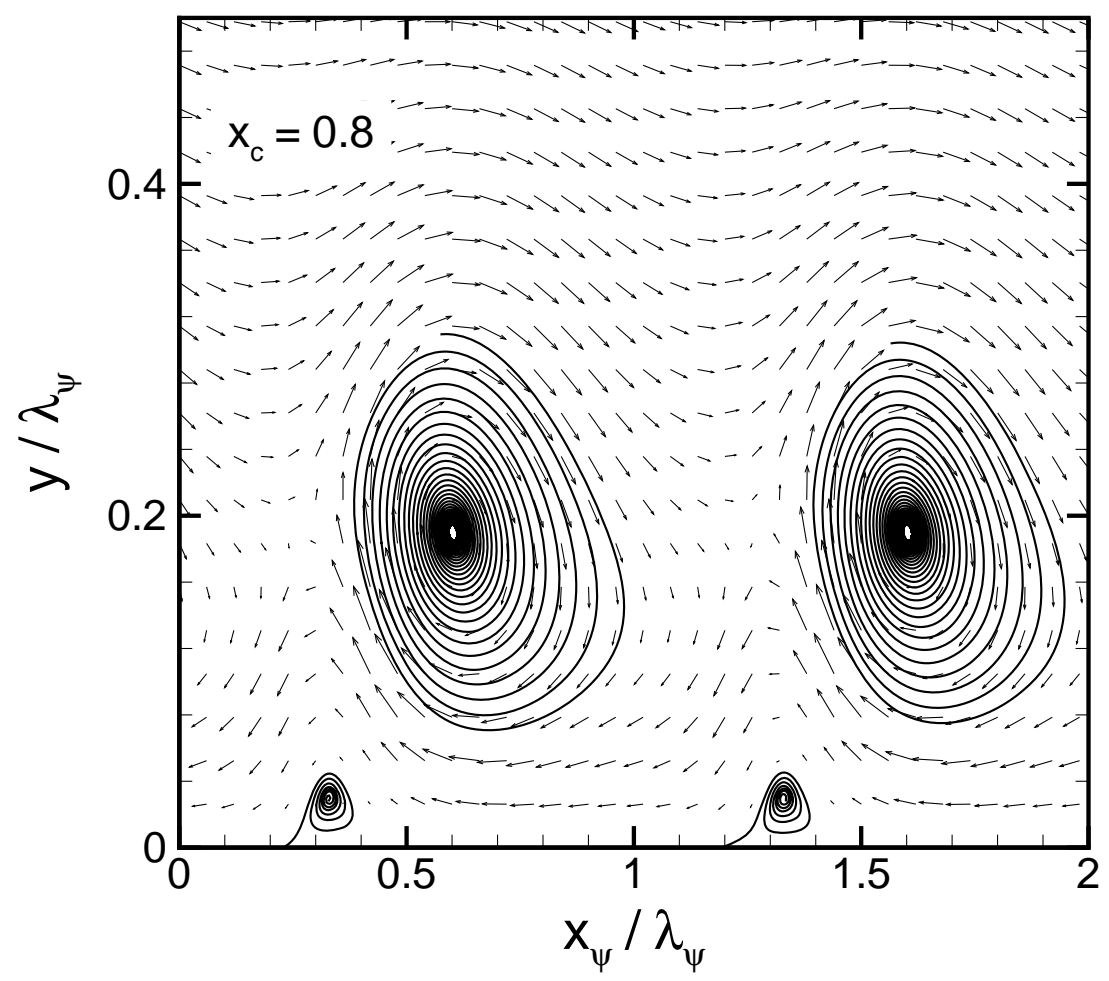

Figure 4.15: $U_{\psi}-V$-vector plot and streamlines projected onto $x_{\psi}-y$-plane at $x_{c}=0.8$ from PSE computation for a crossflow vortex with spanwise wave number $\beta_{c}=261.8$ and 15 harmonics. The initial amplitude of the vortex $(0,-1,1)$ is $\left|\tilde{u}_{c}\right|_{\max }=7.2 \cdot 10^{-5}$ at $x_{c}=0.06$. Modes up to $(0,-16,16)$ are considered in the computation.

\subsection{Wave-Vortex Interaction}

While stationary vortices originate from surface roughness, traveling crossflow waves are generated by free stream turbulence. B. Müller [65] experimentally observed that the presence of traveling waves can lower the amplification of steady vortices. This observation was confirmed in temporal numerical simulations by Wagner [94], in spatial numerical simulations by W. Müller [67], in large-eddy simulations by Huai et al. [43], and in investigations using the PSE by Malik et al. [61] and by Bertolotti [8]. Deyhle \& Bippes [18] showed that high turbulence levels $(T u>0.2 \%)$ and smooth surfaces are necessary for the traveling waves to dominate the transition process.

The interaction of traveling and stationary crossflow disturbances is discussed in this section for a crossflow vortex with spanwise wave number $\beta_{c}=261.8$ and its first three harmonics, as considered in section 4.3. The traveling wave has the same spanwise wave number as the vortex, the dimensional frequency of this mode is chosen to be $f^{*}=135 \mathrm{~Hz}$, as in the 
spatial numerical simulation of W. Müller [67]. This corresponds to a nondimensional frequency of $f=3.553$. As can be seen from figure 3.10, this mode exhibits significantly higher growth rates than the stationary vortex. The chordwise wave number $\alpha_{c}$ of the traveling wave is about 0.75 times that of the vortex at all chordwise positions. Hence, the vortex is denoted by $(0,-4,1)$, the wave by $(1,-3,1)$. Due to nonlinear forcing, a mean flow distortion $(0,0,0)$ and the harmonics of both modes, i.e. the modes $(0,-8,2)$, $(0,-12,3),(0,-16,4)$ and $(2,-6,2),(3,-9,3),(4,-12,4)$, are generated. In addition, the interaction of these modes yields many other disturbance modes like $(1,-7,2)$ and $(1,1,0)$. With spectrum limits $(L, N, K)=(4,20,4)$, as used in the computations presented in this section, the set of nonlinearly generated disturbance modes is summarized in table 4.1.

\begin{tabular}{|c||}
\hline \hline$(0,-4,1)(1,-3,1)$ \\
\hline$(0,0,0)(0,-8,2)(1,1,0)(1,-7,2)(2,-6,2)$ \\
\hline$(0,-12,3)(0,-16,4)(1,9,-2)(1,5,-1)(1,-11,3)(1,-15,4)(2,2,0)$ \\
$(2,-2,1)(2,-10,3)(2,-14,4)(3,-5,2)(3,-9,3)(3,-13,4)(4,-12,4)$ \\
\hline$(1,17,-4)(1,13,-3)(2,18,-4)(2,14,-3)(2,10,-2)(2,6,-1)(3,11,-2)$ \\
$(3,7,-1)(3,3,0)(3,-1,1)(4,4,0)(4,0,1)(4,-4,2)(4,-8,3)$ \\
\hline$(3,19,-4)(3,15,-3)(4,20,-4)(4,16,-3)(4,12,-2)(4,8,-1)$ \\
\hline \hline
\end{tabular}

Table 4.1: Modes generated in different stages of nonlinear interaction between stationary crossflow vortex $(0,-4,1)$ and traveling wave $(1,-3,1)$ for spectrum limits $(L, N, K)=(4,20,4)$.

The index vectors $\mathbf{p}=(l, n, k)$ of all modes present in the computations are linear combinations of the index vectors $\mathbf{p}_{\text {wave }}=(1,-3,1)$ and $\mathbf{p}_{\text {vortex }}=$ $(0,-4,1)$. Hence, they lie on a plane spanned by $\mathbf{p}_{\text {wave }}$ and $\mathbf{p}_{\text {vortex }}$ in index space. The projection of this plane onto the $l-k$-plane, as shown in figure 4.16, still allows a unique identification of all modes involved in the interaction. While the index $n$ for the chordwise wave number $\alpha_{c}$ is not necessary to identify a disturbance mode in this type of wave-vortex interaction, as a consequence of equation 4.6, it is a good guide to estimate the local phase speed

$$
c_{(l, n, k)}\left(x_{c}\right) \approx \frac{l}{n} \frac{\omega_{0}}{\alpha_{c, p h y s, 0}\left(x_{c}\right)}
$$

and propagation direction

$$
\psi_{(l, n, k)}\left(x_{c}\right) \approx \arctan \left(\frac{k}{n} \frac{\beta_{c, 0}}{\alpha_{c, p h y s, 0}\left(x_{c}\right)}\right) .
$$

Negative $n$ corresponds to a mode traveling towards the leading edge. 


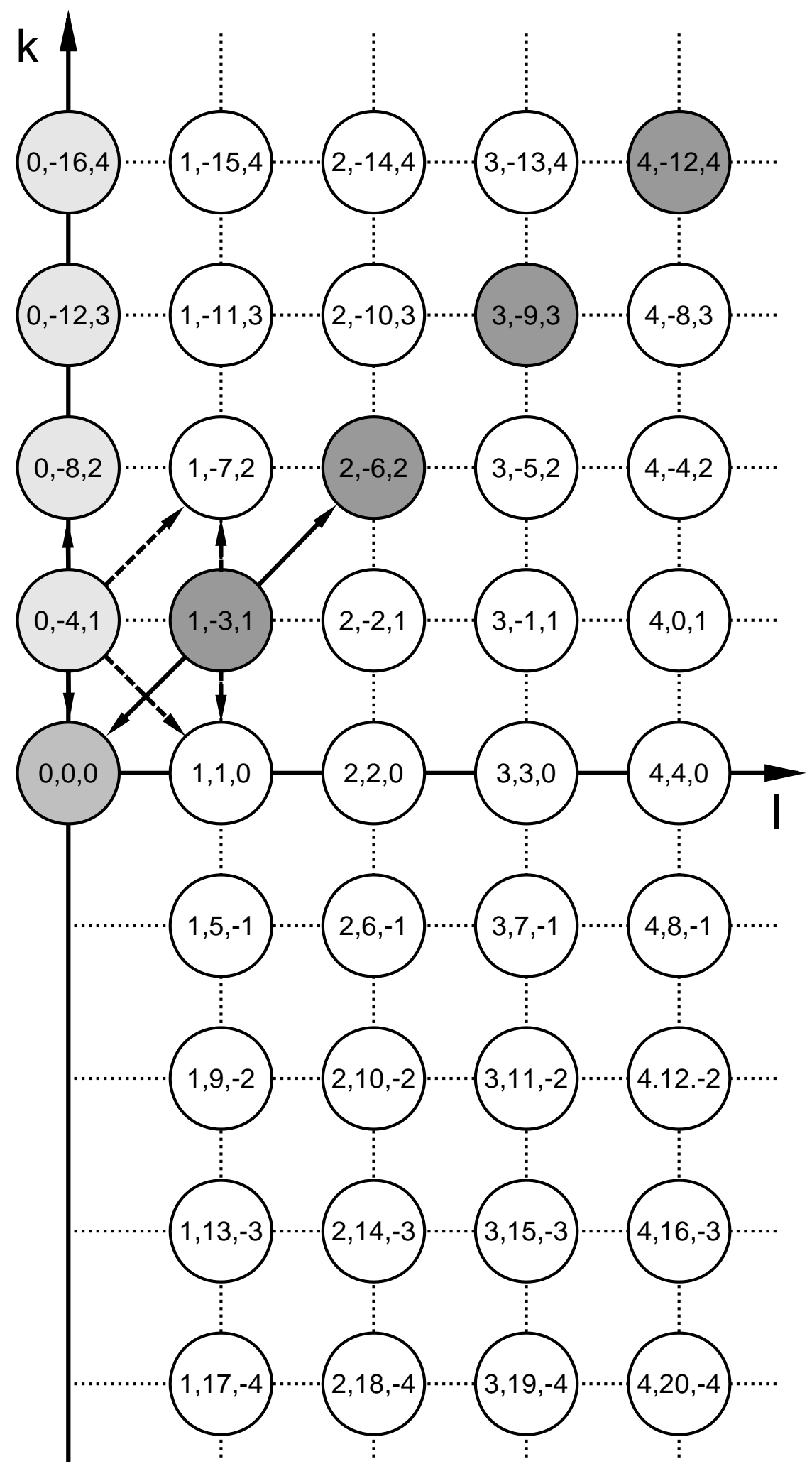

Figure 4.16: Nonlinear interaction between wave $(1,-3,1)$ and vortex $(0,-4,1)$ in index space. The nonlinear forcing in the first interaction stage is indicated by arrows. 
In the interaction scenarios shown in figures $4.17-4.19$, the crossflow vortex is initialized with an amplitude $\left|\tilde{u}_{c}\right|_{\max }=7.2 \cdot 10^{-5}$ as in the computation presented in figure 4.6. In environments with very low free stream turbulence, the traveling waves can be expected to have significantly lower initial amplitudes than the stationary vortices. Such a case is shown in figure 4.17, where the wave $(1,-3,1)$ is initialized such that at its neutral point at $x_{c}=0.065$ it has an amplitude $\left|\tilde{u}_{c}\right|_{\text {max }}=4.2 \cdot 10^{-7}$.

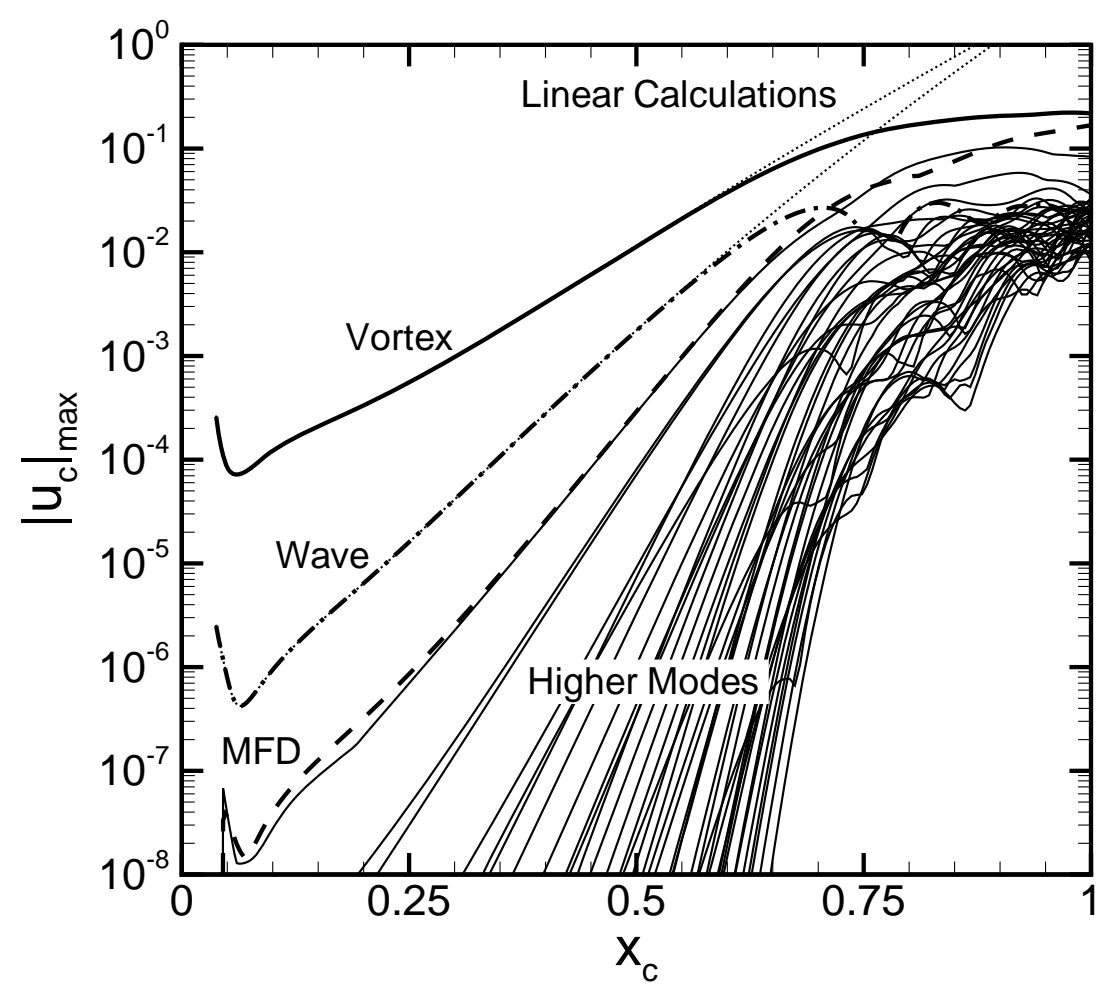

Figure 4.17: Interaction between a crossflow vortex with spanwise wave number $\beta_{c}=261.8$ and a traveling crossflow mode with the same spanwise wave number and frequency $f=3.553$. The initial amplitude $\left|\tilde{u}_{c}\right|_{\max }$ of the vortex is $7.2 \cdot 10^{-5}$ at $x_{c}=0.06$ as in figure 4.6 , that of the wave is $4.2 \cdot 10^{-7}$ at its neutral point at $x_{c}=0.065$.

At this low amplitude, a significant influence of the wave on the evolution of the vortex cannot be expected. Both disturbances grow as predicted by linear PSE computations. Due to its far higher amplitude, the vortex dominates the forcing of the mean flow distortion. The vortex $(0,-8,2)$ is forced at similar amplitude as the mean flow distortion, while modes $(1,-7,2)$ and $(1,1,0)$ experience much less forcing, and the wave $(2,-6,2)$ does not reach an amplitude of $10^{-8}$ before $x_{c}=0.33$, where the fundamental wave has an amplitude of $8 \cdot 10^{-5}$. The wave $(1,-3,1)$ grows faster than the vortex $(0,-4,1)$ up to $x_{c} \approx 0.6$, where nonlinear saturation sets in. As in the case without waves, the fundamental vortex reaches an amplitude of $3 \%$ at $x_{c}=0.58$ and starts to deviate from the linear prediction. Even though the wave $(1,-3,1)$ has 


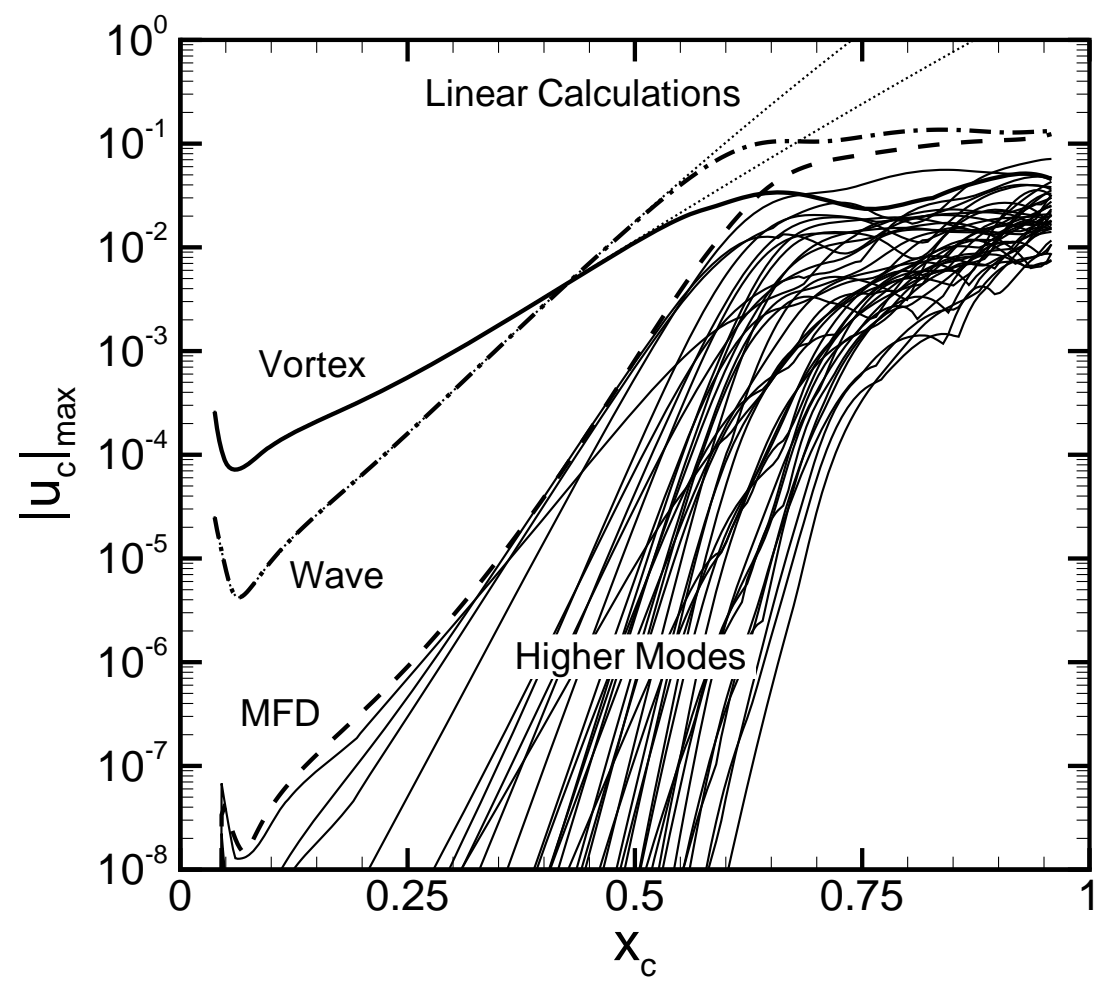

Figure 4.18: Interaction between a crossflow vortex with spanwise wave number $\beta_{c}=261.8$ and a traveling crossflow mode with the same spanwise wave number and frequency $f=3.553$. The initial amplitude $\left|\tilde{u}_{c}\right|_{\text {max }}$ of the vortex is $7.2 \cdot 10^{-5}$, that of the wave is $4.2 \cdot 10^{-6}$.

reached an amplitude of only $0.7 \%$ at this location, its growth rate also reduces in comparison with linear theory. At $x_{c}=0.7$, this mode reaches an amplitude maximum of $2.7 \%$. While the vortex $(0,-4,1)$ continues to grow, the amplitude of wave $(1,-3,1)$ starts to oscillate indicating a quasi-equilibrium state. The maximal amplitude of the fundamental vortex is $22.2 \%$ at $x_{c}=0.98$, where the mean flow distortion $(0,0,0)$ has an amplitude of $15.6 \%$.

The comparison between these results and the case where only vortices are considered shows, that the evolution of vortices and mean flow distortion is not affected very much by the presence of crossflow waves after the vortices have reached high amplitudes. A mean flow strongly distorted by high amplitude crossflow vortices suppresses the growth of crossflow waves despite of their higher growth rates according to linear instability theory. However, this does not justify the neglect of traveling waves in quiet environments. In spite of the low initial amplitude of mode $(1,-3,1)$, all modes present in the computation have amplitudes above $0.5 \%$ at the end of the plate.

A completely different picture arises, if the traveling wave is initialized at a ten times higher amplitude of $4.2 \cdot 10^{-6}$. This scenario is shown in figure 4.18. Only in the front part of the plate, the flow field is dominated by the vortex $(0,-4,1)$ which is initialized at an amplitude of $7.2 \cdot 10^{-5}$. Due to its higher 


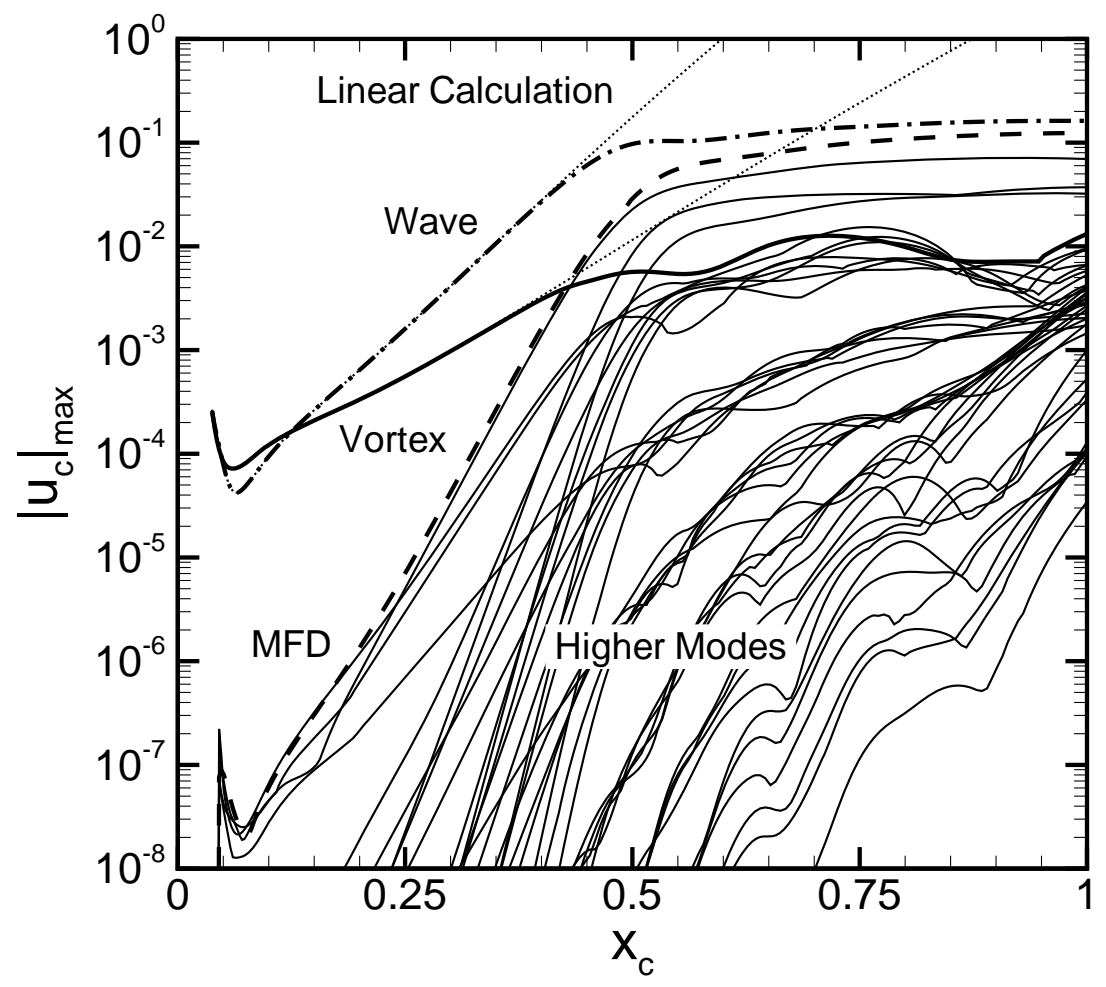

Figure 4.19: Interaction between a crossflow vortex with spanwise wave number $\beta_{c}=261.8$ and a traveling crossflow mode with the same spanwise wave number and frequency $f=3.553$. The initial amplitude $\left|\tilde{u}_{c}\right|_{\max }$ of the vortex is $7.2 \cdot 10^{-5}$, that of the wave is $4.2 \cdot 10^{-5}$.

growth rate in the linear regime, the wave $(1,-3,1)$ surpasses the vortex in amplitude at $x_{c}=0.43$, where both modes have amplitudes of about $0.5 \%$. At $x_{c}=0.53$, the wave reaches an amplitude of $3 \%$, and the growth rates of wave and vortex start to decrease. A first amplitude maximum of $10.6 \%$ is reached by the wave at $x_{c}=0.66$, where the vortex reaches an amplitude maximum as well. However, the amplitude of the vortex $(0,-4,1)$ is only $3.4 \%$; it saturates at a much lower amplitude than in the case without waves present in the flow field. At $x_{c}=0.66$, the mean flow distortion has an amplitude of $4.8 \%$. While this mode continues to grow monotonically further downstream, the amplitudes of both vortex and wave start to oscillate at $x_{c}=0.66$. The amplitudes at the maxima increase to $5.1 \%$ at $x_{c}=0.91$ for the vortex and $13.6 \%$ at $x_{c}=0.84$ for the wave.

If the initial amplitude of the wave $(1,-3,1)$ is again increased by a factor of ten to $4.2 \cdot 10^{-5}$ (see figure 4.19 ), it exceeds the vortex $(0,-4,1)$ at $x_{c}=0.13$, where both modes have an amplitude of $1.7 \cdot 10^{-4}$. The wave reaches $3 \%$ amplitude at $x_{c}=0.41$, where the fundamental vortex has grown to $0.33 \%$, and the mean flow distortion has an amplitude of $0.21 \%$. In spite of the low amplitude of the mean flow distortion the vortex growth already starts to decrease in comparison with the linear prediction. The amplitude of the 
vortex reaches a first maximum of $0.57 \%$ at $x_{c}=0.51$, and it decreases slightly to $0.54 \%$ at $x_{c}=0.56$ before it increases to its second maximum of $1.3 \%$ at $x_{c}=0.72$. It then again increases to $0.71 \%$ at $x_{c}=0.88$ and raises to $1.4 \%$ at the end of the plate.

In summary, the presence of high amplitude traveling waves in the flow field leads to amplitudes of the fundamental vortex, which are about by a factor of 20 smaller than in the case without waves present in the flow field. These results are in agreement with the findings of Malik et al. [61] and of Bertolotti [8].

At $x_{c}=0.7$, the wave $(1,-3,1)$ has an amplitude of $13.5 \%$, the mean flow distortion has reached $9 \%$. The amplitude of the wave increases to $16.2 \%$, that of the mean flow distortion to $12.4 \%$ at the end of the plate. As a result of the strong suppression of the vortex $(0,-4,1)$, the harmonics of this mode and all modes generated by nonlinear interaction between waves and vortices reach comparably low amplitudes as well. Even mode $(4,-12,4)$, the highest harmonic of the fundamental wave $(1,-3,1)$ which is inside the spectrum limits of the computation, with an amplitude of $3.2 \%$ at the end of the plate clearly exceeds all modes forced by vortex-vortex or wave-vortex interaction.

Iso-contours of the velocity component $u_{s}$ in direction of the potential streamline (the orientation of the vortex axis differs slightly between the runs with different wave amplitudes) are compared in figures 4.20 and 4.21 for the different cases. Figure 4.20 shows the contribution of the vortices $(0,-4,1)-(0,-16,4)$ to the flow field at $x_{c}=0.8$. If the traveling wave is initialized with an amplitude of $4.2 \cdot 10^{-7}$, not only the amplitudes of the vortices but also the velocity profiles of these disturbances are very similar to those in the case without waves present in the flow field. In the cases dominated by traveling waves, the $\tilde{u}_{s}$-profiles develop a clear second maximum further away from the wall. This second maximum of $\left|\tilde{u}_{s}\right|$ even exceeds the near wall maximum of the disturbance velocity in the case with high initial amplitude of the traveling wave. As a result of the reduced vortex amplitude, the spanwise variation of the total stationary flow field decreases very much with increasing initial amplitude of the traveling wave as can be seen from figure 4.21, where in addition to the vortices the nonlinear mean flow distortion and the laminar base flow are considered for the computation of the flow field.

The amplitude evolution of the stationary vortices is summarized in figure 4.22 for the different interaction cases. In this plot, the total fluctuation energy per unit mass

$$
E\left(x_{c}\right)=2 \sum_{\mathbf{p} \in \mathcal{P}_{v}} \int_{0}^{y_{e}}\left\{\left|\hat{u}_{\mathbf{p}}\left(x_{c}, y\right)\right|^{2}+\left|\hat{v}_{\mathbf{p}}\left(x_{c}, y\right)\right|^{2}+\left|\hat{w}_{\mathbf{p}}\left(x_{c}, y\right)\right|^{2}\right\} d y
$$

with

$$
\mathcal{P}_{\boldsymbol{v}}=\{(0,-4,1),(0,-8,2),(0,-12,3),(0,-16,4)\}
$$



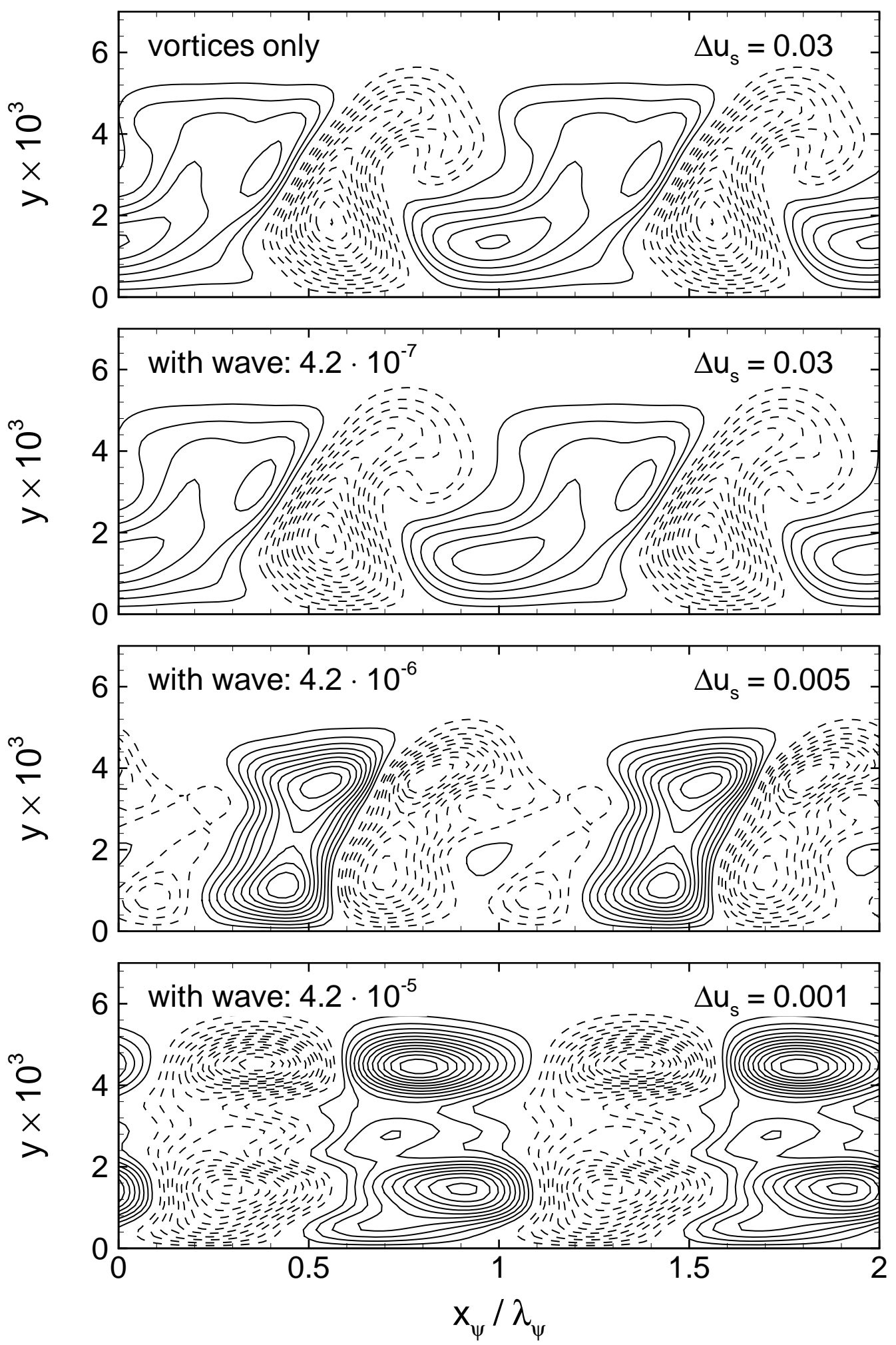

Figure 4.20: Contribution of the vortices $(0,-4,1)-(0,-16,4)$ to the flow field at $x_{c}=0.8$ for different initial amplitudes of the traveling wave. Plotted are iso-contours of the velocity component in direction of the potential streamline. Dashed lines indicate negative values. 

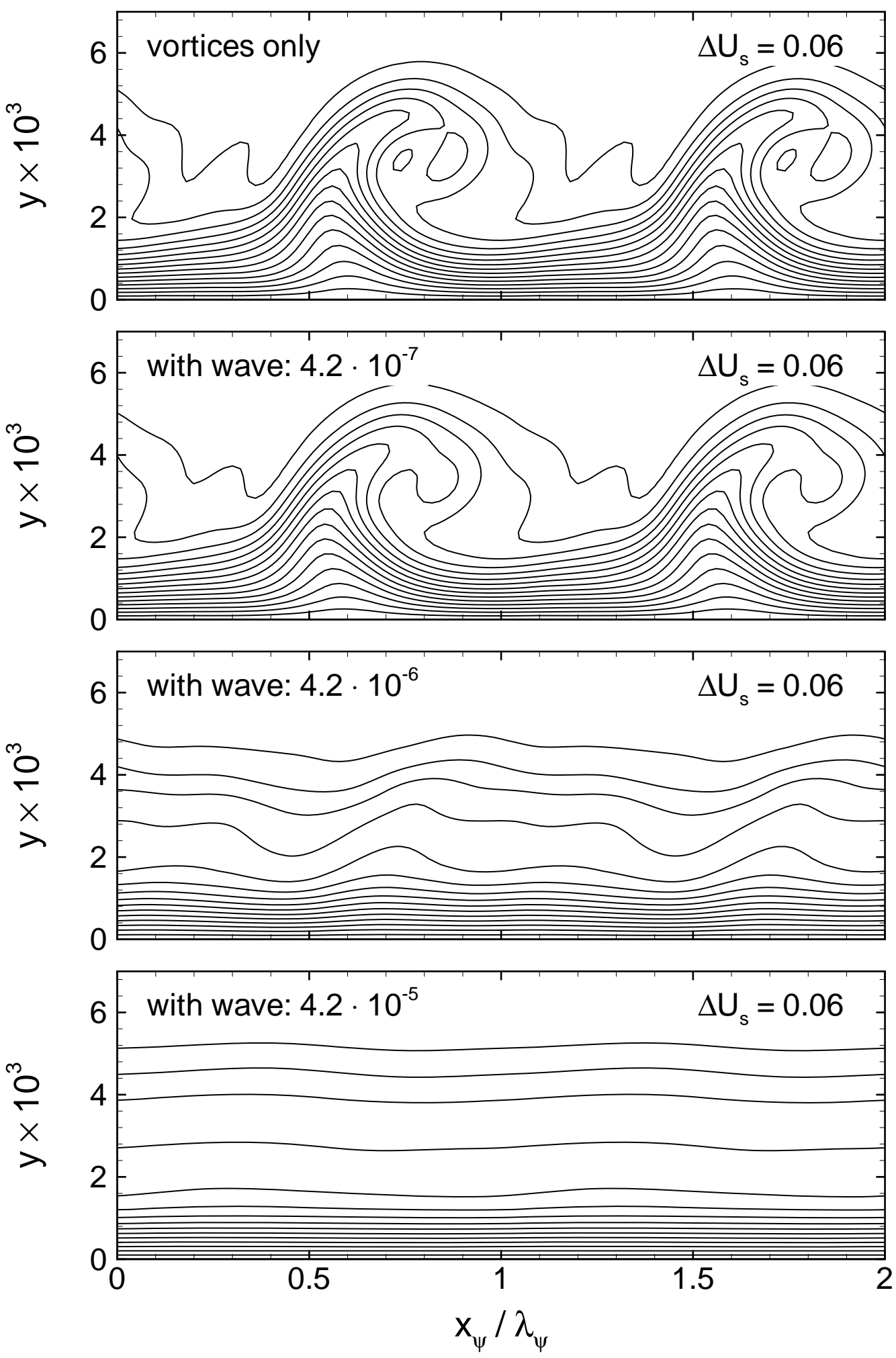

Figure 4.21: Stationary part of total velocity $U_{s}$ at $x_{c}=0.8$ from PSE computations for a wave-vortex interaction with different initial amplitudes of the traveling wave. 
is used to combine the contribution of all vortex modes into one measure of vortex amplitude. In addition to the reduction of vortex amplitude by the the presence of traveling waves, this plot illustrates the oscillatory behavior of the vortex amplitude in the nonlinear stage indicating a quasi-equilibrium state. A similar phenomenon - the reduction of disturbance amplitude in a region with high growth rates predicted by linear theory - has also been observed in section 4.3 with only stationary vortices present in the flow field. The term "quasi-equilibrium state" must, however, be used with great care, since the properties of the laminar base flow change with the chordwise coordinate $x_{c}$. In the next section these features of vortex amplitude "saturation" are discussed in more detail.

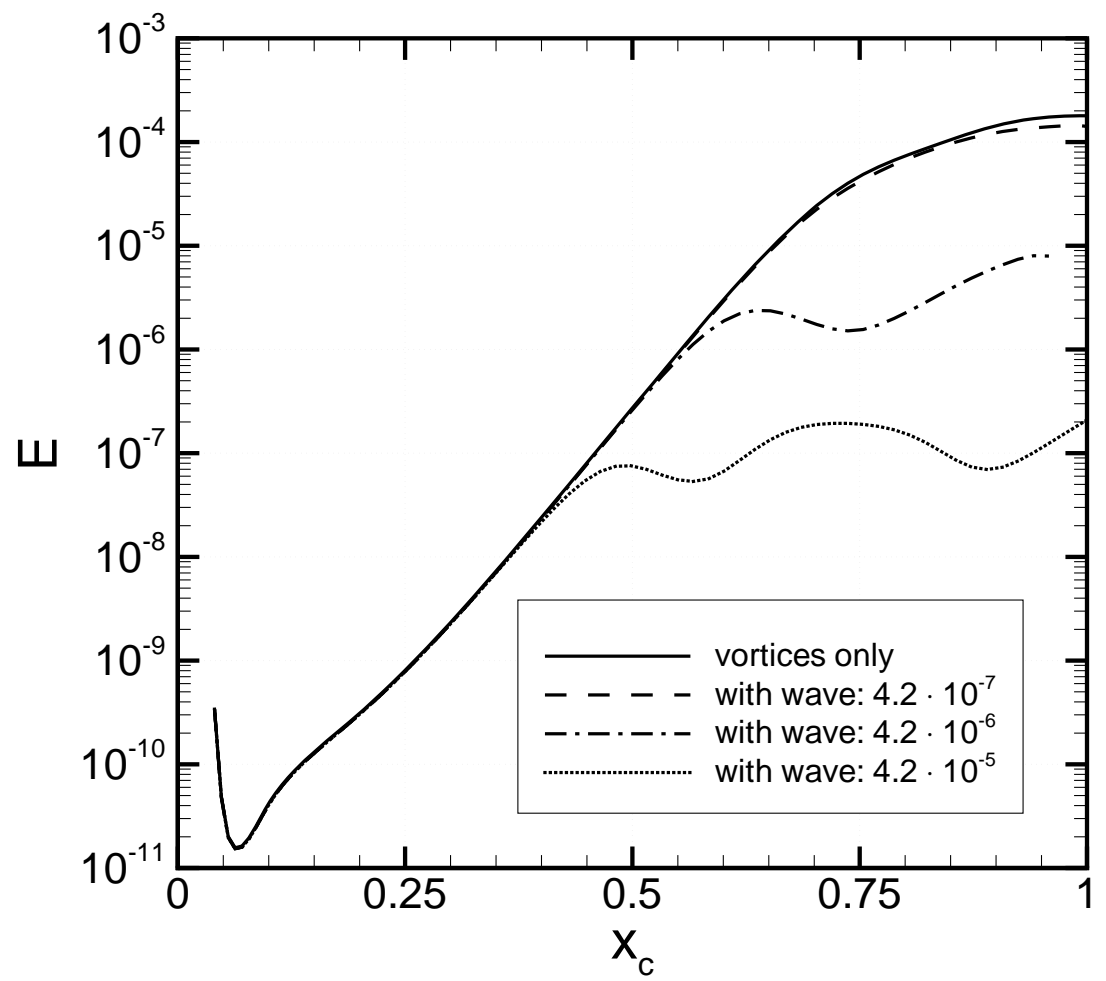

Figure 4.22: Total fluctuation energy $E$ of vortices $(0,-4,1)-(0,-16,4)$ as defined in equation (4.20) from PSE computations for a wave-vortex interaction with different initial amplitudes of the traveling waves.

\subsection{The "Saturated" State}

The discussion of the amplitude evolution of stationary crossflow vortices in section 4.3 led to the observation that changing the initial vortex amplitude does not only affect the extent of the domain, where linear instability theory is valid. It also has significant influence on the nonlinear evolution of the vortices. In particular, high vortex amplitudes at the end of the plate are not 
reached if the vortex is initialized at high amplitude, but if moderate initial amplitudes are specified.

Using quantity $E\left(x_{c}\right)$ as defined in equation (4.20) as measure of vortex amplitude, this phenomenon is illustrated in figure 4.23 for stationary crossflow vortices. The abscissa $E$ is plotted both on a linear scale (top) and on the commonly used semi-log scale (bottom). For the PSE computations (solid lines without symbols), the crossflow vortex $(0,-1,1)$ has been initialized with different amplitudes $\left|\tilde{u}_{c}\right|_{\max }: 7.2 \cdot 10^{-5}, 2.2 \cdot 10^{-4}, 7.2 \cdot 10^{-4}, 2.2 \cdot 10^{-3}, 7.2 \cdot 10^{-3}$, and $2.2 \cdot 10^{-2}$. With an initial vortex amplitude of $7.2 \cdot 10^{-5}$ - this corresponds to the case shown in figure 4.6 - the total fluctuation energy increases monotonically over the whole computational domain and reaches its maximum $E=1.79 \cdot 10^{-4}$ at the end of the plate. If the initial amplitude of the vortex is increased by a factor of three to $2.2 \cdot 10^{-4}$, the maximal fluctuation energy reduces to $1.58 \cdot 10^{-4}$. This value is reached at $x_{c}=0.91$. Further downstream, the fluctuation energy reduces monotonically to $E=1.51 \cdot 10^{-4}$ at the end of the plate. Increasing the initial amplitude further to $7.2 \cdot 10^{-4}$ results in an energy maximum of $1.32 \cdot 10^{-4}$ at $x_{c}=0.86$. The amplitude evolution of the different modes is shown in figure 4.7 for these conditions. At $x_{c}=1.0$ the fluctuation energy is reduced to $1.22 \cdot 10^{-4}$ in this case.

This phenomenon - higher initial vortex amplitudes lead to lower amplitudes at saturation - has been confirmed by Bonfigli \& Kloker [12] using spatial DNS. For the pressure distribution (2.2) and the free-stream parameters given in table 2.1, they computed the spatial evolution of the stationary crossflow vortex and its harmonics for various initial disturbance amplitudes and observed the same effect. Two of their results are included in figure 4.23 (dashed lines). While the general agreement between the results from the spatial DNS and the PSE computations is very good, the direct numerical simulations predict slightly higher maximal disturbance amplitudes than the PSE computations. One reason for these small discrepancies might be a difference in the initialization of the crossflow vortex. In addition, different methods have been used to calculate the laminar base flow for the two approaches. For our PSE computation, the laminar base flow has been computed using first order boundary layer theory, as described in section 2.3. Bonfigli \& Kloker used a spatial DNS code to compute the laminar base flow for their analysis. For the chordwise location $x_{c}=0.6$, the wallparallel velocity components $U_{s}$ and $W_{s}$ as obtained by using these two approaches are compared in figure 4.24. The small differences between the boundary layer profiles might be the cause of the small discrepancies between the PSE and DNS results shown in figure 4.23.

For comparison and in order to get a feeling for the initial amplitudes relevant for the DLR swept plate experiment, the quantity $E\left(x_{c}\right)$ has also been extracted from the PSE results for a stationary crossflow vortex obtained by Bertolotti [8]. These results, included in figure 4.23 as solid line with square symbols, have been obtained for a crossflow vortex generated by a steady 

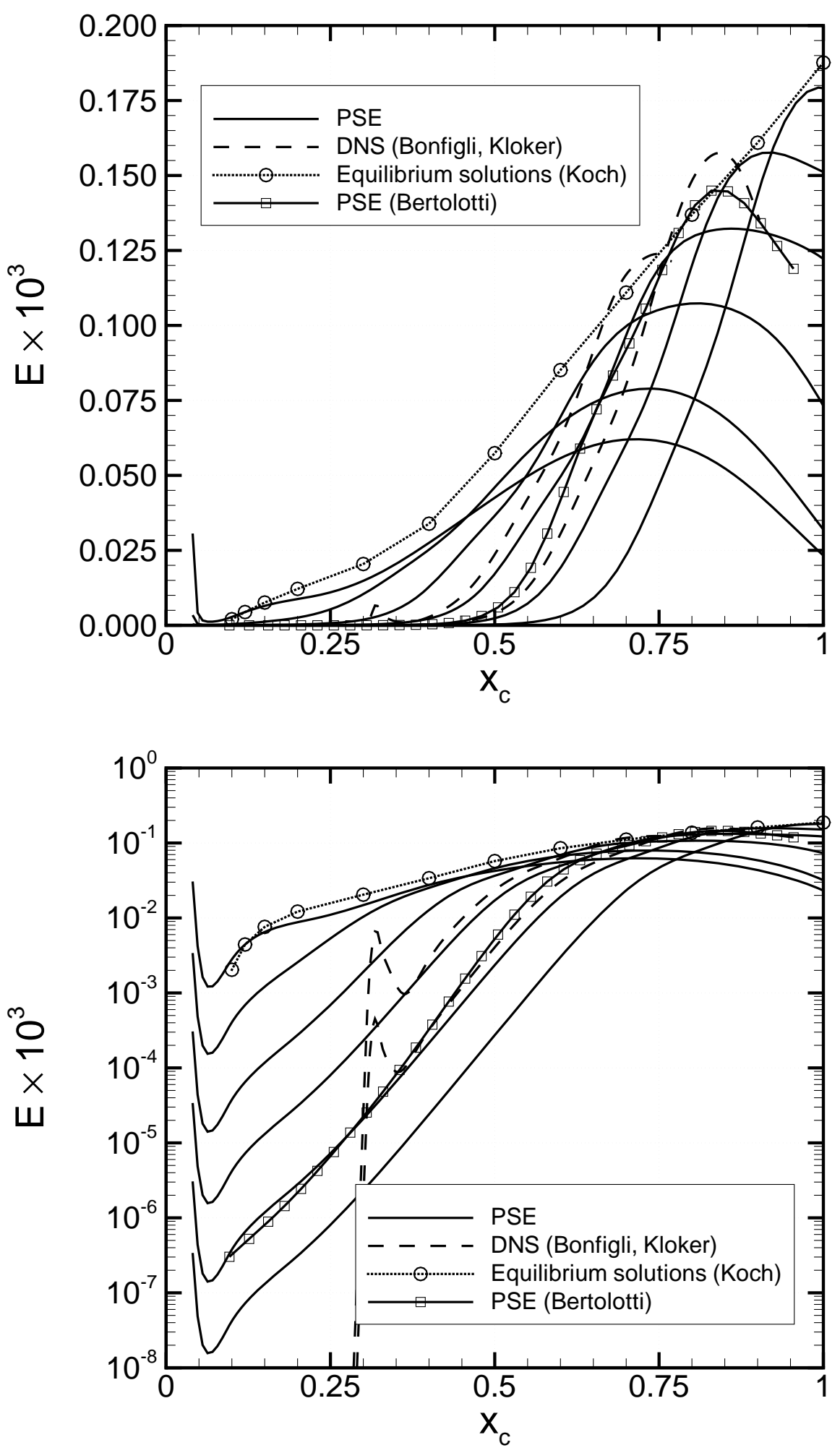

Figure 4.23: Total fluctuation energy $E$ of stationary crossflow vortex with spanwise wave number $\beta_{c}=261.8$ (including the first three harmonics) on linear scale (top) and semi-log scale (bottom). Different lines correspond to different initial amplitudes of the fundamental vortex (DNS and PSE). 

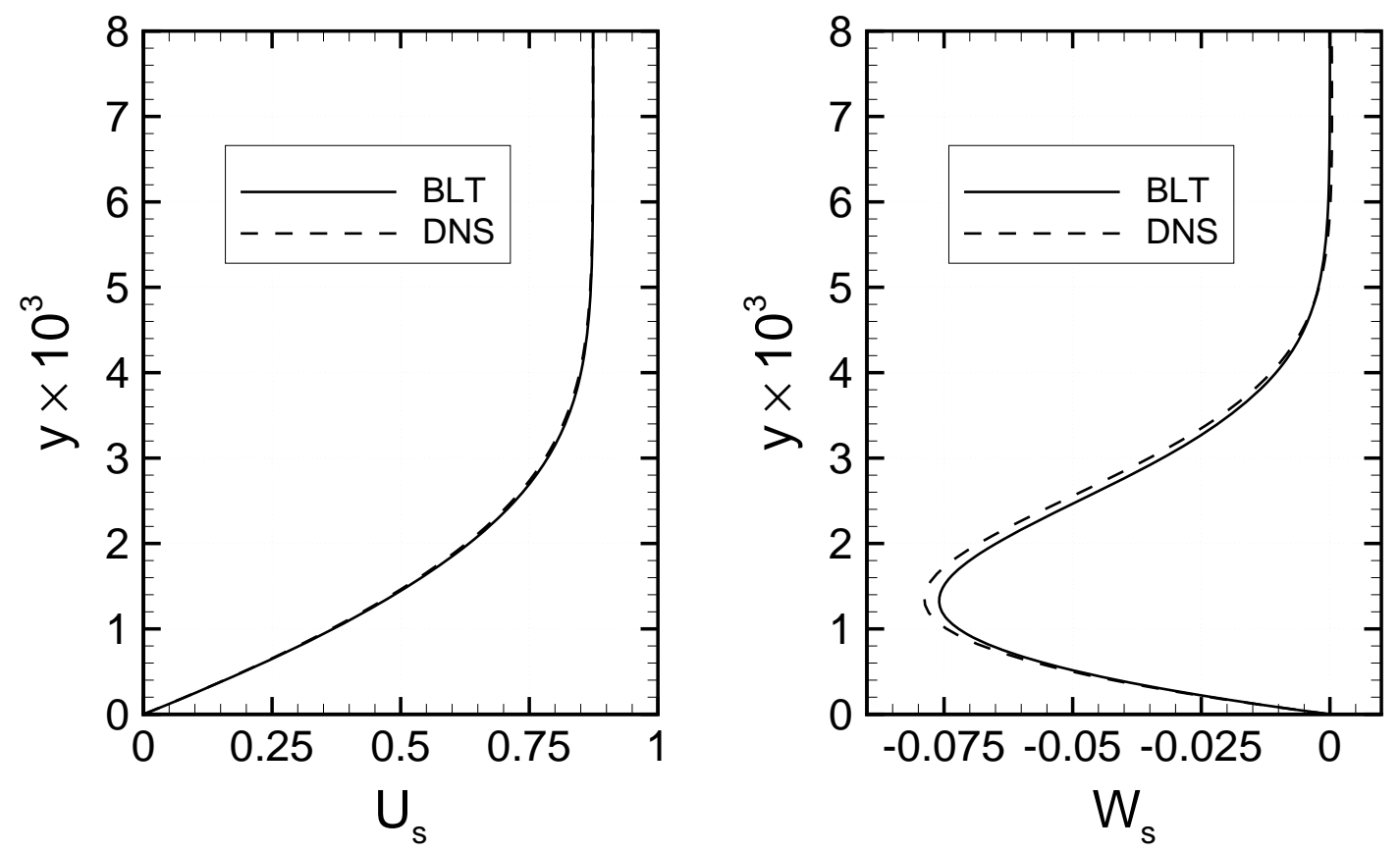

Figure 4.24: Laminar base flow at $x_{c}=0.6$ as computed using first order boundary layer theory (BLT) and spatial direct numerical simulation (DNS). The DNS has been performed by Bonfigli \& Kloker [12].

surface bump at $x_{c}=0.08$. Even though the free stream conditions used by Bertolotti [8] differ slightly from those described in chapter 2, the saturation behavior of the vortices agrees well with that observed in the other studies. A similar oscillatory behavior has been observed in PSE computations by Malik et al. [61] and in solutions obtained by nonlinear critical layer theory (cf. Smith et al. [89], Gajjar [25], Brown \& Smith [15]).

The circles in figure 4.23 denote temporal nonlinear equilibrium solutions for the same laminar base flow and the same parameters of the crossflow vortex as used in the PSE computations. These equilibrium solutions obtained by Koch (cf. Koch [52] for a Falkner-Skan-Cooke similar boundarylayer base flow) are discussed in detail in Koch et al. [53]. In contrast to the PSE results, the nonlinear equilibrium solutions do not depend on the initial amplitude of the disturbance. The temporal equilibrium solutions are computed under the parallel-flow assumption, i.e. the laminar base flow is assumed to be locally independent of $x_{c}$. The wallnormal velocity component $V$ of the laminar base flow is neglected, nonlocal terms are only considered using the similarity assumption. In spite of these substantial differences in the formulation, the fluctuation energy of the temporal equilibrium solutions agrees very well with the envelope of the spatial PSE results.

While the equilibrium solutions (for a specific crossflow vortex) only depend on $x_{c}$, the results of PSE and DNS analysis are not attracted by a unique satu- 
ration curve. They rather indicate an oscillating state. Using temporal DNS, Wagner [94] obtained time-periodic disturbance equilibria for a FalknerSkan-Cooke base flow at Reynolds number $R e_{l o c}=826$, where

$$
R e_{l o c}=\frac{Q_{e}^{*} d^{*}}{\nu^{*}}
$$

with

$$
Q_{e}^{*}=Q_{\infty}^{*} \sqrt{\sin ^{2} \phi_{\infty}+\left(1-c_{p}\right) \cos ^{2} \phi_{\infty}}
$$

and

$$
d^{*}=\sqrt{\frac{\nu^{*} x_{c}^{*}}{U_{c, e}^{*}}} .
$$

$U_{c, e}^{*}$ is the chordwise velocity component at the boundary layer edge as defined in equation 2.5. With the free stream parameters used in the PSE analysis, $R e_{l o c}=826$ corresponds to $x_{c} \approx 0.8$. For $R e_{l o c}=260$ and base flow parameters similar to those used by Wagner [94], Meyer [63] observed stationary equilibria in his temporal numerical simulations. A comparison between these results indicates a spatial Hopf bifurcation of the stationary solution. This explanation is in agreement with the results of Koch [51], who studied equilibrium solutions of Tollmien-Schlichting waves in Blasius boundary layer flow. In an investigation of the nonlinear instability of stationary crossflow vortices, Gajjar [25] predicted nonlinear amplitude oscillation in threedimensional boundary layer flow using asymptotic expansion methods. In the light of these results, the decrease of disturbance energy discussed above points to the fact that there exists a critical Reynolds number corresponding to a chordwise location on the plate, downstream of which a saturated crossflow vortex does not represent a stable solution to the governing equations.

In order to show this more clearly, the oscillatory behavior of the vortex amplitude has to be separated from the spatial development of the boundary layer. This is achieved by artificially freezing the laminar base flow as follows: For different freezing locations $x_{c \text {,freezer }}$ the evolution of a crossflow vortex with spanwise wave number $\beta_{c}=261.8$ is studied using the PSE marching procedure without updating the laminar base flow profiles. For this procedure, the complete undisturbed base flow profiles are considered, i.e. wallnormal velocity component and streamwise derivatives are not neglected. Even though the influence of the nonparallel terms on the results of this pseudo-marching turns out to be small, one has to keep in mind that this flow can not be realized in a laboratory. However, it proves to be suitable to substantiate the spatial instability of the saturated stationary vortex. Results of the pseudo-marching for different freezing locations are shown in figure 4.25 .

In the front part of the plate - at $x_{c, \text { freeze }}=0.1$ - the crossflow vortex grows exponentially if it is initialized with a small amplitude in the frozen base flow. By nonlinear forcing, a nonlinear mean flow distortion and higher harmonics of the vortex are generated. After this transient phase, the vortex 


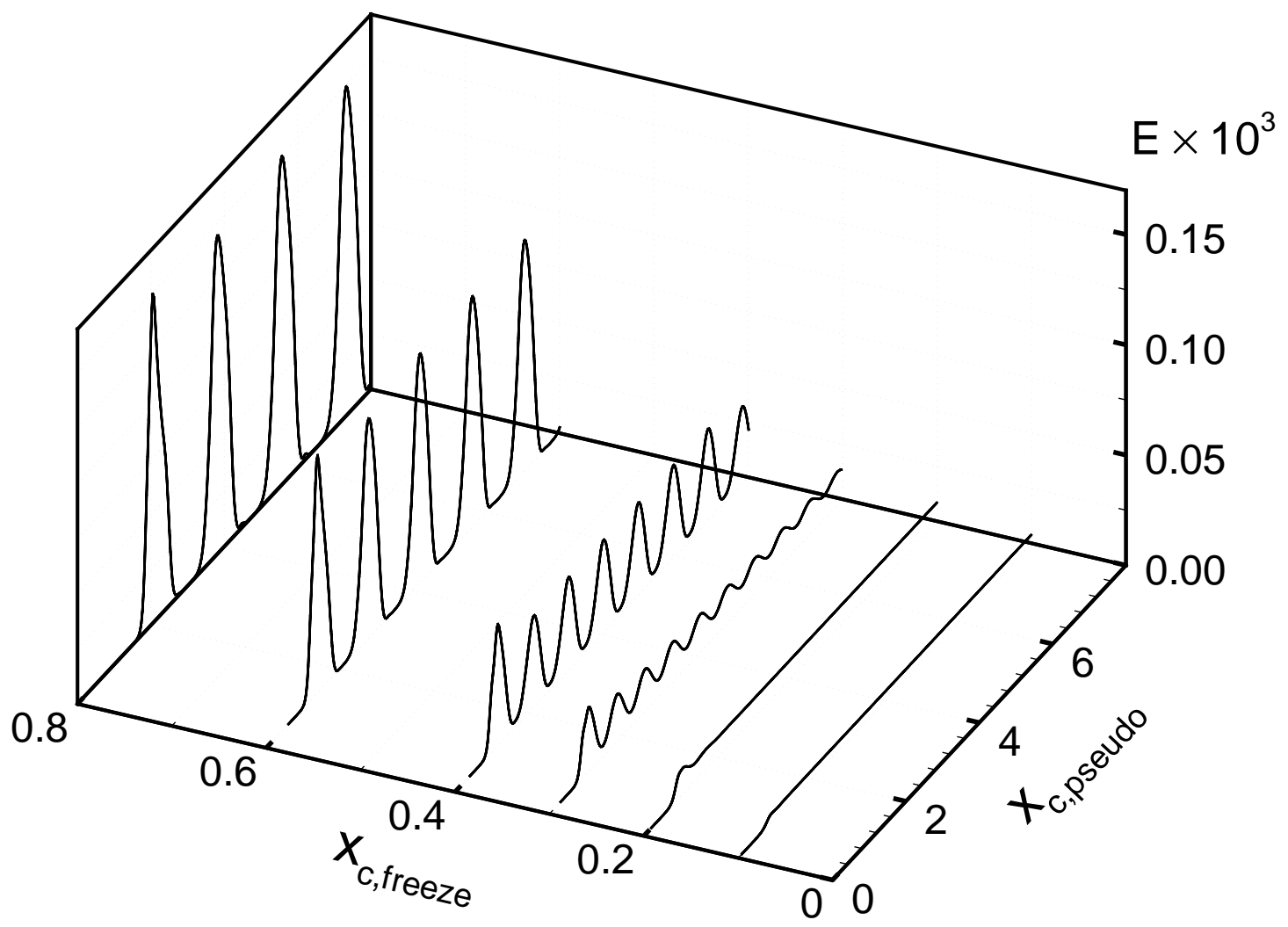

Figure 4.25: Total fluctuation energy $E$ of stationary crossflow vortex with spanwise wave number $\beta_{c}=261.8$ (including the first three harmonics) for different freezing locations $x_{c, \text { freeze }}$ of the laminar base flow. Without updating the laminar base flow profiles the PSE are integrated along the pseudomarching coordinate $x_{c, p s e u d o}$.

reaches a unique saturation amplitude which does not vary with the pseudomarching coordinate $x_{c, p s e u d o}$. A very similar behavior can be observed for $x_{c, \text { freeze }}=0.2$. The saturation amplitude is independent of the initial conditions of the vortex as can be seen from figure 4.26 (top), where two computations with different initial amplitudes of the vortex are compared. As expected, a lower initial amplitude only extends the region with transient behavior to higher values of $x_{c, p s e u d o}$, but it has no influence on the saturated equilibrium state.

At $x_{c, \text { freeze }}=0.3$, the region with transient behavior extends to very high values of $x_{x, p s e u d o}$. The total fluctuation energy $E$ of the vortex still exhibits a damped oscillation. For sufficiently high values of $x_{c, p s e u d o}$ a unique saturation amplitude can be expected. However, the low decay rate of the oscillation indicates that this location is not far upstream of a bifurcation point, where the saturated crossflow vortex becomes spatially unstable. Downstream of this spatial Hopf bifurcation, stationary solutions with oscillatory behavior in space exist as can be seen from the oscillation of fluctuation en- 

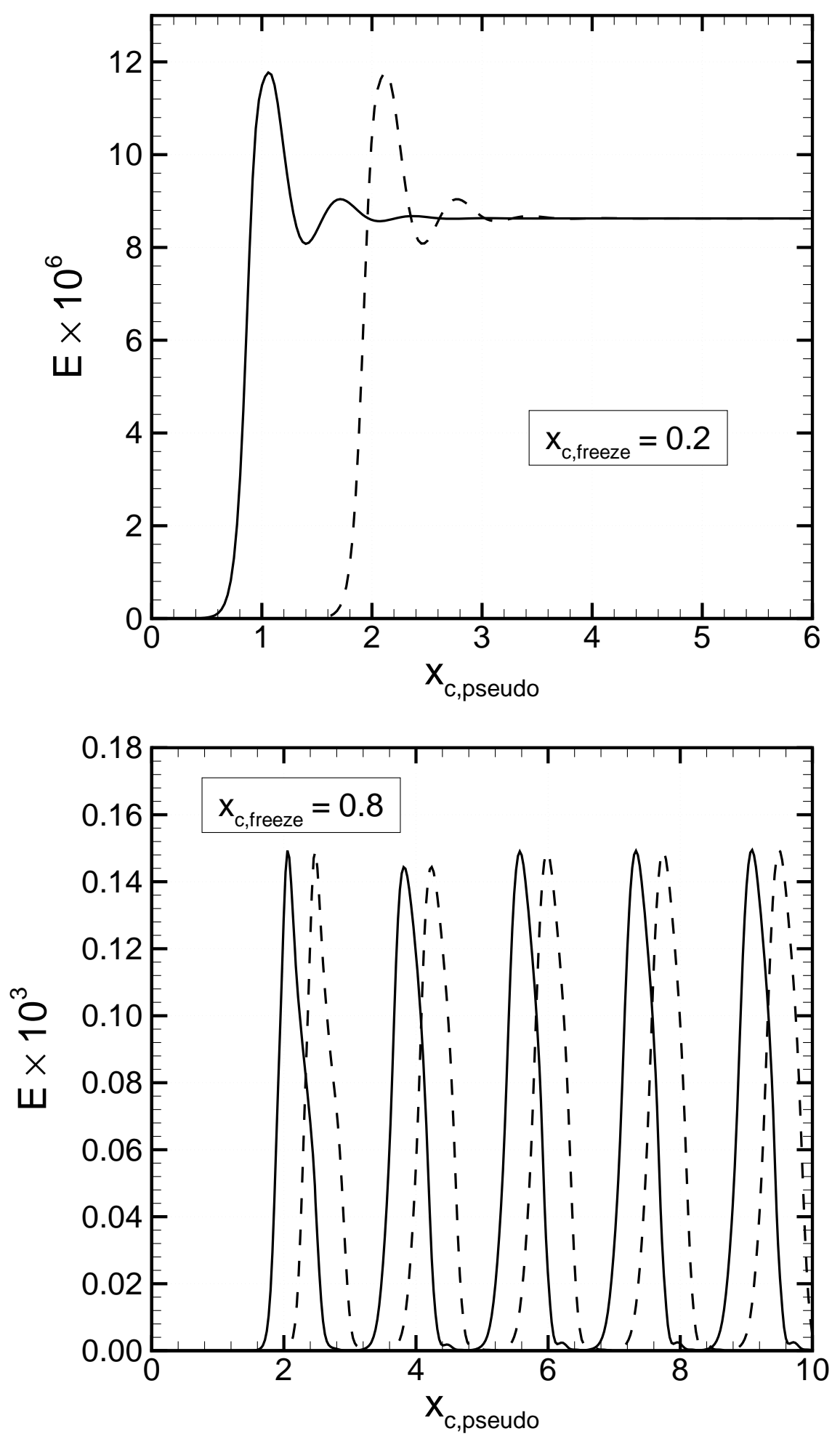

Figure 4.26: Total fluctuation energy $E$ of stationary crossflow vortex with spanwise wave number $\beta_{c}=261.8$ (including the first three harmonics) for $x_{c, \text { freeze }}=0.2$ (top) and $x_{c, \text { freeze }}=0.8$ (bottom). In the computations shown by the dashed lines the initial amplitude of the vortex is lower than in the computations shown by the solid lines. 
ergy at all freezing locations downstream of $x_{c, \text { freeze }}=0.3$. Figure 4.26 (bottom) shows that this oscillatory state is independent of the initial conditions of the vortex in the sense that a change in initial amplitude only leads to a "phase shift" of the nonlinearly oscillating fluctuation energy $E$ as function of $x_{c, p s e u d o}$. The wave length of this oscillation is of the order of the chord length of the plate. For $x_{c, \text { freeze }}=0.8$, one oscillation cycle covers a spatial domain corresponding to about twice the length of the test plate. This fact makes it difficult to verify the bifurcation experimentally in a spatially evolving base flow. However, the observation that a unique saturation state does not exist calls for careful interpretation of numerical results regarding disturbance saturation and secondary instability. Furthermore, it emphasizes the central role of initial conditions (i.e. receptivity) in transition scenarios.

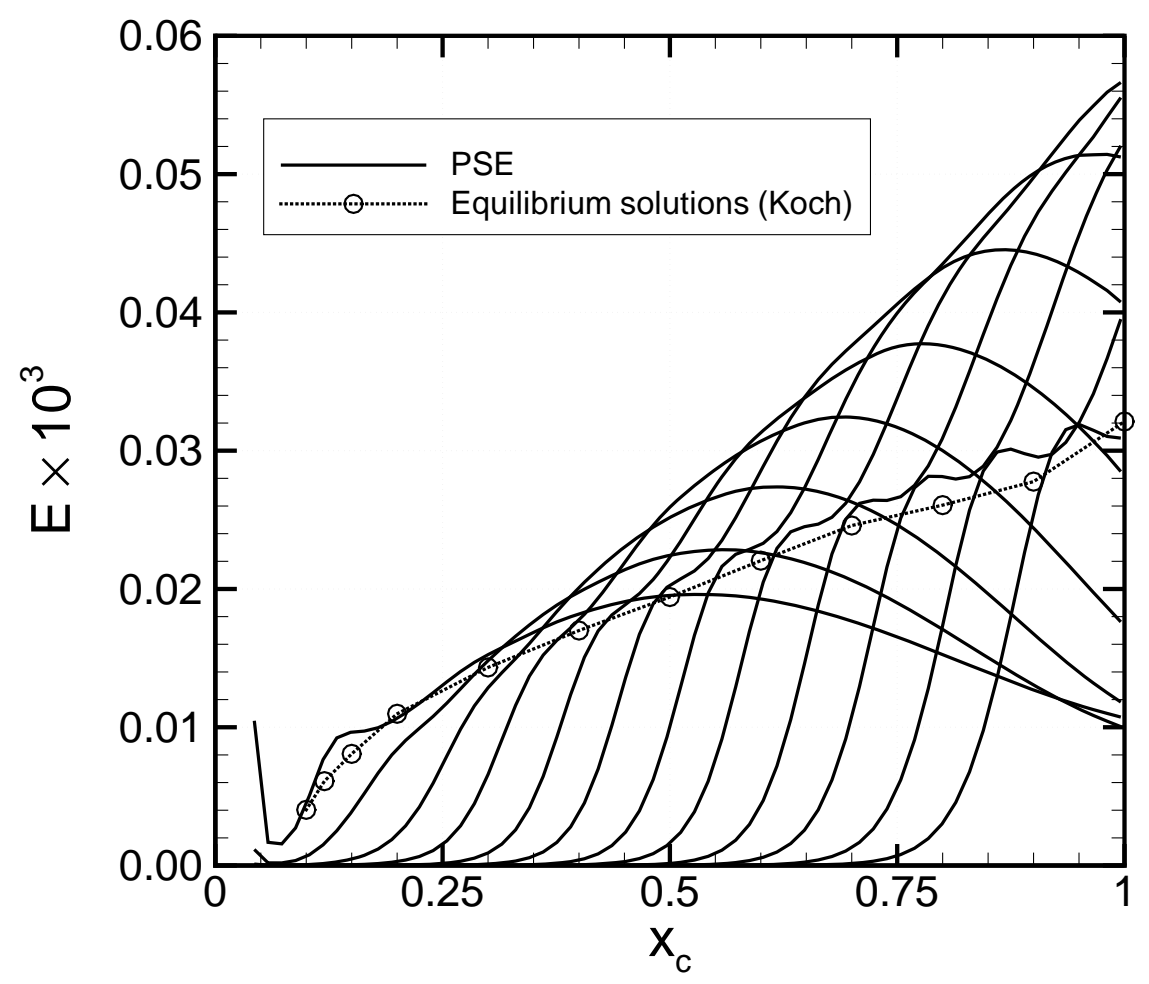

Figure 4.27: Total fluctuation energy $E$ of traveling crossflow wave with spanwise wave number $\beta_{c}=261.8$ and frequency $f=3.553$ (including the first three harmonics). Different solid lines correspond to different initial amplitudes of the fundamental wave in the PSE computations.

In the remainder of this section, the bifurcation leading to spatially oscillating solutions is discussed for traveling disturbances in the three-dimensional boundary layer flow of the DLR swept plate experiment. Figure 4.27 shows the evolution of total fluctuation energy $E$ for a traveling wave with spanwise wave number $\beta_{c}=261.8$ and frequency $f=3.553$ (this corresponds to a 
dimensional frequency $f^{*}=135 \mathrm{~Hz}$ ) including its first three harmonics. The PSE computations are performed with the initial amplitude $\left|\tilde{u}_{c}\right|_{\max }$ of the fundamental wave varying between $9.6 \cdot 10^{-8}$ and $2.8 \cdot 10^{-2}$. If the wave is initialized at very low amplitude it does not reach its maximal amplitude by the end of the plate. As observed for the stationary vortex, the highest disturbance amplitudes are reached if the wave is initialized with moderate amplitudes. For the wave with spanwise wave number $\beta_{c}=261.8$ and frequency $f=3.553$, an initial amplitude $\left|\tilde{u}_{c}\right|_{\max }=9.6 \cdot 10^{-6}$ at the neutral point of the disturbance leads to the highest maximal fluctuation energy $E=5.7 \cdot 10^{-5}$ at the end of the plate. The amplitude of the fundamental mode reaches $\left|\tilde{u}_{c}\right|_{\max }=17.5 \%$ at $x_{c}=1.0$ for these initial conditions. If the initial amplitude of the wave is further increased, the location where the fluctuation energy $E$ reaches its maximum moves upstream, and the maximal fluctuation energy decreases. As for the stationary vortex, no unique saturation curve can be observed for the amplitude of the wave. In contrast to the behavior of the stationary vortex, the amplitude (i.e. the total fluctuation energy) of the traveling wave does not in all cases increase monotonically until it reaches its global maximum. It rather hesitates at some intermediate value, before it continues to grow to the global maximum. This intermediate value of total fluctuation energy depends on the chordwise location $x_{c}$ and agrees well with the fluctuation energy of temporal nonlinear equilibrium solutions computed by Koch for the same wave (see Koch et al. [53]).

This behavior points to an equilibrium solution, which - even though it is probably spatially unstable - has some influence on the amplitude evolution of the traveling wave. As for the stationary vortex, a spatial Hopf bifurcation downstream of $x_{c}=0.3$ is substantiated by investigating the amplitude evolution of the wave in a PSE pseudo-marching. For different freezing locations $x_{c, \text { freezer }}$ this evolution is summarized in figure 4.28 . The influence of the unstable equilibrium solution on the nonlinearly oscillating solution downstream of the bifurcation point can not only be observed in the transient phase of the the spatial oscillation. As can be seen from figure 4.29, this influence continues to exist in the region of periodic nonlinear oscillation. 


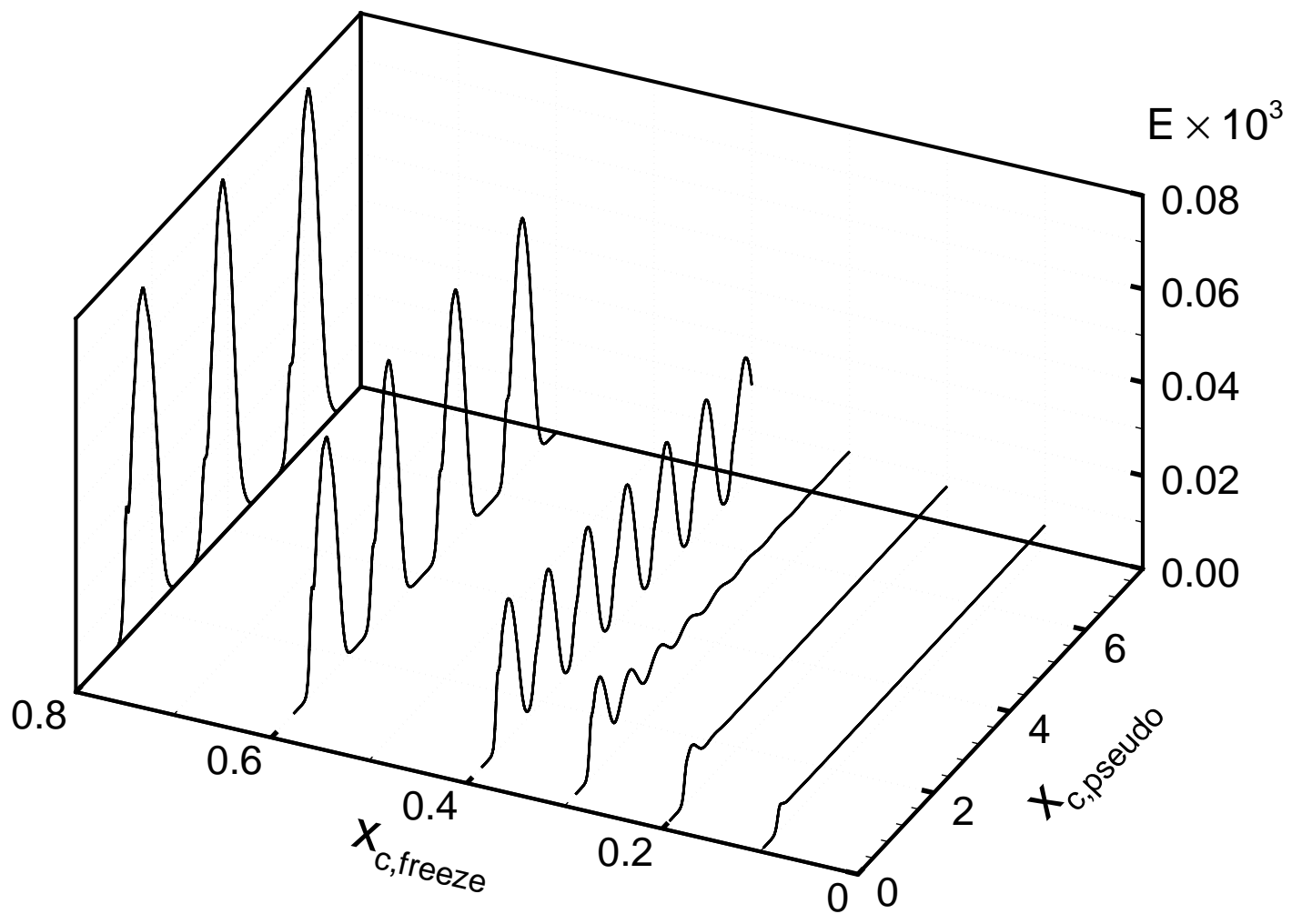

Figure 4.28: Total fluctuation energy $E$ of traveling crossflow wave with spanwise wave number $\beta_{c}=261.8$ and frequency $f=3.553$ (including the first three harmonics) for different freezing locations $x_{c, \text { freeze }}$ of the laminar base flow. Without updating the laminar base flow profiles the PSE are integrated along the pseudo-marching coordinate $x_{c, p s e u d o}$. 


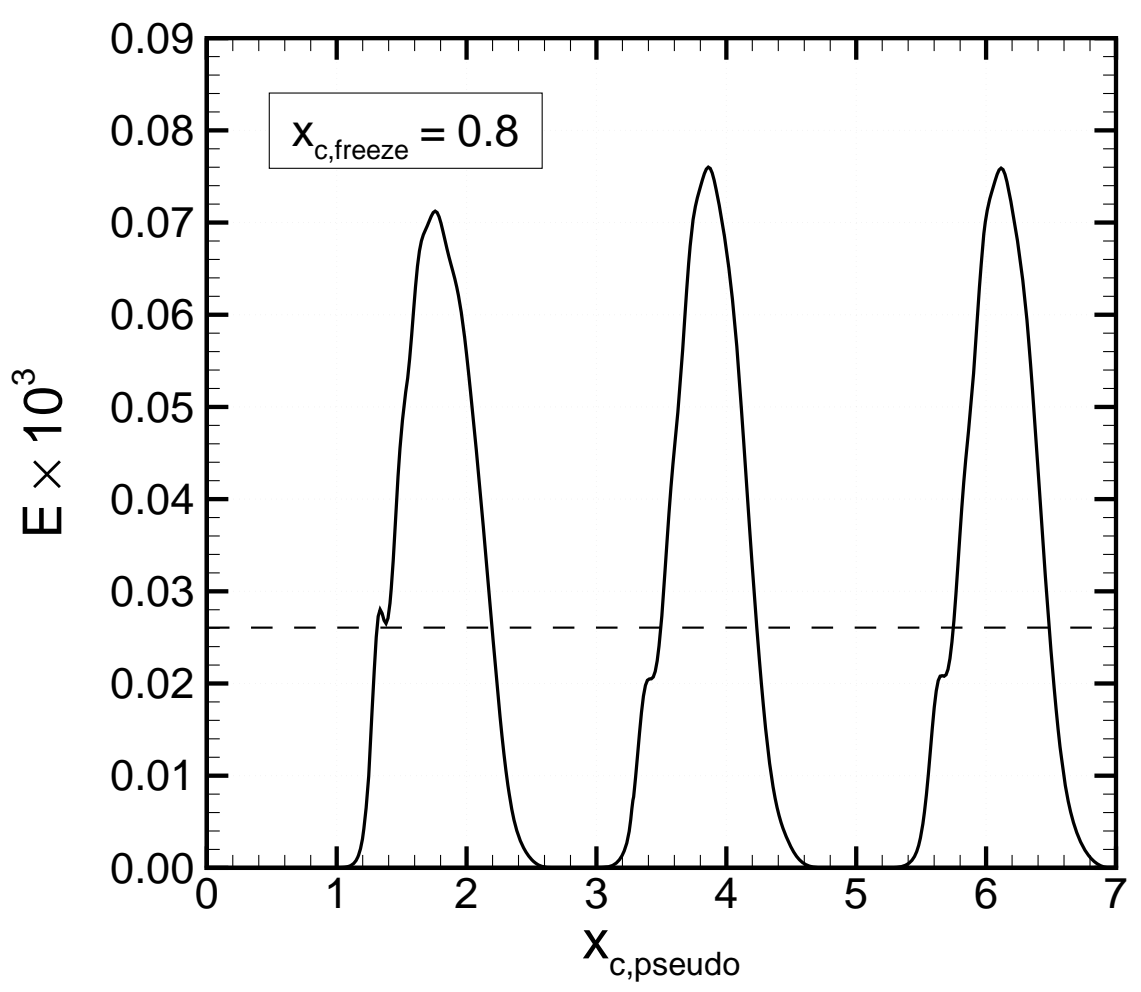

Figure 4.29: Total fluctuation energy $E$ of traveling crossflow wave with spanwise wave number $\beta_{c}=261.8$ and frequency $f=3.553$ (including the first three harmonics) from PSE computation for $x_{c, \text { freeze }}=0.8$. The dashed line indicates the fluctuation energy of the corresponding temporal equilibrium solution. 


\section{Chapter 5}

\section{Secondary Instability Analysis and Laminar-Turbulent Transition}

A linear temporal secondary instability analysis of the primarily distorted flow field reveals the dependence of secondary, high-frequency instability on the amplitude of the primary vortices at significantly lower computational costs than a direct investigation using the PSE, which at present is not possible with state of the art desktop computers.

In the limit of low primary vortex amplitude, the low-frequency waves corresponding to traveling primary crossflow waves are recovered. It is shown that, in the frame of Floquet theory, secondary disturbances can propagate only in direction of the primary vortex axis. With increasing amplitude of the primary vortex, more and more unstable secondary disturbances are found. If the primary vortex amplitude is higher than $10 \%$ of the free-stream velocity, high-frequency secondary disturbances located on the back of the primary vortices, where both spanwise and wallnormal shear of the distorted base flow exhibit maxima, by far exceed all other modes in growth and can therefore be expected to play a central role in the transition process.

\subsection{Introduction}

In this chapter, a temporal secondary instability analysis of stationary crossflow vortices is presented. High-resolution nonlinear PSE results, as presented in chapter 4 (cf. figure 4.11), are used as primarily distorted base flow. The secondary analysis is performed using Floquet theory (cf. Herbert [38]). After a discussion of the theoretical background and the numerical solution procedure in the following two sections, the growth rates and structures of secondary disturbances are studied in section 5.4. There, particular attention is directed to high-frequency secondary disturbances. 


\subsection{Secondary Instability Theory}

In a coordinate system oriented in the direction of the primary crossflow mode (see figure 2.3) and moving with the propagation speed $C$ of the primary disturbance in $x_{\psi}$-direction

$$
\left(\begin{array}{c}
x_{\psi} \\
y \\
z_{\psi} \\
t
\end{array}\right)=\left(\begin{array}{cccc}
\cos \psi_{c} & 0 & \sin \psi_{c} & -C \\
0 & 1 & 0 & 0 \\
-\sin \psi_{c} & 0 & \cos \psi_{c} & 0 \\
0 & 0 & 0 & 1
\end{array}\right)\left(\begin{array}{c}
x_{c} \\
y \\
z_{c} \\
t
\end{array}\right)
$$

the secondary instability equations are derived from the conservation equations for momentum and mass

$$
\begin{aligned}
\frac{\partial u_{\psi}}{\partial t}-C \frac{\partial u_{\psi}}{\partial x_{\psi}}+u_{\psi} \frac{\partial u_{\psi}}{\partial x_{\psi}}+v \frac{\partial u_{\psi}}{\partial y}+w_{\psi} \frac{\partial u_{\psi}}{\partial z_{\psi}} & =-\frac{\partial p}{\partial x_{\psi}}+\frac{1}{R e} \Delta_{\psi} u_{\psi} \\
\frac{\partial v}{\partial t}-C \frac{\partial v}{\partial x_{\psi}}+u_{\psi} \frac{\partial v}{\partial x_{\psi}}+v \frac{\partial v}{\partial y}+w_{\psi} \frac{\partial v}{\partial z_{\psi}} & =-\frac{\partial p}{\partial y}+\frac{1}{R e} \Delta_{\psi} v \\
\frac{\partial w_{\psi}}{\partial t}-C \frac{\partial w_{\psi}}{\partial x_{\psi}}+u_{\psi} \frac{\partial w_{\psi}}{\partial x_{\psi}}+v \frac{\partial w_{\psi}}{\partial y}+w_{\psi} \frac{\partial w_{\psi}}{\partial z_{\psi}} & =-\frac{\partial p}{\partial z_{\psi}}+\frac{1}{R e} \Delta_{\psi} w_{\psi} \\
\frac{\partial u_{\psi}}{\partial x_{\psi}}+\frac{\partial v}{\partial y}+\frac{\partial w_{\psi}}{\partial z_{\psi}} & =0
\end{aligned}
$$

where

$$
\Delta_{\psi}=\frac{\partial^{2}}{\partial x_{\psi}^{2}}+\frac{\partial^{2}}{\partial y^{2}}+\frac{\partial^{2}}{\partial z_{\psi}^{2}}
$$

and

$$
R e=\frac{Q_{\infty}^{*} c^{*}}{\nu^{*}} .
$$

For stationary primary disturbances, $C=0$.

The total flow field $\mathbf{q}_{\psi}=\left(u_{\psi}, v, w_{\psi}, p\right)^{T}$ is decomposed in the following way:

$$
\mathbf{q}_{\psi}=\mathbf{Q}_{\psi}+\tilde{\mathbf{q}}_{\psi}+\overline{\mathbf{q}}_{\psi}
$$

Following closely the derivation presented in Koch et al. [53], decomposition (5.8) is introduced into equations (5.2) - (5.5), and the equations satisfied by the primarily disturbed flow field are subtracted. The steady laminar base flow $\mathbf{Q}_{\psi}$ is then - in contrast to the investigations in the previous chapters assumed to be quasi-parallel:

$$
\mathbf{Q}_{\psi}(y)=\left(U_{\psi}(y), 0, W_{\psi}(y), P\right)^{T} .
$$


Neglecting nonlocal terms of the nonlinear PSE solution, the primary disturbance flow field $\tilde{\mathbf{q}}_{\psi}$ can be written in the form

$$
\tilde{\mathbf{q}}_{\psi}\left(x_{\psi}, y\right)=\left(\begin{array}{c}
\tilde{u}_{\psi}\left(x_{\psi}, y\right) \\
\tilde{v}\left(x_{\psi}, y\right) \\
\tilde{w}_{\psi}\left(x_{\psi}, y\right) \\
\tilde{p}\left(x_{\psi}, y\right)
\end{array}\right)=\sum_{m=-N_{p}}^{N_{p}}\left(\begin{array}{c}
\hat{u}_{\psi, m}(y) \\
\hat{v}_{m}(y) \\
\hat{w}_{\psi, m}(y) \\
\hat{p}_{m}(y)
\end{array}\right) e^{i m \alpha_{\psi} x_{\psi}}
$$

i.e. the primarily distorted base flow is spatially periodic in $x_{\psi}$-direction with period

$$
\lambda_{x_{\psi}}=\frac{2 \pi}{\alpha_{\psi}}=\frac{2 \pi}{\sqrt{\alpha_{c, r, 0}^{2}+\beta_{c}^{2}}} .
$$

For small values of the weight $\sigma$, the generalized auxiliary condition (4.9) takes care that the contribution of the amplitude function to the physical wave number (cf. equation (3.29)) is small. This justifies the choice of the real part of the chordwise wave number of the fundamental mode $\alpha_{c, r, 0}$ in equation (5.11). In equation (5.8), $\overline{\mathbf{q}}_{\psi}$ denotes secondary disturbances.

To eliminate the pressure terms from the resulting secondary instability equations

$$
\begin{gathered}
\frac{\partial \bar{u}_{\psi}}{\partial t}-C \frac{\partial \bar{u}_{\psi}}{\partial x_{\psi}}+\bar{u}_{\psi} \frac{\partial \tilde{u}_{\psi}}{\partial x_{\psi}}+U_{\psi} \frac{\partial \bar{u}_{\psi}}{\partial x_{\psi}}+\tilde{u}_{\psi} \frac{\partial \bar{u}_{\psi}}{\partial x_{\psi}}+\bar{v} \frac{d U_{\psi}}{d y}+\bar{v} \frac{\partial \tilde{u}_{\psi}}{\partial y}+\tilde{v} \frac{\partial \bar{u}_{\psi}}{\partial y} \\
+W_{\psi} \frac{\partial \bar{u}_{\psi}}{\partial z_{\psi}}+\tilde{w}_{\psi} \frac{\partial \bar{u}_{\psi}}{\partial z_{\psi}}+\frac{\partial \bar{p}}{\partial x_{\psi}}-\frac{1}{R e} \Delta_{\psi} \bar{u}_{\psi}=0 \\
\frac{\partial \bar{v}}{\partial t}-C \frac{\partial \bar{v}}{\partial x_{\psi}}+\bar{u}_{\psi} \frac{\partial \tilde{v}}{\partial x_{\psi}}+U_{\psi} \frac{\partial \bar{v}}{\partial x_{\psi}}+\tilde{u}_{\psi} \frac{\partial \bar{v}}{\partial x_{\psi}}+\bar{v} \frac{\partial \tilde{v}}{\partial y}+\tilde{v} \frac{\partial \bar{v}}{\partial y} \\
+W_{\psi} \frac{\partial \bar{v}}{\partial z_{\psi}}+\tilde{w}_{\psi} \frac{\partial \bar{v}}{\partial z_{\psi}}+\frac{\partial \bar{p}}{\partial y}-\frac{1}{R e} \Delta_{\psi} \bar{v}=0 \\
\frac{\partial \bar{w}_{\psi}}{\partial t}-C \frac{\partial \bar{w}_{\psi}}{\partial x_{\psi}}+\bar{u}_{\psi} \frac{\partial \tilde{w}_{\psi}}{\partial x_{\psi}}+U_{\psi} \frac{\partial \bar{w}_{\psi}}{\partial x_{\psi}}+\tilde{u}_{\psi} \frac{\partial \bar{w}_{\psi}}{\partial x_{\psi}}+\bar{v} \frac{d W_{\psi}}{d y}+\bar{v} \frac{\partial \tilde{w}_{\psi}}{\partial y}+\tilde{v} \frac{\partial \bar{w}_{\psi}}{\partial y} \\
+W_{\psi} \frac{\partial \bar{w}_{\psi}}{\partial z_{\psi}}+\tilde{w}_{\psi} \frac{\partial \bar{w}_{\psi}}{\partial z_{\psi}}+\frac{\partial \bar{p}}{\partial z_{\psi}}-\frac{1}{R e} \Delta_{\psi} \bar{w}_{\psi}=0 \\
\quad \frac{\partial \bar{u}_{\psi}}{\partial x_{\psi}}+\frac{\partial \bar{v}}{\partial y}+\frac{\partial \bar{w}_{\psi}}{\partial z_{\psi}}=0
\end{gathered}
$$

curl is applied to (5.12) - (5.14) yielding

$$
\begin{gathered}
\left\{\frac{\partial}{\partial t}+\left(U_{\psi}-C\right) \frac{\partial}{\partial x_{\psi}}+W_{\psi} \frac{\partial}{\partial z_{\psi}}-\frac{1}{R e} \Delta_{\psi}\right\} \bar{\xi}_{\psi}+\frac{d U_{\psi}}{d y} \frac{\partial \bar{w}_{\psi}}{\partial x_{\psi}}-\frac{d W_{\psi}}{d y} \frac{\partial \bar{u}_{\psi}}{\partial x_{\psi}}+\frac{d^{2} W_{\psi}}{d y^{2}} \bar{v} \\
+\tilde{u}_{\psi} \frac{\partial \bar{\xi}_{\psi}}{\partial x_{\psi}}+\tilde{v} \frac{\partial \bar{\xi}_{\psi}}{\partial y}+\tilde{w}_{\psi} \frac{\partial \bar{\xi}_{\psi}}{\partial z_{\psi}}+\frac{\partial \tilde{u}_{\psi}}{\partial y} \frac{\partial \bar{w}_{\psi}}{\partial x_{\psi}}-\frac{\partial \tilde{v}}{\partial x_{\psi}} \frac{\partial \bar{u}_{\psi}}{\partial z_{\psi}}+\frac{\partial \tilde{v}}{\partial y} \bar{\xi}_{\psi}
\end{gathered}
$$




$$
\begin{gathered}
+\frac{\partial \tilde{w}_{\psi}}{\partial x_{\psi}} \frac{\partial \bar{u}_{\psi}}{\partial y}-\frac{\partial \tilde{w}_{\psi}}{\partial y} \frac{\partial \bar{u}_{\psi}}{\partial x_{\psi}}+\frac{\partial^{2} \tilde{w}_{\psi}}{\partial x_{\psi} \partial y} \bar{u}_{\psi}+\frac{\partial^{2} \tilde{w}_{\psi}}{\partial y^{2}} \bar{v}=0 \\
\left\{\frac{\partial}{\partial t}+\left(U_{\psi}-C\right) \frac{\partial}{\partial x_{\psi}}+W_{\psi} \frac{\partial}{\partial z_{\psi}}-\frac{1}{R e} \Delta_{\psi}\right\} \bar{\eta}+\frac{d U_{\psi}}{d y} \frac{\partial \bar{v}}{\partial z_{\psi}}-\frac{d W_{\psi}}{d y} \frac{\partial \bar{v}}{\partial x_{\psi}} \\
+\tilde{u}_{\psi} \frac{\partial \bar{\eta}}{\partial x_{\psi}}+\tilde{v} \frac{\partial \bar{\eta}}{\partial y}+\tilde{w}_{\psi} \frac{\partial \bar{\eta}}{\partial z_{\psi}}+\frac{\partial \tilde{u}_{\psi}}{\partial x_{\psi}} \bar{\eta}+\frac{\partial \tilde{u}_{\psi}}{\partial y} \frac{\partial \bar{v}}{\partial z_{\psi}}-\frac{\partial \tilde{v}}{\partial x_{\psi}} \frac{\partial \bar{w}_{\psi}}{\partial y} \\
+\frac{\partial \tilde{w}_{\psi}}{\partial x_{\psi}} \frac{\partial \bar{v}}{\partial y}-\frac{\partial \tilde{w}_{\psi}}{\partial y} \frac{\partial \bar{v}}{\partial x_{\psi}}-\frac{\partial^{2} \tilde{w}_{\psi}}{\partial x_{\psi}^{2}} \bar{u}_{\psi}-\frac{\partial^{2} \tilde{w}_{\psi}}{\partial x_{\psi} \partial y} \bar{v}=0 \\
\left\{\frac{\partial}{\partial t}+\left(U_{\psi}-C\right) \frac{\partial}{\partial x_{\psi}}+W_{\psi} \frac{\partial}{\partial z_{\psi}}-\frac{1}{R e} \Delta_{\psi}\right\} \bar{\zeta}_{\psi}+\frac{d U_{\psi}}{d y} \frac{\partial \bar{w}_{\psi}}{\partial z_{\psi}}-\frac{d^{2} U_{\psi}}{d y^{2}} \bar{v}-\frac{d W_{\psi}}{d y} \frac{\partial \bar{u}_{\psi}}{\partial z_{\psi}} \\
+\tilde{u}_{\psi} \frac{\partial \bar{\zeta}_{\psi}}{\partial x_{\psi}}+\tilde{v} \frac{\partial \bar{\zeta}_{\psi}}{\partial y}+\tilde{w}_{\psi} \frac{\partial \bar{\zeta}_{\psi}}{\partial z_{\psi}}+\frac{\partial \tilde{u}_{\psi}}{\partial y} \frac{\partial \bar{w}_{\psi}}{\partial z_{\psi}}-\frac{\partial \tilde{v}}{\partial x_{\psi}} \frac{\partial \bar{w}_{\psi}}{\partial z_{\psi}}+\frac{\partial \tilde{w}_{\psi}}{\partial x_{\psi}} \frac{\partial \bar{v}}{\partial z_{\psi}} \\
-\frac{\partial \tilde{w}_{\psi}}{\partial y} \frac{\partial \bar{u}_{\psi}}{\partial z_{\psi}}-\frac{\partial^{2} \tilde{u}_{\psi}}{\partial x_{\psi} \partial y} \bar{u}_{\psi}-\frac{\partial^{2} \tilde{u}_{\psi}}{\partial y^{2}} \bar{v}+\frac{\partial^{2} \tilde{v}}{\partial x_{\psi}^{2}} \bar{u}_{\psi}+\frac{\partial^{2} \tilde{v}}{\partial x_{\psi} \partial y} \bar{v}=0
\end{gathered}
$$

where the secondary disturbance vorticity vector is defined as

$$
\left(\begin{array}{c}
\bar{\xi}_{\psi} \\
\bar{\eta} \\
\bar{\zeta}_{\psi}
\end{array}\right)=\left(\begin{array}{c}
\frac{\partial \bar{w}_{\psi}}{\partial y}-\frac{\partial \bar{v}}{\partial z_{\psi}} \\
\frac{\partial \bar{u}_{\psi}}{\partial z_{\psi}}-\frac{\partial \bar{w}_{\psi}}{\partial x_{\psi}} \\
\frac{\partial \bar{v}}{\partial x_{\psi}}-\frac{\partial \bar{u}_{\psi}}{\partial y}
\end{array}\right) .
$$

In order to obtain an equation for the wallnormal velocity component of the secondary disturbance flow field, curl is applied again to (5.16) - (5.18). The resulting equation

$$
\begin{aligned}
\left\{\frac{\partial}{\partial t}\right. & \left.+\left(U_{\psi}-C\right) \frac{\partial}{\partial x_{\psi}}+W_{\psi} \frac{\partial}{\partial z_{\psi}}-\frac{1}{R e} \Delta_{\psi}\right\} \Delta_{\psi} \bar{v}-\frac{d^{2} U_{\psi}}{d y^{2}} \frac{\partial \bar{v}}{\partial x_{\psi}}-\frac{d^{2} W_{\psi}}{d y^{2}} \frac{\partial \bar{v}}{\partial z_{\psi}} \\
& +\left\{\tilde{u}_{\psi} \frac{\partial}{\partial x_{\psi}}+\tilde{v} \frac{\partial}{\partial y}+\tilde{w}_{\psi} \frac{\partial}{\partial z_{\psi}}\right\} \Delta_{\psi} \bar{v} \\
& +\frac{\partial \tilde{u}_{\psi}}{\partial x_{\psi}}\left\{2 \frac{\partial \bar{\zeta}_{\psi}}{\partial x_{\psi}}-\Delta_{\psi} \bar{v}\right\}+\frac{\partial \tilde{v}}{\partial x_{\psi}}\left\{2 \frac{\partial \bar{\zeta}_{\psi}}{\partial y}+\Delta_{\psi} \bar{u}_{\psi}\right\}+2 \frac{\partial \tilde{w}_{\psi}}{\partial x_{\psi}} \frac{\partial \bar{\zeta}_{\psi}}{\partial z_{\psi}} \\
& -\left(\Delta_{\psi} \tilde{u}_{\psi}\right) \frac{\partial \bar{v}}{\partial x_{\psi}}+\left(\Delta_{\psi} \tilde{v}\right)\left\{\frac{\partial \bar{u}_{\psi}}{\partial x_{\psi}}-\frac{\partial \bar{w}_{\psi}}{\partial z_{\psi}}\right\}+\frac{\partial^{2} \tilde{w}_{\psi}}{\partial x_{\psi}^{2}} \frac{\partial \bar{v}}{\partial z_{\psi}}-2 \frac{\partial^{2} \tilde{w}_{\psi}}{\partial x_{\psi} \partial y} \frac{\partial \bar{u}_{\psi}}{\partial z_{\psi}}-\frac{\partial^{2} \tilde{w}_{\psi}}{\partial y^{2}} \frac{\partial \bar{v}}{\partial z_{\psi}} \\
& +\left[\frac{\partial}{\partial x_{\psi}}\left(\Delta_{\psi} \tilde{v}\right)\right] \bar{u}_{\psi}+\left[\frac{\partial}{\partial y}\left(\Delta_{\psi} \tilde{v}\right)\right] \bar{v}=0
\end{aligned}
$$

together with the wallnormal vorticity equation (5.17), the continuity equation (5.15), and the definition of vorticity (5.19) form the complete system 
of governing equations. The boundary conditions for the secondary disturbances are homogeneous at the wall and at the boundary layer edge.

Since the coefficients of equations (5.20) and (5.17) are independent of $t$ and $z_{\psi}$ and periodic in $x_{\psi}$, Floquet theory (cf. Herbert [38]) can be applied, and the solution can be expressed in normal modes of the form

$$
\left(\begin{array}{c}
\bar{u}_{\psi}\left(x_{\psi}, y, z_{\psi}, t\right) \\
\bar{v}\left(x_{\psi}, y, z_{\psi}, t\right) \\
\bar{w}_{\psi}\left(x_{\psi}, y, z_{\psi}, t\right) \\
\bar{\xi}_{\psi}\left(x_{\psi}, y, z_{\psi}, t\right) \\
\bar{\eta}\left(x_{\psi}, y, z_{\psi}, t\right) \\
\bar{\zeta}_{\psi}\left(x_{\psi}, y, z_{\psi}, t\right)
\end{array}\right)=e^{\sigma t+i\left(a_{\psi} x_{\psi}+b_{\psi} z_{\psi}\right)} \sum_{n=-N_{s}}^{N_{s}}\left(\begin{array}{c}
\bar{u}_{\psi, n}(y) \\
\bar{v}_{n}(y) \\
\bar{w}_{\psi, n}(y) \\
\bar{\xi}_{\psi, n}(y) \\
\bar{\eta}_{n}(y) \\
\bar{\zeta}_{\psi, n}(y)
\end{array}\right) e^{i n \alpha_{\psi} x_{\psi}},
$$

where the wave number $b_{\psi}$ of the secondary disturbance in direction of the primary vortex axis is real and prescribed. In the temporal approach used here, the Floquet exponent $a_{\psi}$ is real and prescribes the detuning of the primary wave number $\alpha_{\psi}$ normal to the vortex axis. The complex eigenvalue $\sigma=\sigma_{r}+i \sigma_{i}$ gives the temporal growth rate $\sigma_{r}$ and the frequency shift $\sigma_{i}$ of the secondary disturbance.

The continuity equation (5.15) and the definition of vorticity (5.19) can now be used to express $\bar{u}_{\psi, n}, \bar{w}_{\psi, n}, \bar{\xi}_{\psi, n}$ and $\bar{\zeta}_{\psi, n}$, in terms of $\bar{v}_{n}$ and $\bar{\eta}_{n}$ :

$$
\begin{aligned}
& \bar{u}_{\psi, n}=\frac{i(\delta+n) \alpha_{\psi}}{k_{n}^{2}} \frac{d \bar{v}_{n}}{d y}-\frac{i b_{\psi}}{k_{n}^{2}} \bar{\eta}_{n} \\
& \bar{w}_{\psi, n}=\frac{i b_{\psi}}{k_{n}^{2}} \frac{d \bar{v}_{n}}{d y}+\frac{i(\delta+n) \alpha_{\psi}}{k_{n}^{2}} \bar{\eta}_{n} \\
& \bar{\xi}_{\psi, n}=-i b_{\psi} \bar{v}_{n}+\frac{i b_{\psi}}{k_{n}^{2}} \frac{d^{2} \bar{v}_{n}}{d y^{2}}+\frac{i(\delta+n) \alpha_{\psi}}{k_{n}^{2}} \frac{d \bar{\eta}_{n}}{d y} \\
& \bar{\zeta}_{\psi, n}=i(\delta+n) \alpha_{\psi} \bar{v}_{n}-\frac{i(\delta+n) \alpha_{\psi}}{k_{n}^{2}} \frac{d^{2} \bar{v}_{n}}{d y^{2}}+\frac{i b_{\psi}}{k_{n}^{2}} \frac{d \bar{\eta}_{n}}{d y}
\end{aligned}
$$

where

$$
\delta=\frac{a_{\psi}}{\alpha_{\psi}}
$$

and

$$
k_{n}^{2}=(\delta+n)^{2} \alpha_{\psi}^{2}+b_{\psi}^{2} .
$$

Thus, introducing (5.10) and (5.21) into equations (5.20) and (5.17) yields a system of ordinary differential equations for the secondary disturbance modes $\left(\bar{v}_{n}, \bar{\eta}_{n}\right)$ :

$$
\left\{\left[\sigma+i(n+\delta) \alpha_{\psi}\left(U_{\psi}-C\right)+i b_{\psi} W_{\psi}\right]\left(\frac{d^{2}}{d y^{2}}-\left[(n+\delta)^{2} \alpha_{\psi}^{2}+b_{\psi}^{2}\right]\right)\right.
$$




$$
\begin{gathered}
\left.-\frac{1}{R e}\left(\frac{d^{2}}{d y^{2}}-\left[(n+\delta)^{2} \alpha_{\psi}^{2}+b_{\psi}^{2}\right]\right)^{2}-i(n+\delta) \alpha_{\psi} \frac{d^{2} U_{\psi}}{d y^{2}}-i b_{\psi} \frac{d^{2} W_{\psi}}{d y^{2}}\right\} \bar{v}_{n} \\
+\sum_{m=-N_{p}}^{N_{p}} S_{n-m, m}^{O S}=0 \\
\left\{\sigma+i(n+\delta) \alpha_{\psi}\left(U_{\psi}-C\right)+i b_{\psi} W_{\psi}-\frac{1}{R e}\left(\frac{d^{2}}{d y^{2}}-\left[(n+\delta)^{2} \alpha_{\psi}^{2}+b_{\psi}^{2}\right]\right)\right\} \bar{\eta}_{n} \\
+\left\{i b_{\psi} \frac{d U_{\psi}}{d y}-i(n+\delta) \alpha_{\psi} \frac{d W_{\psi}}{d y}\right\} \bar{v}_{n}+\sum_{m=-N_{p}}^{N_{p}} S_{n-m, m}^{S Q}=0
\end{gathered}
$$

The complete structure of the convolution terms $S_{n-m, m}^{O S}$ and $S_{n-m, m}^{S Q}$, which are functions of the primary disturbance velocity profiles, truncated at $m= \pm N_{p}$, is given in Koch et al. [53].

\subsection{Numerical Solution Procedure}

Equations (5.28) and (5.29) constitute an eigenvalue problem for the temporal eigenvalue $\sigma$. For numerical solution they are - with consideration of the corresponding homogeneous boundary conditions - transformed into algebraic form by means of Chebyshev collocation. In order to sufficiently resolve the region near the edge of the boundary layer, where the maxima of the most amplified secondary disturbance eigenfunctions are expected, the physical domain $y \in\left[0, y_{e}\right]$ in the direction normal to the plate is mapped onto the computational domain $\chi \in(0,1]$ through a convolution of two mappings (cf. Erlebacher \& Hussaini [21]). A hyperbolic tangent mapping takes $\chi$ to an intermediate variable $\psi$ according to

$$
\frac{\chi-\chi_{\text {crit }}}{\Delta \chi}=t_{\epsilon} \tanh \left(\frac{\psi-\psi_{\text {crit }}}{\Delta \psi}\right),
$$

before the exponential mapping

$$
\psi=e^{-\frac{y}{y_{0}}}, \quad y=-y_{0} \ln \psi
$$

stretches the maps $\psi$ onto the physical variable $y$. In the computational domain, the $K$ collocation points are distributed as follows:

$$
\chi_{j}=\cos \left(\frac{j \pi}{2 K-1}\right), \quad j=0, \ldots, K-1 .
$$

While mapping (5.31) increases the density of collocation points in the region near the wall, mapping (5.30) concentrates nodes about

$$
\psi_{\text {crit }}=e^{-\frac{y_{\text {crit }}}{y_{0}}},
$$


where $y_{\text {crit }}$ is a physical location near the edge of the boundary layer, e.g. $y_{\text {crit }}=\delta_{99.9}$.

$$
\Delta \psi=-\frac{\Delta y \cdot \psi_{c r i t}}{y_{0}}
$$

measures the width of the concentration region, and $t_{\epsilon}$ controls the degree of influence of the hyperbolic tangent term in (5.30). The quantities $\chi_{\text {crit }}$ and $\Delta \chi$ in mapping (5.30) are determined by

$$
\lim _{\psi \rightarrow 0} \chi(\psi)=0 \quad \text { and }\left.\quad \chi(\psi)\right|_{\psi=1}=1 .
$$

For the computations presented in this chapter, $K=55$ collocation points are used. Once a fairly good estimate of the eigenvalue spectrum is found by using an efficient global solver, particular eigenvalues of interest - these are mainly the unstable ones - are refined using local Wielandt iteration (cf. Zurmühl [98]).

\subsection{High Frequency Secondary Instability}

In this section, a base flow distorted by stationary crossflow vortices is investigated with respect to temporal secondary instability. As primary disturbance, a crossflow vortex with spanwise wave number $\beta_{c}=261.8$ and initial amplitude $\left|\tilde{u}_{c}\right|_{\max }=7.2 \cdot 10^{-5}$ at $x_{c}=0.06$ is considered. The flow field distorted by this vortex and its 16 harmonics is described in detail in section 4.3. For these initial conditions of the primary disturbance, secondary instability can be investigated for a large range of primary vortex amplitudes. As can be seen from figure 4.11, strong nonlinear behavior can be observed downstream of $x_{c}=0.7$, but the vortex amplitude continues to grow monotonically up to the end of the plate. The amplitudes of the fundamental vortex $(0,-1,1)$, its first harmonic $(0,-2,2)$, and the mean flow distortion $(0,0,0)$ are summarized in table 5.1 for the chordwise locations discussed in this section. In order to make comparisons with results of other studies, which use local reference quantities, easy, the quantity $Q_{e}^{*}\left(x_{c}\right) / Q_{\infty}^{*}$ is also included in table 5.1 .

Unless otherwise mentioned, the detuning parameter is $\delta=0$, i.e. only the fundamental type of secondary disturbances is considered. This restriction is supported by the results of Fischer et al. [24], who found that the choice of the detuning parameter only insignificantly affects the amplification rates of high-frequency secondary disturbances in a Falkner-Skan-Cooke flow, and of Balachandar et al. [5], who made similar observations for a rotating-disk flow. 


\begin{tabular}{||c|c|c|c|c||}
\hline \hline \multirow{2}{*}{$x_{c}$} & \multicolumn{3}{|c|}{$\left|\tilde{u}_{c \mid}\right|_{\max } \times 100$} & \multirow{2}{*}{$Q_{e}^{*} / Q_{\infty}^{*}$} \\
\cline { 2 - 4 } & $(0,-1,1)$ & $(0,0,0)$ & $(0,-2,2)$ & \\
\hline \hline 0.40 & 0.34 & 0.0028 & 0.0028 & 0.8205 \\
\hline 0.60 & 3.9 & 0.32 & 0.32 & 0.8743 \\
\hline 0.65 & 6.7 & 0.95 & 0.93 & 0.8876 \\
\hline 0.70 & 10.6 & 2.3 & 2.3 & 0.9007 \\
\hline 0.75 & 14.2 & 4.0 & 4.3 & 0.9122 \\
\hline 0.80 & 17.3 & 5.3 & 7.0 & 0.9250 \\
\hline 0.95 & 23.1 & 14.2 & 12.1 & 0.9623 \\
\hline \hline
\end{tabular}

Table 5.1: Amplitude of primary disturbance modes and $Q_{e}^{*} / Q_{\infty}^{*}$ at different positions on the plate.

\subsubsection{Limit of Low Primary Vortex Amplitude}

At very low amplitudes of the primary vortex, no unstable high-frequency mode can be expected. As can be seen from figure 5.1, the global spectrum at $x_{c}=0.4$ contains two unstable eigenvalues. These, however, refer to the same physical disturbance mode: The spectra for negative values of $b_{\psi}$ are complex conjugates to the spectra for the corresponding positive values of $b_{\psi}$, i.e. the sign of the frequency of the disturbance mode flips with the sign of $b_{\psi}$.

The dependence of the temporal growth rate $\sigma_{r}$ on the wave number $b_{\psi}$ is shown in figure 5.2, which is symmetric around $b_{\psi}=0$. The maximal temporal growth rate of the disturbance mode is $\sigma_{r}=3.85$ for $b_{\psi}=26.5$, where $\sigma_{i}=13.5\left(\hat{=} f^{*}=81.7 \mathrm{~Hz}\right)$. This mode corresponds to a primary traveling crossflow wave, as can be seen from figure 5.3, where the $\left|\bar{w}_{\psi}\right|$-profile of the disturbance obtained by secondary analysis is compared to the $\left|\bar{w}_{\psi}\right|$-profile of a disturbance with the same wave number obtained using parallel primary instability theory. The good agreement between the two velocity profiles also serves as a check of the tools used for secondary instability analysis in the limit of low primary disturbance amplitude.

A $\left|\bar{w}_{\psi}\right|$-isocontour plot of this low-frequency "secondary" disturbance is shown in figure 5.4 (top). The dashed lines indicate the $W_{\psi}$-component of the total (primarily disturbed) flow field and show the very small influence of the primary crossflow vortex on the laminar base flow at $x_{c}=0.4$. The contour spacing used for plotting the primarily distorted base flow is $0.05 Q_{\infty}^{*}$ in all figures of the type of figure 5.4, unless otherwise mentioned. Note, that 

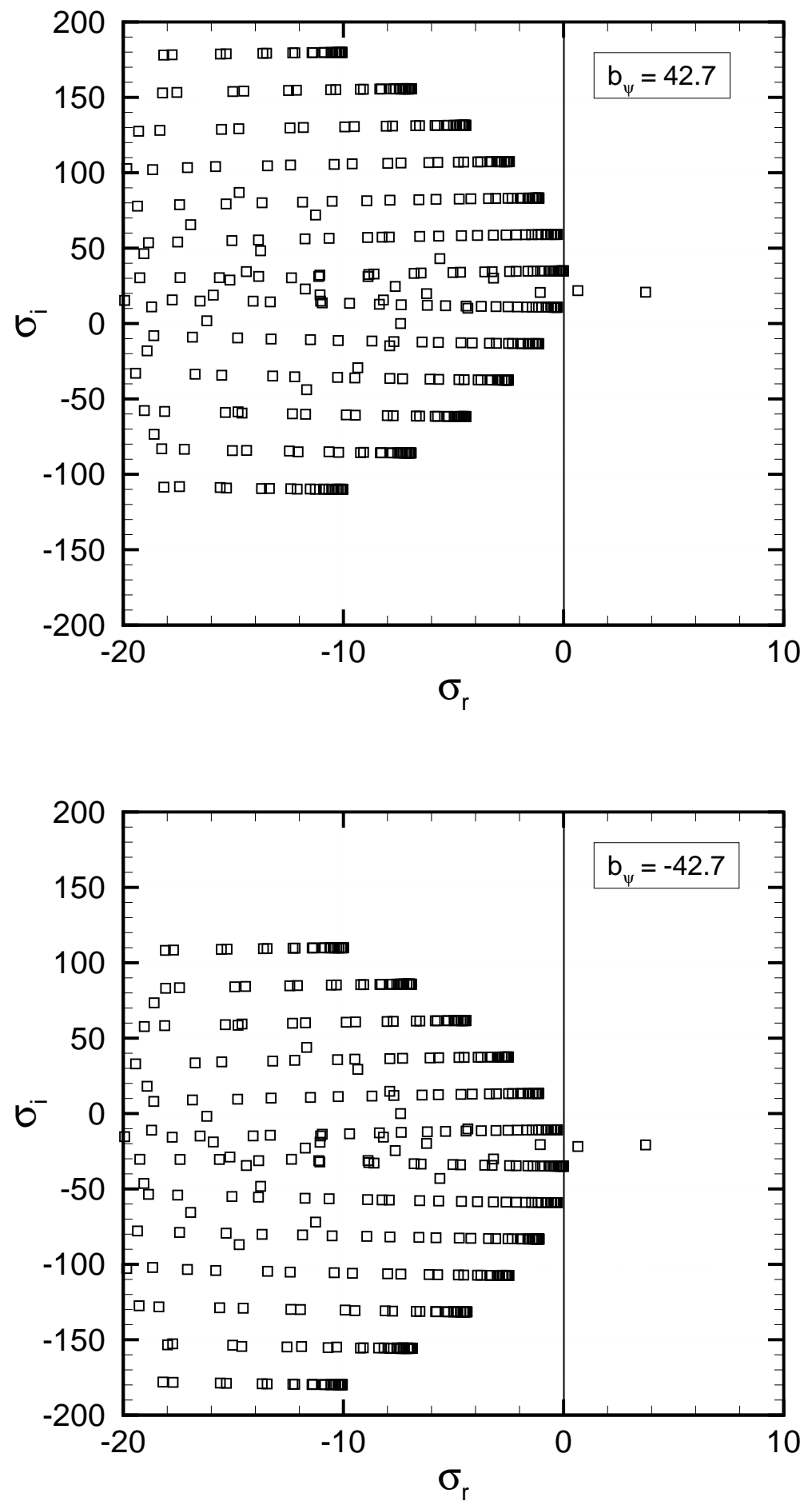

Figure 5.1: Temporal eigenvalue spectra at $x_{c}=0.4$ with $N_{s}=6$ for $b_{\psi}=42.7$ (top) and $b_{\psi}=-42.7$ (bottom). 


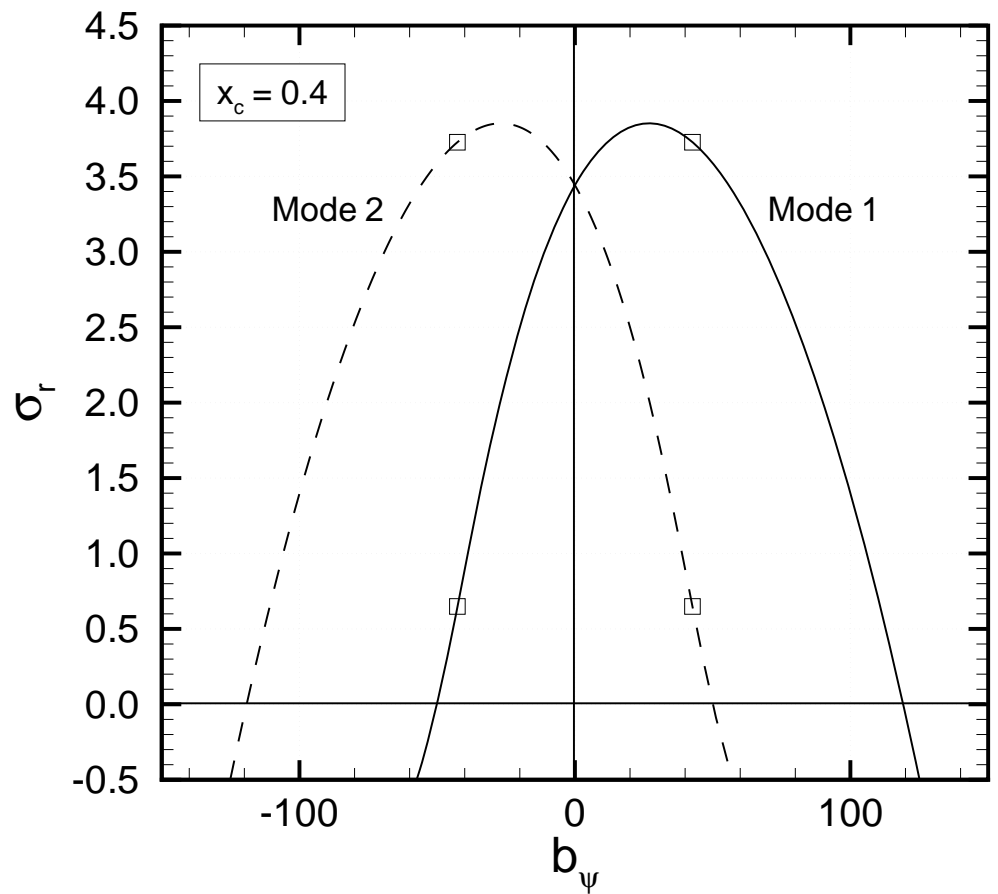

Figure 5.2: Temporal growth rate $\sigma_{r}$ of secondary disturbance modes versus wave number $b_{\psi}$ at $x_{c}=0.4\left(N_{s}=6\right)$. The square symbols denote the unstable eigenvalues shown in figure 5.1

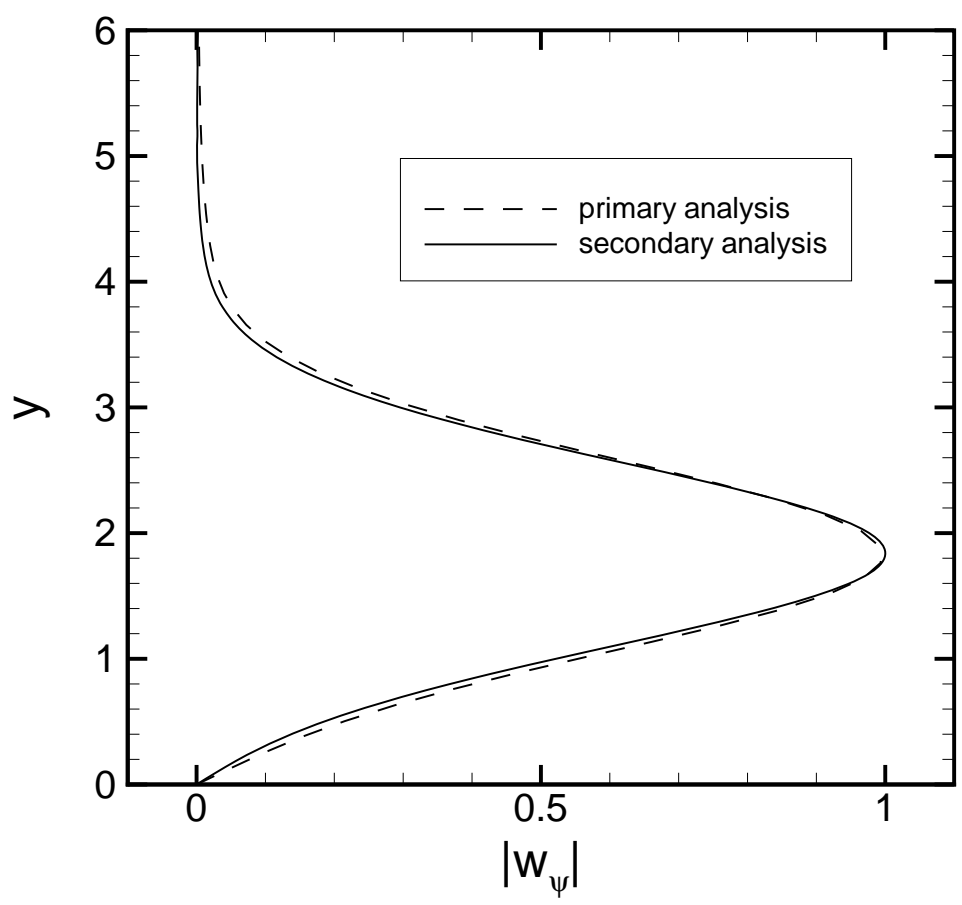

Figure 5.3: Comparison of $\left|\bar{w}_{\psi}\right|$-velocity profiles computed using primary (parallel) and secondary instability theory in the limit of low primary vortex amplitude. 

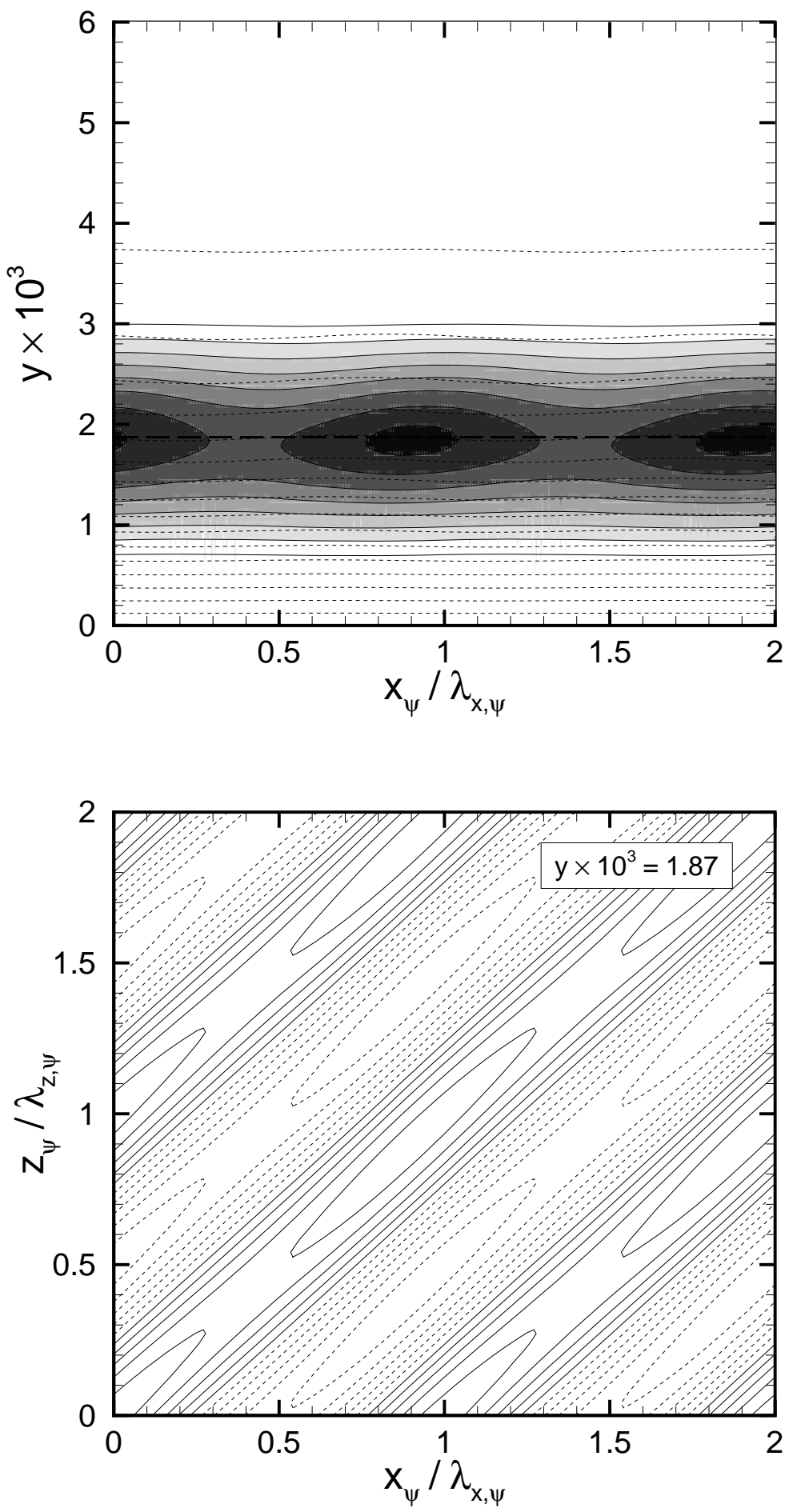

Figure 5.4: Top: $\left|\bar{w}_{\psi}\right|$-isolines of secondary disturbance mode with $b_{\psi}=26.5$ at $x_{c}=0.4\left(N_{s}=6\right)$. The dashed lines indicate the $W_{\psi}$-component of the total (primarily disturbed) flow field. Bottom: $\bar{w}_{\psi}$-isolines in a wallparallel plane at distance $y=1.87 \cdot 10^{-3}$ from the wall. At this location (indicated by the long-dashed line in the top figure), the maximum of $\left|\bar{w}_{\psi}\right|$ is found. 
the upper figure depicts the modulus of $\bar{w}_{\psi}$ (corresponding to the rms-value of the secondary disturbance). A single disturbance wave would, hence, appear as a set of wallparallel iso-lines. The elliptical structures in figure 5.4 (top) correspond to small modulations of the disturbance wave by the lowamplitude stationary crossflow vortex. This influence is also illustrated by figure 5.4 (bottom), which shows $\bar{w}_{\psi}$-isolines in that wallparallel plane (marked by the long-dashed line in the upper figure), where the maximum of $\left|\bar{w}_{\psi}\right|$ is found. As expected, further unstable eigenvalues could not be found by a variation of $b_{\psi}$ at this chordwise position.

\subsubsection{Propagation of Secondary Disturbances}

Before structure and growth of secondary disturbances are discussed in the following subsections, the propagation of these disturbances is briefly considered here. The solutions obtained by using primary linear instability theory (see chapter 3 ) represent waves and vortices, which - with respect to a given coordinate system and at some given $x_{c}$-position - each have uniquely defined wave numbers $\alpha$ and $\beta$. The local direction of wave propagation is then normal to the lines of constant phase (cf. equation (3.31)), the phase speed is

$$
c=\frac{\omega}{\sqrt{\alpha^{2}+\beta^{2}}} .
$$

In the Floquet formulation (5.21), each normal mode is associated with its own wave number in $x_{\psi}$-direction (i.e. $a_{\psi}+n \alpha_{\psi}$ ), while the variation in $z_{\psi}$ direction is captured by the single wave number $b_{\psi}$. As a consequence, in the frame of Floquet theory, the complete structure of the secondary disturbance can only propagate in $z_{\psi}$-direction with propagation velocity

$$
\bar{c}=\frac{-\sigma_{i}}{b_{\psi}} .
$$

Only in the limit of zero primary vortex amplitude, equations (5.28) and (5.29) decouple for the different normal modes. The disturbance corresponding to a primary crossflow wave (see subsection 5.4 .1 ) is captured by a single normal mode. In this case, it is possible and appropriate to compute a phase velocity normal to the constant phase lines.

The propagation velocity of secondary disturbances in the direction of the primary vortex axis has been investigated both experimentally and numerically in previous studies. In a secondary instability analysis of Görtler vortices, Li \& Malik [56] found the propagation velocity of secondary modes to be about $70 \%$ of the velocity at the boundary-layer edge and showed that the amplitudes of the secondary modes are concentrated in the region corresponding to the critical layer in the case of neutral stability. Similar results have been obtained by Bottaro \& Klingmann [13]. Wang et al. [95] 
analyzed the secondary instability of swept Hiemenz flow and obtained significantly higher propagation speeds especially for the modes, which exhibit very high growth rates at high primary vortex amplitudes. This observation is confirmed by the results of the present study.

\subsubsection{Onset of High Frequency Secondary Instability}

The first additional unstable modes appear in the spectrum at $x_{c}=0.6$, where the amplitude $\left|\tilde{u}_{c}\right|_{\max }$ of the primary vortex is about $4 \%$ of the free stream velocity. Figures 5.5 and 5.6 show spectra for different wave numbers $b_{\psi}$ at this location. For $b_{\psi}=76.2$ (figure 5.5), three unstable eigenvalues exist. Two of them belong to the disturbance mode related to the primary crossflow wave, as discussed in subsection 5.4.1. The growth rate of this wave clearly exceeds that of the other modes found at this chordwise location. While, for $b_{\psi}=152.5$, the global spectrum does not contain any unstable eigenvalue, a slightly unstable mode appears again for $b_{\psi}=228.7$ (see figure 5.8). For $b_{\psi}$ significantly greater than 260 , no unstable eigenvalues could be found at $x_{c}=0.6$.

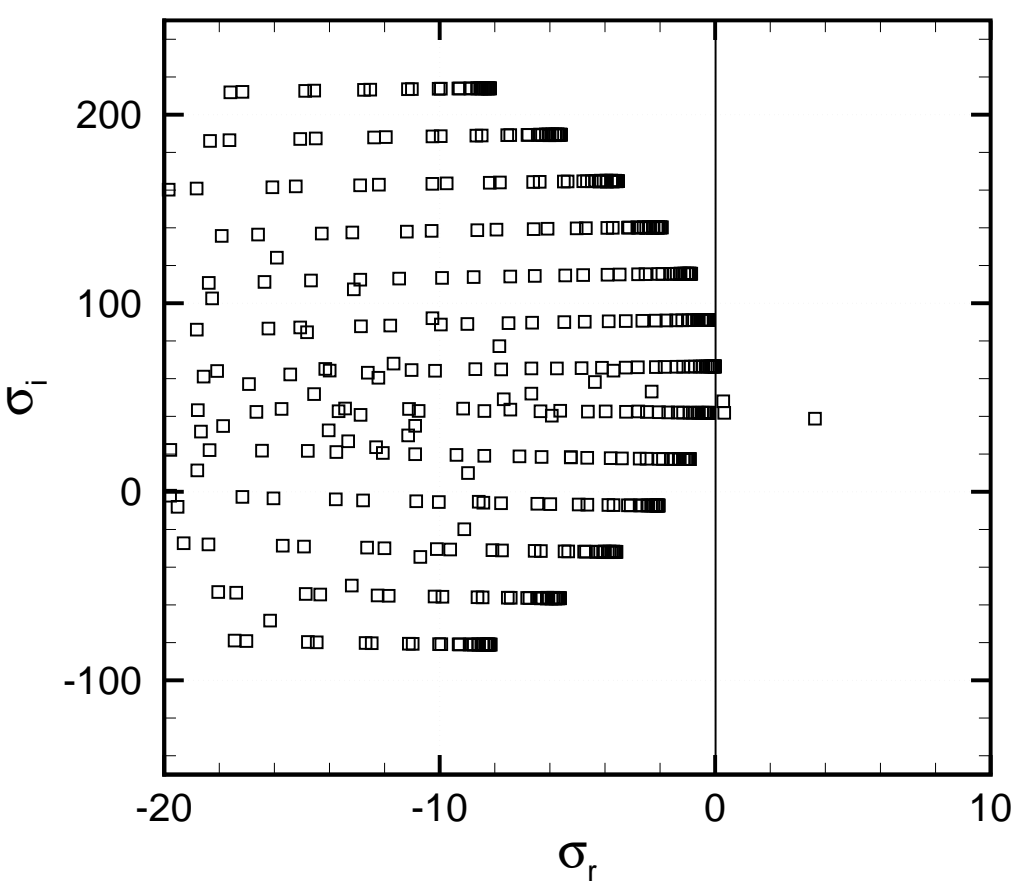

Figure 5.5: Temporal eigenvalue spectrum at $x_{c}=0.6$ for $b_{\psi}=76.2\left(N_{s}=6\right)$.

A study of the dependence of the global spectra on the number of normal modes considered in the secondary instability analysis is shown in figure 5.6. For each normal mode - the number of these is $2 N_{s}+1$ (see eqn. (5.21)) - one branch in the continuous part of the spectrum appears (cf. Hein et al. [34]). Of particular interest is the dependence of the unstable eigenvalue 

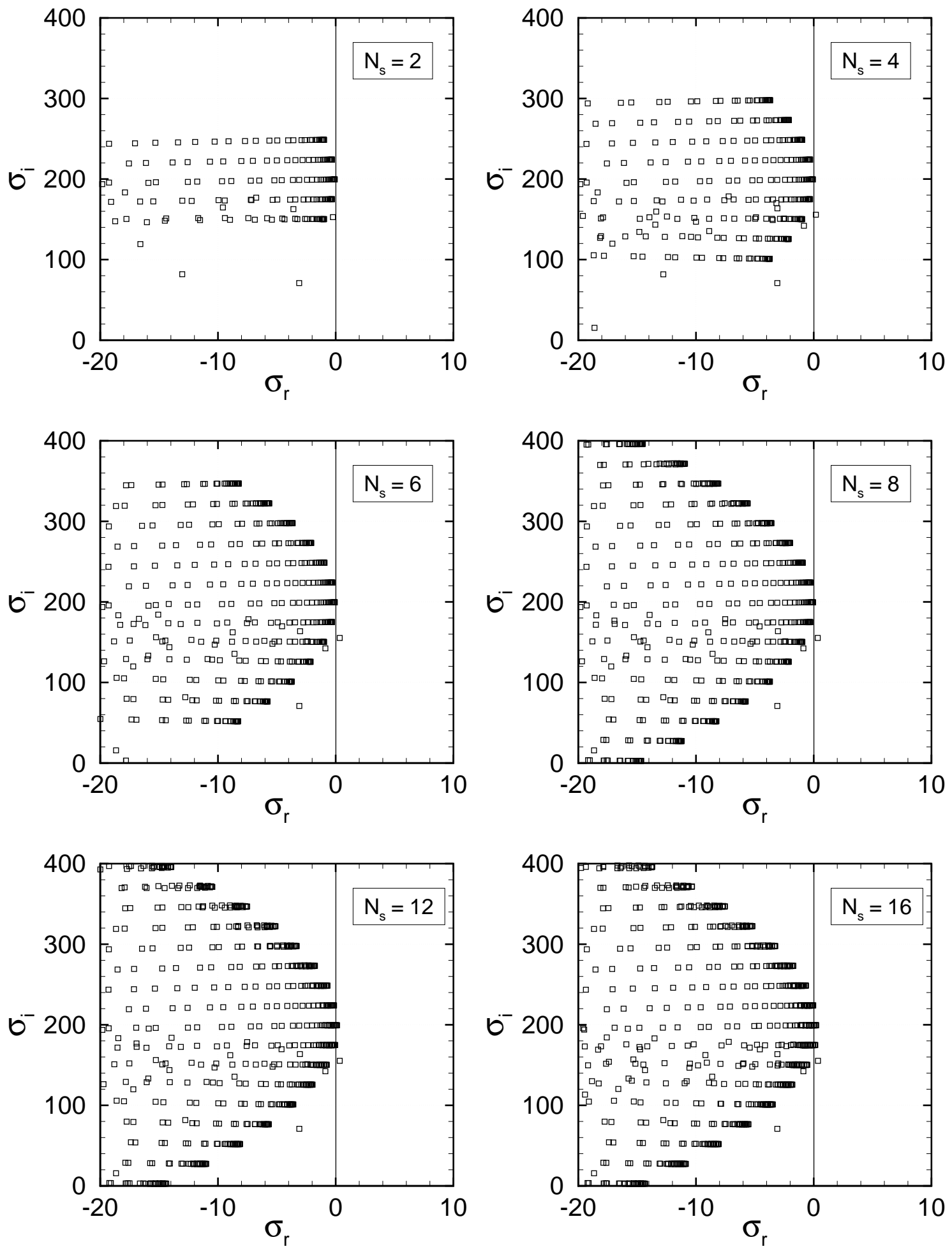

Figure 5.6: Temporal secondary eigenvalue spectra for $b_{\psi}=228.7$ at $x_{c}=0.6$ with $N_{p}=16$ and different $N_{S}$. 
on $N_{s}$. For $N_{s}=2$ (i.e. 5 normal modes) this eigenvalue is not even unstable; for $N_{s}=4$ the growth rate $\sigma_{r}$ of this mode is about $50 \%$ of that obtained for $N_{s}=6$. Increasing the number of normal modes further does not significantly change this eigenvalue. However, if $N_{s}$ is increased to 16 , the growth rate $\sigma_{r}$ of the disturbance deviates again from the value obtained for $N_{s}=6 \ldots 12$. This is probably due to problems with the numerical condition of the system. The results of this resolution study are summarized in figure 5.7 .

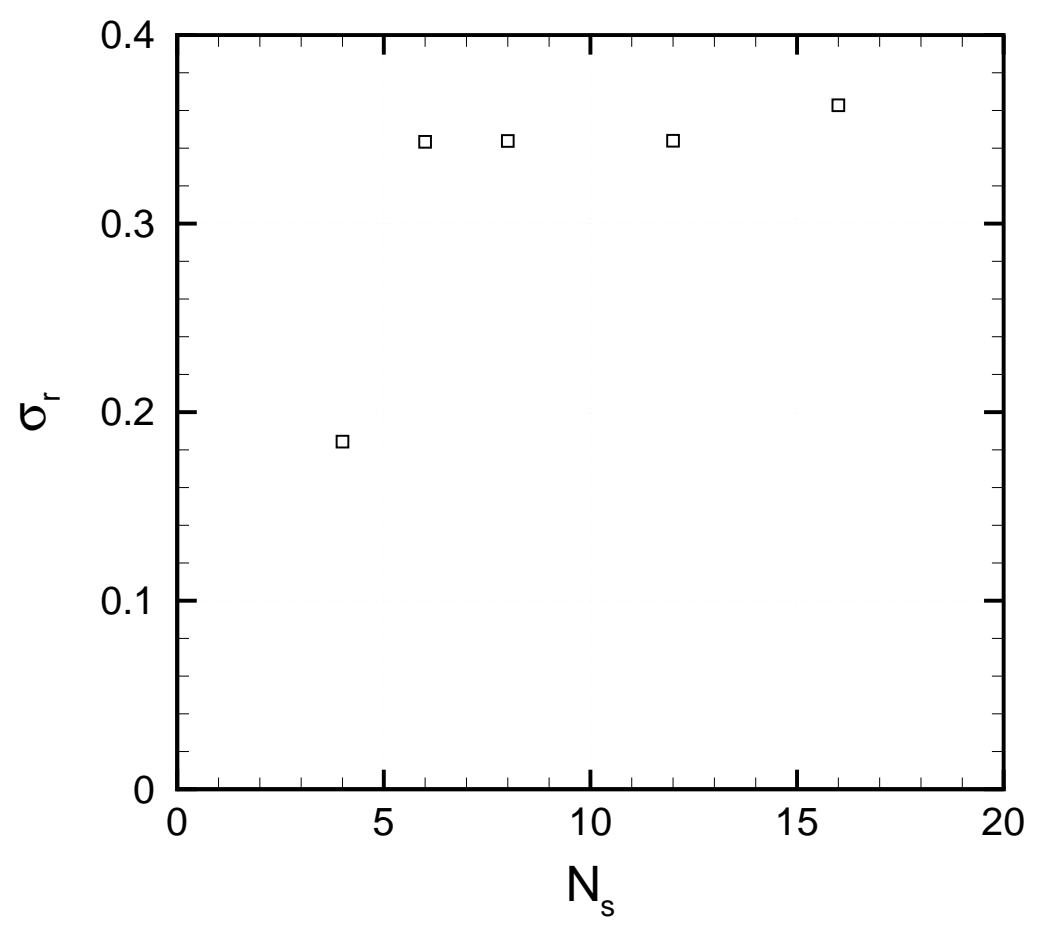

Figure 5.7: Temporal growth rate $\sigma_{r}$ of secondary disturbance with $b_{\psi}=228.7$ at $x_{c}=0.6$ for varying number of normal modes.

Figure 5.8 summarizes the growth rates of the disturbances for varying $b_{\psi}$. Some properties of these modes for the wave numbers $b_{\psi}$, where they exhibit the highest growth rates $\sigma_{r}$, are listed in table 5.2.

\begin{tabular}{||c|c|c|c|c|c||}
\hline \hline Mode & $b_{\psi}$ (maximal growth) & $\sigma_{r}$ & $\sigma_{i}$ & $f^{*}[\mathrm{~Hz}]$ & $\sigma_{i} / b_{\psi}$ \\
\hline \hline 1 & 41.2 & 4.621 & 21.6 & 131 & 0.524 \\
\hline 2 & 73.2 & 0.351 & 39.8 & 241 & 0.544 \\
\hline 3 & 236.3 & 0.416 & 160.8 & 975 & 0.680 \\
\hline \hline
\end{tabular}

Table 5.2: Properties of unstable secondary disturbance modes at $x_{c}=0.6$. 


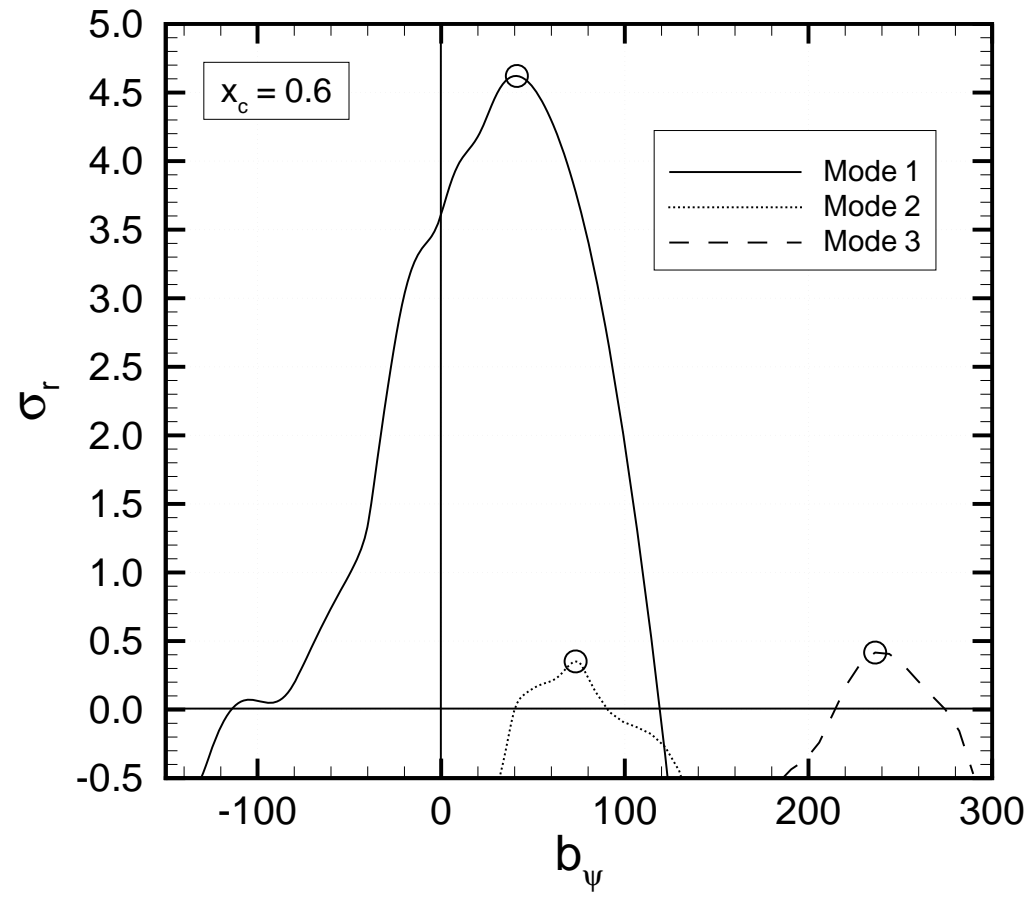

Figure 5.8: Temporal growth rate $\sigma_{r}$ of secondary disturbance modes versus wave number $b_{\psi}$ at $x_{c}=0.6\left(N_{s}=6\right)$. The circles indicate the locations, where the $\left|\bar{w}_{\psi}\right|$-isolines shown in figures $5.9-5.11$ have been extracted.

For mode $1,\left|\bar{w}_{\psi}\right|$-isolines in the $x_{\psi}-y$-plane and $\bar{w}_{\psi}$-isolines in a wallparallel plane near the maximum of $\left|\bar{w}_{\psi}\right|$ are shown in figure 5.9. In comparison with the case of low primary vortex amplitude (see figure 5.4), the strong modulation of the secondary disturbance by the primary crossflow vortex can be seen. $\left|\bar{w}_{\psi}\right|$-isolines of mode 2 and mode 3 are shown in figures 5.10 and 5.11 , respectively. 

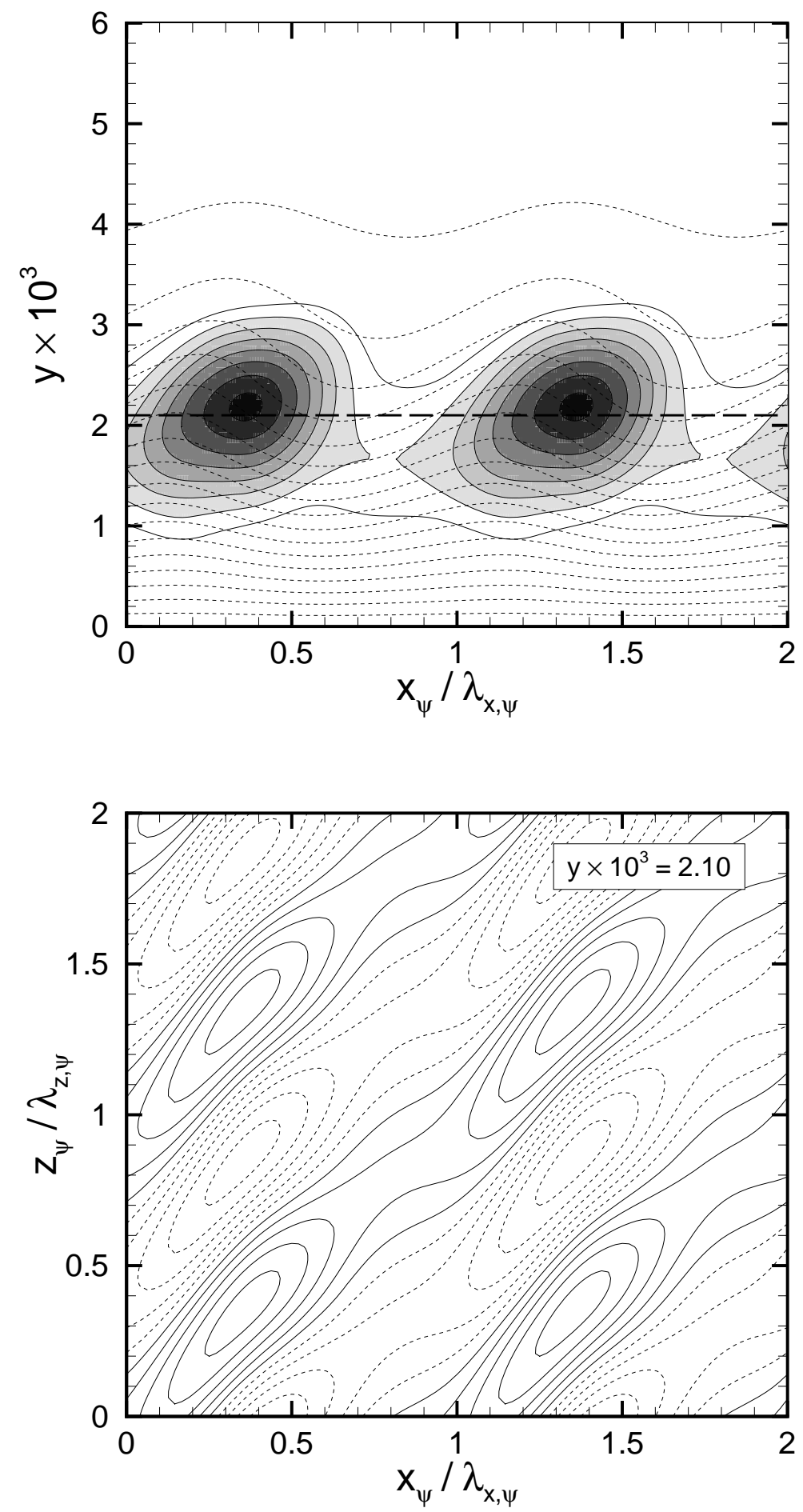

Figure 5.9: Top: $\left|\bar{w}_{\psi}\right|$-isolines of secondary disturbance (mode 1) with $b_{\psi}=$ 41.2 at $x_{c}=0.6\left(N_{s}=6\right)$. The dashed lines indicate the $W_{\psi}$-component of the total (primarily disturbed) flow field. Bottom: $\bar{w}_{\psi}$-isolines in a wallparallel plane at distance $y=2.10 \cdot 10^{-3}$ from the wall, as indicated by the longdashed line in the top figure. 


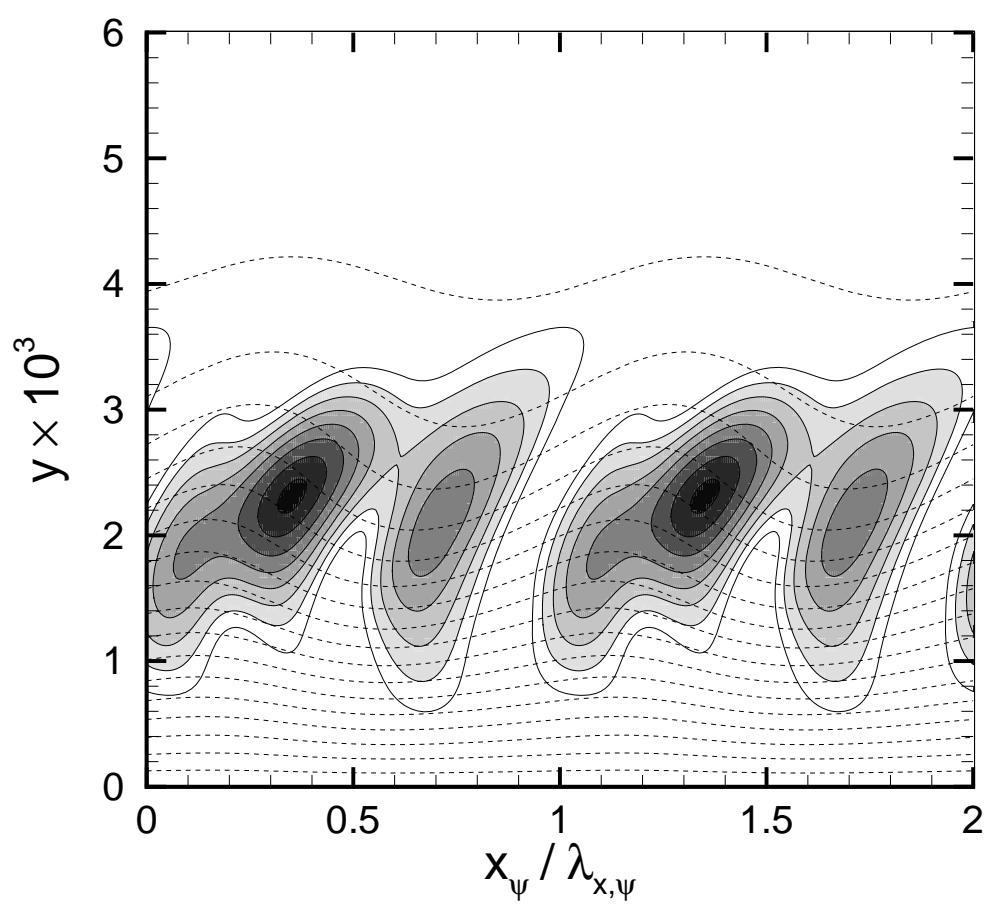

Figure 5.10: $\left|\bar{w}_{\psi}\right|$-isolines of secondary disturbance (mode 2 ) with $b_{\psi}=73.2$ at $x_{c}=0.6\left(N_{s}=6\right)$. The dashed lines indicate the $W_{\psi}$-component of the total (primarily disturbed) flow field.

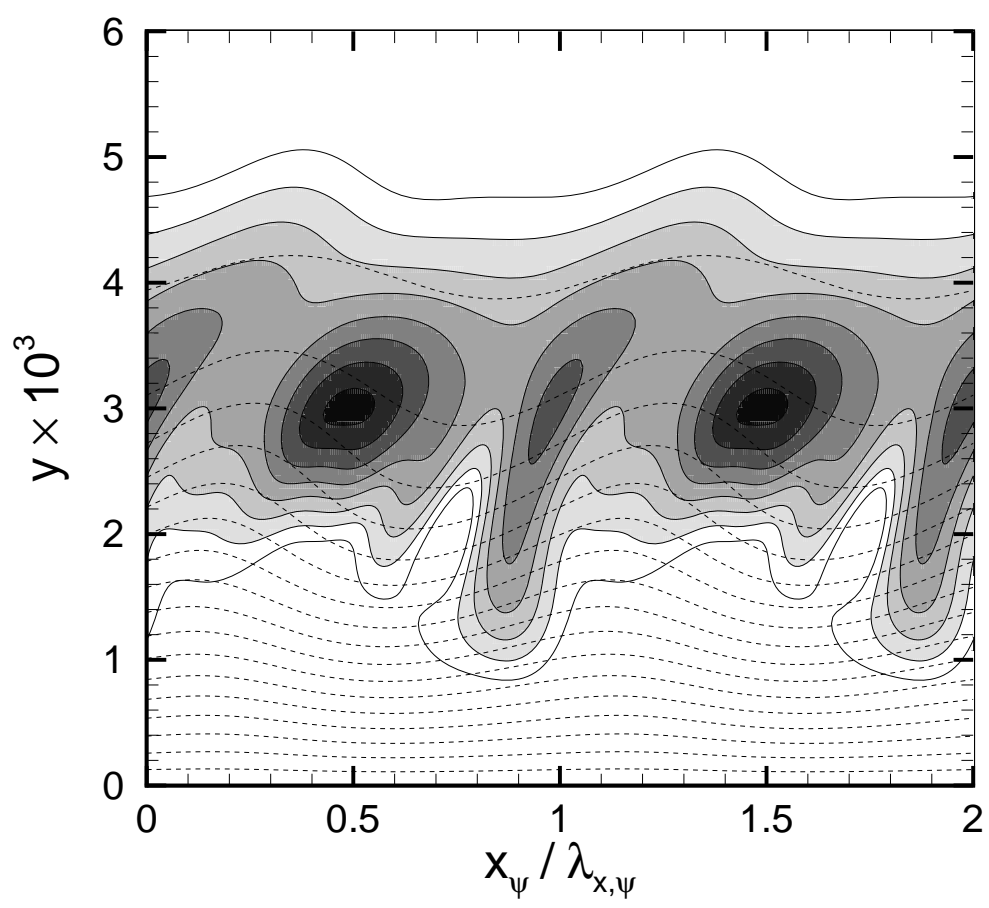

Figure 5.11: $\left|\bar{w}_{\psi}\right|$-isolines of secondary disturbance (mode 3 ) with $b_{\psi}=236.3$ at $x_{c}=0.6\left(N_{s}=6\right)$. The dashed lines indicate the $W_{\psi}$-component of the total (primarily disturbed) flow field. 
At $x_{c}=0.65$, the variety of unstable modes is still rather easy to survey. Figure 5.12 summarizes the growth rates of the disturbances as function of the wave number $b_{\psi}$ in the direction of the primary vortex axis. The highest temporal growth rate $\sigma_{r}$ is found for a disturbance with $b_{\psi}=52.2$. This mode has a frequency $f^{*}=174 \mathrm{~Hz}$, i.e. it can not be considered a high-frequency secondary instability. $\left|\bar{w}_{\psi}\right|$-isolines of this disturbance are shown in figure 5.13 . They look similar to those obtained for mode 1 at $x_{c}=0.6$. The properties of unstable modes are listed in table 5.3 for those wave numbers $b_{\psi}$, where the disturbances exhibit the highest growth rates $\sigma_{r} .\left|\bar{w}_{\psi}\right|$-isolines of some modes, indicated by circles in figure 5.12, are shown in figures 5.13-5.15.

\begin{tabular}{||c|c|c|c|c|c||}
\hline \hline Mode & $b_{\psi}$ (maximal growth) & $\sigma_{r}$ & $\sigma_{i}$ & $f^{*}[H z]$ & $\sigma_{i} / b_{\psi}$ \\
\hline \hline 1 & 52.2 & 5.40 & 28.8 & 174 & 0.552 \\
\hline 2 & 108.1 & 1.73 & 74.1 & 448 & 0.685 \\
\hline 3 & 193.8 & 1.55 & 138.1 & 835 & 0.713 \\
\hline 4 & 201.3 & 0.18 & 134.4 & 813 & 0.668 \\
\hline \hline
\end{tabular}

Table 5.3: Properties of unstable secondary disturbance modes at $x_{c}=0.65$.

A significantly greater variety of unstable secondary disturbances is found at $x_{c}=0.7$. Even though the fundamental primary crossflow vortex has an $\left|\tilde{u}_{c}\right|_{\max }$-amplitude of only $10.6 \%$ at this location, a number of secondary modes with growth rates comparable to that of the primary disturbance can be found. The temporal growth rates $\sigma_{r}$ of these modes are summarized in figure 5.16. Figure 5.17 shows their angular frequencies $\sigma_{i}$, which for each mode is approximately proportional to the wave number $b_{\psi}$ in direction of the primary vortex axis. Furthermore, for some given $b_{\psi}$ the frequencies of all unstable secondary disturbances lie within a rather narrow band.

Figures $5.18-5.22$ show $\left|\bar{w}_{\psi}\right|$-isocontours of some selected secondary disturbances as indicated by circles in figure 5.16. Main properties of these modes are summarized in table 5.4.

The highest temporal growth rate reached at $x_{c}=0.7$ is $\sigma_{r}=5.75$. The wave number $b_{\psi}$ of the corresponding disturbance is $343.2 .\left|\bar{w}_{\psi}\right|$-isocontours of this mode are shown in figure 5.18. Even though the distortion of the flow field by the primary vortex is still rather moderate, a clear correlation between the region of maximal secondary disturbance amplitude $\left|\bar{w}_{\psi}\right|$ and spanwise shear of the flow field $W_{\psi}$ can be observed. Following the nomenclature used by Malik et al. [62] and Koch et al. [53], this mode is therefore classified a "mode I"-type disturbance. Note, that roman numbering refers to a classification of secondary disturbances, while arabic numbering is used to label the modes (in a rather arbitrary way). 


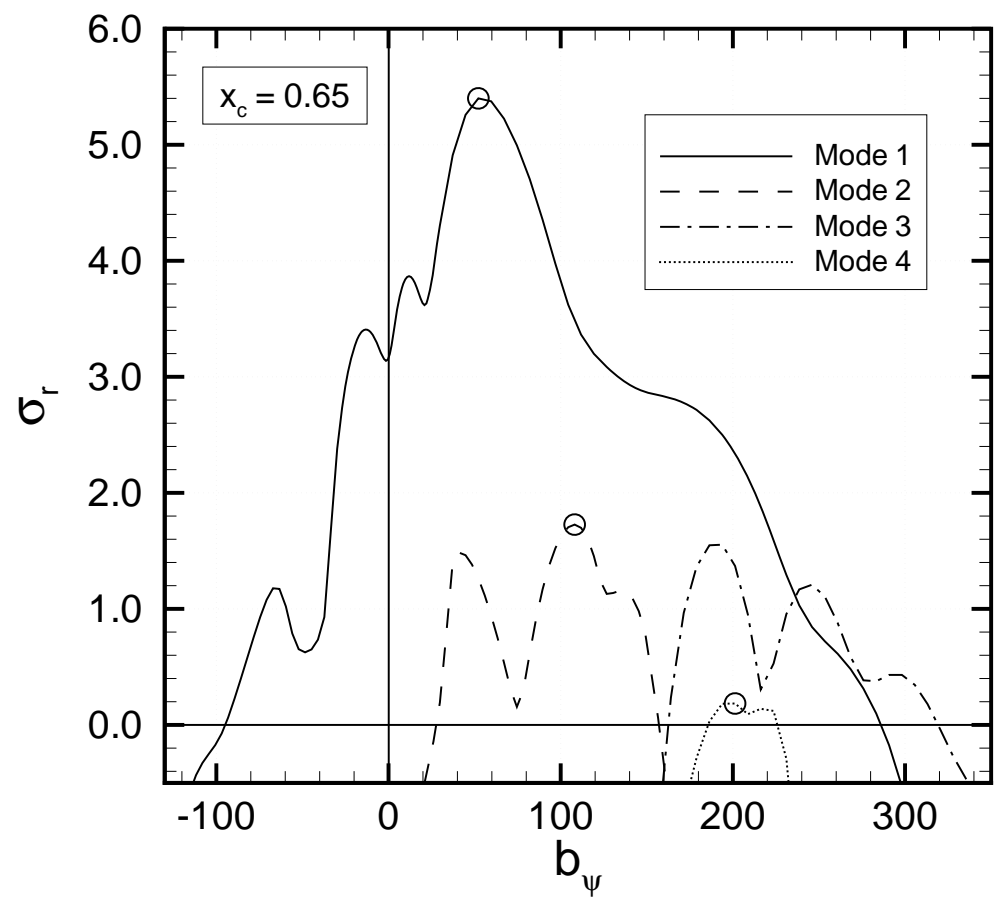

Figure 5.12: Temporal growth rate $\sigma_{r}$ of secondary disturbance modes versus wave number $b_{\psi}$ at $x_{c}=0.65\left(N_{s}=12\right)$. The circles indicate the locations, where the $\left|\bar{w}_{\psi}\right|$-isolines shown in figures $5.13-5.15$ have been extracted.

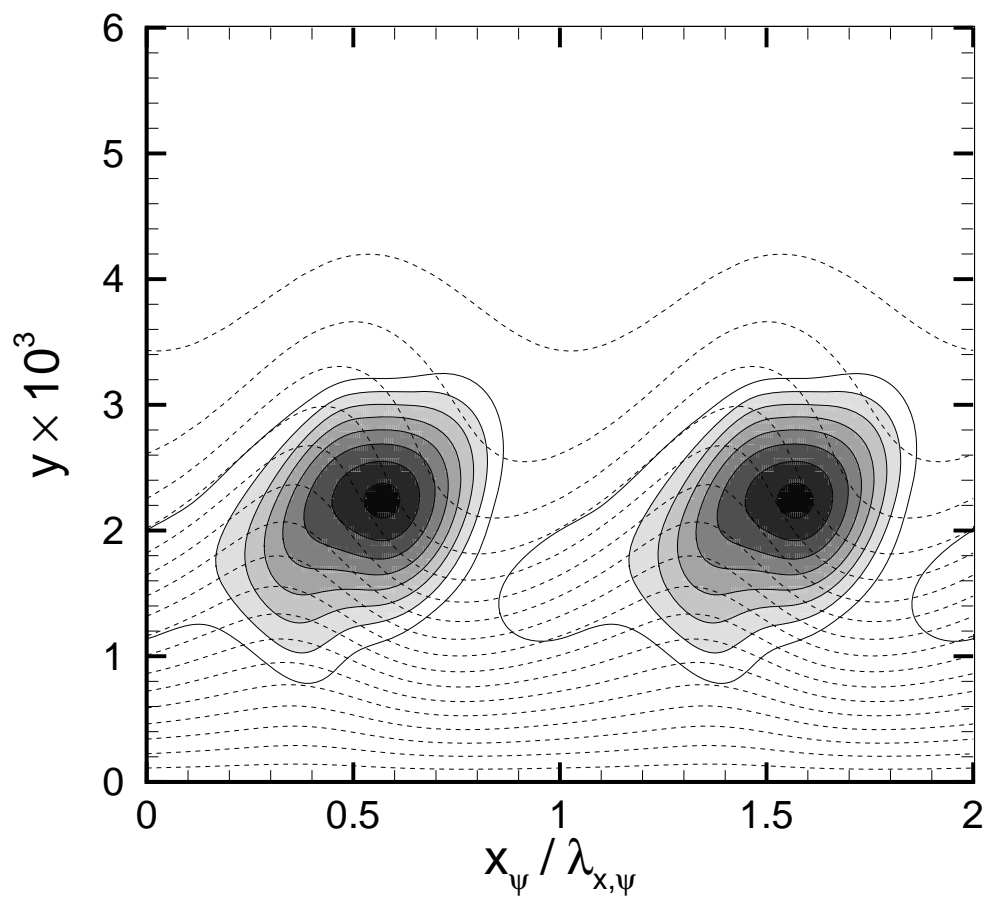

Figure 5.13: $\left|\bar{w}_{\psi}\right|$-isolines of secondary disturbance (mode 1 ) with $b_{\psi}=52.2$ at $x_{c}=0.65\left(N_{s}=12\right)$. The dashed lines indicate the $W_{\psi}$-component of the total (primarily disturbed) flow field. 


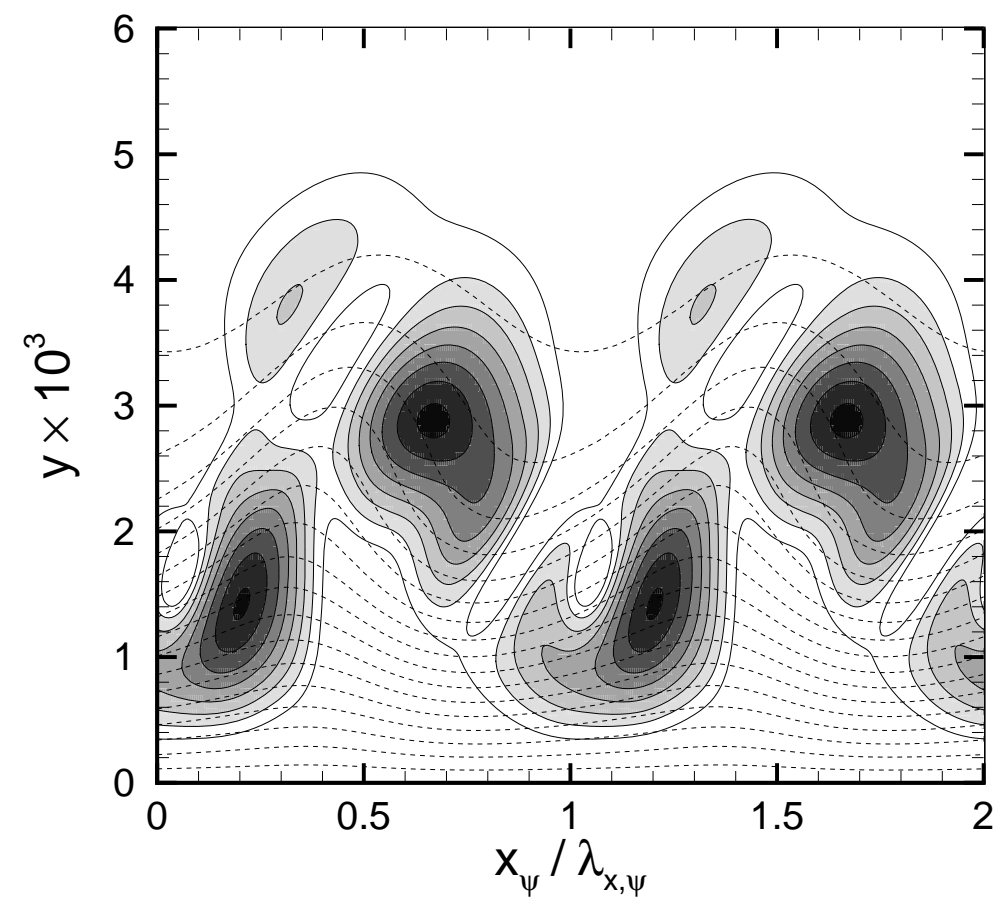

Figure 5.14: $\left|\bar{w}_{\psi}\right|$-isolines of secondary disturbance (mode 2) with $b_{\psi}=108.1$ at $x_{c}=0.65\left(N_{s}=12\right)$. The dashed lines indicate the $W_{\psi}$-component of the total (primarily disturbed) flow field.

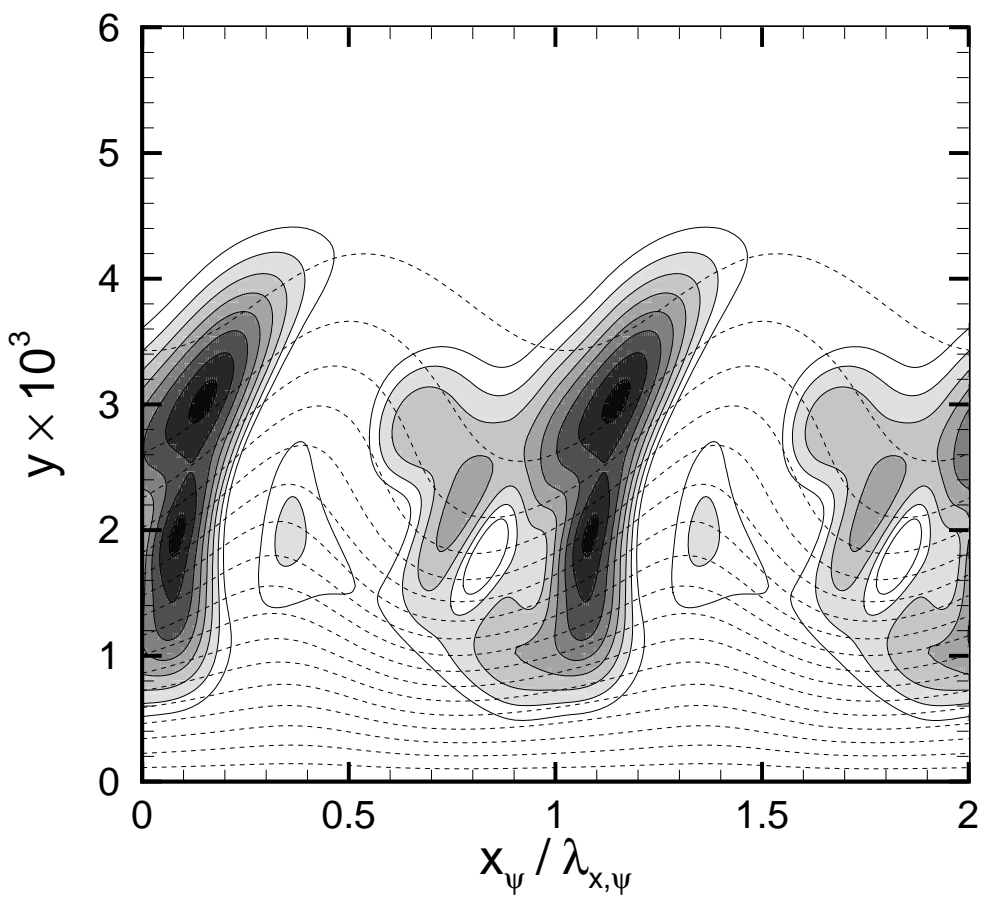

Figure 5.15: $\left|\bar{w}_{\psi}\right|$-isolines of secondary disturbance (mode 4) with $b_{\psi}=201.3$ at $x_{c}=0.65\left(N_{s}=12\right)$. The dashed lines indicate the $W_{\psi}$-component of the total (primarily disturbed) flow field. 


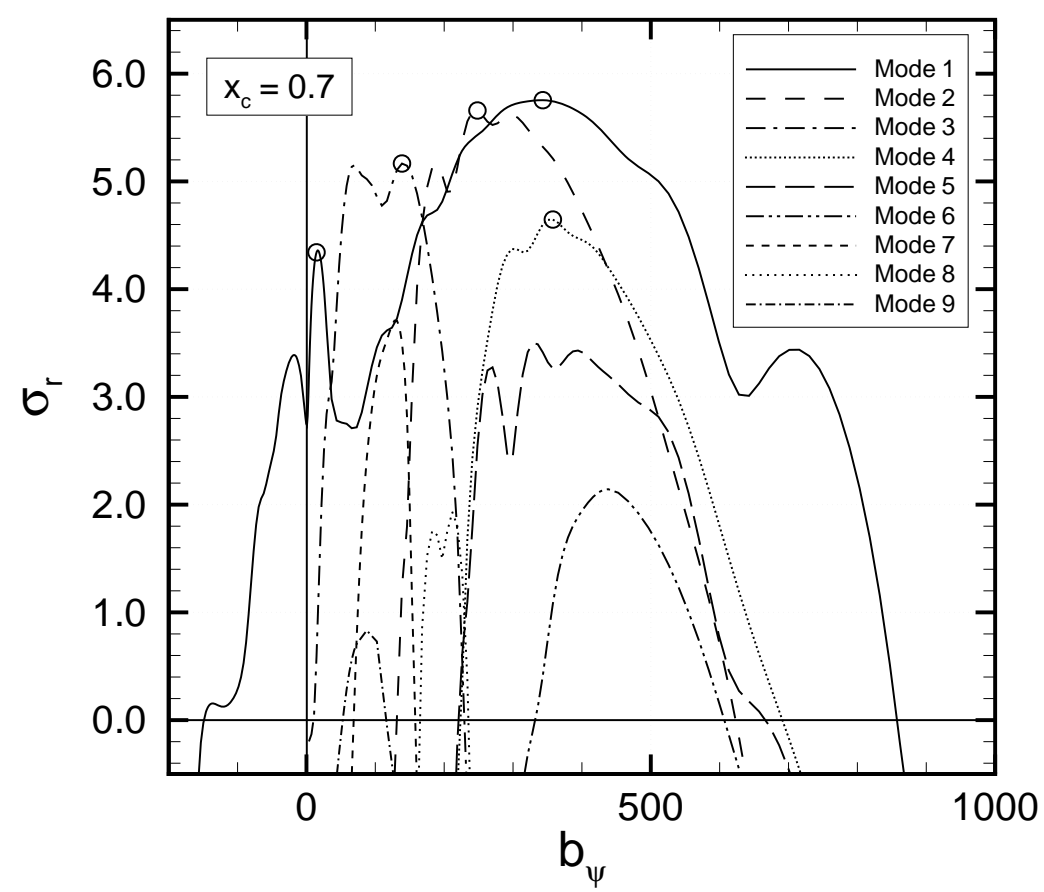

Figure 5.16: Temporal growth rate $\sigma_{r}$ of secondary disturbance modes versus wave number $b_{\psi}$ at $x_{c}=0.7\left(N_{s}=12\right)$. The circles indicate the locations, where the $\left|\bar{w}_{\psi}\right|$-isolines shown in figures $5.18-5.22$ have been extracted.

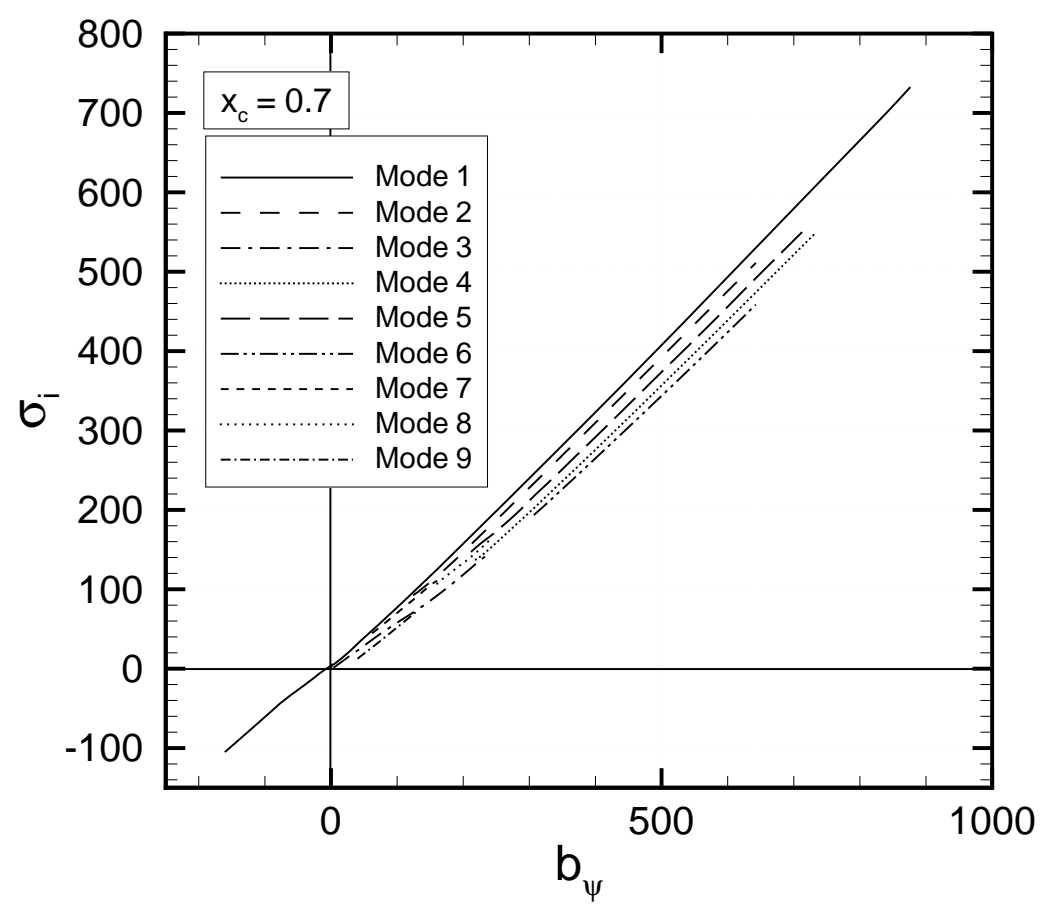

Figure 5.17: Angular frequency $\sigma_{i}$ of secondary disturbance modes versus wave number $b_{\psi}$ at $x_{c}=0.7\left(N_{s}=12\right)$. 


\begin{tabular}{||c|c|c|c|c|c||}
\hline \hline Mode & $b_{\psi}$ (maximal growth) & $\sigma_{r}$ & $\sigma_{i}$ & $f^{*}[\mathrm{~Hz}]$ & $\sigma_{i} / b_{\psi}$ \\
\hline \hline 1 & 343.2 & 5.75 & 275.7 & 1668 & 0.803 \\
\hline 1 & 14.6 & 4.34 & 12.2 & 74 & 0.836 \\
\hline 2 & 248.3 & 5.67 & 185.8 & 1123 & 0.748 \\
\hline 3 & 138.8 & 5.12 & 78.9 & 477 & 0.568 \\
\hline 4 & 357.8 & 4.65 & 242.3 & 1466 & 0.677 \\
\hline 5 & 328.6 & 3.50 & 234.3 & 1417 & 0.713 \\
\hline 6 & 438.2 & 2.14 & 294.8 & 1783 & 0.673 \\
\hline 7 & 131.4 & 3.71 & 91.8 & 555 & 0.699 \\
\hline 8 & 211.8 & 1.93 & 140.8 & 852 & 0.665 \\
\hline 9 & 87.3 & 0.83 & 43.6 & 264 & 0.499 \\
\hline \hline
\end{tabular}

Table 5.4: Properties of unstable secondary disturbance modes at $x_{c}=0.7$.

At its second amplification maximum of $\sigma_{r}=4.34$ at $b_{\psi}=14.6$ (here, the dimensional frequency $f^{*}=74 \mathrm{~Hz}$ ), the $\left|\bar{w}_{\psi}\right|$-isocontours of this mode look completely different. As can be seen from figure 5.19 , the amplitude distribution is similar to that of mode 1 at $x_{c}=0.65$. This indicates a possible kinship of this disturbance with traveling primary waves. Similar $\left|\bar{w}_{\psi}\right|^{-}$ isocontours are found for mode 3 for $b_{\psi}=138.8$ (figure 5.21) and mode 9 (not shown). Mode 3 has a dimensional frequency $f^{*}=477 \mathrm{~Hz}$, i.e. the frequencies of these disturbances are rather low in comparison with those of the other types of modes. The $\left|\bar{w}_{\psi}\right|$-distributions of mode 2 (figure 5.20) and mode 7 (not shown) exhibit a variety of local maxima. Hence, a clear classification of these disturbances is not possible.

Finally, a number of modes with the $\left|\bar{w}_{\psi}\right|$-maximum in the region of rather low shear can be observed. Figure 5.22 shows $\left|\bar{w}_{\psi}\right|$-isocontours of mode 4 for $b_{\psi}=357.8$. The structure of modes 5,6 , and 8 is very similar to that of mode 4 . A correlation between the location of the $\left|\bar{w}_{\psi}\right|$-maximum of these modes and inflection points of the primarily disturbed base flow could not be found. 


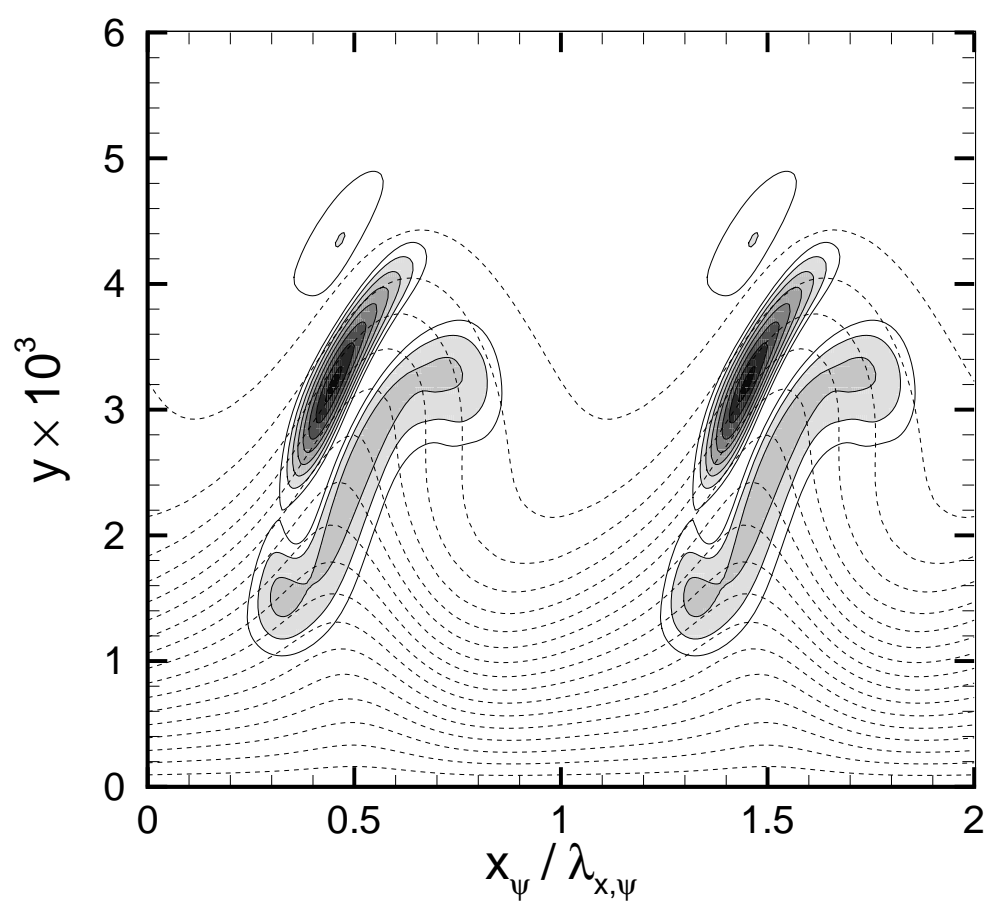

Figure 5.18: $\left|\bar{w}_{\psi}\right|$-isolines of secondary disturbance (mode 1) with $b_{\psi}=343.2$ at $x_{c}=0.7\left(N_{s}=12\right)$. The dashed lines indicate the $W_{\psi}$-component of the total (primarily disturbed) flow field.

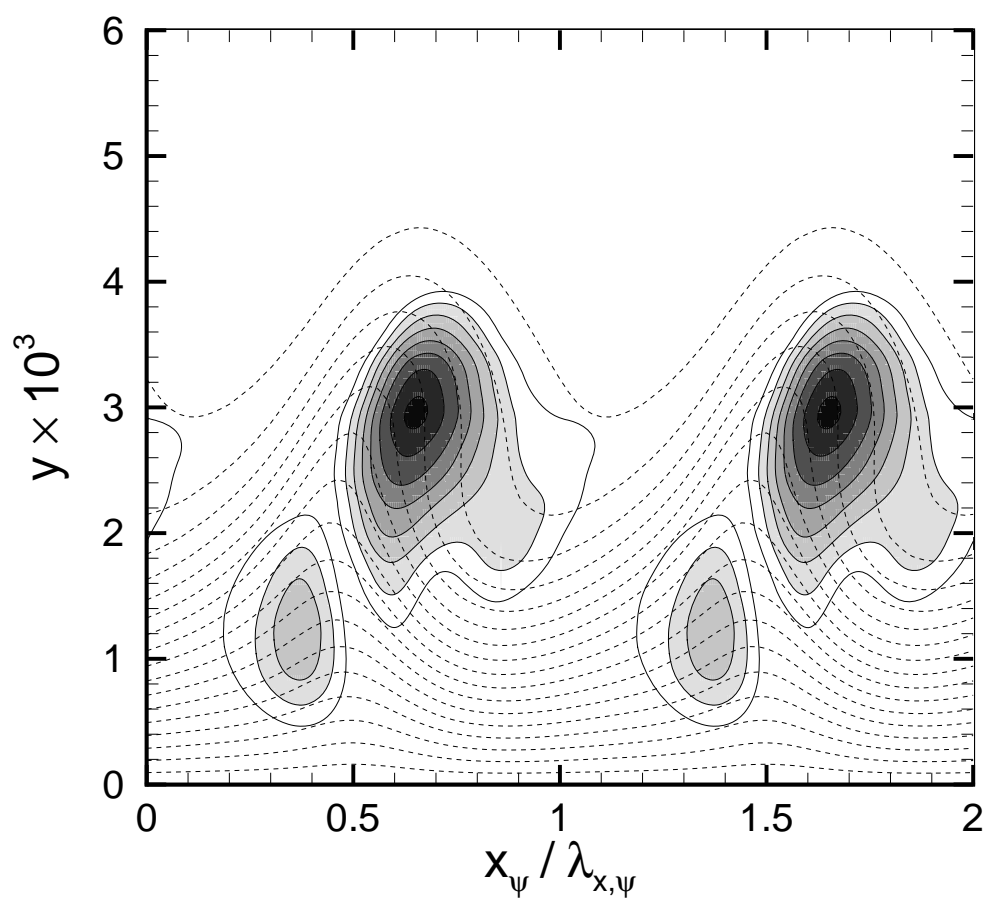

Figure 5.19: $\left|\bar{w}_{\psi}\right|$-isolines of secondary disturbance (mode 1 ) with $b_{\psi}=14.6$ at $x_{c}=0.7\left(N_{s}=12\right)$. The dashed lines indicate the $W_{\psi}$-component of the total (primarily disturbed) flow field. 


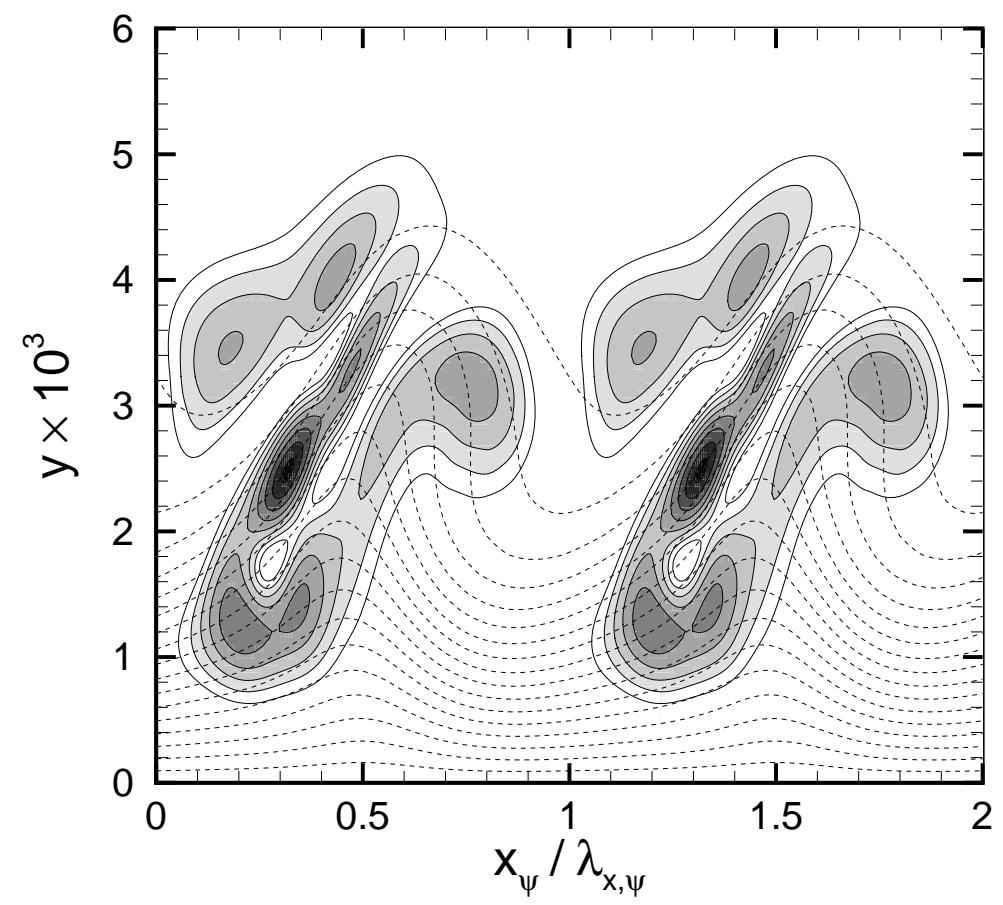

Figure 5.20: $\left|\bar{w}_{\psi}\right|$-isolines of secondary disturbance (mode 2) with $b_{\psi}=248.3$ at $x_{c}=0.7\left(N_{s}=12\right)$. The dashed lines indicate the $W_{\psi}$-component of the total (primarily disturbed) flow field.

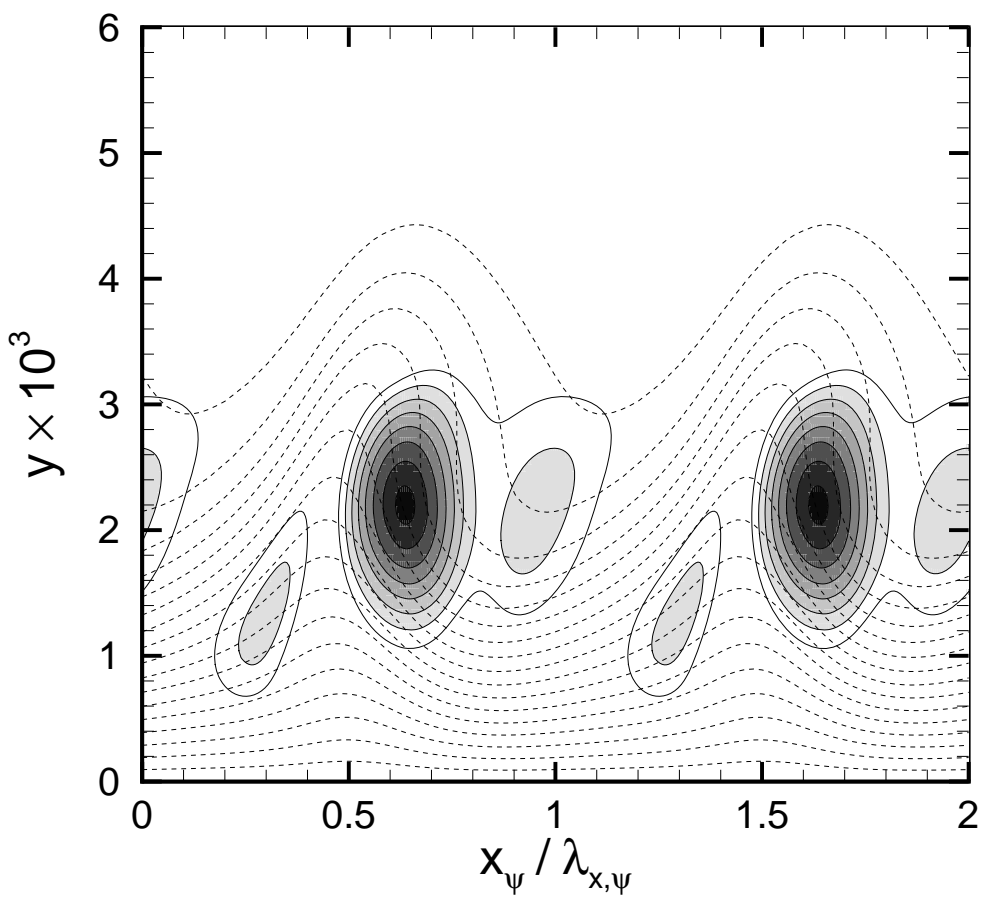

Figure 5.21: $\left|\bar{w}_{\psi}\right|$-isolines of secondary disturbance (mode 3 ) with $b_{\psi}=138.8$ at $x_{c}=0.7\left(N_{s}=12\right)$. The dashed lines indicate the $W_{\psi}$-component of the total (primarily disturbed) flow field. 


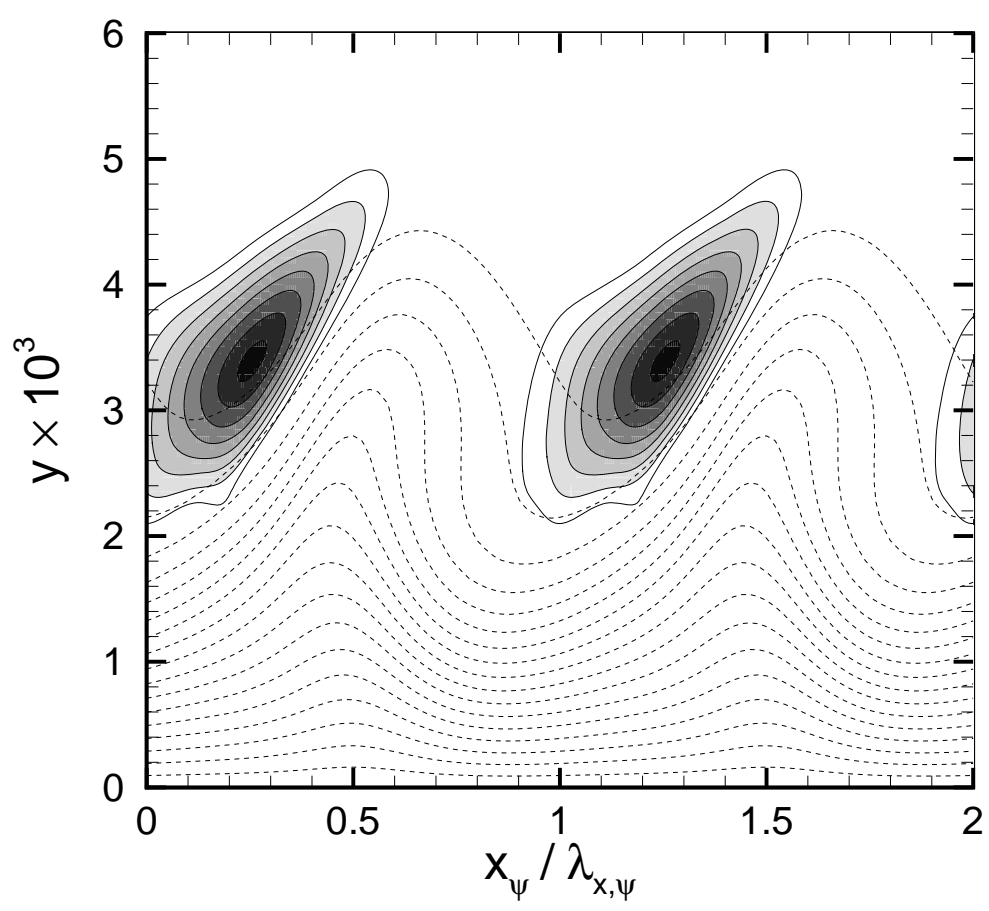

Figure 5.22: $\left|\bar{w}_{\psi}\right|$-isolines of secondary disturbance (mode 4) with $b_{\psi}=357.8$ at $x_{c}=0.7\left(N_{s}=12\right)$. The dashed lines indicate the $W_{\psi}$-component of the total (primarily disturbed) flow field. 


\subsubsection{Secondary Instability of a Strongly Distorted Base Flow}

At $x_{c}=0.75$, one secondary disturbance mode stands out clearly from the set of unstable modes, as shown in figure 5.23 . The square symbols in this figure depict the growth rates of unstable modes as found in global analyses for different wave numbers $b_{\psi}$. It can be seen that, while a large variety of modes is unstable at small values of $b_{\psi}$, for $b_{\psi}>900$, only one unstable mode exists. At $b_{\psi}=847.2$, the temporal growth rate of this mode exhibits a maximum, and the frequency of the mode is $f^{*}=4439 \mathrm{~Hz}$. For these parameters, $\left|\bar{w}_{\psi}\right|$-isocontours are shown in figure 5.24 . It can be seen, that this mode, which at $x_{c}=0.75$ by far exceeds all other modes in temporal growth, clearly is of "mode I"-type. While all other results depicted in figure 5.23 are obtained with $N_{s}=12$, the growth rate of the most unstable mode has also been computed with higher resolution $\left(N_{s}=20\right)$. The comparison between the two curves obtained with different resolution indicates, that the number of normal modes necessary for full resolution in the secondary instability analysis increases with increasing $b_{\psi}$. While the growth rate of the considered mode obtained with $N_{s}=20$ agrees very well with that obtained with $N_{s}=12$ for $b_{\psi}<500$, small deviations can be observed for higher values of $b_{\psi}$.

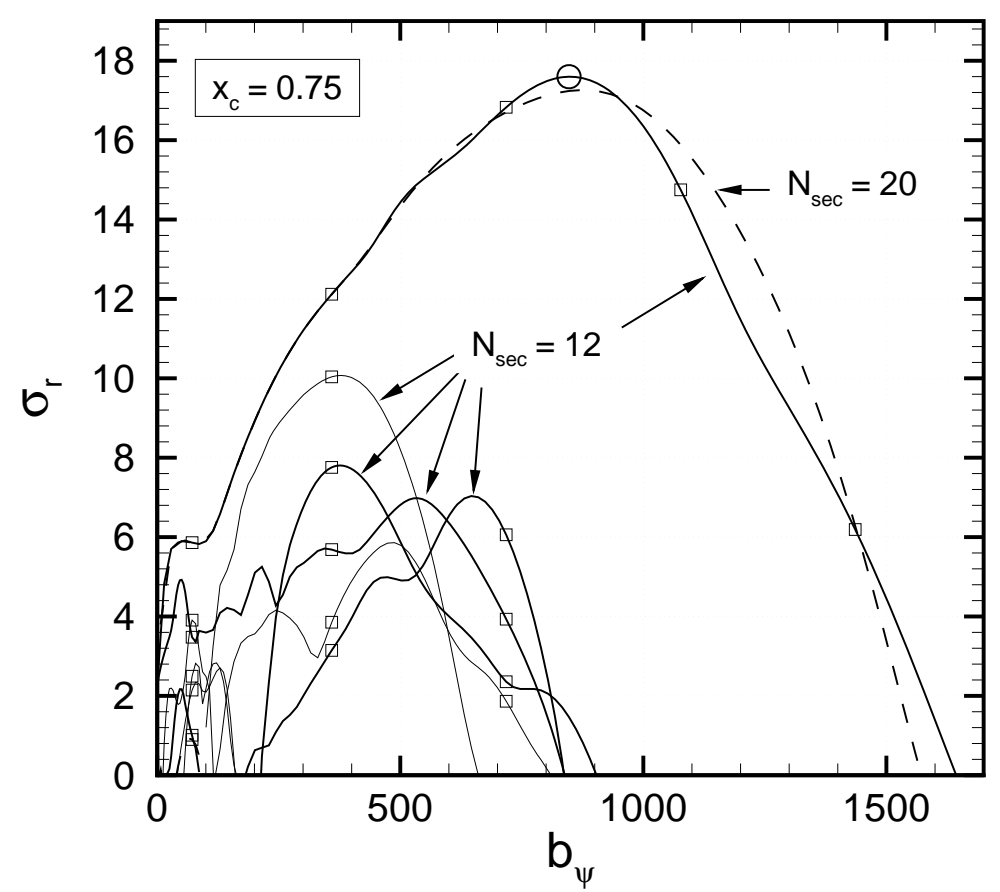

Figure 5.23: Temporal growth rate $\sigma_{r}$ of secondary disturbance modes versus wave number $b_{\psi}$ at $x_{c}=0.75\left(N_{s}=12\right)$. The circle indicates the location, where the $\left|\bar{w}_{\psi}\right|$-isolines shown in figure 5.24 have been extracted. Square symbols depict the growth rates of unstable modes as found in global analyses for different wave numbers $b_{\psi}$. 


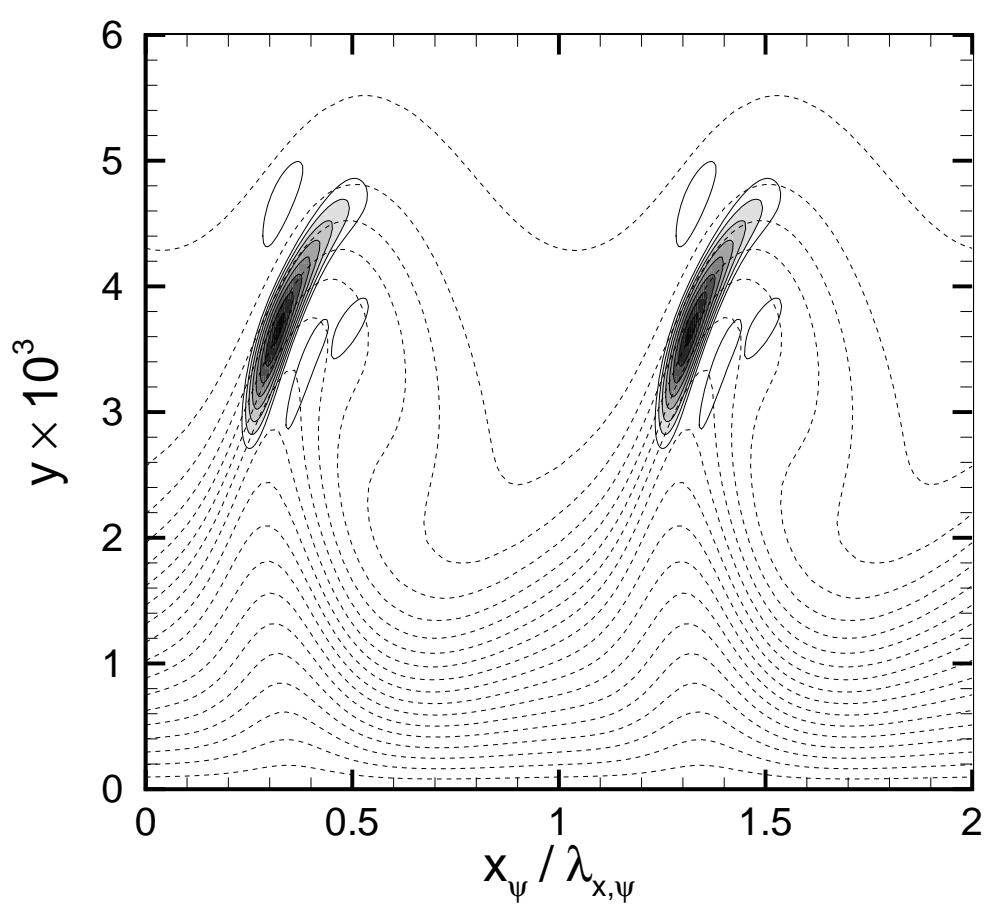

Figure 5.24: $\left|\bar{w}_{\psi}\right|$-isolines of secondary disturbance with $b_{\psi}=847.2$ at $x_{c}=$ $0.75\left(N_{s}=12\right)$. The dashed lines indicate the $W_{\psi}$-component of the total (primarily disturbed) flow field.

At $x_{c}=0.8$, the temporal growth rate of the most unstable secondary disturbance is $\sigma_{r}=43.8$. This is more than ten times the growth rate of the mode corresponding to a primary traveling crossflow wave as found at $x_{c}=0.4$ (see figure 5.2). The wave number of this mode is $b_{\psi}=1199$, the dimensional frequency is $f^{*}=6418 \mathrm{~Hz}$. Figure 5.25 shows the growth rate $\sigma_{r}$ of this mode for different values of $N_{s}$ and indicates that, for $N_{s}=16$ (this is equivalent to 33 normal modes), the growth rate does no longer depend on the number of normal modes. Furthermore, $N_{s}=16$ is sufficient to resolve the eigenfunctions of the corresponding secondary disturbance. While all other computations presented in this chapter are performed using a wallnormal resolution of $K=55$ collocation points, the growth rate $\sigma_{r}$ of the secondary disturbance with $b_{\psi}=1199$ has also been computed (for $N_{s}=16$ ) with $K=85$ collocation points. The result is included in figure 5.25 and agrees very well with the corresponding result obtained using $K=55$ collocation points. The latter resolution in wallnormal direction is, hence, considered to be sufficient. In combination with $K=55$ collocation points in wallnormal direction, the consideration of 33 normal modes in the secondary analysis leads to memory requirements of about 600 Megabytes for the local Wielandt iteration. One step in the Wielandt iteration takes about 30 minutes on a Sun Ultra-60 workstation, i.e. the analysis can be performed on a standard desktop workstation. However, the resolution can not be increased significantly, if such computers are to be used. 


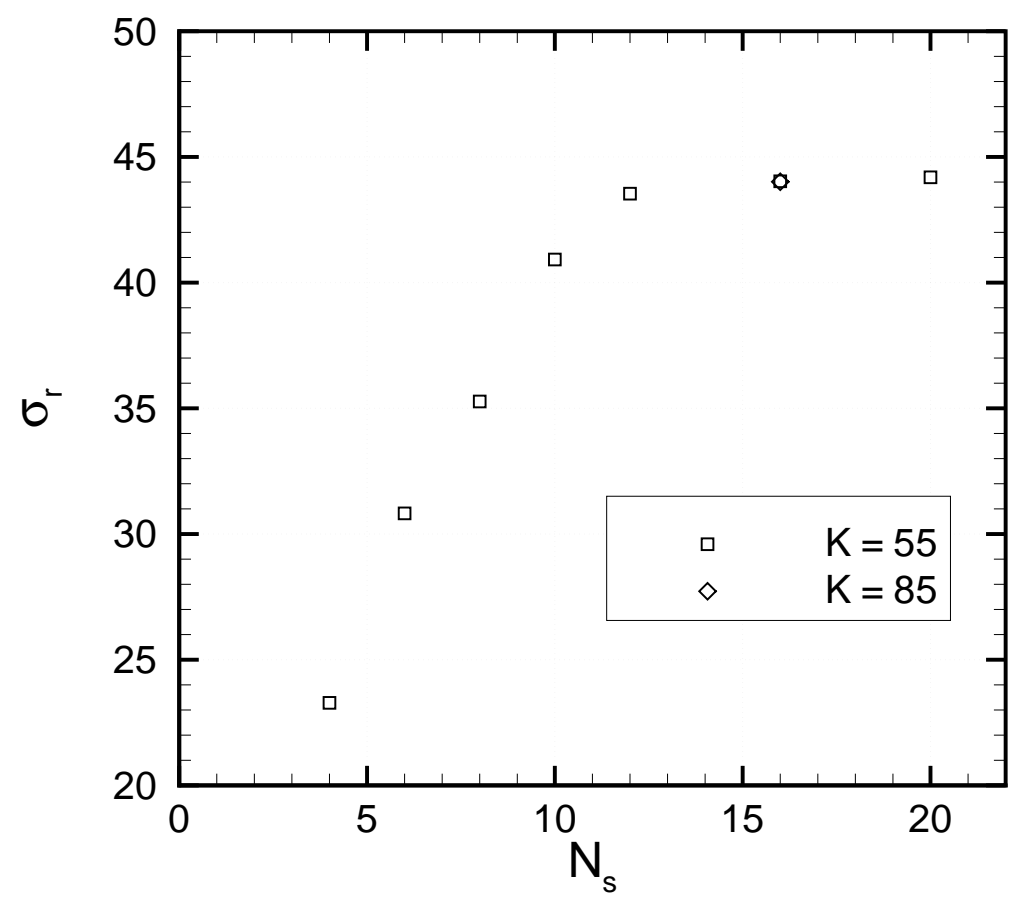

Figure 5.25: Temporal growth rate $\sigma_{r}$ of secondary disturbance with $b_{\psi}=$ 1199 at $x_{c}=0.8$ for varying number of normal modes and different wallnormal resolution.

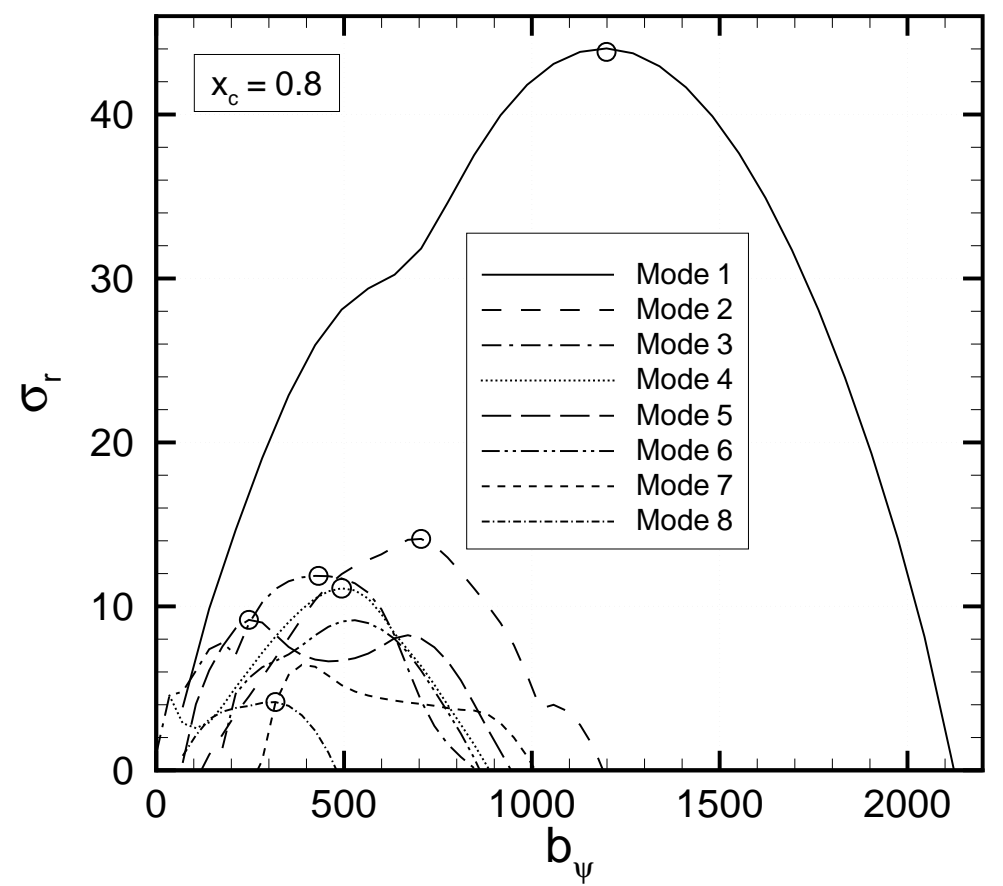

Figure 5.26: Temporal growth rate $\sigma_{r}$ of secondary disturbance modes versus wave number $b_{\psi}$ at $x_{c}=0.8\left(N_{s}=16\right)$. The circles indicate the locations, where the $\left|\bar{w}_{\psi}\right|$-isolines shown in the following figures have been extracted. 


\begin{tabular}{||c|c|c|c|c|c||}
\hline \hline Mode & $b_{\psi}$ (maximal growth) & $\sigma_{r}$ & $\sigma_{i}$ & $f^{*}[\mathrm{~Hz}]$ & $\sigma_{i} / b_{\psi}$ \\
\hline \hline 1 & 1198.9 & 43.82 & 1061.2 & 6418 & 0.885 \\
\hline 2 & 705.2 & 14.11 & 619.9 & 3749 & 0.879 \\
\hline 3 & 432.1 & 11.86 & 356.6 & 2157 & 0.825 \\
\hline 4 & 493.7 & 11.10 & 387.8 & 2346 & 0.785 \\
\hline 5 & 246.8 & 9.18 & 172.2 & 1042 & 0.698 \\
\hline 6 & 528.9 & 9.16 & 435.0 & 2631 & 0.822 \\
\hline 7 & 387.9 & 6.44 & 279.2 & 1689 & 0.720 \\
\hline 8 & 317.3 & 4.17 & 89.9 & 544 & 0.283 \\
\hline \hline
\end{tabular}

Table 5.5: Properties of unstable secondary disturbance modes at $x_{c}=0.8$.

Temporal growth rates of unstable modes are shown in figure 5.26. Circles mark the parameters of modes discussed in more detail below. Main properties of these modes are summarized in table 5.5. The mode, which clearly stands out from the set of unstable modes in terms of temporal growth rate $\sigma_{r}$, is depicted in figures 5.27 and 5.28 . As can be seen from the $\left|\bar{w}_{\psi}\right|$-isolines shown in figure 5.27 (top), this mode (mode 1) can be classified as "mode I"type disturbance. The maximum of the $\left|\bar{w}_{\psi}\right|$-distribution is located between $x_{\psi} / \lambda_{\psi}=0.3$ and $x_{\psi} / \lambda_{\psi}=0.4$ at the wallnormal distance $y=3.97 \cdot 10^{-3}$. For this wallnormal location, $\bar{w}_{\psi}$-isocontours in the wallparallel $x_{\psi}-z_{\psi}$-plane are depicted in figure 5.27 (bottom), which illustrates the restriction of the velocity fluctuations of the secondary disturbance to narrow regions "on the back" of the primary vortex. These facts are also illustrated in figure 5.28, where $\bar{w}_{\psi}$-isolines are shown in $x_{\psi}-y$-planes at different locations along the primary vortex (corresponding to different time points at some fixed location). The structure of the secondary disturbance is in good agreement with phase-averaged velocity profiles of high frequency secondary disturbances measured by Kawakami et al. [48], who studied the stability characteristics of stationary crossflow vortices with high amplitudes in the three-dimensional boundary layer on a swept plate (cf. Figure 17 in Kawakami et al. [48]).

The $\left|\bar{w}_{\psi}\right|$-maximum of mode 1 (see figure 5.27) correlates well with regions of high spanwise shear $\partial W_{\psi} / \partial x_{\psi}$ of the primarily distorted base flow as shown in figure 5.29 (cf. the detailed study of the relationship between secondary disturbance velocity fluctuations and shear layers in Görtler vortices by Bottaro and Klingmann [13]). However, the wallnormal shear, depicted in figure 5.30, also exhibits an extremum in this region. Even though the region of high wallnormal shear (i.e. the region, where "mode II"-type disturbances 

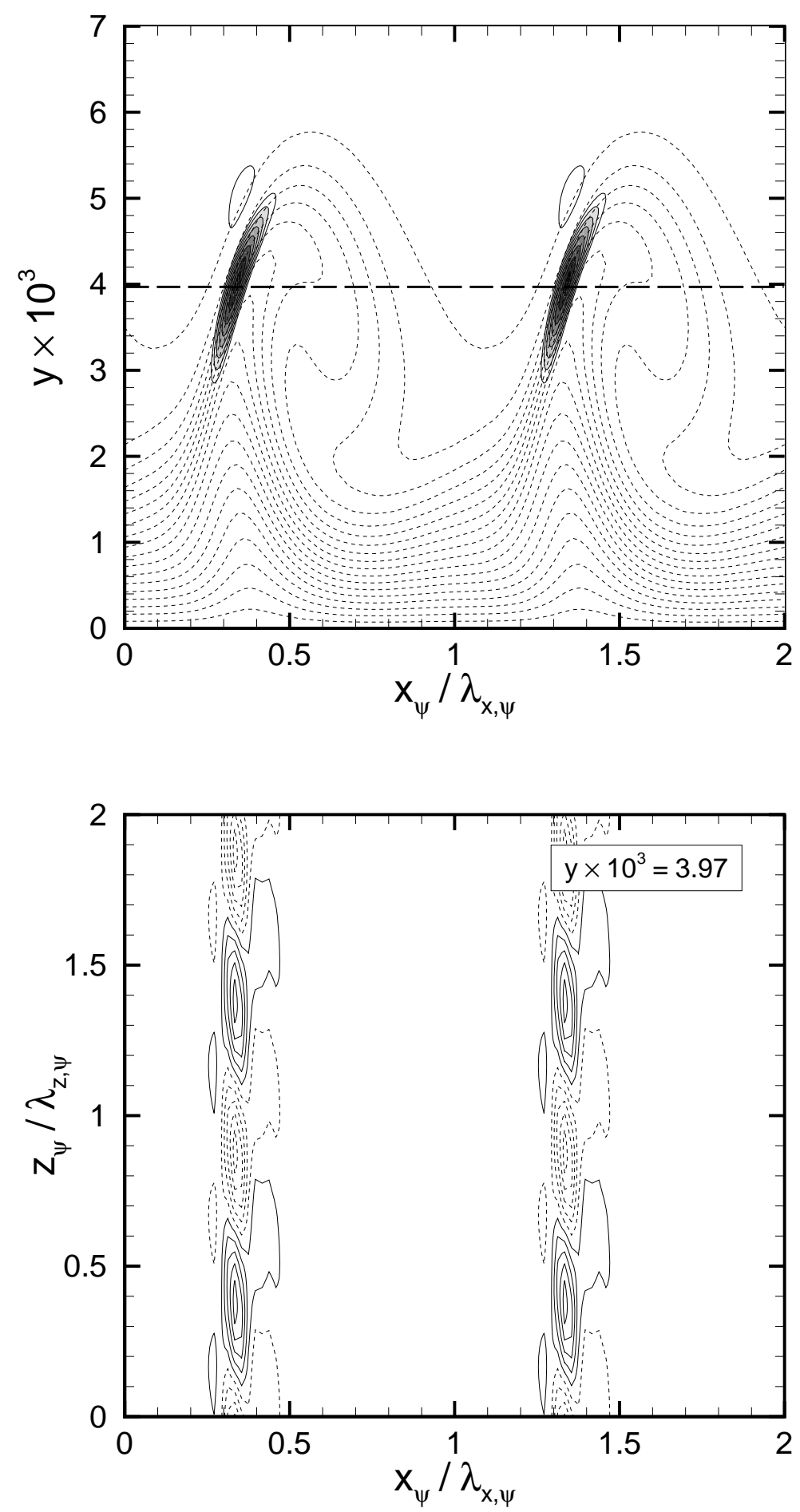

Figure 5.27: Top: $\left|\bar{w}_{\psi}\right|$-isolines of secondary disturbance (mode 1 ) with $b_{\psi}=$ 1199 at $x_{c}=0.8\left(N_{s}=16\right)$. The dashed lines indicate the $W_{\psi}$-component of the total (primarily disturbed) flow field. Bottom: $\bar{w}_{\psi}$-isolines in a wallparallel plane at distance $y=3.97 \cdot 10^{-3}$ from the wall, as indicated by the longdashed line in the top figure. 

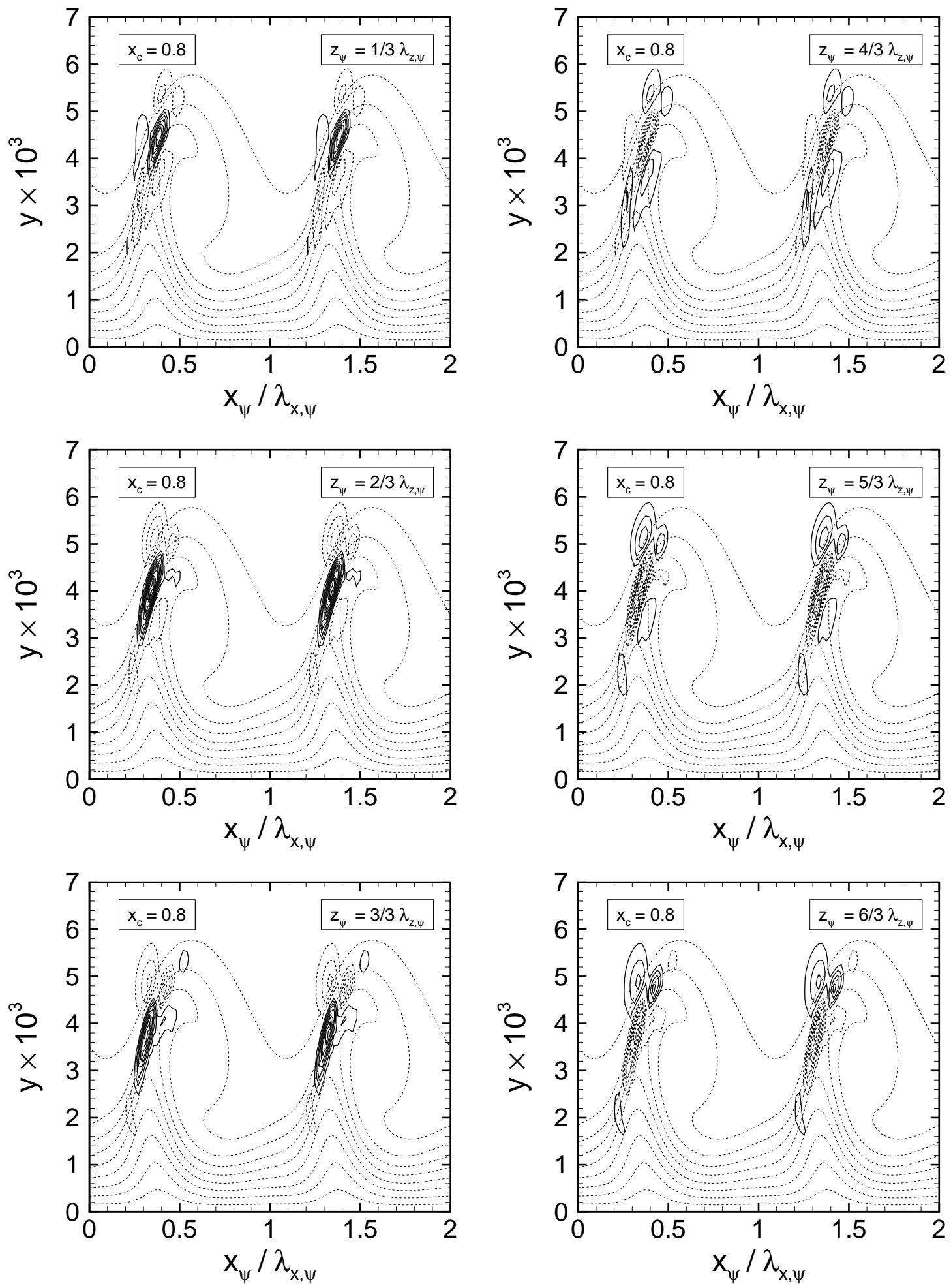

Figure 5.28: $\bar{w}_{\psi}$-isolines of secondary disturbance (mode 1) with $b_{\psi}=1199$ at $x_{c}=0.8\left(N_{s}=16\right)$ in $x_{\psi}-y$-planes at different location along the primary crossflow vortex. 
are expected) extends further away from the wall into the area "on top" of the primary crossflow vortex than the region of high spanwise shear, the large overlap of these two regions makes it rather difficult to clearly distinguish between these two causes of secondary instability and, hence, between the two types of disturbance modes.

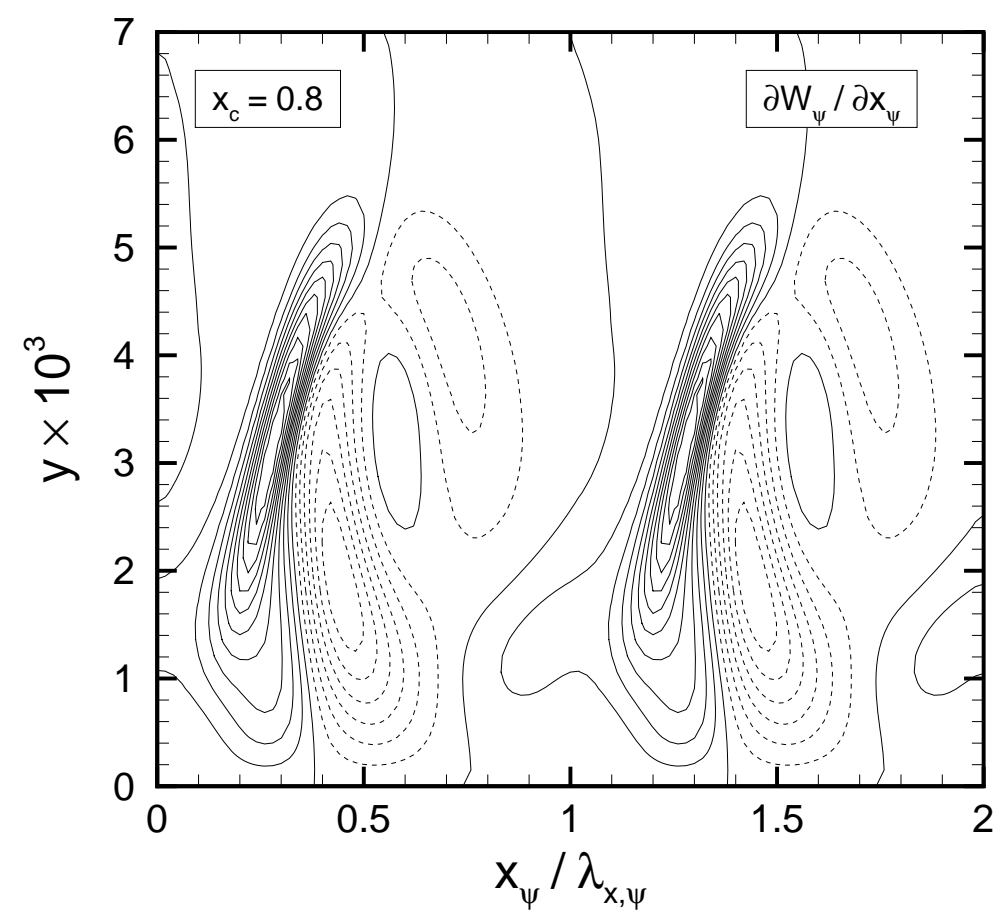

Figure 5.29: Iso-contours of $\partial W_{\psi} / \partial x_{\psi}$ at $x_{c}=0.80$.

As additional aid to study the properties of the primarily distorted base flow at $x_{c}=0.8$, spanwise $W_{\psi}$-profiles are shown in figure 5.31 for a variety of wallnormal distances. The low-speed region between $x_{\psi}=0.2$ and $x_{\psi}=0.5$ can be clearly seen in this plot. Figure 5.32 shows wallnormal $W_{\psi}$-profiles at different positions across the primary crossflow vortex. Especially the profiles at $x_{\psi} / \lambda_{\psi}=0.4, x_{\psi} / \lambda_{\psi}=0.5$, and $x_{\psi} / \lambda_{\psi}=0.6$ exhibit characteristic dips at $y \approx 4 \cdot 10^{-3}$. Above this point, comparatively high negative wallnormal shear can be seen (see also figure 5.30).

This region of high wallnormal shear $\partial W_{\psi} / \partial y$ correlates with the $\left|\bar{w}_{\psi}\right|^{-}$ maximum of the disturbance shown in figure 5.33. In the classification scheme used by Malik et al. [62] and by Koch et al. [53], this mode is therefore of "mode II"-type. Its wave number is $b_{\psi}=705.2$, and its dimensional frequency is $f^{*}=3749 \mathrm{~Hz}$. The temporal growth rate of this mode (mode 2 ) is less than one third of that of mode 1 . Still, mode 2 exhibits the second largest temporal growth rate at $x_{c}=0.8$.

It has already been mentioned that, due to the large overlap of regions with strong shear in $x_{\psi}$ - and $y$-direction, a strict distinction of secondary disturbances into "mode I"-type modes and "mode II"-type modes is not possible. 


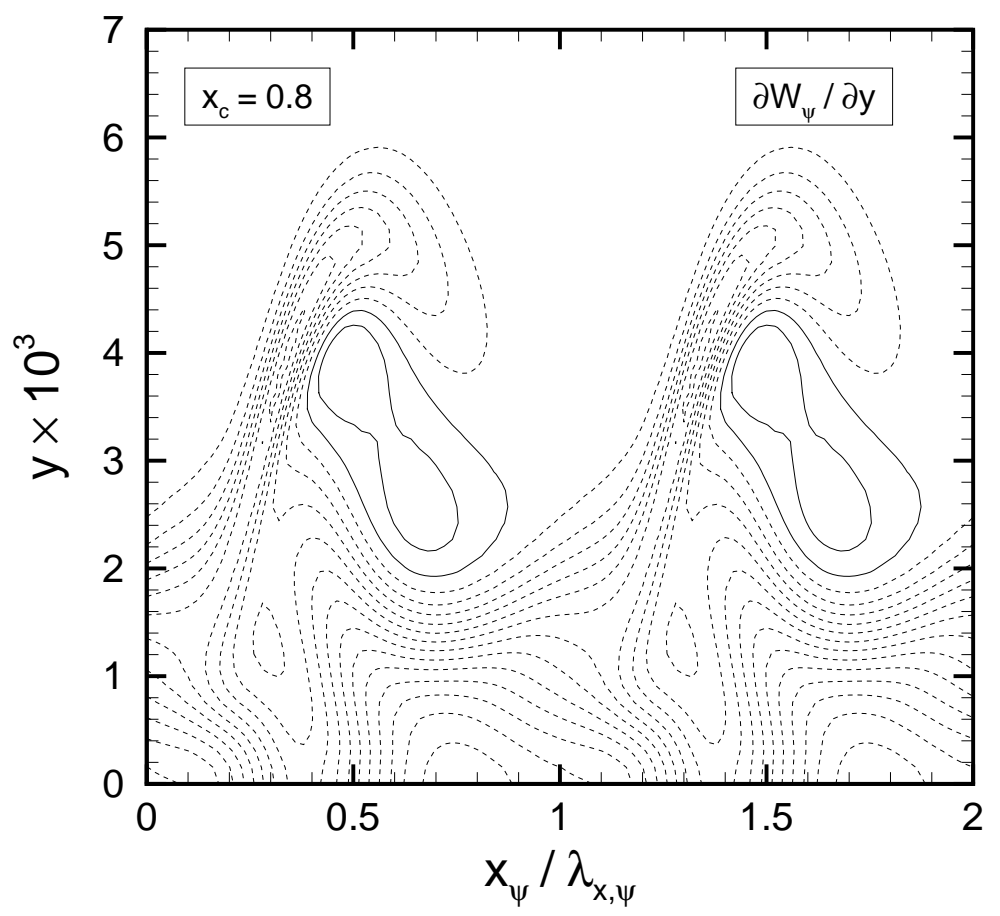

Figure 5.30: Iso-contours of $\partial W_{\psi} / \partial y$ at $x_{c}=0.80$.

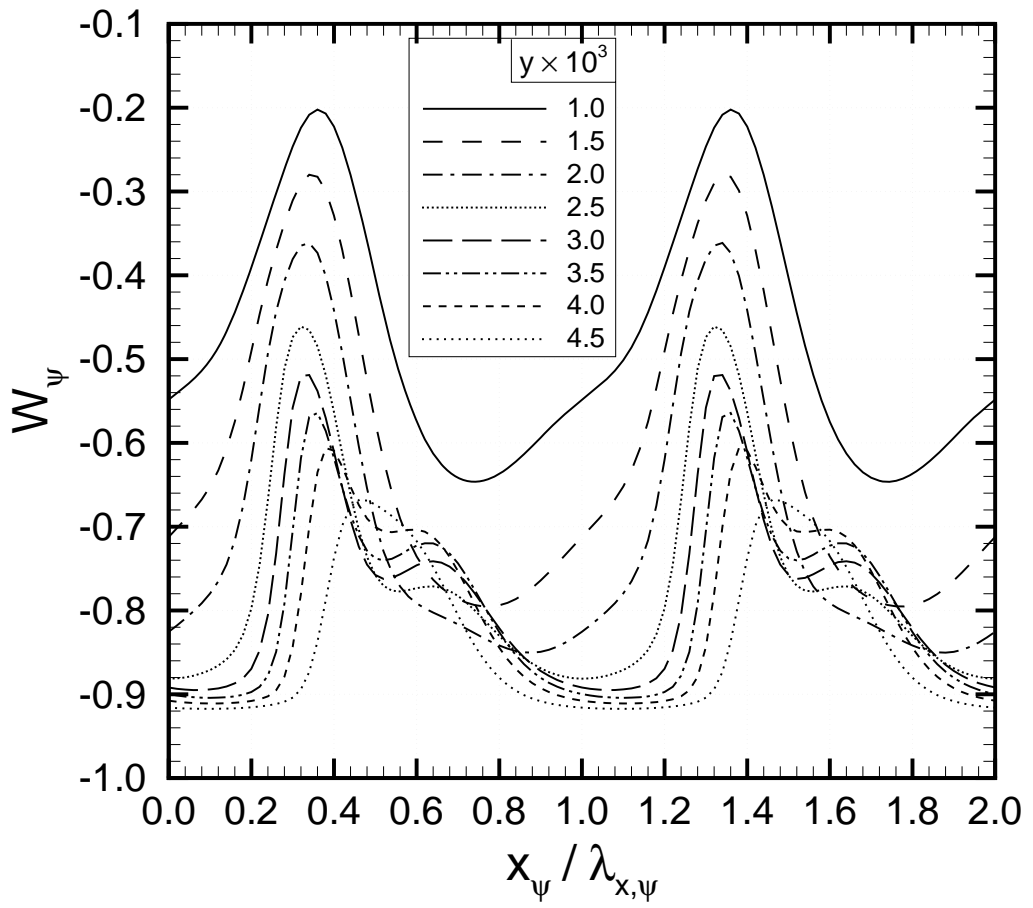

Figure 5.31: Variation of $W_{\psi}$ (primarily distorted flow field) along $x_{\psi}$ at $x_{c}=$ 0.8 and different distances from the wall. 


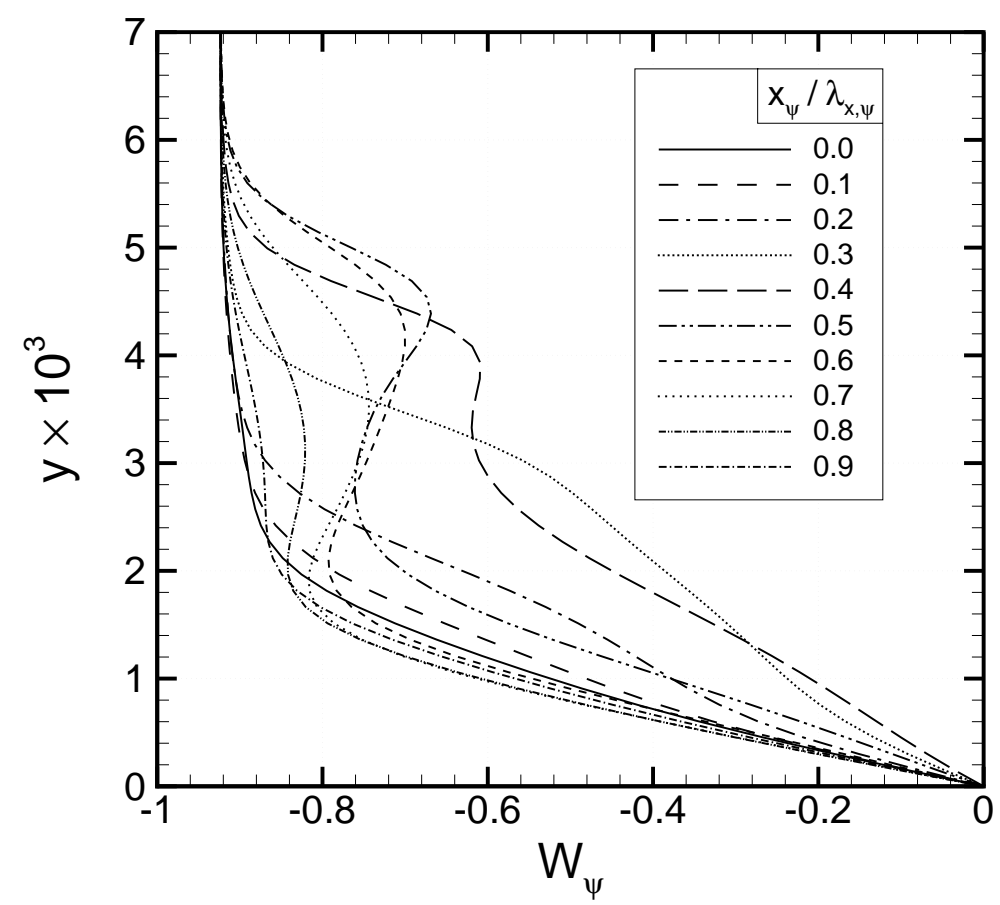

Figure 5.32: $W_{\psi}$-profiles at $x_{c}=0.8$ and different positions across the primary crossflow vortex.

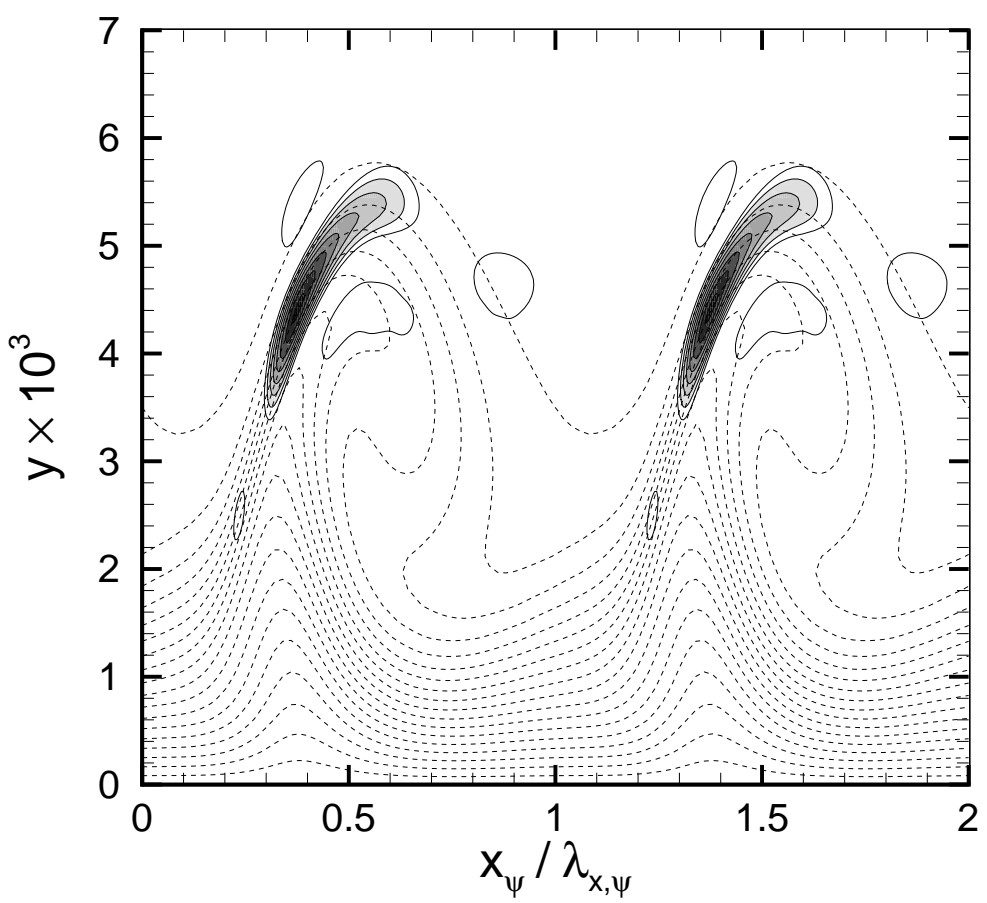

Figure 5.33: $\left|\bar{w}_{\psi}\right|$-isolines of secondary disturbance (mode 2) with $b_{\psi}=705.2$ at $x_{c}=0.8\left(N_{s}=16\right)$. The dashed lines indicate the $W_{\psi}$-component of the total (primarily disturbed) flow field. 


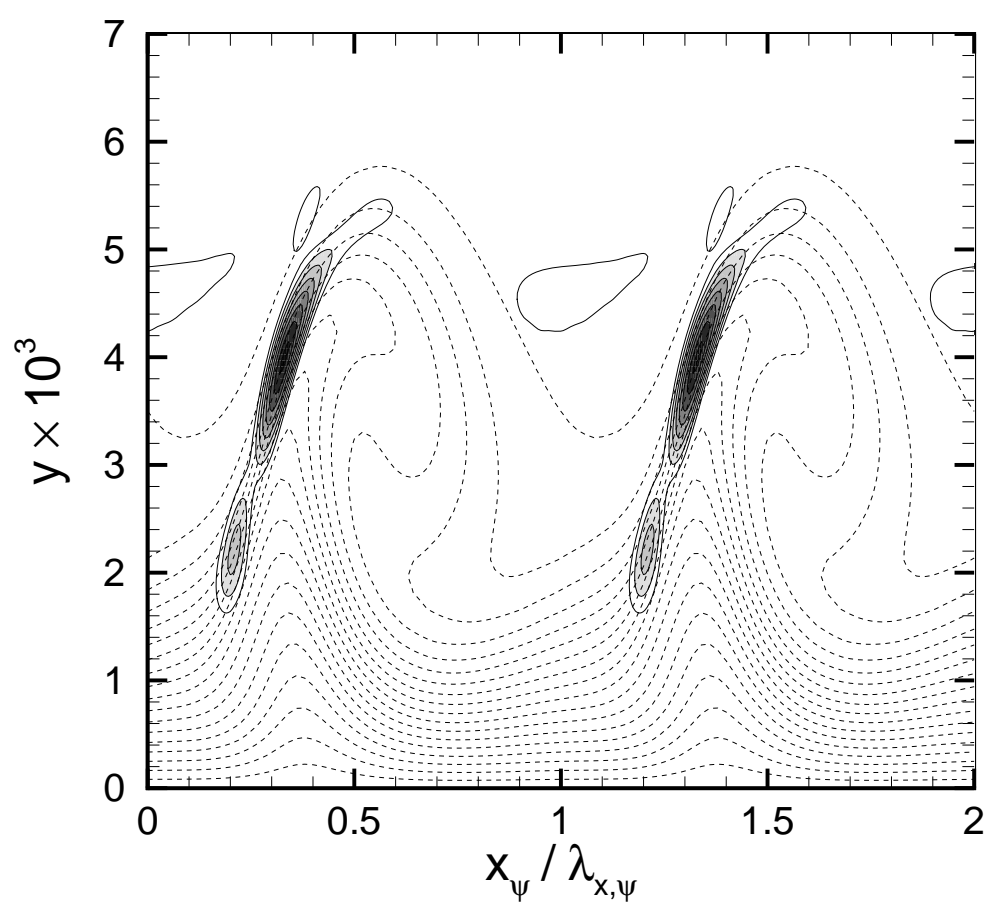

Figure 5.34: $\left|\bar{w}_{\psi}\right|$-isolines of secondary disturbance (mode 3 ) with $b_{\psi}=432.1$ at $x_{c}=0.8\left(N_{s}=16\right)$. The dashed lines indicate the $W_{\psi}$-component of the total (primarily disturbed) flow field.

The $\left|\bar{w}_{\psi}\right|$-isolines of mode 3 shown in figure 5.34 look similar to those of a "mode I"-type disturbance, but the $\left|\bar{w}_{\psi}\right|$-distribution also extends into the region associated with wallnormal shear of $W_{\psi}$ near the edge of the boundary layer.

The location of the $\left|\bar{w}_{\psi}\right|$-maxima of mode 4 (see figure 5.35) and mode 6 allows to class these modes with the "mode I"-category. However, both disturbances also exhibit local $\left|\bar{w}_{\psi}\right|$-maxima in the region of low shear "between" the primary vortices. Mode 5 (shown in figure 5.36) and mode 7 represent this class of secondary disturbances, the cause of which is still to be found, in pure culture. In figure 5.37, $\left|\bar{w}_{\psi}\right|$-isolines of mode 8 are shown. This mode is located rather near to the wall with a $\left|\bar{w}_{\psi}\right|$-maximum at $y \approx 0.8 \cdot 10^{-3}$. Its maximal growth rate is $\sigma_{r}=4.17$ for $b_{\psi}=317.3$. Due to the comparatively small growth rate of this mode, it can easily be overlooked in the numerical analysis. Because of its location near to the wall, this mode might, however, play a role in experiments, where secondary disturbances are triggered in the near-wall region.

At $x_{c}=0.95$, the $\left|\tilde{u}_{c}\right|_{\text {max }}$-amplitude of the fundamental primary vortex $(0,-1,1)$ has reached $23.1 \%$, the mean flow distortion has grown to $\left|\tilde{u}_{c}\right|_{\max }=$ $14.2 \% . W_{\psi}$-profiles across the primary vortex are shown in figure 5.38 for different distances from the wall. As at $x_{c}=0.8$, a prominent low-speed region can be observed between $x_{\psi} / \lambda_{\psi}=0.2$ and $x_{\psi} / \lambda_{\psi}=0.5$. The highly distorted wallnormal $W_{\psi}$-profiles are shown in figure 5.39 for different po- 


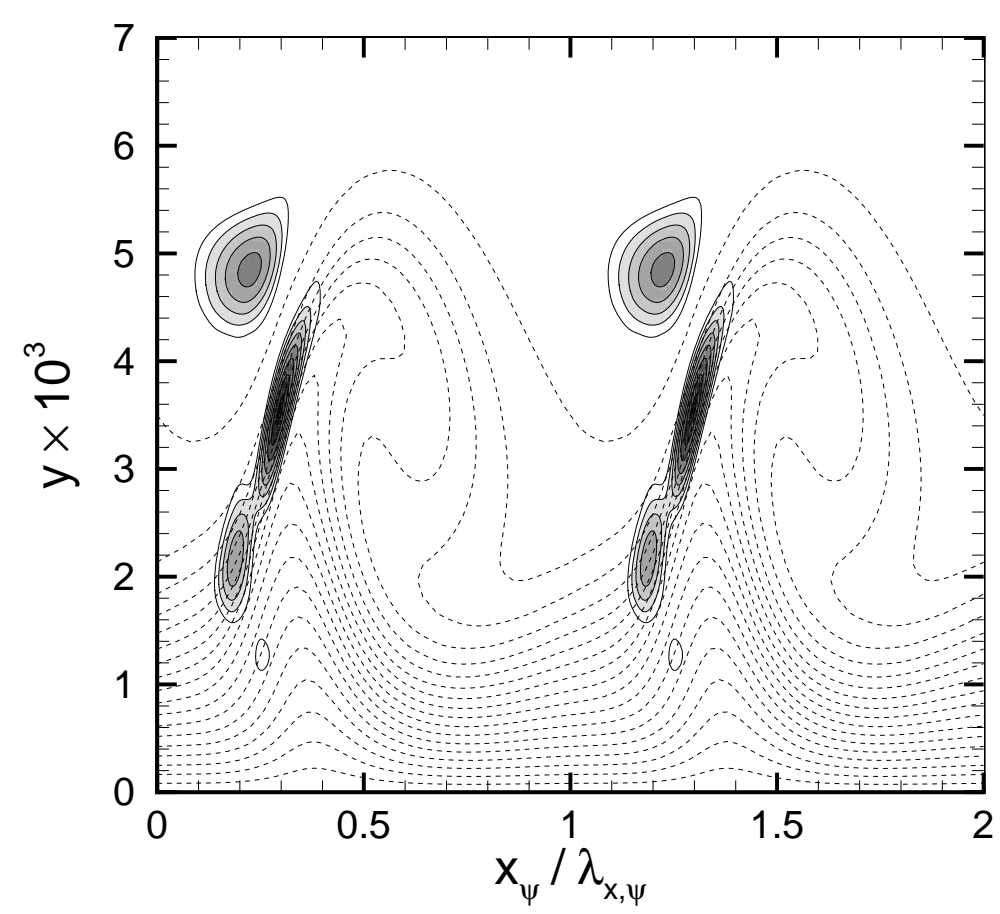

Figure 5.35: $\left|\bar{w}_{\psi}\right|$-isolines of secondary disturbance (mode 4) with $b_{\psi}=493.7$ at $x_{c}=0.8\left(N_{s}=16\right)$. The dashed lines indicate the $W_{\psi}$-component of the total (primarily disturbed) flow field.

sitions across the primary vortex. The characteristic dip between $x_{\psi} / \lambda_{\psi}=0.4$ and $x_{\psi} / \lambda_{\psi}=0.6$ has moved further away from the wall to $y \approx 6.5 \cdot 10^{-3}$. The spanwise derivative $\partial W_{\psi} / \partial x_{\psi}$ and the wallnormal derivative $\partial W_{\psi} / \partial y$ of the primarily disturbed base flow are summarized in the contour plots shown in figure 5.40 and figure 5.41, respectively.

The secondary instability analysis at $x_{c}=0.95$ has been performed with $K=55$ collocation points in wallnormal direction and $N_{s}=16$, since, as described above, an essential increase in resolution would exceed the capabilities of up-to-date desktop workstations. Especially for large values of $b_{\psi}$, this resolution might not be quite sufficient, but it is adequate to compute good estimates for growth rates and velocity profiles of unstable secondary disturbances. The growth rates of ten unstable modes are summarized in figure 5.42. $\left|\bar{w}_{\psi}\right|$-isolines of the disturbances marked by circles are shown in figures 5.43 - 5.49; main properties of these disturbances are (ordered by growth rate) listed in table 5.6

Mode $1\left(\left|\bar{w}_{\psi}\right|\right.$-isolines of this mode are shown in figure 5.43$)$ is by far the most unstable mode. Its temporal growth rate is $\sigma_{r}=65.2$, which is $50 \%$ higher than the highest growth rate found at $x_{c}=0.8$. This mode can be clearly correlated with regions of high spanwise shear $\partial W_{\psi} / \partial x_{\psi}$, and, thus, it is of type "mode I". Its dimensional frequency is $f^{*}=5768 \mathrm{~Hz}$. The maximal growth rate of mode 2 is $\sigma_{r}=31.3$, i.e. about half as high as the maximal growth rate of mode 1 . In comparison with mode 1 , the $\left|\bar{w}_{\psi}\right|$-maximum of 

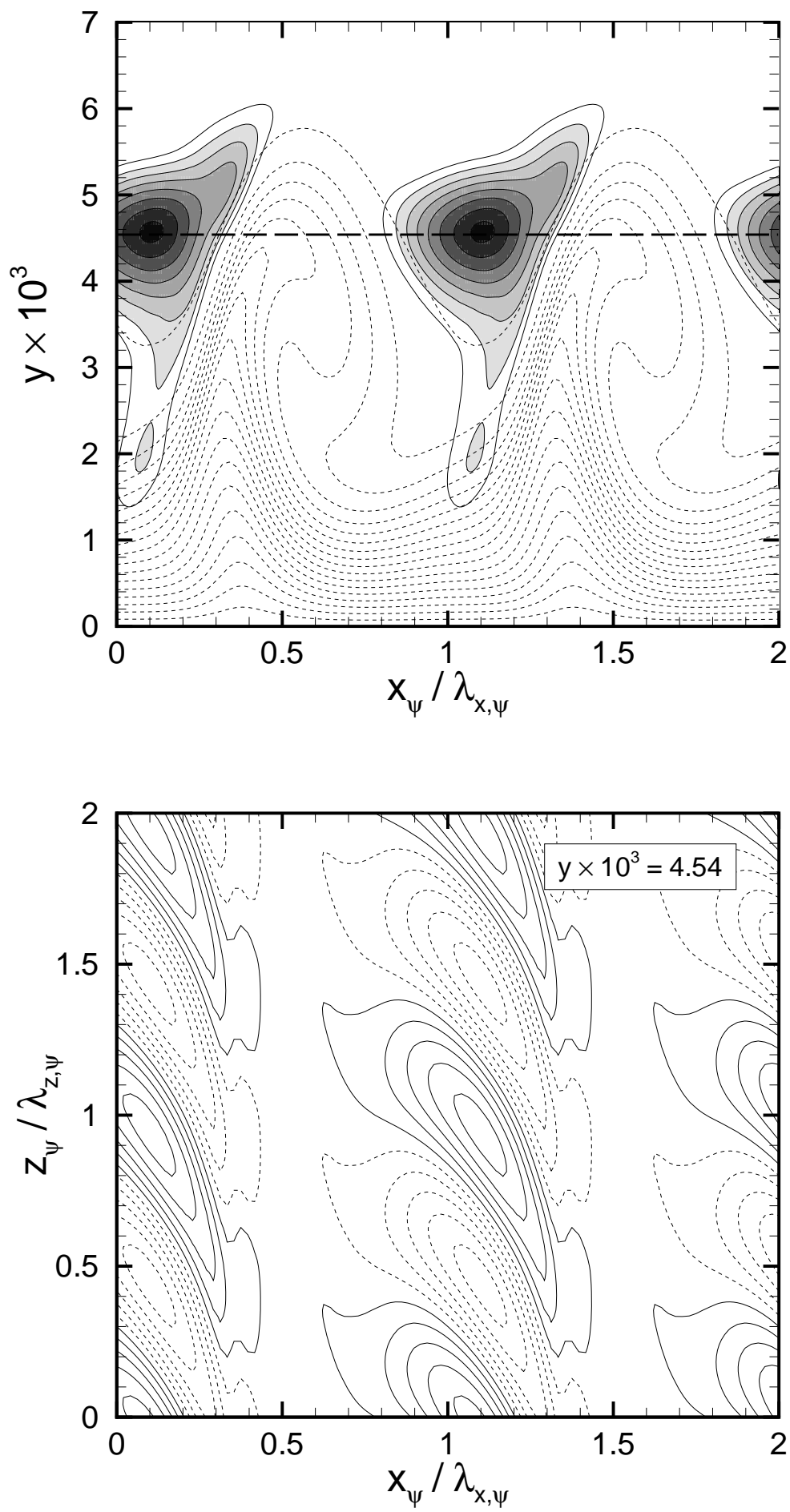

Figure 5.36: Top: $\left|\bar{w}_{\psi}\right|$-isolines of secondary disturbance (mode 5) with $b_{\psi}=$ 246.8 at $x_{c}=0.8\left(N_{s}=16\right)$. The dashed lines indicate the $W_{\psi}$-component of the total (primarily disturbed) flow field. Bottom: $\bar{w}_{\psi}$-isolines in a wallparallel plane at distance $y=4.54 \cdot 10^{-3}$ from the wall, as indicated by the long-dashed line in the top figure. 

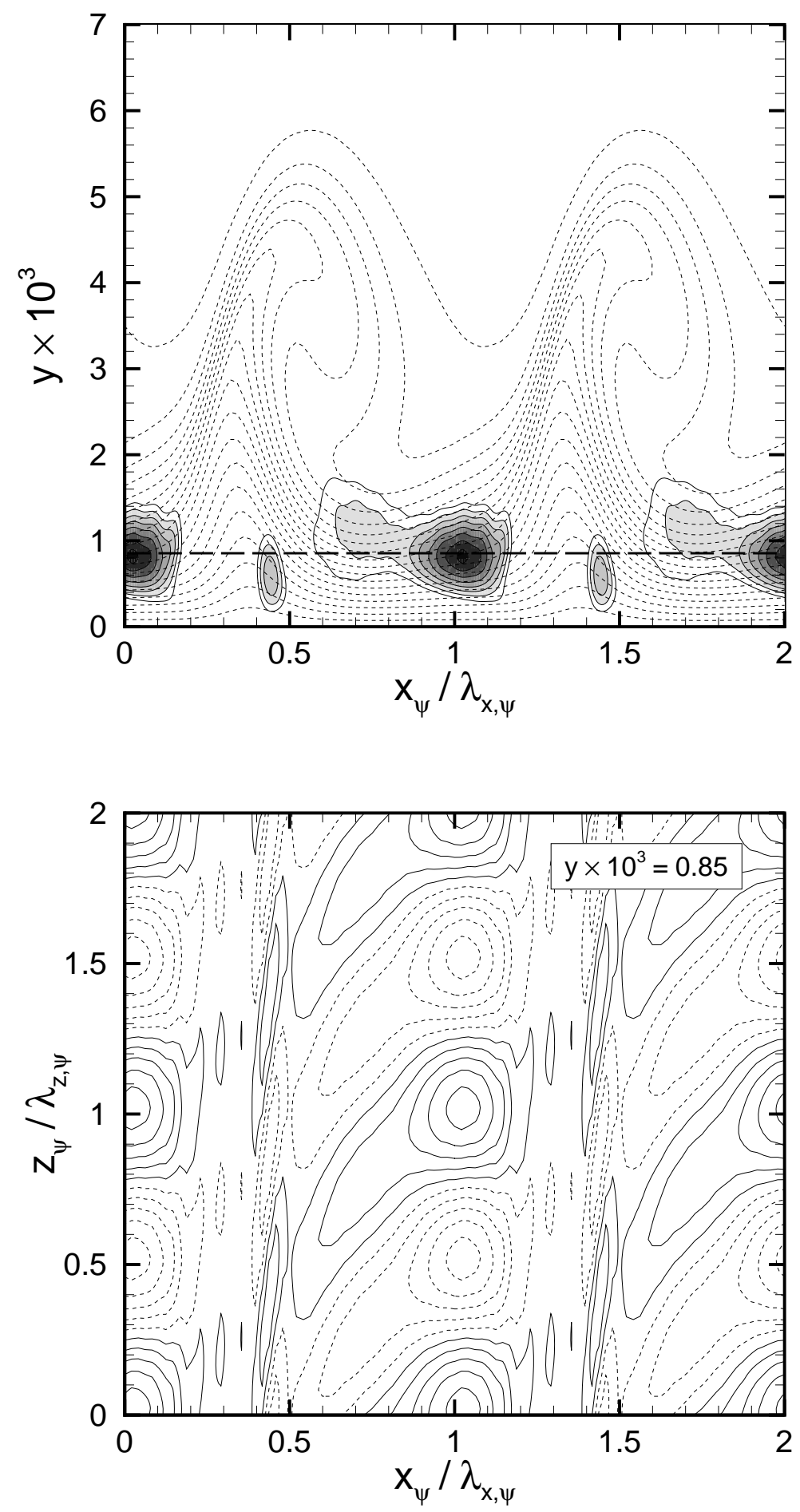

Figure 5.37: Top: $\left|\bar{w}_{\psi}\right|$-isolines of secondary disturbance (mode 8) with $b_{\psi}=$ 317.3 at $x_{c}=0.8\left(N_{s}=16\right)$. The dashed lines indicate the $W_{\psi}$-component of the total (primarily disturbed) flow field. Bottom: $\bar{w}_{\psi}$-isolines in a wallparallel plane at distance $y=0.85 \cdot 10^{-3}$ from the wall, as indicated by the long-dashed line in the top figure. 


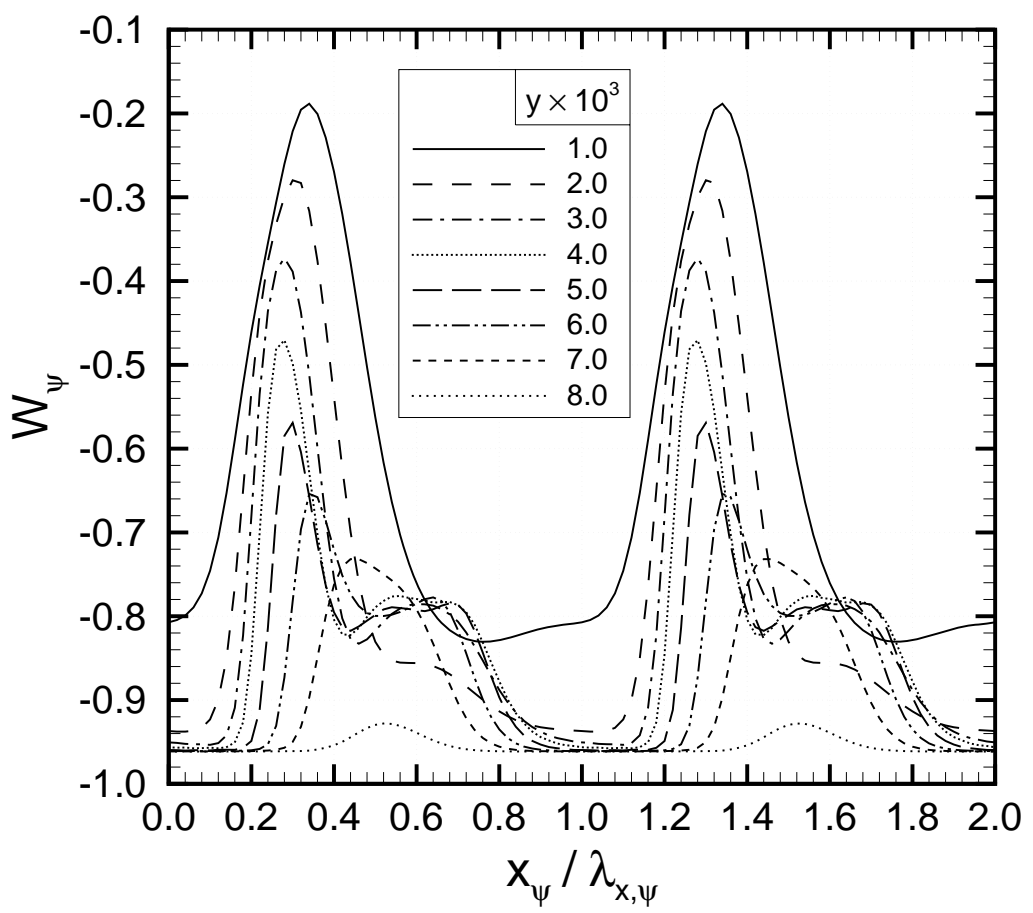

Figure 5.38: Variation of $W_{\psi}$ (primarily distorted flow field) along $x_{\psi}$ at $x_{c}=$ 0.95 and different distances from the wall.

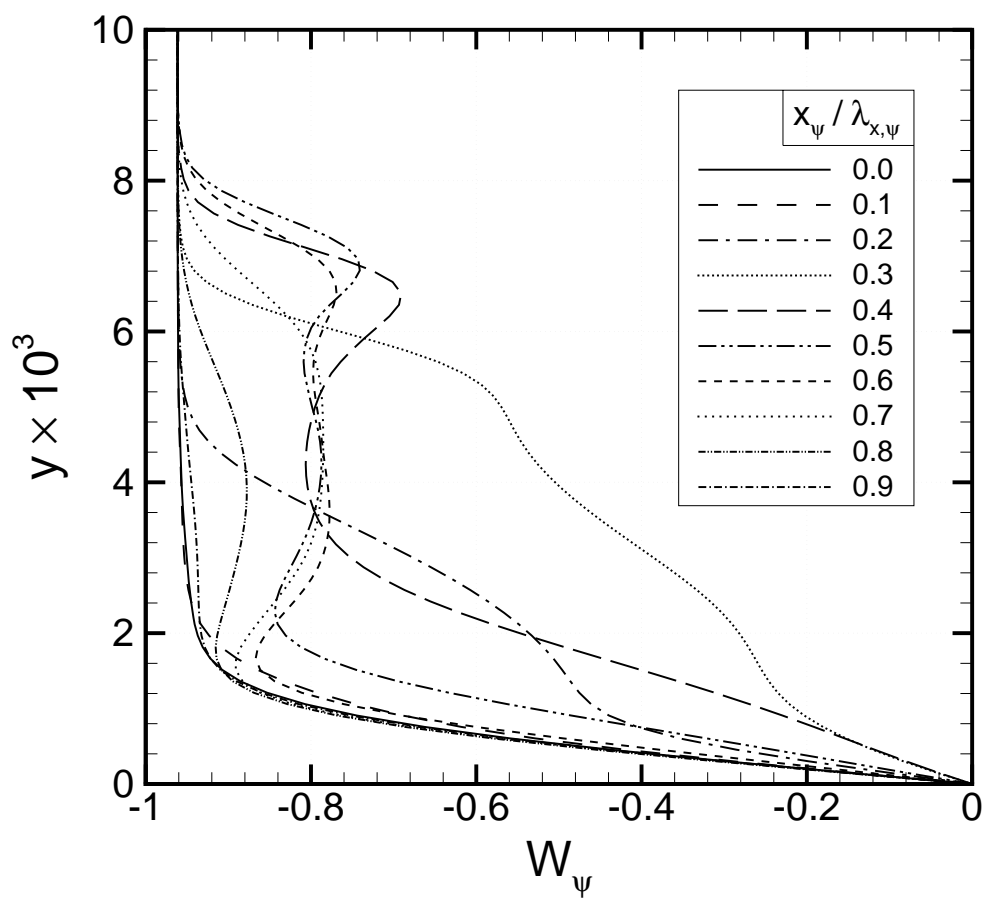

Figure 5.39: $W_{\psi}$-profiles at $x_{c}=0.95$ and different positions across the primary crossflow vortex. 


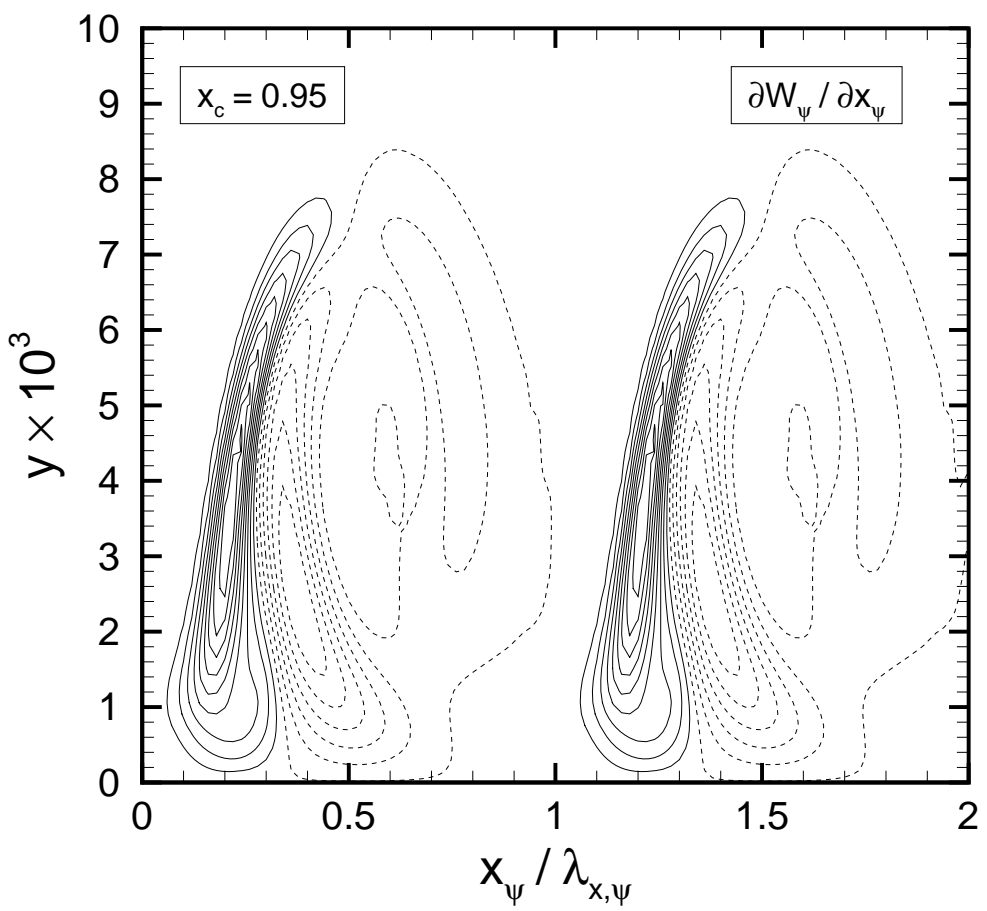

Figure 5.40: Iso-contours of $\partial W_{\psi} / \partial x_{\psi}$ at $x_{c}=0.95$.

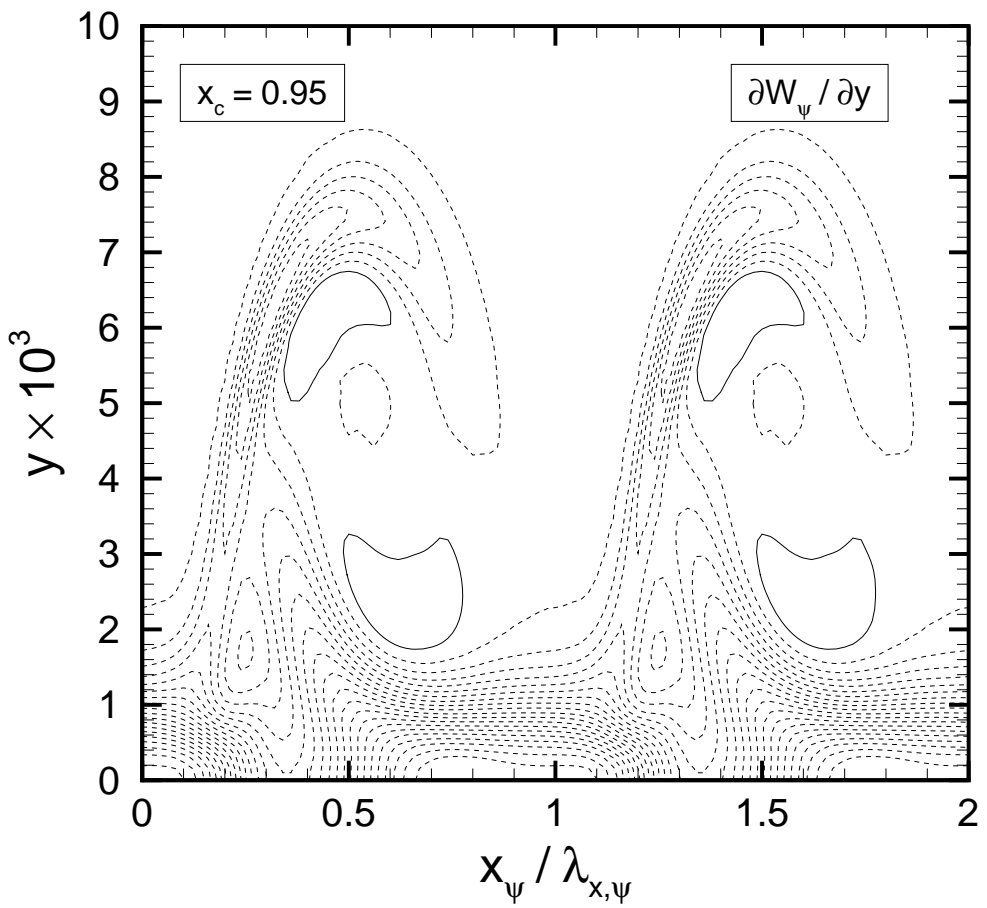

Figure 5.41: Iso-contours of $\partial W_{\psi} / \partial y$ at $x_{c}=0.95$. 


\begin{tabular}{||c|c|c|c|c|c||}
\hline \hline Mode & $b_{\psi}$ (maximal growth) & $\sigma_{r}$ & $\sigma_{i}$ & $f^{*}[\mathrm{~Hz}]$ & $\sigma_{i} / b_{\psi}$ \\
\hline \hline 1 & 1009.2 & 65.18 & 953.6 & 5768 & 0.945 \\
\hline 2 & 639.1 & 31.32 & 599.2 & 3624 & 0.937 \\
\hline 3 & 1480.1 & 14.94 & 1420.3 & 8590 & 0.960 \\
\hline 4 & 773.7 & 12.83 & 726.3 & 4393 & 0.939 \\
\hline 5 & 370.0 & 12.76 & 395.2 & 2390 & 1.068 \\
\hline 6 & 504.6 & 10.97 & 430.3 & 2602 & 0.853 \\
\hline 7 & 235.5 & 7.99 & 175.4 & 1061 & 0.745 \\
\hline 8 & 1614.7 & 7.31 & 1574.2 & 9520 & 0.975 \\
\hline 9 & 269.1 & 7.25 & 228.9 & 1385 & 0.851 \\
\hline 10 & 336.4 & 5.68 & 243.7 & 1474 & 0.724 \\
\hline \hline
\end{tabular}

Table 5.6: Properties of unstable secondary disturbance modes at $x_{c}=0.95$ $\left(N_{s}=16\right)$.

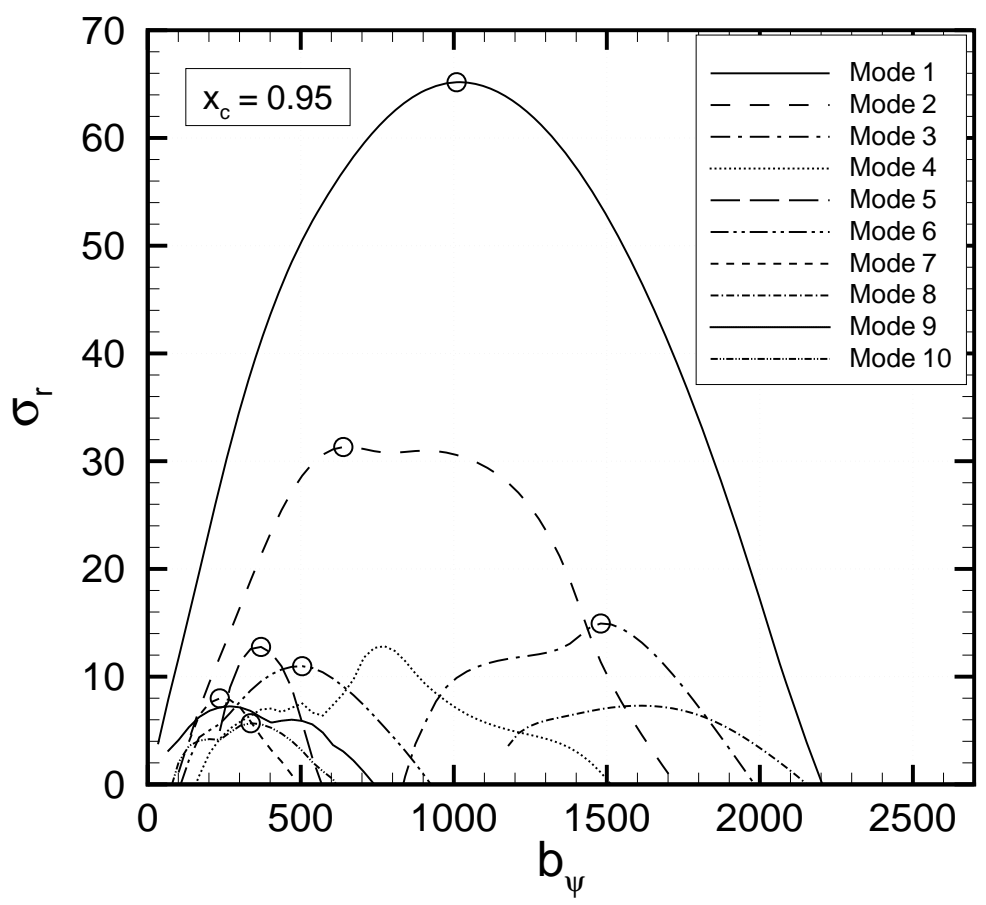

Figure 5.42: Temporal growth rate $\sigma_{r}$ of secondary disturbance modes versus wave number $b_{\psi}$ at $x_{c}=0.95\left(N_{s}=16\right)$. The circles indicate the locations, where the $\left|\bar{w}_{\psi}\right|$-isolines shown in figure $5.43-5.49$ have been extracted. 
mode 2 (see figure 5.44) is located further away from the wall at $y \approx 6 \cdot 10^{-3}$. This mode can be associated with regions of high wallnormal shear $\partial W_{\psi} / \partial y$, even though the secondary velocity fluctuations do not extend to the crest of the primary vortex.

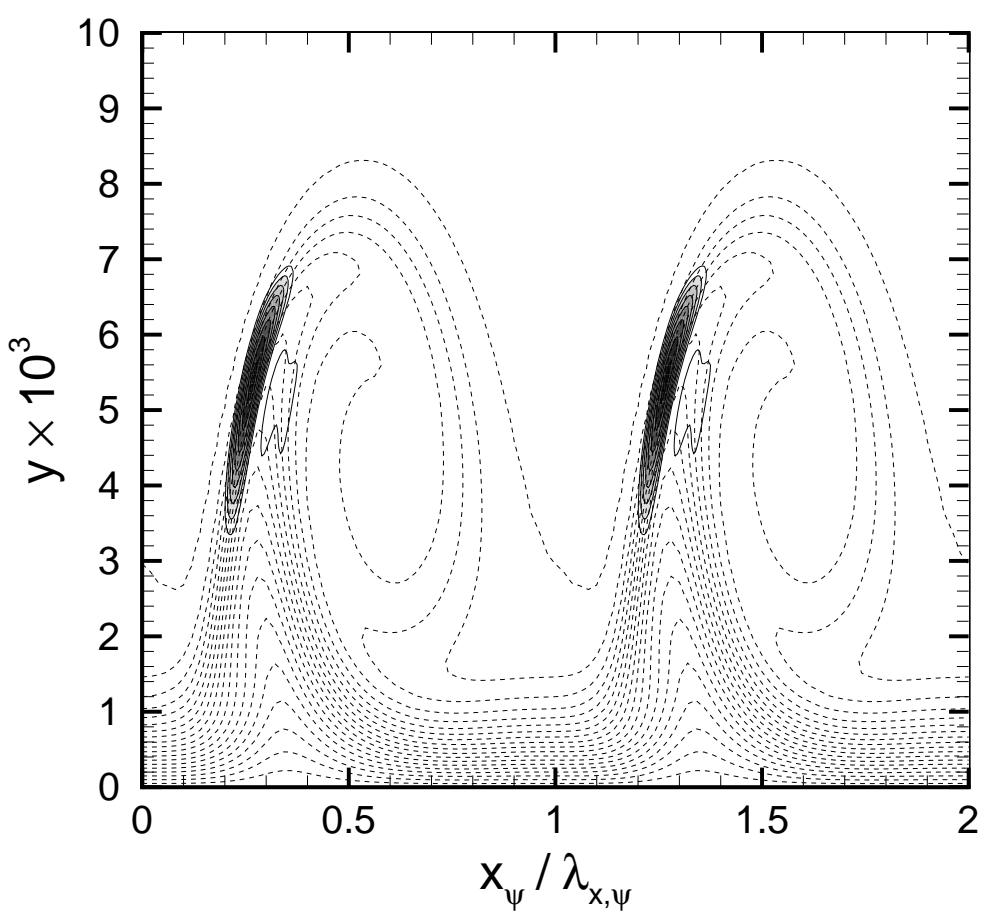

Figure 5.43: $\left|\bar{w}_{\psi}\right|$-isolines of secondary disturbance (mode 1 ) with $b_{\psi}=1009$ at $x_{c}=0.95\left(N_{s}=16\right)$. The dashed lines indicate the $W_{\psi}$-component of the total (primarily disturbed) flow field.

All other secondary disturbance modes exhibit comparatively low temporal growth rates. Mode 3 (see figure 5.45), mode 4, and mode 8 are of "mode II"-type. Mode 6 (see figure 5.47) and mode 7 (see figure 5.48) exhibit high values of $\left|\bar{w}_{\psi}\right|$ both in the region of high wallnormal shear $\partial W_{\psi} / \partial y$ and in the region of low shear between the primary vortices.

While the "mode I"-type disturbances correlate with high positive spanwise shear $\partial W_{\psi} / \partial x_{\psi}$, a secondary disturbance can also be found in the region of high negative spanwise shear in the primarily disturbed flow field. $\left|\bar{w}_{\psi}\right|-$ isolines of this disturbance (mode 5), which has a maximal temporal growth rate $\sigma_{r}=12.8$ and a dimensional frequency $f^{*}=2390 \mathrm{~Hz}$ for $b_{\psi}=370.0$, are shown in figure 5.46. Similarly, the $\left|\bar{w}_{\psi}\right|$-maximum of mode 10 (see figure 5.49) correlates with the region of positive wallnormal shear $\partial W_{\psi} / \partial y$, whereas "mode II"-type disturbances are associated with regions of high negative wallnormal shear near the edge of the boundary layer. 


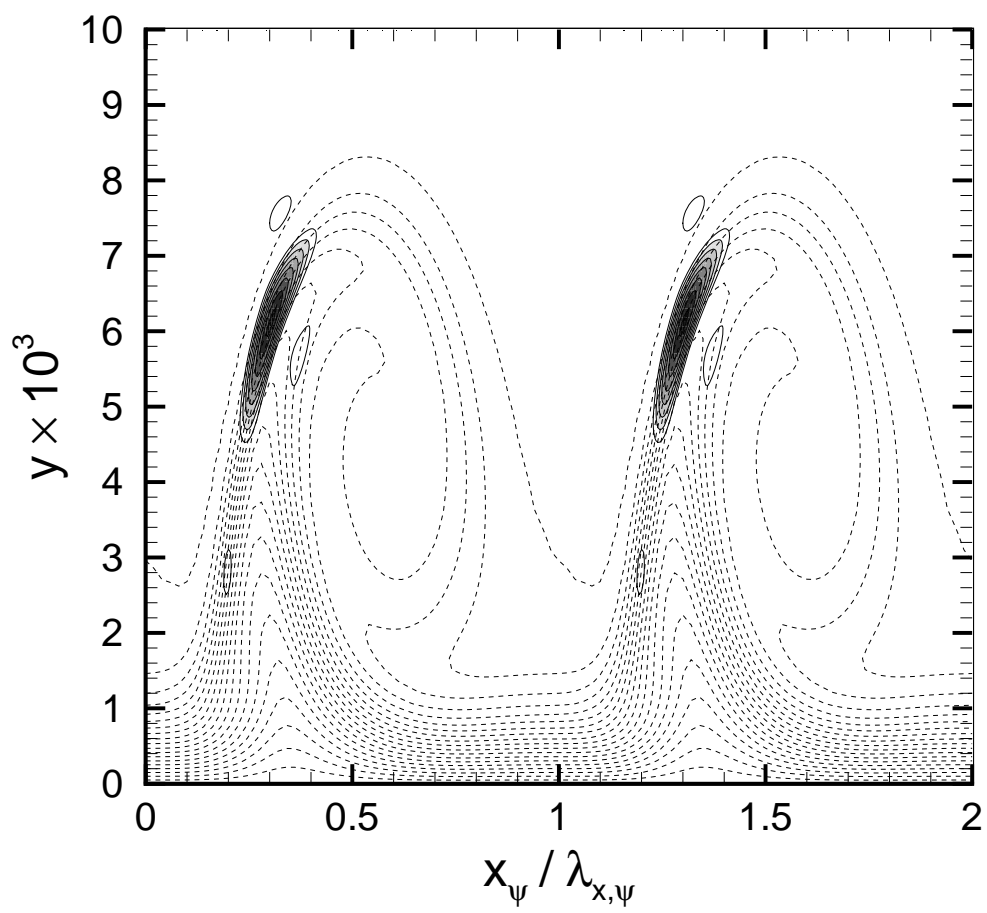

Figure 5.44: $\left|\bar{w}_{\psi}\right|$-isolines of secondary disturbance (mode 2) with $b_{\psi}=639.1$ at $x_{c}=0.95\left(N_{s}=16\right)$. The dashed lines indicate the $W_{\psi}$-component of the total (primarily disturbed) flow field.

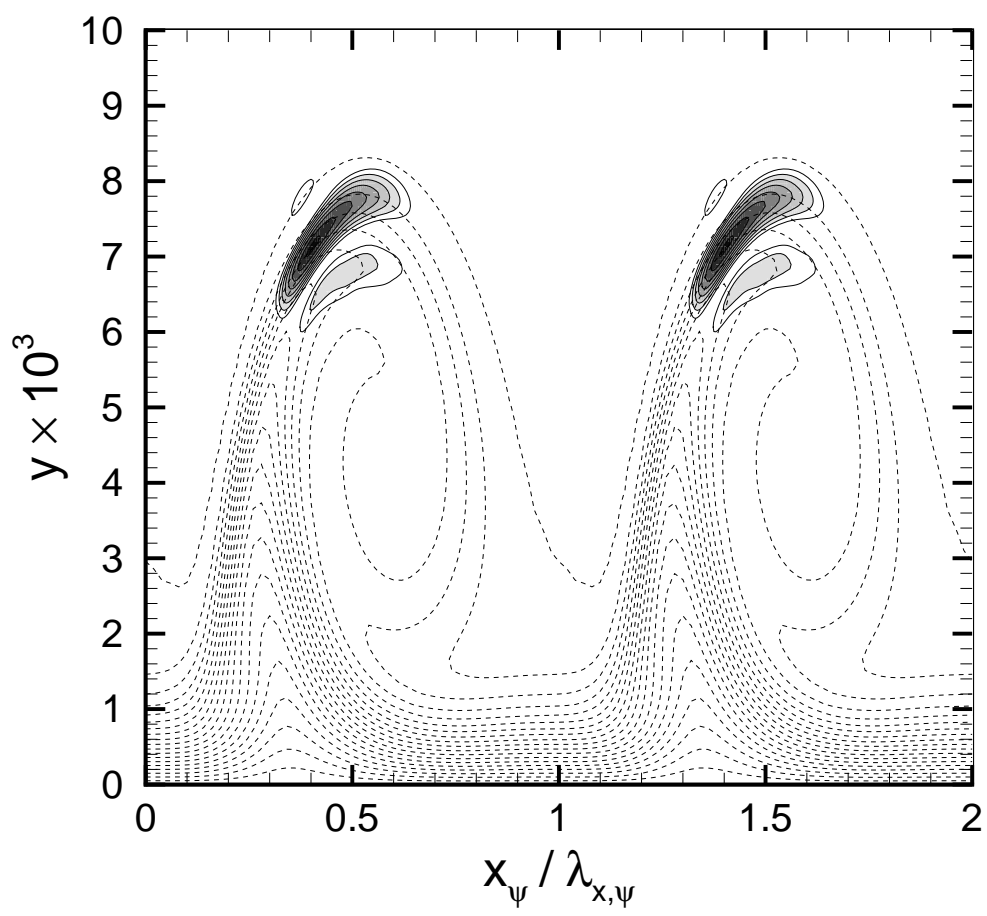

Figure 5.45: $\left|\bar{w}_{\psi}\right|$-isolines of secondary disturbance (mode 3 ) with $b_{\psi}=1480$ at $x_{c}=0.95\left(N_{s}=16\right)$. The dashed lines indicate the $W_{\psi}$-component of the total (primarily disturbed) flow field. 


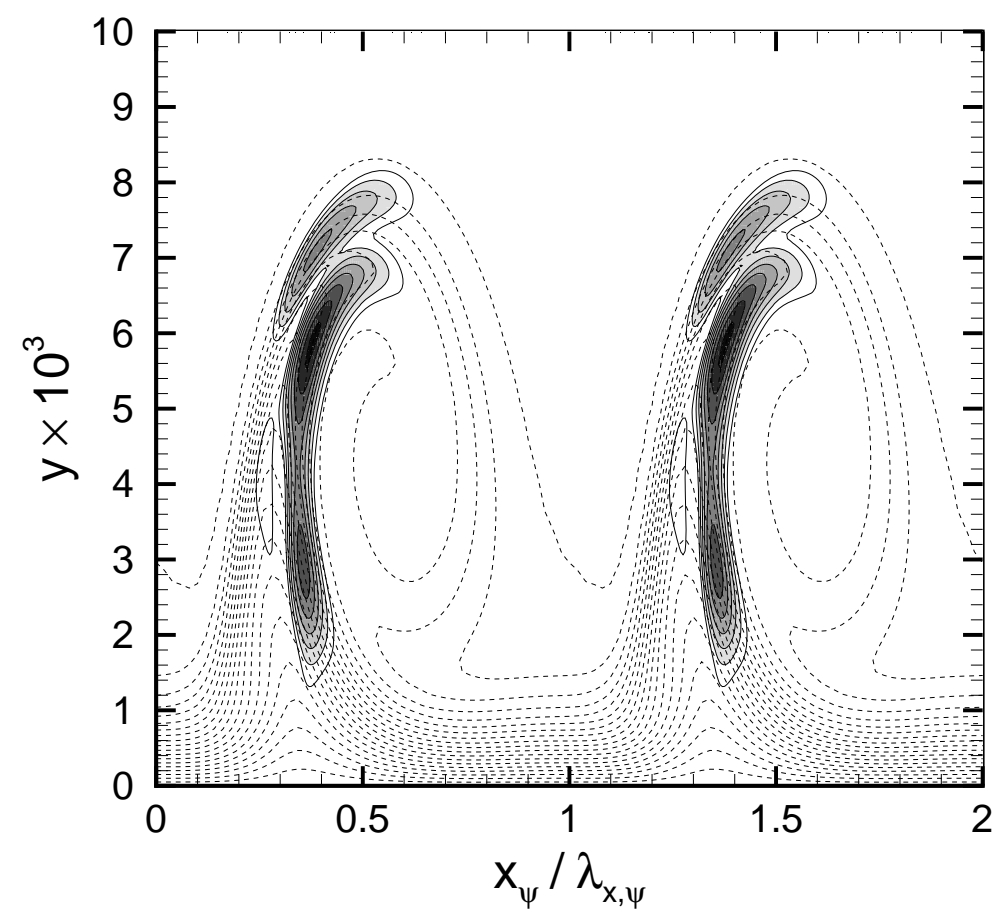

Figure 5.46: $\left|\bar{w}_{\psi}\right|$-isolines of secondary disturbance (mode 5) with $b_{\psi}=370.0$ at $x_{c}=0.95\left(N_{s}=16\right)$. The dashed lines indicate the $W_{\psi}$-component of the total (primarily disturbed) flow field.

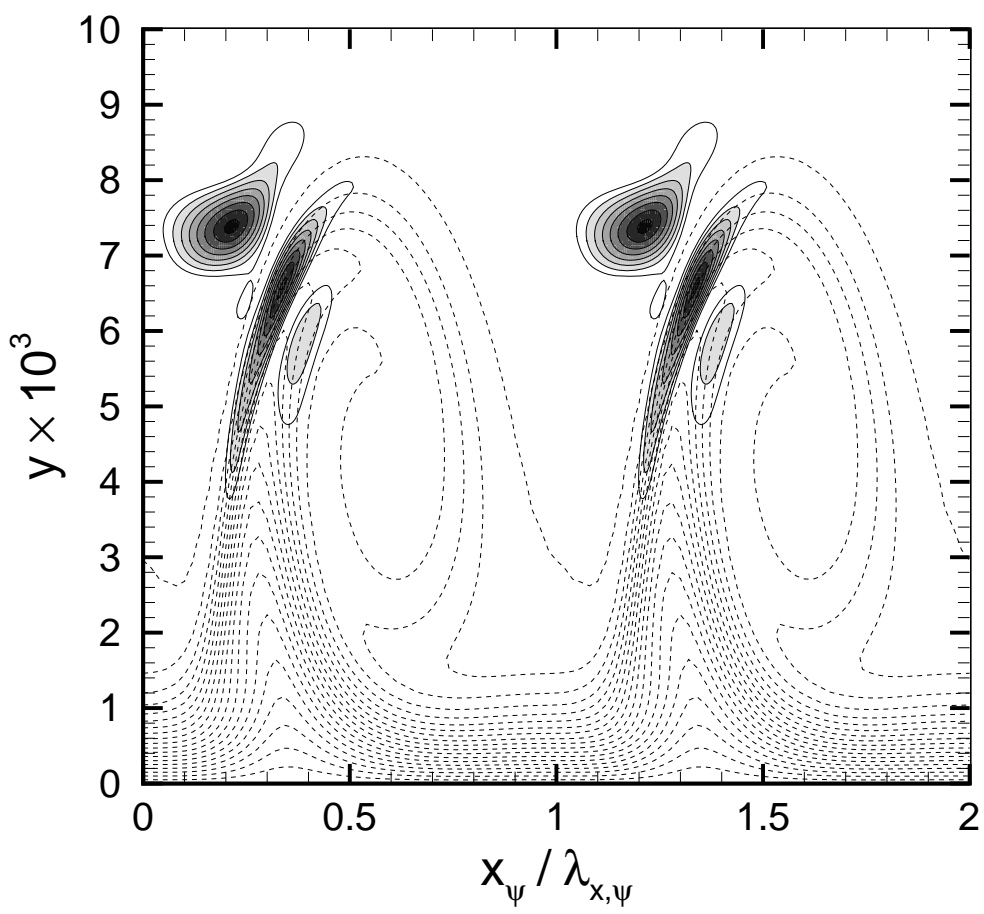

Figure 5.47: $\left|\bar{w}_{\psi}\right|$-isolines of secondary disturbance (mode 6) with $b_{\psi}=504.6$ at $x_{c}=0.95\left(N_{s}=16\right)$. The dashed lines indicate the $W_{\psi}$-component of the total (primarily disturbed) flow field. 


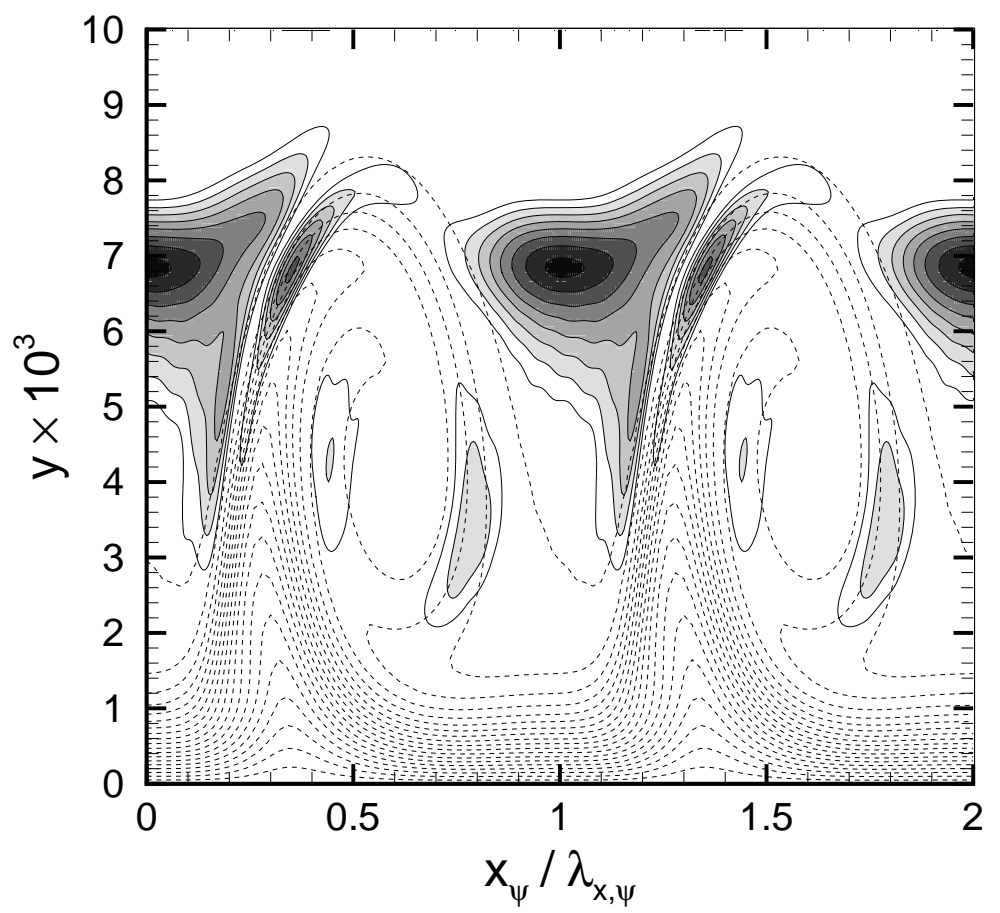

Figure 5.48: $\left|\bar{w}_{\psi}\right|$-isolines of secondary disturbance (mode 7) with $b_{\psi}=235.5$ at $x_{c}=0.95\left(N_{s}=16\right)$. The dashed lines indicate the $W_{\psi}$-component of the total (primarily disturbed) flow field.

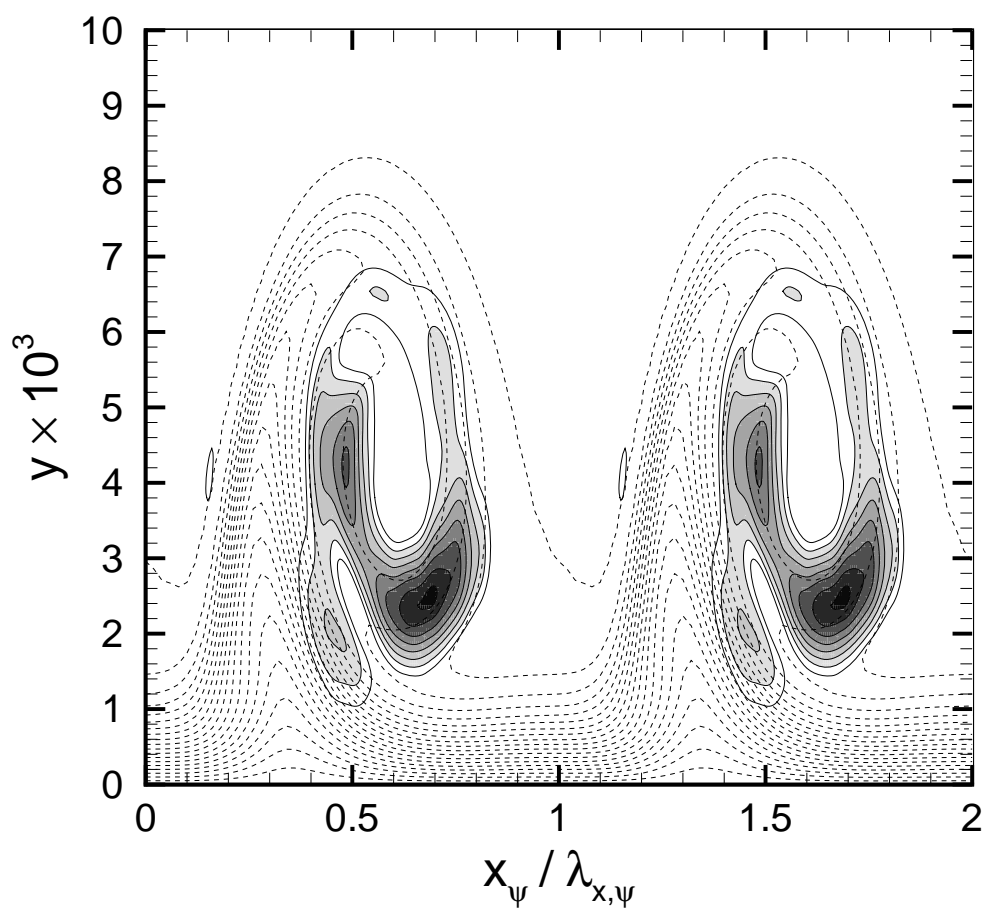

Figure 5.49: $\left|\bar{w}_{\psi}\right|$-isolines of secondary disturbance (mode 10) with $b_{\psi}=336.4$ at $x_{c}=0.95\left(N_{s}=16\right)$. The dashed lines indicate the $W_{\psi}$-component of the total (primarily disturbed) flow field. 


\section{Chapter 6}

\section{Concluding Remarks}

\subsection{Summary}

The three-dimensional, incompressible boundary layer on a swept plate has been analyzed for primary and secondary instability. The attention has been focused on the role of stationary crossflow vortices in the transition process. For that, a model flow has been defined on the basis of the DLR swept plate experiment and corresponding direct numerical simulations. This flow is quasi two-dimensional in the sense, that the (three-dimensional) laminar base flow is constant in spanwise direction. It allows the study of crossflow instability without the influence of surface curvature and compressibility, which significantly simplifies the theoretical treatment.

For the linear nonlocal instability analysis, the parabolized stability equations (PSE) have been used. In a discussion of numerical aspects of these equations, the lower step size limit caused by a residual ellipticity in the PSE has been studied. It has been shown, that neglecting the derivative of the pressure in the $u$-momentum equation removes the lower step size limit for all practical needs. Different ways of generating initial conditions for the PSE have been compared, and the transient behavior of linear PSE results has been studied. If nonparallel initial conditions are specified upstream of the neutral point, only small transients have been observed in the sense, that PSE results for initial conditions specified at different chordwise locations agree well after few steps in downstream direction. Nevertheless, the validity of the PSE assumption is questionable in regions with rapid changes in the base flow. For that reason, PSE results very near to the attachment line have to be interpreted with some caution.

Assuming constant frequency and constant spanwise wave number, the chordwise growth rates of single disturbances have been integrated in order to find those disturbances, which experience the strongest amplification. The results agree well with experimental observations and previous numerical studies. In the nonlinear investigations, a stationary crossflow vortex 
with spanwise wave length $\lambda_{z, c}^{*}=12 \mathrm{~mm}$ and a traveling crossflow wave with the same spanwise wave length and the frequency $f^{*}=135 \mathrm{~Hz}$ have been considered in detail. Both disturbances exhibit very high growth rates according to linear stability theory.

After an outline of central aspects of the nonlinear PSE and a sketch of nonlinear disturbance growth in plane Poiseuille flow, the nonlinear evolution of steady crossflow vortices has been investigated for a variety of different initial amplitudes. A central outcome of the study of disturbance saturation is, that high vortex amplitudes are reached at the end of the plate, if moderate initial amplitudes are specified for the vortex. If the vortex is initialized at high amplitude, it only reaches comparatively low amplitudes. A unique saturation curve for the vortex amplitude does not exist. This phenomenon, which is in agreement with results from previous PSE studies, direct numerical simulations, and solutions obtained by nonlinear critical layer theory, has been investigated further by artificially freezing the laminar base flow. In this approach, saturation of vortex amplitude could only be observed for base flow profiles upstream of $x_{c} \approx 0.3$. Downstream of this point, the saturated solution undergoes a spatial Hopf bifurcation, and stationary solutions with oscillatory behavior in space exist. The wave length of this oscillation is of the order of the chord length of the plate. Similar observations, which emphasize the central role of receptivity in the transition process, have been made for traveling crossflow waves. While a spatial bifurcation could be found as for the stationary vortex, some influence of the (spatially unstable) equilibrium solution on the spatially oscillating state can be observed in the case of the traveling disturbance.

In a study of nonlinear interaction between a steady crossflow vortex and a traveling crossflow wave, the observation, that the presence of traveling waves can significantly lower the amplification of stationary vortices, has been confirmed. However, in spite of their higher growth rates according to linear instability theory, traveling crossflow waves do not strongly affect the evolution of vortices, once the latter disturbances have reached high amplitudes.

Due to the rotation of the vortices, low speed fluid is transported from the near wall region into the outer part of the boundary layer, and fast fluid is moved closer to the wall. This results in regions of high wallnormal and spanwise shear in the outer part of the boundary layer. In addition, the existence of small counter-clockwise rotating vortices very near to the wall has been shown in the flow field distorted by clockwise rotating crossflow vortices.

In a linear temporal secondary instability analysis based on Floquet theory, the secondary instability of the base flow, distorted by steady crossflow vortices, with respect to high-frequency disturbances has been studied for a wide range of primary vortex amplitudes. First, the limit of low primary vortex amplitude has been considered. In this limit, only low-frequency waves, 
which correspond to primary traveling crossflow waves, can be found. These waves are more and more modulated by the primary crossflow vortices, if the amplitude of the vortices increases. It has been demonstrated that, in the frame of Floquet theory, secondary disturbances can only propagate in direction of the primary vortex axis.

First unstable high-frequency disturbances have been found at primary vortex amplitudes around $4 \%$ of the free-stream velocity. At this low primary vortex amplitude, however, the growth rates of high-frequency secondary disturbances are still very low. At $10 \%$ primary vortex amplitude, a large variety of unstable secondary disturbances with moderate temporal growth rates has been found. For each secondary mode, the angular frequency is approximately proportional to the wave number in direction of the primary vortex axis. If the amplitude of the primary vortex is significantly higher than $10 \%$, one high-frequency secondary disturbance by far exceeds all other modes in growth. This disturbance is located on the back of the primary vortex, where both spanwise and wallnormal shear of the distorted base flow exhibit maxima. The structure of this disturbance agrees well with recent measurements by Kawakami et al. [48], who studied the stability characteristics of crossflow vortices with high amplitudes.

\subsection{Outlook}

In the secondary instability analysis presented here, the temporal linear instability of stationary crossflow vortices has been studied. While the formulation discussed in section 5.2 is also valid for traveling primary instability waves, the consideration of both stationary and traveling primary disturbances is more involved. One extension of this work would be to use the secondary disturbance modes as initial conditions for PSE computations. However, the wave numbers and frequencies of strongly amplified secondary disturbances are about one order of magnitude larger than the corresponding parameters of the primary disturbances. Thus, a huge number of modes would be necessary for the PSE computation (cf. equation (4.1)) in order to resolve both primary and secondary disturbances. This leads to enormous memory requirements. On the other hand, such a PSE analysis should not only allow to consider both stationary and traveling primary disturbances simultaneously, it should also capture the experimentally observed nonlinear saturation of secondary disturbances (Bippes [10]). In addition, this extension should directly predict spatial growth rates for the disturbances, which so far are only found by means of an extended Gaster transformation (cf. Koch et al. [53]). 



\section{Bibliography}

[1] C. Airiau and G. Casalis. Nonlinear PSE compared with DNS and experiment. In R. Kobayashi, editor, Proceedings of the IUTAM Symposium on Laminar-Turbulent Transition, pages 85-92. Springer, 1995.

[2] D. Arnal. Boundary layer transition: Predictions based on linear theory. In AGARD Special Course in Transition Modelling, AGARD-R-796, pages 2.1-2.63, 1994.

[3] D. Arnal, E. Coustols, and J.C. Juillen. Experimental and theoretical study of transition phenomena on an infinite swept wing. Rech. Aerosp. 4, 39-54, 1984.

[4] S. Bake, Y.S. Kachanov, and H.H. Fernholz. Subharmonic K-regime of boundary-layer breakdown. In R.A.W.M. Henkes and J.L. van Ingen, editors, Transitional Boundary Layers in Aeronautics, pages 81-88. NorthHolland, 1996.

[5] S. Balachandar, C.L. Streett, and M.R. Malik. Secondary instability in a rotating disk flow. J. Fluid Mech. 242, 323-347, 1992.

[6] F.P. Bertolotti. Linear and nonlinear stability of boundary layers with streamwise varying properties. PhD thesis, The Ohio State University, 1991.

[7] F.P. Bertolotti. DLR Göttingen, 1995. Private communication.

[8] F.P. Bertolotti. On the birth and evolution of disturbances in threedimensional boundary layers. In P.W. Duck and P. Hall, editors, Proceedings of the IUTAM Symposium on Nonlinear Instability and Transition in Three-Dimensional Boundary Layers, pages 247-256. Kluwer, 1996.

[9] H. Bieler. Theoretische Untersuchungen über primäre Instabilitäten in dreidimensionalen Grenzschichtströmungen. DFVLR-FB 86-54, DFVLR, Köln, 1986.

[10] H. Bippes. DLR Göttingen, 1999. Private communication. 
[11] H. Bippes. Basic experiments on transition in three-dimensional boundary layers dominated by crossflow instability. Prog. Aero. Sci. 35, 363412, 1999.

[12] G. Bonfigli and M. Kloker. Three-dimensional boundary-layer transition phenomena investigated by spatial direct numerical simulation. In $\mathrm{H}$. Fasel and W. Saric, editors, Proceedings of the IUTAM Symposium on Laminar-Turbulent Transition. Springer, 1999.

[13] A. Bottaro and B.G.B. Klingmann. On the linear breakdown of Görtler vortices. Eur. J. Mech., B/Fluids 15, 301-330, 1996.

[14] M. Bouthier. Stabilité linéaire des écoulements presque parallèles. J. méchanique 11, 599-621, 1972.

[15] S. Brown and F. Smith. On vortex wave interactions. Part 1. Nonsymmetrical input and cross-flow in boundary layers. J. Fluid Mech. 307, 101-133, 1996.

[16] C.-L. Chang, M.R. Malik, G. Erlebacher, and M.Y. Hussaini. Compressible stability of growing boundary layers using parabolized stability equations. AIAA-Paper 91-1636, 1991.

[17] J.R. Dagenhart, W.S. Saric, M.C. Mousseux, and J.P. Stack. Crossflow vortex instability and transition on a 45-degree swept wing. AIAA-Paper 89-1892, 1989.

[18] H. Deyhle and H. Bippes. Disturbance growth in an unstable threedimensional boundary layer and its dependence on environmental conditions. J. Fluid Mech. 316, 73-113, 1996.

[19] P.G. Drazin and W.H. Reid. Hydrodynamic stability. Cambridge University Press, Cambridge, 1981.

[20] Dubbel. Taschenbuch für den Maschinenbau. Springer, Berlin, 1986.

[21] G. Erlebacher and M.Y. Hussaini. Numerical experiments in supersonic boundary-layer stability. Phys. Fluids A 2, 94-104, 1990.

[22] T.M. Fischer and U. Dallmann. Theoretical investigation of secondary instability of three-dimensional boundary-layer flows. AIAA-Paper 871338, 1987.

[23] T.M. Fischer and U. Dallmann. Primary and secondary stability analysis of a three-dimensional boundary-layer flow. Phys. Fluids A 3, 2378-2391, 1991.

[24] T.M. Fischer, S. Hein, and U. Dallmann. A theoretical approach for describing secondary instability features in three-dimensional boundarylayer flows. AIAA-Paper 93-0080, 1993. 
[25] J.S.B. Gajjar. On the nonlinear evolution of a stationary cross-flow vortex in a fully three-dimensional boundary layer-flow. In P.W. Duck and P. Hall, editors, Proceedings of the IUTAM Symposium on Nonlinear Instability and Transition in Three-Dimensional Boundary Layers, pages 317-326. Kluwer, 1996.

[26] M. Gaster. A note on the relation between temporally increasing and spatially increasing disturbances in hydrodynamic stability. J. Fluid Mech. 14, 222-224, 1962.

[27] M. Gaster. On the effect of boundary layer growth on flow stability. J. Fluid Mech. 66, 465-480, 1974.

[28] W.E. Gray. The effect of wing sweep on laminar flow. Royal Aircraft Establishment, Farnborough, RAE - TM - Aero 255, 1952.

[29] N. Gregory, J.T. Stuart, and W.S. Walker. On the stability of threedimensional boundary layers with application to the flow due to a rotating disk. Phil. Trans. Roy. Soc. A 248, 155-199, 1955.

[30] S. Großmann. Wie entsteht eigentlich Turbulenz. Phys. BI. 51, 641-646, 1995.

[31] H. Haj-Hariri. Characteristics analysis of the parabolized stability equations. Stud. in Appl. Math. 92, 41-53, 1994.

[32] T.S. Haynes and H.L. Reed. Computations in nonlinear saturation of stationary crossflow vortices in a swept wing boundary layer. AIAAPaper 96-0182, 1996.

[33] S. Hein, F.P. Bertolotti, M. Simen, A. Hanifi, and D. Henningson. Linear nonlocal instability analysis - the linear NOLOT code -. DLR-IB 223-94 A56, DLR Institut für Strömungsmechanik, Göttingen, 1994.

[34] S. Hein, T.M. Fischer, and U. Dallmann. Untersuchungen zur sekundären Instabilität einer dreidimensionalen Grenzschichtströmung. DLR-IB 22192 A23, DLR Institut für Theoretische Strömungsmechanik, Göttingen, 1992.

[35] S. Hein, T. Huppertz, and U. Dallmann. Lokale und nichtlokale Instabilitätsanalyse dreidimensionaler Grenzschichtströmungen. Z. angew. Math. Mech. 75, 385-386, 1995.

[36] W. Heisenberg. Über Stabilität und Turbulenz von Flüssigkeitsströmen. Ann. Phys. 74, 577-627, 1924.

[37] H. Helmholtz. Über diskontinuierliche Flüssigkeits-Bewegungen. Monatsberichte der Königlich Preussischen Akademie der Wissenschaften zu Berlin, Berlin, 1868. 
[38] T. Herbert. Secondary instability of boundary layers. Ann. Rev. Fluid Mech. 20, 487-526, 1988.

[39] T. Herbert. Parabolized stability equations. In AGARD Special Course on Progress in Transition Modelling, AGARD-R-793, pages 4.1-4.34, 1994.

[40] T. Herbert. Parabolized stability equations. Ann. Rev. Fluid Mech. 29, 245-283, 1997.

[41] T. Herbert and F.P. Bertolotti. Stability analysis of nonparallel boundary layers. Bull. Amer. Phys. Soc. 32, 2079, 1987.

[42] M. Högberg and D. Henningson. Secondary instability of cross-flow vortices in Falkner-Skan-Cooke boundary layers. J. Fluid Mech. 368, 339357, 1998.

[43] X. Huai, R.D. Joslin, and U. Piomelli. Large-eddy simulation of boundarylayer transition on a swept wedge. J. Fluid Mech. 381, 357-380, 1999.

[44] E. Janke. Nonlinear stability and control of three-dimensional boundary layers. PhD thesis, Old Dominion University, 1998.

[45] E. Janke and P. Balakumar. Nonlinear stability of three-dimensional boundary layers. AIAA-Paper 98-0337, 1998.

[46] R.D. Joslin and C.L. Streett. The role of stationary cross-flow vortices in boundary-layer transition on swept wings. Phys. Fluids 6, 3442-3453, 1994.

[47] Y.S. Kachanov and O.I. Tararykin. The experimental investigation of instability and receptivity of swept wing flow. In D. Arnal and R. Michel, editors, Laminar-Turbulent Transition, IUTAM Symposium, Toulouse, France, 1989, pages 499-509. Springer, 1990.

[48] M. Kawakami, Y. Kohama, and M. Okutsu. Stability characteristics of stationary crossflow vortices in three-dimensional boundary layer. AIAA-Paper 99-0811, 1999.

[49] Lord Kelvin. Hydrokinetic solutions and observations. Phil. Mag. (4) 42, 333-356, 1871.

[50] P.S. Klebanoff, K.D. Tidstrom, and L.M. Sargent. The three-dimensional nature of boundary-layer instability. J. Fluid Mech. 12, 1-39, 1962.

[51] W. Koch. On a degeneracy of temporal secondary instability modes in Blasius boundary-layer flow. J. Fluid Mech. 243, 319-351, 1992.

[52] W. Koch. Nonlinear crossflow saturation in three-dimensional boundary layers. In P.W. Duck and P. Hall, editors, Proceedings of the IUTAM Symposium on Nonlinear Instability and Transition in Three-Dimensional Boundary Layers, Manchester, UK, pages 299-308. Kluwer, 1996. 
[53] W. Koch, F.P. Bertolotti, A. Stolte, and S. Hein. Nonlinear equilibrium solutions in a three-dimensional boundary layer and their secondary instability. Submitted to J. Fluid Mech., 1998.

[54] Y. Kohama, W.S. Saric, and W.S. Hoos. A high-frequency, secondary instability of crossflow vortices, that leads to transition. In Proceedings of the R.A.S. Conference on Boundary-Layer and Control, Cambridge, UK, pages 4.1-4.13, 1991.

[55] T. Lerche. Experimentelle Untersuchung nichtlinearer Strukturbildung im Transitionsprozes̈ einer instabilen dreidimensionalen Grenzschicht. Fortschritt-Bericht VDI Reihe 7, no 310., VDI-Verlag, Düsseldorf, 1997.

[56] F. Li and M.R. Malik. Fundamental and subharmonic secondary instability of Görtler vortices. J. Fluid Mech. 297, 77-100, 1995.

[57] F. Li and M.R. Malik. Mathematical nature of the parabolized stability equations. In R. Kobayashi, editor, Proceedings of the IUTAM Symposium on Laminar-Turbulent Transition, pages 205-212. Springer, 1995.

[58] H.W. Liepmann. Investigation of laminar boundary layer stability and transition on curved boundaries. In NACA Adv. Conf. Rep. No. W-107, 1943.

[59] L.M. Mack. Boundary-layer linear stability theory. In AGARD Special Course on Stability and Transition of Laminar Flow, AGARD-R-709, pages 3.1-3.81, 1984.

[60] M.R. Malik. Numerical simulation of transition in a three-dimensional boundary layer. In F.G. Zhuang and T.L. Zhu, editors, Proceedings of the Tenth International Conference on Numerical Methods in Fluid Dynamics, pages 457-461. Springer, 1986.

[61] M.R. Malik, F. Li, and C.-L. Chang. Crossflow disturbances in threedimensional boundary layers: Nonlinear development, wave interaction and secondary instability. J. Fluid Mech. 268, 1-36, 1994.

[62] M.R. Malik, F. Li, and C.-L. Chang. Nonlinear crossflow disturbances and secondary instabilities in swept-wing boundary layers. In P.W. Duck and P. Hall, editors, Proceedings of the IUTAM Symposium on Nonlinear Instability and Transition in Three-Dimensional Boundary Layers, Manchester, UK, pages 257-266. Kluwer, 1996.

[63] F. Meyer. Numerische Simulation der Transition in dreidimensionalen Grenzschichten. DLR-FB 89-12, DLR, Köln, 1989.

[64] F. Meyer and L. Kleiser. Numerical investigation of transition in 3d boundary layers. In AGARD Conference on Fluid Dynamics of Three-Dimensional Turbulent Shear Flows and Transition, AGARD-R438, pages 16.1-16.17, 1988. 
[65] B. Müller. Experimentelle Untersuchung der Querströmungsinstabilität im linearen und nichtlinearen Bereich des Transitionsgebietes. DLR-FB 90-09, DLR, Köln, 1990.

[66] B. Müller and H. Bippes. Experimental study of instability modes in a three-dimensional boundary layer. In AGARD Conference on Fluid Dynamics of Three-Dimensional Turbulent Shear Flows and Transition, AGARD-R-438, pages 13.1-16.15, 1988.

[67] W. Müller. Numerische Untersuchung räumlicher Umschlagvorgänge in dreidimensionalen Grenzschichtströmungen. PhD thesis, Universität Stuttgart, 1995.

[68] M. Nishioka, M. Asai, and S. lida. An experimental investigation of the secondary instability. In R. Eppler and H. Fasel, editors, IUTAM Symposium on Laminar-Turbulent Transition, pages 37-46. Springer, 1980.

[69] P. Nitschke-Kowsky. Experimentelle Untersuchungen zu Stabilität und Umschlag dreidimensionaler Grenzschichten. DFVLR-FB 86-24, DFVLR, Köln, 1986.

[70] P. Nitschke-Kowsky and H. Bippes. Instability and transition of a threedimensional boundary layer on a swept plate. Phys. Fluids 31, 786-795, 1988.

[71] W.M.F. Orr. The stability or instability of the steady motions of a perfect liquid and of a viscous liquid. Proc. Roy. Irish Acad. 27, 9-68 and 69-138, 1907.

[72] S.A. Orszag. Accurate solution of the Orr-Sommerfeld stability equation. J. Fluid Mech. 50, 689-703, 1971.

[73] P.R. Owen and D.G. Randall. Boundary layer transition on a sweptback wing: a further investigation. Royal Aircraft Establishment, Farnborough, RAE - TM - Aero 330, 1953.

[74] D.S. Park and P. Huerre. Primary and secondary instabilities of the asymptotic suction boundary layer on a curved plate. J. Fluid Mech. 283, 249-272, 1995.

[75] D.I.A. Poll. Some observations of the transition process on the windward face of a long yawed cylinder. J. Fluid Mech. 150, 329-356, 1985.

[76] L. Prandtl. Über Flüssigkeitsbewegung bei sehr kleiner Reibung. In Verhandl. III. Intern. Math. Kongr. Heidelberg, pages 484-491, 1904.

[77] Lord Rayleigh. On the stability, or instability, of certain fluid motions. Proc. London Math. Soc. 11, 57-70, 1880. 
[78] H.L. Reed. Wave interactions in swept-wing flows. AIAA-Paper 841678, 1984. 17th AIAA Fluid Dynamics, Plasma Dynamics, and Laser Conference, Snowmass, Colorado.

[79] H.L. Reed. Wave interactions in swept-wing flows. Phys. Fluids 30, 34193426, 1988.

[80] H.L. Reed and W.S. Saric. Stability of three-dimensional boundary layers. Ann. Rev. Fluid Mech. 21, 235-284, 1989.

[81] O. Reynolds. An experimental investigation of the circumstances which determine whether the motion of water shall be direct or sinuous, and of the law of resistance in parallel channels. Phil. Tran. Roy. Soc. London A 186, 123-164, 1883.

[82] W. Saric and L.G. Yeates. Experiments on the stability of crossflow vortices in swept wing flows. AIAA-Paper 85-0493, 1985.

[83] H. Schlichting. Zur Entstehung der Turbulenz bei der Plattenströmung. Nachr. Ges. Wiss. Göttingen, Math. Phys. Klasse pages 182-174, 1933.

[84] H. Schlichting and K. Gersten. Grenzschicht-Theorie. Springer, Berlin, 1997.

[85] G. Schrauf, Th. Herbert, and G. Stuckert. Evaluation of transition in flight tests using nonlinear PSE analysis. AIAA-Paper 95-1801, 1995.

[86] G.B. Schubauer and H. Skramstad. Laminar boundary-layer oscillations and transition on a flat plate. J. Res. Nat. Bur. Stand. 38, 251-292, 1947.

[87] M. Simen. Lokale und nichtlokale Instabilität hypersonischer Grenzschichtströmungen. DLR-FB 93-31, DLR, Köln, 1993.

[88] M. Simen, F.P. Bertolotti, S. Hein, A. Hanifi, D. Henningson, and U. Dallmann. Nonlocal and nonlinear instability theory. In S. Wagner, J. Périaux, and E.H. Hirschel, editors, Computational Fluid Dynamics 1994, Proc. ECCOMAS Conference, Stuttgart 1994. John Wiley \& Sons, 1994.

[89] F. Smith, S. Brown, and P. Brown. Initiation of three-dimensional nonlinear transition paths from an inflectional profile. Eur. J. Mech., B/Fluids 12, 447-473, 1993.

[90] A. Sommerfeld. Ein Beitrag zur hydrodynamischen Erklärung der turbulenten Flüssigkeitsbewegungen. In Proceedings 4th International Congress of Mathematicians, Rome, vol. III, pages 116-124, 1908. 
[91] A. Stolte, F.P. Bertolotti, S. Hein, M. Simen, and U. Dallmann. Nichtlokale und nichtlineare Instabilitätsuntersuchungen an kompressiblen Strömungen. DLR-IB 223-95 A54, DLR Institut für Strömungsmechanik, Göttingen, 1995.

[92] S. Takagi and N. Itoh. Observation of travelling waves in the threedimensional boundary-layer along a yawed cylinder. Fluid Dyn. Res. 14, 167-189, 1994.

[93] W. Tollmien. Über die Entstehung der Turbulenz. Nachr. Ges. Wiss. Göttingen, Math. Phys. Klasse pages 21-44, 1929.

[94] M. Wagner. Numerische Untersuchungen zum laminar-turbulenten Übergang in zwei- und dreidimensionalen Grenzschichten. DLR-FB 9236, DLR, Köln, 1992.

[95] M. Wang, Th. Herbert, and G.K. Stuckert. Crossflow-induced transition in compressible swept-wing flows. AIAA-Paper 94-2374, 1994. 25th AIAA Fluid Dynamics Conference, Colorado Springs, Colorado.

[96] M. Wiegel. Experimentelle Untersuchung von kontrolliert angeregten dreidimensionalen Wellen in einer Blasius-Grenzschicht. FortschrittBericht VDI Reihe 7, no 312, VDI-Verlag, Düsseldorf, 1997.

[97] T. Wintergerste and L. Kleiser. Breakdown of a crossflow vortex in a three-dimensional boundary layer. In J.-P. Chollet et al., editor, Direct and Large-Eddy Simulation II, pages 179-190. Kluwer, 1997.

[98] R. Zurmühl. Matrizen und ihre technischen Anwendungen. Springer, 3rd edition, 1961. 


\section{Acknowledgements}

The present work would not have been possible without the guidance and support of mentors, colleagues, and friends. I am especially indebted to my committee chair Professor Dr. H. Eckelmann, who provided me with a sound foundation in fluid mechanics, pointed me to the German Aerospace Center (DLR), and continuously accompanied and supported my work in numerous ways. I also thank my co-chair Professor Dr. D. Ronneberger for his support and for reviewing this dissertation.

I am further indebted to my advisor Priv.Doz. Dr. U. Dallmann for the chance to perform this work in the department of Transition and Turbulence at DLR Göttingen, for his efforts in getting me on the right tracks, for fruitful discussions and his interest in this work.

I am truly thankful to Dr. F.P. Bertolotti and to Dr. W. Koch for their profound guidance and for their unrestricted support, patience and encouragement. They have contributed many very stimulating and helpful ideas. Furthermore, Dr. F.P. Bertolotti provided me with his PSE codes and supported me in using and extending them. Dr. W. Koch provided me with the tools for secondary instability analysis and guided me to apply them to answer the right questions. He carefully revised the manuscript of this dissertation and gave many helpful and constructive comments.

Many thanks to all my colleagues, especially to Dr. H. Bippes, Dipl.Ing. S. Hein, Dr. E. Janke, Dr. T. Lerche, and Dipl.-Phys. A. Poethke, for many helpful discussions, for all their support, and for the pleasant atmosphere at work. The effords of Dipl.-Ing. G. Bonfigli and Dr. M. Kloker from Universität Stuttgart for providing the DNS results included in figure 4.23 are gratefully acknowledged.

I am grateful to Dipl.-Phys. M. Klein for his support regarding the use of workstations and software, and to D. Welke for her support in all organizational matters.

The support of the "Graduiertenkolleg für Strömungsinstabilitäten und Turbulenz" and of the Deutsche Forschungsgemeinschaft under the contract Ko $1722 / 2-1$ is gratefully acknowledged.

Last, but not least, a special word of appreciation goes to my wife Petra for her understanding, support and encouragement. 



\title{
Vita
}

\section{Personal Data}

$\begin{array}{ll}\text { Name } & \text { Andreas Stolte } \\ \text { Date of birth } & \text { 30 September } 1969 \\ \text { Birthplace } & \text { Harderberg } \\ \text { Family status } & \text { married } \\ \text { Nationality } & \text { German }\end{array}$

\section{Academic Education}

\author{
$05 / 89$ \\ Abitur, Gymnasium Carolinum, Osnabrück \\ 10/90 - 07/92 Studies of Physics and Mathematics, \\ Albert-Ludwigs-Universität Freiburg \\ 07/92 Vordiplom in Physics und Mathematics \\ 08/92 - 09/93 University of Glasgow, Scotland \\ 04/93 - 06/93 Theory-project with Prof. Laing, Glasgow \\ Propagation of Alfvén waves in non-uniform magnetic fields \\ 06/93 - 09/93 Summer-project with Prof. Whitehead, Glasgow \\ Symmetries and decomposition of dendritic trees \\ since 10/93 Georg-August-Universität, Göttingen \\ $11 / 95$ \\ Diplom in Physics \\ Nichtlineare, lokale und nichtlokale Stabilitätsuntersuchungen \\ an kompressiblen Strömungen
}

Göttingen, 20 September 1999 
